\title{
Understanding inter-tissue crosstalk during zebrafish cardiovascular development
}

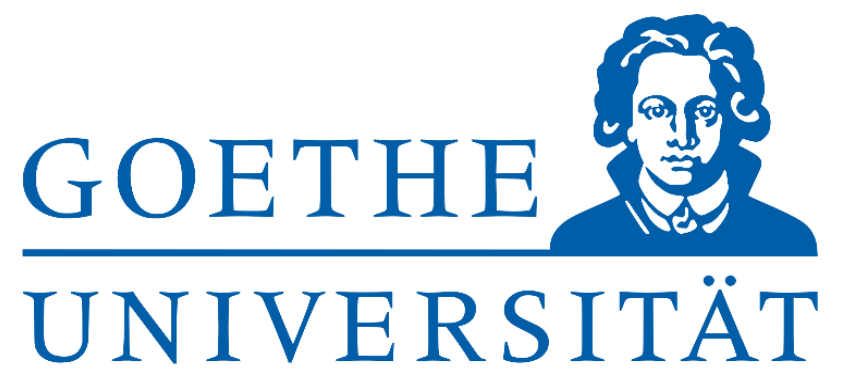

FRANKFURT AM MAIN

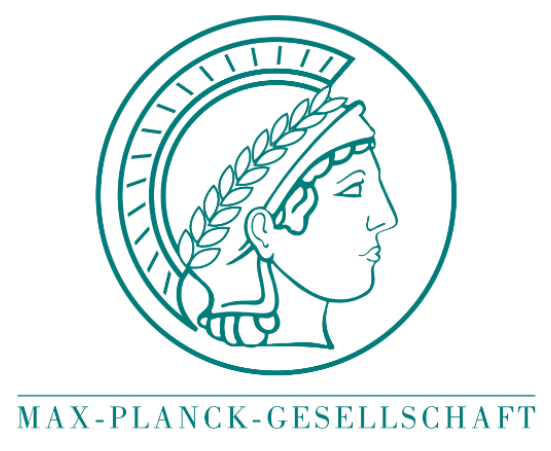

\author{
Dissertation \\ zur Erlangung des Doktorgrades \\ der Naturwissenschaften \\ vorgelegt beim Fachbereich 15 \\ der Johann Wolfgang Goethe-Universität \\ in Frankfurt am Main
}

von

Giulia L. M. Boezio

aus Milano, Italien

Frankfurt 2021

(D30) 

vom Fachbereich Biowissenschaften (FB15) der Johann

Wolfgang Goethe - Universität als Dissertation angenommen.

Dekan: $\quad$ Prof. Dr. Sven Klimpel

Gutachter: $\quad$ Prof. Dr. Didier Y. R. Stainier

Prof. Dr. Amparo Acker-Palmer

Datum der Disputation: 27.09.2021 



\section{REVIEWERS}

Prof. Didier Y. R. Stainier, Ph.D.

Department of Developmental Genetics

Max Planck Institute for Heart and Lung Research

Bad Nauheim, Germany

and

Prof. Amparo Acker-Palmer, $\mathrm{PhD}$

Department of Molecular and Cellular Neurobiology

Institute of Cell Biology and Neuroscience

Johann Wolfgang Goethe University

Frankfurt am Main, Germany 

Between the fifth and tenth days, the lump of stem cells differentiates into the overall building plan of the embryo and its organs. It is like a lump of iron turning into a space shuttle. In fact, it is the profoundest wonder we can imagine and accept, and at the same time so usual that we have to force ourselves to wonder about the wondrousness of this wonder.

Miroslav Holub (1990)

La scienza richiede tutta la vita dai suoi seguaci, non tollera sorte, né temporanei abbandoni, né tiepidi zeli, né avidità di lucro e domanda, anche prima del genio, pazienza, pazienza, pazienza.

Science requires the entire life from its followers, it tolerates neither fate, nor temporary quitting, nor halfhearted enthusiasm, nor greed for profit, and it demands, even before the genius, patience, patience, patience.

Angelo Mosso 



\section{Table of Contents}

I Abbreviations 1

1 Introduction 3

1.1 The cardiovascular system and its evolution in vertebrates 3

1.2 Embryonic development of the heart and greater vessels 4

1.3 Cell-types forming the heart and their origin $\quad 6$

1.4 Communication between different cell types 9

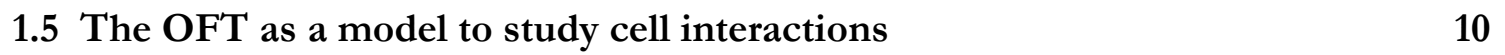

1.5.1 TGF $\beta$ signaling and its various roles during embryonic development ...................... 11

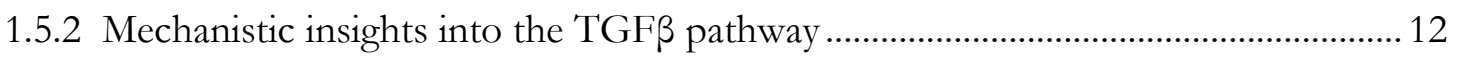

1.5.3 The TGF $\beta$ pathway in cardiovascular development.................................................... 14

1.5.4 The role Alk5/Tgfbr1 in heart development and aortic aneurysm formation .........16

1.6 Another hub of interactions: the epicardium-myocardium surface 17

1.6.1 The role of the epicardium during development and regeneration ............................. 17

1.6.2 The epicardium-myocardium crosstalk during development ....................................... 19

1.6.2.1 Molecular factors implicated in the epicardium-myocardium crosstalk 20

2 Aims of the study $\quad 22$

3 Materials and methods $\quad 25$

3.1 Materials $\quad 25$

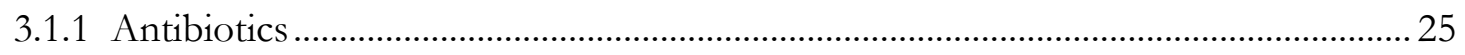

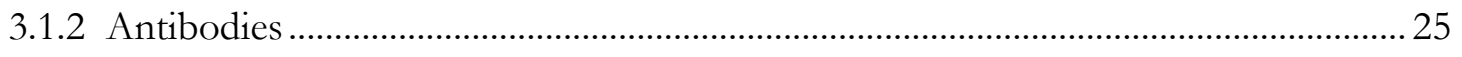

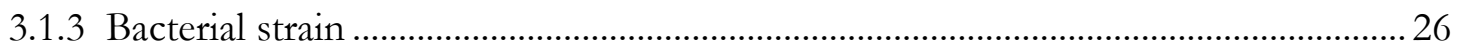

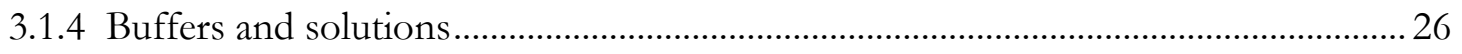

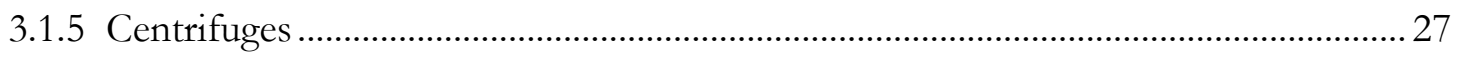

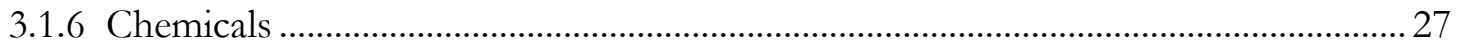

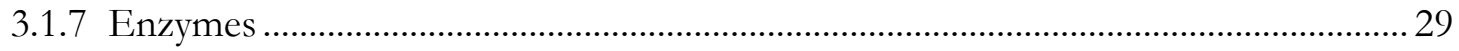

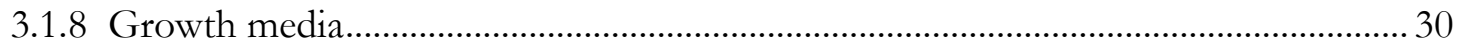

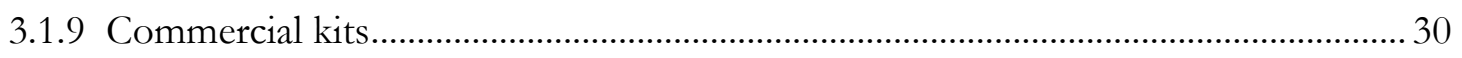

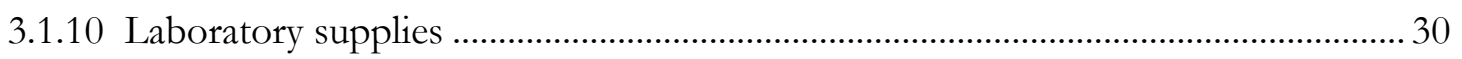

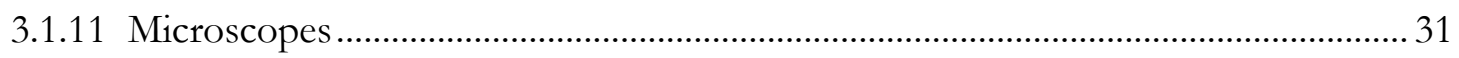

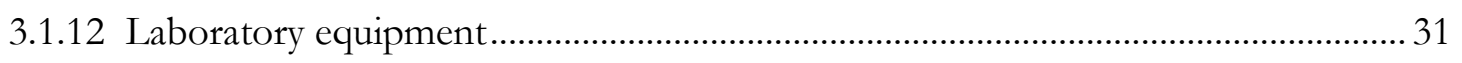

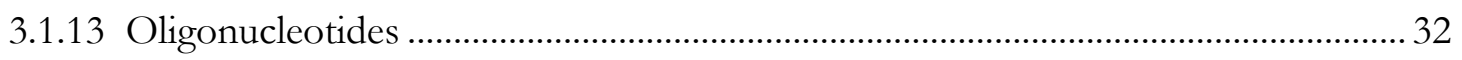

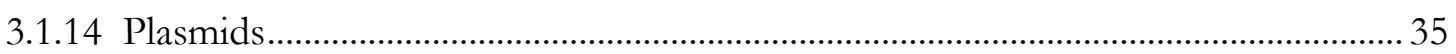




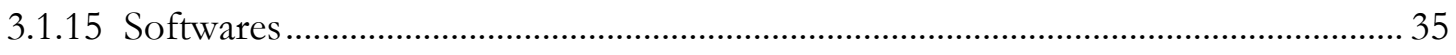

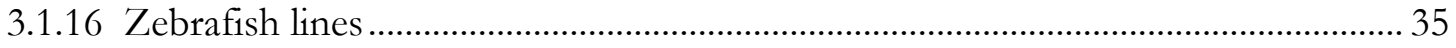

$\begin{array}{ll}3.2 \text { Methods } & 37\end{array}$

3.2.1 Zebrafish maintenance and breeding............................................................................ 37

3.2.2 RNA and DNA microinjections into once-cell stage zebrafish embryos.................. 37

3.2.3 DNA and RNA isolation from zebrafish embryos....................................................... 38

3.2.4 Embryonic heart isolation and RNA extraction........................................................... 38

3.2.5 cDNA synthesis and real-time quantitative PCR (RT-qPCR) .................................... 38

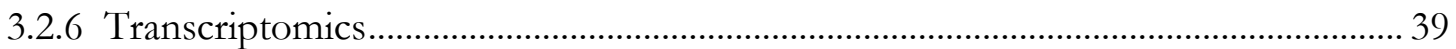

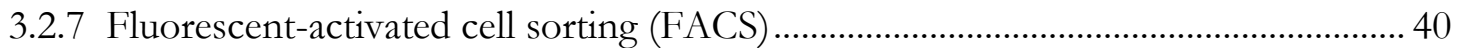

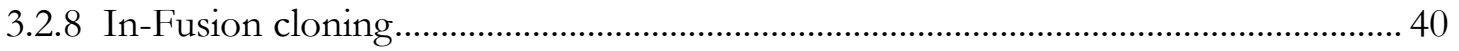

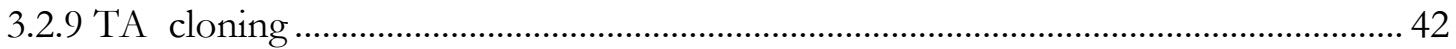

3.2.10 E. coli competent cell transformation ........................................................................... 42

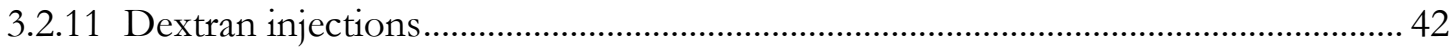

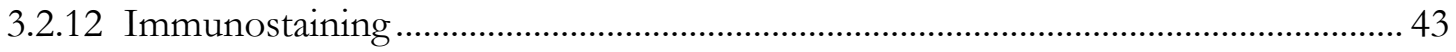

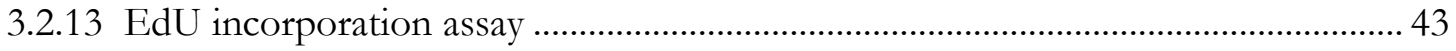

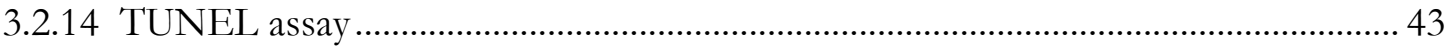

3.2.15 Whole mount in situ hybridization...................................................................................... 44

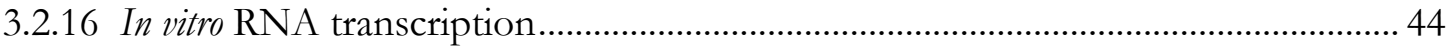

3.2.17 CRISPR/Cas9-mediated mutagenesis ........................................................................... 45

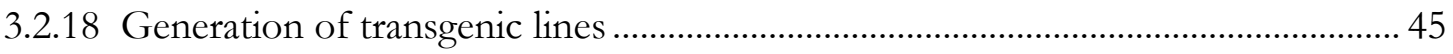

3.2.19 Genotyping High-Resolution Melt (HRM) analysis ..................................................... 46

3.2.20 Transmission electron microscopy (TEM) ..................................................................... 46

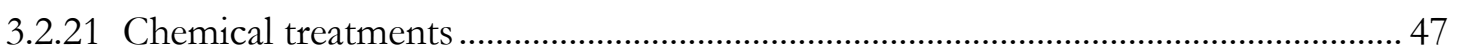

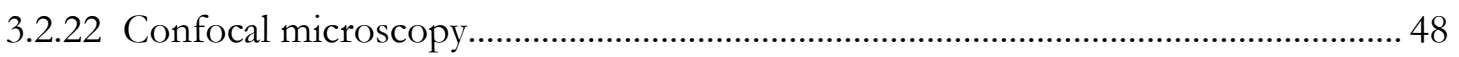

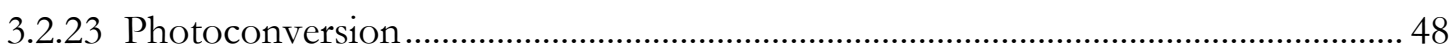

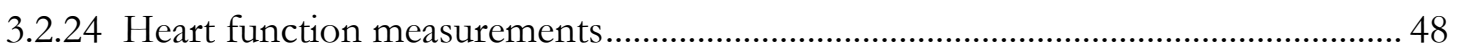

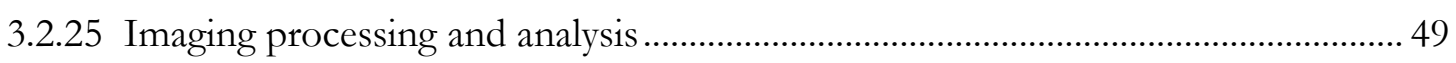

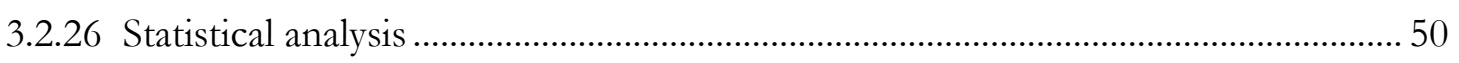

4 Results 51

4.1 The endothelium-smooth muscle crosstalk in the developing zebrafish cardiac outflow tract is orchestrated by TGF- $\beta$ /Alk5 signaling 51

4.1.1 The TGF- $\beta$ pathway is active in the developing heart and enriched in the forming OFT

4.1.2 Mutating alk5 leads to specific defects in OFT size and expansion............................ 56

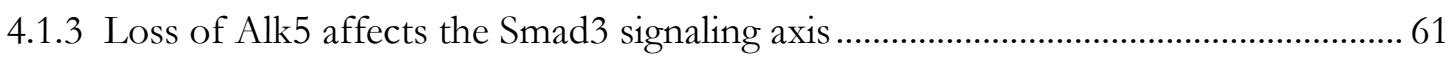


4.1.4 Alk5 inhibitor recapitulates the morphological phenotype of alk.5 mutants. .63

4.1.5 Alk5 limits OFT EC proliferation from early developmental stages .65

4.1.6 Alk5 promotes the formation of the ventral artery and EC displacement from the OFT .67

4.1.7 SMC proliferation and organization are regulated by Alk 5 to promote the formation of the OFT wall. .68

4.1.8 Alk5 regulates the extracellular environment in the cardiac outflow tract ................ 72

4.1.9 Alk5 is required in endothelial cells to promote OFT formation and function ....... 74

4.1.10 Alk5 signaling regulates ECM gene expression to orchestrate OFT development

4.1.11 The OFT-enriched ECM component gene fbln 5 acts downstream of Alk5 to regulate EC proliferation and ECM organization.

4.2 The epicardium-myocardium crosstalk is essential for cardiomyocyte growth during zebrafish cardiac development

4.2.1 Generation of independent models of impaired epicardial coverage in zebrafish larvae.

4.2.2 Lack of epicardial coverage leads to impaired ventricular growth without affecting cardiomyocyte number.

4.2.3 Lack of epicardial coverage leads to impaired ventricular size increase. .95

4.2.4 Identification of a temporal window crucial for the role of the epicardium in cardiac development.

4.2.5 The presence of the epicardial mechanical barrier is not sufficient to support cardiac growth

4.2.6 The epicardium serves as a reservoir of signaling molecules necessary for myocardial development

4.2.7 Vegf and Fgf signaling are crucial in the epicardium-myocardium crosstalk during zebrafish heart development.

\section{Discussion}

5.1 The endothelium-smooth muscle crosstalk in the developing cardiac outflow tract is orchestrated by TGF- $\beta$ /Alk 5 signaling

5.1.1 The development of the OFT, a hub for intercellular interactions.

5.1.2 The role of Alk5/ TGF- $\beta$ in the OFT is cell-type- and time-dependent

5.1.3 ECs regulate SMC behavior by modulating the ECM environment.

5.1.4 Revisiting the role of TGF- $\beta$ in aortic diseases

\subsection{The epicardium-myocardium crosstalk is essential for cardiomyocyte volume} increase during zebrafish cardiac development

5.2.1 Generation of three different models of epicardial impairment: similarities and differences. 
5.2.2 The role of the epicardium in regulating $\mathrm{CM}$ volume increase and proliferation .117

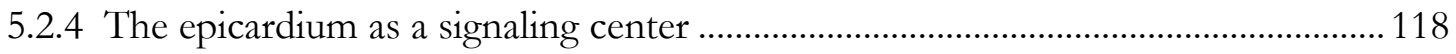

6 Conclusions 121

$\begin{array}{lll}7 & \text { Summaries } & 123\end{array}$

7.1 Zusammenfassung 123

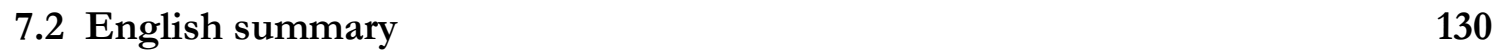

8 Bibliography 136

$\begin{array}{ll}\text { Acknowledgments } & 153\end{array}$

$\begin{array}{ll}\text { Curriculum Vitae } & 157\end{array}$ 


\section{Abbreviations}

\begin{tabular}{|c|c|}
\hline Abbreviation & Description \\
\hline hpf & Hours post-fertilization \\
\hline dpf & Days post-fertilization \\
\hline OFT & Outflow tract \\
\hline $\mathrm{VA}$ & Ventral artery/ ventral aorta \\
\hline $\mathrm{EC}$ & Endothelial cell \\
\hline SMC & Smooth muscle cell \\
\hline $\mathrm{ECM}$ & Extracellular matrix \\
\hline Alk5 & Activin-like kinase domain receptor 5 \\
\hline$f b \ln 5$ & Fibulin-5 \\
\hline TGF- $\beta$ & Transforming growth factor $-\beta$ \\
\hline FGF & Fibroblast growth factor \\
\hline VEGF & Vascular endothelial growth factor \\
\hline $\mathrm{CM}$ & Cardiomyocyte \\
\hline EMT & Epithelial-to-mesenchymal transition \\
\hline $\mathrm{PEO}$ & Proepicardial organ \\
\hline EPDC & Epicardial derived cell \\
\hline $\mathrm{BAC}$ & Bacterial artificial chromosome \\
\hline EGFP & Enhanced green fluorescent protein \\
\hline nls & Nuclear localization signal \\
\hline
\end{tabular}


Introduction 


\section{Introduction}

\subsection{The cardiovascular system and its evolution in vertebrates}

The cardiovascular system is essential to transport oxygen $\left(\mathrm{O}_{2}\right)$ and nutrients to the entire body and eliminate carbon dioxide and waste materials. It is one of the first systems to form in the embryo and is required for the viability of the animal (Baldwin, 1996).

In all vertebrates, the cardiovascular system is composed of a heart, functioning as a pump for blood flow, and different kinds of blood vessels which are responsible to deliver $\mathrm{O}_{2}$ and nutrients to the peripheral tissues of the organism. Blood vessels can be divided into arteries and veins, with the former carrying blood away from the heart and the latter bringing it back (Baldwin, 1996).

In different species of vertebrates, the heart can be composed of 2,3 , or 4 chambers, according to the presence of lungs and the need to divide the pulmonary circulation from the systemic one (Moorman and Christoffels, 2003; Simoes-Costa et al., 2005; Jensen et al., 2013).

Fish which lack proper lungs have a single circulatory system and the blood becomes oxygenated in the gills as soon as it exits the heart and prior to delivery to the body. Therefore, the heart is composed of only two chambers: one atrium, which receives the blood, and one ventricle, which pumps it out (Simoes-Costa et al., 2005; Jensen et al., 2013). In addition, fish develop a subsidiary chamber called bulbus arteriosus (BA), the orthologue of the mammalian outflow tract (OFT) and the root of the great arteries, that serves to buffer the high blood pressure deriving from the ventricle (Grimes and Kirby, 2009; Grimes et al., 2010).

Amphibians and most reptiles have a three-chambered heart (two atria and one ventricle), while mammals and birds need a more efficient way to distribute oxygenated blood through the organism due to their metabolic rates. Therefore, they develop a dual circulatory system and a four-chambered heart, which pumps oxygenated blood to the body and non-oxygenated blood to the lungs (Moorman and Christoffels, 2003; Simoes-Costa et al., 2005; Jensen et al., 2013).

Multiple model organisms have been used to study the development of the cardiovascular system, each with its strengths and weaknesses. Zebrafish has been recognized to be one of the optimal models for these studies. Its advantages rely mainly on the transparency of its embryos, which allows for live imaging and recording of heart function during developmental stages, its ability to survive for several days without a functional cardiovascular system, and its resemblance to the human heart in terms of cellular and molecular processes (Stainier and Fishman, 1994; Staudt and Stainier, 2012; D. R. Brown et al., 2016). For the purpose of this study, I will only focus on the development of mouse and zebrafish hearts. 


\subsection{Embryonic development of the heart and greater vessels}

During the initial phases of embryonic development, the heart forms and starts to function. In human, the heart starts beating at the end of week 4, while in mouse, the heartbeat is established at E9.0 (and started around E8.0) (Tyser et al., 2016). In zebrafish, due to its fast development, the heart starts contracting at 22-24 hours post fertilization (hpf) (Kimmel et al., 1995).

A
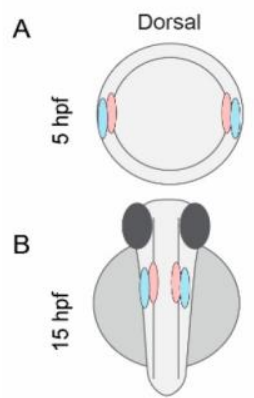

C

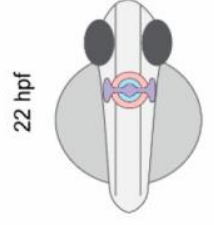

D

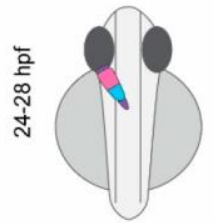

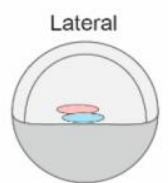
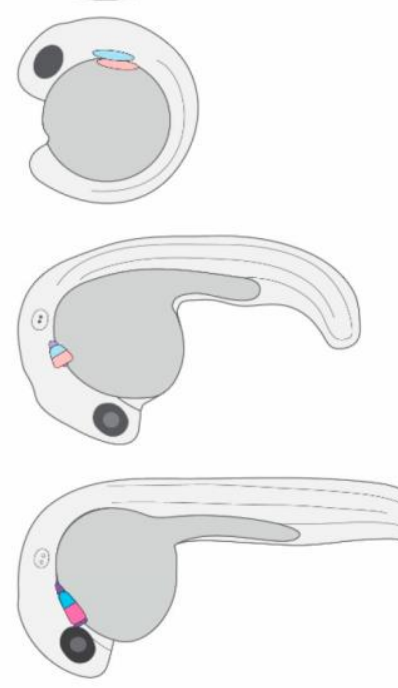

$\mathrm{E}$

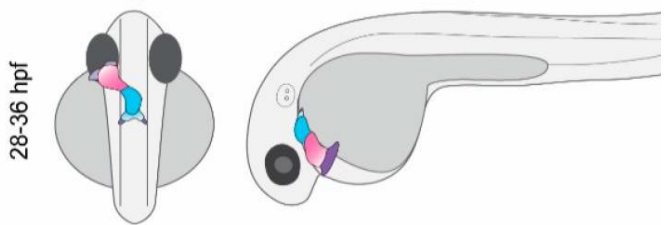

$\mathrm{F}$

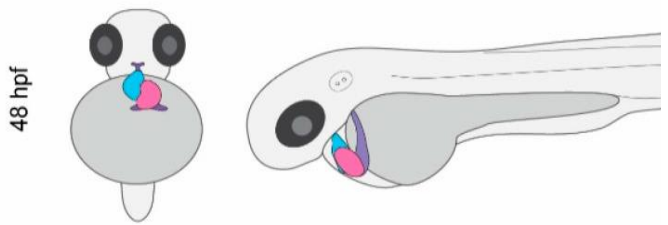

G

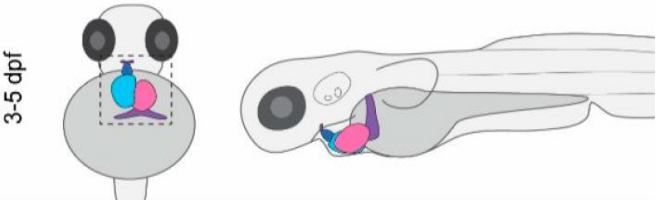

Figure 1: Schematic representation of zebrafish heart development.

A) Blastula stage; atrial and ventricular precursors arise in the median zone, B) Somitogenesis; cardiac precursors form the lateral plate mesoderm; C) Precursors migrate to the midline where they give rise to the cardiac disc; D) Between 24 and 28 hpf, formation of a linear heart tube. E-F) During late embryonic stages, the heart tube jogs and loops and positions the ventricle on the right and the atrium on the left. G) Larval stages; the heart grows and other morphogenetic processes take place (proliferation, trabeculation, valve formation...). Light blue, ventricle; magenta, atrium; blue, bulbus arteriosus (G); purple, arterial, and venous poles. Image adapted from (D. R. Brown et al., 2016). License: CC BY 2.0.

The development of the heart begins with the migration of the cardiac progenitors to the midline of the embryo. Cardiac progenitors are already specified and are segregated into atrial and ventricular compartments by gastrulation stages (Keegan et al., 2004; Ivanovitch et al., 2020) (Figure 1A). Once these cardiac pools (first heart field, FHF) migrate towards the embryonic anterior ventral midline, they give rise to the cardiac crescent and then to a linear heart tube, constituted of endocardium (inner lining) and myocardium (outer lining) (Staudt and Stainier, 2012; Schleich et al., 2013; Kelly et al., 2014; D. R. Brown et al., 2016) (Figure 1B-D, Figure 2). Subsequently, late differentiating progenitor cells (second heart field, SHF) derived from the pharyngeal mesoderm migrate toward the poles of the heart tube. The progressive addition of these cells drives the continuous extension of the heart, contributing to the 
expansion of the heart tube and the formation of the outflow tract (OFT) and sinus pole (Mjaatvedt et al., 2001; Zhou et al., 2011; Kelly et al., 2014; Knight and Yelon, 2016; Felker et al., 2018) (Figure 2).

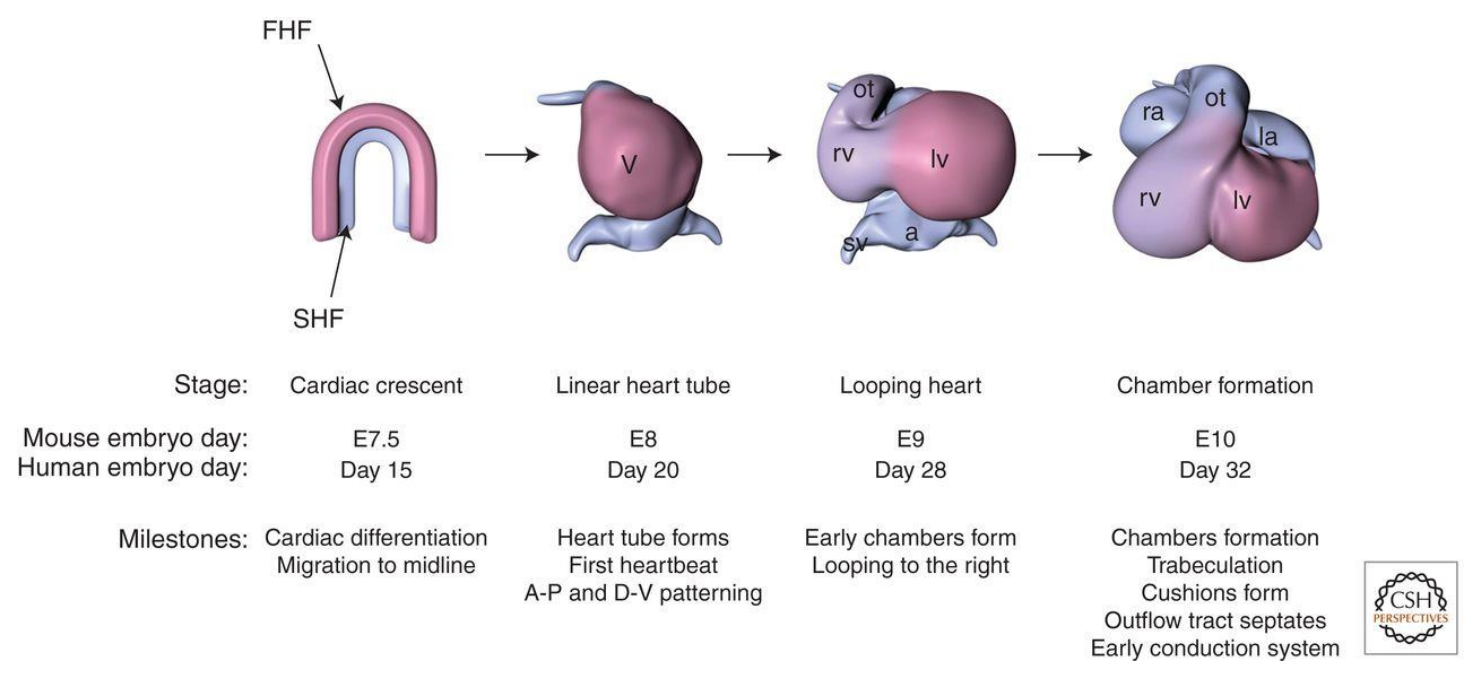

Figure 2: Schematic representation of mammal heart development.

Heart development starts with the formation of the cardiac crescent and the migration of the first heart field (FHF) and second heart field (SHF) precursors to the midline. The linear tube forms around E8/Day20 and starts to beat, then it loops (E9/Day28) and forms the chambers. At late stages, several events take place such as trabeculation, cushions, and conduction system formation. (A-P, anteroposterior; D-V, dorsoventral; rv/lv and ra/la, right and left ventricle or atria; sv, sinus venosus; ot, outflow tract). Image reprinted from Cold Spring Harb Perspec Biol (Bruneau, 2013) Figure 1, with permission from Cold Spring Harbor Laboratory Press.

Subsequently, as the linear heart tube elongates, it undergoes morphogenetic movements "jogging" and "looping" - that contribute to the characteristic left-right asymmetry of the heart (Srivastava, 2006; Bruneau, 2013; Desgrange et al., 2018) (Figure 2). At the onset of looping, the two chambers and the OFT start to grow and proliferate, increasing their volume and bulging out of the heart tube in a process termed "ballooning" (de Boer et al., 2012; Kelly et al., 2014) (Figure 3). After these processes correctly position the atrium and the ventricle, several additional morphogenetic events further contribute to the maturation and size increase of the heart (Bruneau, 2013; Schleich et al., 2013) (Figure 3).

These processes include: the formation of the trabeculae, finger-like projections that protrude inside the chambers and increase cardiac pumping; the formation of the cardiac valves, which ensure unidirectional blood flow; and the maturation of the conduction system. At the same time, in mammals, the septation of the chambers takes place, dividing atria and ventricles with appositely forming septa, ensuring the separation of the oxygenated and non-oxygenated blood. The OFT also undergoes septation, cushion formation to form the valves, and rotation, giving rise to the roots of the great arteries and the aortic arches (Rothenberg et al., 2003; Bajolle et al., 2006). 


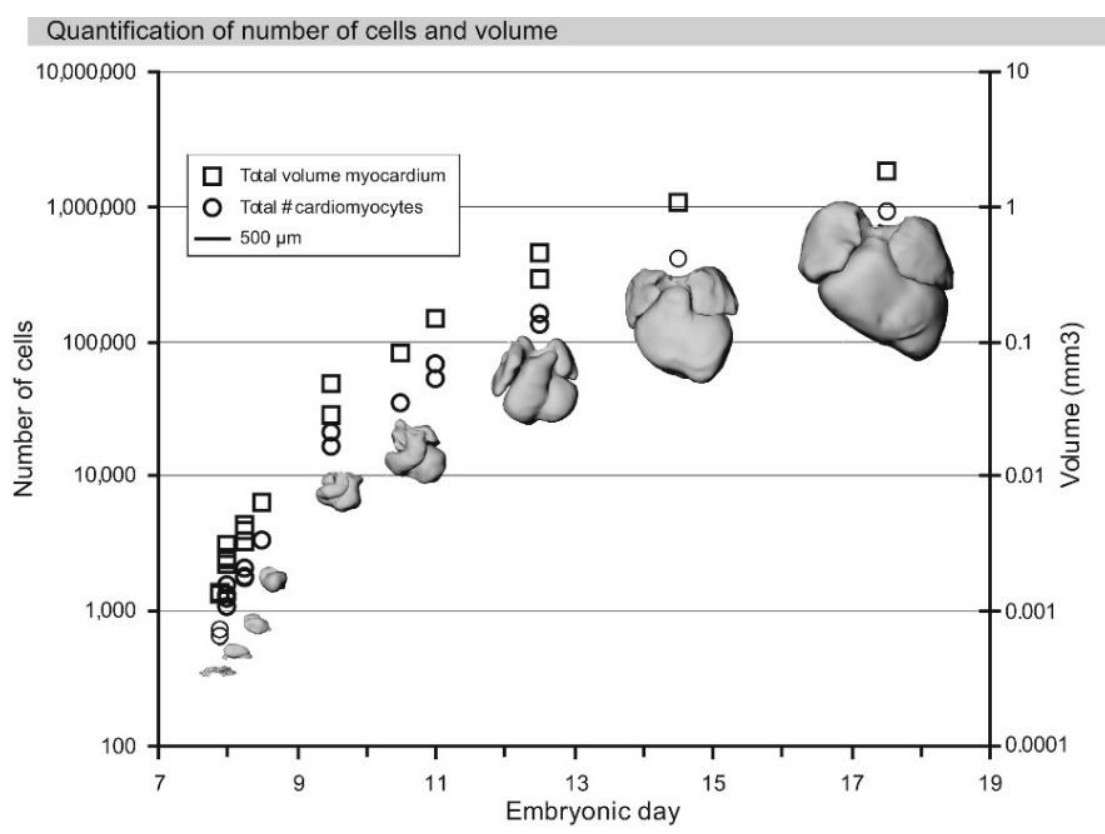

Figure 3: Graph depicting the increase in volume and cell number during mammalian heart development.

Heart development is accompanied by an increase in volume and cardiomyocyte proliferation. The steepest growth happens between E7.5 and E10, and then it slows down in the late stages of heart development. Image reprinted from Developmental Biology (de Boer et al., 2012) Figure 2, with permission from Elsevier.

Once developed, the OFT gives rise to the bases of the aorta and the pulmonary artery in adults, with the respective valves. In vertebrate hearts with only one ventricle, where the chambers do not undergo septation, the OFT does not divide but instead forms a subsidiary "third chamber", the BA (Grimes and Kirby, 2009; Zhou et al., 2011; Guner-Ataman et al., 2013; Knight and Yelon, 2016; Paffett-Lugassy et al., 2017). The BA persists until adulthood, sitting between the heart and the vast vascular network, buffering the blood pressure deriving from the ventricles. The main function of both muscular arteries in mammals and BA in fish is their cushioning role, which transforms pulsatile flow deriving from the heart into a steady flow in the vascular circulation.

\subsection{Cell-types forming the heart and their origin}

Several pools of cardiovascular cell types are needed to build and maintain such a complex organ, and cardiac muscle cells only account for less than $30 \%$ of the total number of cells (Meilhac et al., 2014; Bergmann et al., 2015; Perbellini et al., 2018) (Figure 4).

As mentioned above, at the linear heart tube stage, the heart is composed of mainly two layers: an interior layer of endocardial cells (ECs), and an exterior layer of cardiomyocytes (CMs), separated by extracellular matrix (ECM) (Schleich et al., 2013; Kelly et al., 2014). These cell types are derived mainly from the FHF and SHF and serve different roles. The endocardium is a regionally specialized endothelium that lines the lumen of the heart, deriving from a distinct 
population of mesodermal cardiac precursors in the cardiac crescent (Misfeldt et al., 2009; Harris and Black, 2010; Milgrom-Hoffman et al., 2011). It integrates the mechanical signals induced by fluid flow, is exposed to nutrients and signaling cues in the blood, regulates cardiomyocyte function, embeds the cardiac conduction system, and is responsible to form the valve endocardial cushions (Beis et al., 2005; Bressan et al., 2014; Milgrom-Hoffman et al., 2014; Haack and Abdelilah-Seyfried, 2016; Rasouli and Stainier, 2017; Gunawan et al., 2019). The myocardium is an involuntary, striated muscle that constitutes most of the cardiac volume. It is composed of CMs, contracting cells that allow the heart to beat. Their function relies on sarcomeres, the functional unit of these cells, formed by actomyosin myofibrils. Other than ensuring correct pumping by contracting, cardiomyocytes contribute to the proper morphogenesis of the embryonic heart, by changing their shape, proliferating, and delaminating (Buckingham et al., 2005; Auman et al., 2007; Uribe et al., 2018). Their behavior is dictated mainly by biomechanical cues (Y. F. Lin et al., 2012; Priya et al., 2020), but signaling interactions with the neighboring tissues also play a fundamental role in their development and function (Grego-Bessa et al., 2007; J. Liu et al., 2010; Han et al., 2016; Dogra et al., 2017; Colliva et al., 2020).

Subsequently, starting at E9-E9.5 in mouse and $52 \mathrm{hpf}$ in zebrafish, another cell type starts covering the chambers, the epicardium (Manasek, 1969). It initially forms as an extra cardiac proepicardial organ (PEO), an aggregate of cells situated dorsal to the developing heart (Manner et al., 2001; Simoes and Riley, 2018; Quijada et al., 2020). The PEO is thought to derive from pronephric external glomeruli, present throughout evolution since the lampreys (Pombal et al., 2008). Eventually, the epicardium delaminates from the PEO and the single cells contact the myocardial surface, flatten, and start proliferating. Eventually, they will cover all the cardiac chambers and contribute to the formation of coronary vessels and fibroblasts in the adult hearts (Simoes and Riley, 2018; Quijada et al., 2020) (Figure 4).

The arterial pole of the heart, which is mainly SHF-derived (Mjaatvedt et al., 2001; Zhou et al., 2011; Knight and Yelon, 2016) and gives rise to the OFT, is also colonized by smooth muscle cells of SHF origin, and then covered by epicardial cells. Vascular SMCs (vSMCs), a major structural component of the vessel wall, are involuntary, smooth muscles that line the ECs of the bigger vessels (mainly arteries). Their major structural role is to regulate vascular tone and maintain intravascular pressure throughout the organism, distending during systole and recoiling during diastole (Neeb et al., 2013; G. Wang et al., 2015; Lacolley et al., 2017).

During development, vSMCs are recruited via several chemoattractants (e.g., platelet-derived growth factor (PDGF)-BB or transforming growth factor- $\beta$ (TGF- $\beta$ ) after the formation of the vessels and are crucial for the final maturation of the vascular system (De Val, 2011; Salabei et al., 2013; Stratman et al., 2017) (Figure 4). During the embryonic state, vSMCs are actively proliferating and migrating, but also secrete a large amount of the extracellular matrix 
composing the vessel wall. Once mature, they start expressing more specific markers (e.g., $\alpha-$ smooth muscle actin, Acta2/ $\alpha$-SMA) and switch their phenotype from a proliferating to a contractile one (Owens, 1995; Owens et al., 2004; Salmon et al., 2012). This phenotypic switch is essential to form a mature vessel wall, but it needs to be temporally fine-tuned to ensure the initial complete coverage of the vessel.

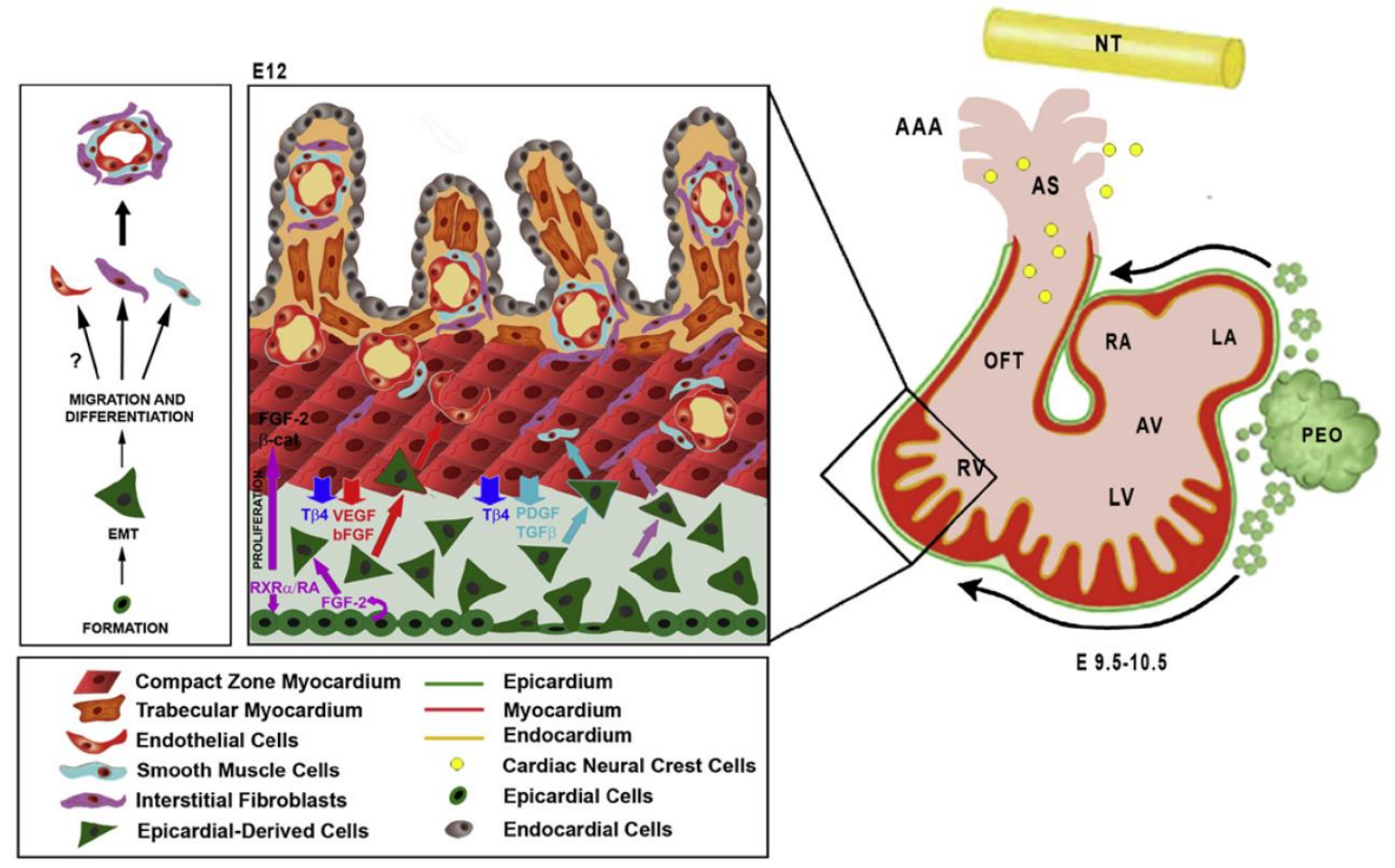

Figure 4: Schematic representation of cellular composition of the developing heart.

Around E9.5 the epicardial cells start delaminating from the proepicardium and cover the cardiac chambers; at the same time neural crest cells migrate from the neural tube and colonize the OFT. At E12, the heart is composed mainly by compact and trabecular myocardium, endocardial/endothelial cells lining the lumen and forming the coronaries, smooth muscle cells covering the coronaries and the OFT, epicardial and epicardial derived cells, and cardiac neural crest. (NT, neural tube; RV/LV and RA/LA, left/right ventricles and atria; AV, atrioventricular canal; OFT, outflow tract; PEO, proepicardium; AA, aortic arches). Image reprinted from Cell Stem Cells (Martin-Puig et al., 2008) Figure 6, with permission from Elsevier.

All the cells described above are of mesodermal origin. Another type of cells that contribute to the morphogenesis of the heart are the ectoderm-derived neural crest cells (NCCs) (Kirby, 2001) (Figure 4). These cells colonize the heart when the chamber formation is complete (zebrafish: $4 \mathrm{dpf}$; mouse: E10.5) and play an important role in the maturation of the arterial pole of the heart and the great vessels, contributing to the septation of the OFT and providing additional SMCs (Hutson and Kirby, 2003; Stoller and Epstein, 2005; Srivastava, 2006; Cavanaugh et al., 2015; Plein et al., 2015). 


\subsection{Communication between different cell types}

The distinct cell pools described in the previous section are not isolated from one another; they sense different stimuli from the environment and integrate their biological responses. To do so, cells and tissues interact physically and via a variety of soluble factors, to ensure proper development and functional homeostasis of the heart (Tirziu et al., 2010; Fountoulaki et al., 2015). Different paths are used by the cells to ensure efficient communication with each other (Figure 5).

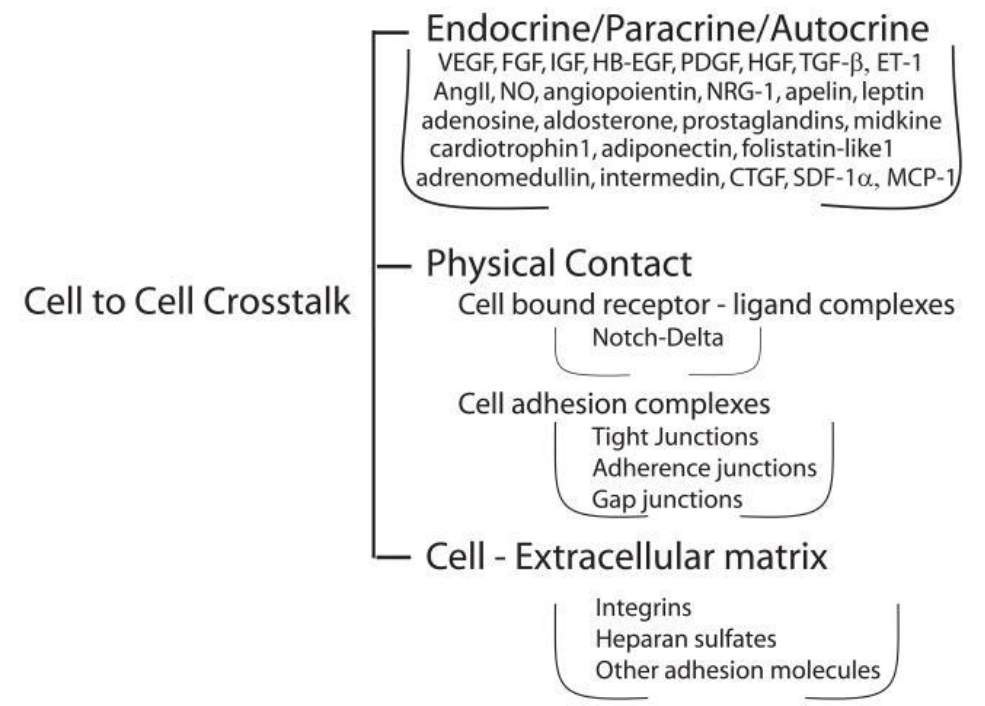

Figure 5: Schematic representation of cellular crosstalk in the heart.

Cell-cell communication takes place in different ways: secretion of soluble factors, which act in endocrine/paracrine/autocrine manners; physical contact, via membrane-bound ligandreceptors or cellular junctions; mediation of the ECM. Image reprinted from Circulation (Tirziu et al., 2010) Figure 1, with permission from Wolters Kluwer Health, Inc.

One way in which two neighboring cells can transfer information relies on their physical interactions (Fountoulaki et al., 2015). Cellular crosstalk can happen through cell-cell contact, mediated by gap, adherent, or tight junctions. Alternatively, membrane-bound ligand-receptor couples (e.g., Notch or Ephrins) can activate downstream signaling pathways in the contacting cell (Lai, 2004; MacGrogan et al., 2018). Conversely, when cells are not adjacent to each other, they rely on the secretion of factors into the bloodstream or their deposition in the ECM. Cells secrete ligands belonging to several families such as vascular endothelial growth factor (VEGF), fibroblast growth factor (FGF), bone morphogenetic protein (BMP)/ TGF- $\beta$, neuregulins (NRG), nitric oxide (NO), Apelin, retinoic acid (RA), PDGF, angiopoietins, and several others (Tirziu et al., 2010; Fountoulaki et al., 2015). These ligands subsequently bind the respective receptor on the receiver cell and activate downstream intracellular signaling pathways.

Another way for cells to be in contact with each other is through the ECM, a complex meshwork of fibers, composed of secreted factors such as glycoproteins, collagens, fibronectins, laminins, glycosaminoglycans and proteoglycans (Kim et al., 2011). It constitutes the scaffold that supports the physical structure of many organs, providing tissue integrity and positioning, but its view as an inert structural framework has been increasingly challenged (Kim et al., 2011; Valiente-Alandi et al., 2016; Silva et al., 2020; Derrick and Noel, 2021). It is now 
thoroughly accepted that the ECM plays a crucial role in establishing the correct extracellular microenvironment needed by cells to proliferate, migrate and differentiate, and by organs to grow and maintain their homeostasis (Valiente-Alandi et al., 2016; Derrick and Noel, 2021). Its ways of facilitating these processes can be purely mechanical or signaling-mediated (Kim et al., 2011). For example, cells can sense variations in ECM stiffness and elasticity due to the different proportion of several components (Sapir and Tzlil, 2017). These differences can either mechanically affect cellular movements, altering the attachment of the cells to the matrix, or even influence gene expression and signaling. Cells largely respond to these changes via the crosstalk between integrins (bound to several ECM components) and the cellular actin cytoskeleton, mediated by focal adhesion factors or by hemidesmosomes (Hynes, 2007; Kim et al., 2011). In addition, the ECM also exerts its effects on organ growth and homeostasis by modulating signaling pathways activity, serving as a reservoir of growth factors. Secreted factors such as TGF- $\beta$, BMP, and FGFs, are sequestered in the ECM, which acts as a local reservoir, and released by proteases when needed, to provide a temporally fine-tuned concentration (Kim et al., 2011; Valiente-Alandi et al., 2016).

\subsection{The OFT as a model to study cell interactions}

As mentioned above, the heart is composed of an incredibly high amount of cell types of different origins that communicate and contribute to the correct formation of this complex organ. Among all the regions of the heart, the arterial pole, which sits at the interface between the cardiac compartment and the vascular network (Kelly and Buckingham, 2002; Sugishita et al., 2004), condenses several cell type progenitors with different origins in a confined space. The development of the OFT starts with the formation of a ventricular constriction lined by ECs and enclosed by CMs, both originated from SHF progenitors (Felker, et al., 2018; PaffettLugassy, et al., 2017; Kelly and Buckingham, 2002). Later, this tube becomes covered by SMCs of SHF and neural crest origin. This intricate cellular coordination is essential for the dynamic development of the OFT and makes the OFT susceptible to several developmental defects, which account for one-third of all congenital heart defects (CHD) in human patients (Neeb, et al., 2013). Some of these malformations are the persistent truncus arteriosus (PTA), overriding aorta (OA), transposition of the great arteries (TGA), as well as dilation or coarctation of the great arteries (Anderson, et al., 2016; Neeb, et al., 2013). To explain these pathologies, much attention has been given to the prevalent cell types forming the OFT, SMCs and CMs (Kelly and Buckingham, 2002; Waldo, Hutson, Ward, et al., 2005; Hami et al., 2011). In addition, in mammalian and avian models, CNCs have been proven to be essential for OFT septation, and their misalignment is responsible for several of the congenital defects (Kirby, 2001; Waldo, Hutson, Stadt, et al., 2005; Darrigrand et al., 2020). Nonetheless, a prominent cell type in the OFT, which also contributes to formation of the valves, is the endocardium/endothelium. The 
close contact of ECs with SMCs makes their crosstalk key for development and homeostasis (Lilly, 2014; Stratman et al., 2017; Segers et al., 2018). The interplay of these two cell types is also confirmed by the presence of several complementary ligand-receptor couples on the two sides (Gaengel et al., 2009; Sweeney and Foldes, 2018).

While SMCs are needed to stabilize the vascular network, endothelial cells are initially required to recruit SMCs and later to influence their proliferation and differentiation state. Moreover, these two cell types both contribute to the extracellular environment in which SMCs are embedded. While being thoroughly studied in other contexts including the formation of the vessels or the main chambers of the heart, the role of ECs in the development of the OFT has so far largely been neglected. Only a few recent studies proposed a potential role for the endothelium in several pathologies affecting the great arteries (L. Sun et al., 2008; van de Pol et al., 2017).

\subsubsection{TGF $\beta$ signaling and its various roles during embryonic development}

Another level of complexity in understanding OFT development and disease comes from the intricate crosstalk of several pathways. Multiple signaling cues have been implicated in its correct morphogenesis, such as BMP, Notch, FGF, Wnt, RA, and TGF $\beta$ (Neeb, et al., 2013). TGF $\beta$ signaling pathway is one of the most important pathways during embryonic development. During evolution, the pathway emerges with the first multicellular organisms, and its ligands and downstream components are highly conserved across metazoans. Its roles during development include, but are not limited to, regulation of gastrulation and specification of the dorso-ventral axis, control of cell migration, differentiation, proliferation, cell death and survival, and ECM production (Wharton and Derynck, 2009; M. Y. Wu and Hill, 2009; Massague, 2012) (Figure 6). Moreover, the TGF $\beta$ pathway exerts prominent roles in maintaining organ homeostasis, and mutations in its components are often implicated in diseases.

The TGF $\beta$ pathway is often associated with the BMP pathway because their ligands belong to the common TGF $\beta$ superfamily, comprising 30 different growth factors involved in numerous processes. For the scope of this work, in the next section I will focus on the mechanisms specific to the canonical TGF $\beta$ pathway (i.e., mediated by Smads), but the main steps of the signaling are also conserved for the canonical BMP. 


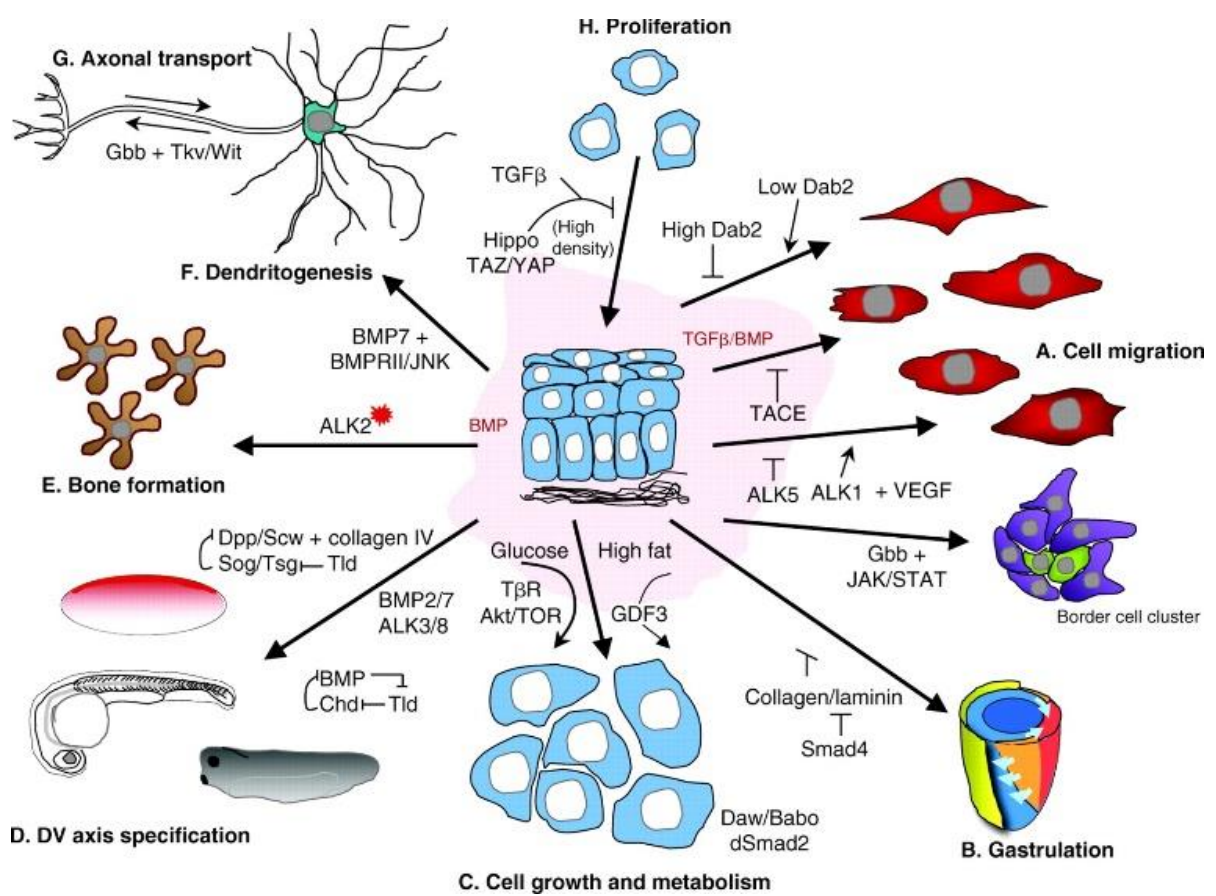

Figure 6: Schematic representation of biological responses to TGF- $\beta$ family members during embryonic development.

TGF $\beta$ has proved to be a crucial signaling pathway involved in multiple contexts during embryonic development, including individual and cluster cell migration (A), control of extracellular environment during gastrulation $(\mathbf{B})$, cell size increase regulated by metabolic processes $(\mathbf{C})$, specification of the dorso-ventral axis (D), bone deposition $(\mathbf{E})$, dendritogenesis and axonal transport $(\mathbf{F}, \mathbf{G})$, and cell proliferation $(\mathbf{H})$. Image reprinted from Development (Wharton and Derynck, 2009), Figure 2, with permission from The Company of Biologists Ltd.

\subsubsection{Mechanistic insights into the TGF $\beta$ pathway}

The ligands of the TGF $\beta$ superfamily are synthesized as precursors, comprising a long prodomain (LAP, latency-associated polypeptide) and the mature polypeptide. Soon after synthesis, the LAP gets cleaved but remains covalently associated with the mature ligand (Figure 6). Two ligand proteins dimerize through the creation of disulfide bonds and are secreted in the ECM as dimeric precursors. These dimeric complexes can associate with latent TGF- $\beta$ binding proteins (LTBP) and in turn bind to ECM components (i.e., collagen, fibulins, fibronectin, and fibrillins). The ECM serves as a reservoir and prevents premature TGF- $\beta$ activation and binding to receptors. In response to stimuli, the ligands get activated by cleavage and released from the ECM, processes that happen in a cell- and context-specific manner (Derynck and Budi, 2019).

Once activated, ligand dimers can bind to tetrameric receptor complexes located on the plasma membrane.

The TGF- $\beta$ pathway comprises different receptors, mainly divided into two groups, type I and type II, sharing sequence and structural similarities. Both receptors are serine-threonine kinases, with an ectodomain, a transmembrane domain, and a cytoplasmic kinase domain. Specifically, 
in type I receptors, a juxtamembrane glycine-serine-rich sequence (GS domain) is located between the transmembrane domain and the kinase domain. The combination of different ectodomains of different receptors ensures ligand binding and signaling specificity (Feng and Derynck, 2005; Derynck and Budi, 2019). Receptors type II (mainly Tgfbr2) are constitutively phosphorylated dimers and are the first ones bound by the ligands, because of their higher affinity. Ligand binding triggers the heterotetramerization of Tgfbr2 dimers with two TGF- $\beta$ receptors type I (i.e., Tgfbr1/Alk5, Alk1, Alk7). Upon tetramerization, Tgfbr2 receptors phosphorylate the GS domain of type I receptors.

Several mechanisms are in place to prevent premature activation of the signaling in the absence of a ligand, such as the binding of the immunophilin FKBP12 to the type I receptors, or the inhibitory intracellular Smads (I-Smads; Smad7 for TGF- $\beta$ ) (Feng and Derynck, 2005; Derynck and Budi, 2019). Upon receptor activation, receptors type I phosphorylate the intracellular main TGF- $\beta$ effectors, named Smads (Figure 6). Smad 2 and Smad 3 are the TGF- $\beta$ receptoractivated Smads (R-Smads). Once phosphorylated, they detach from the receptor complex and associate to Smad4, forming trimers (2 R-Smads and 1 Smad4). As a complex, they translocate to the nucleus where they bind DNA and activate or repress gene expression (Figure 6).

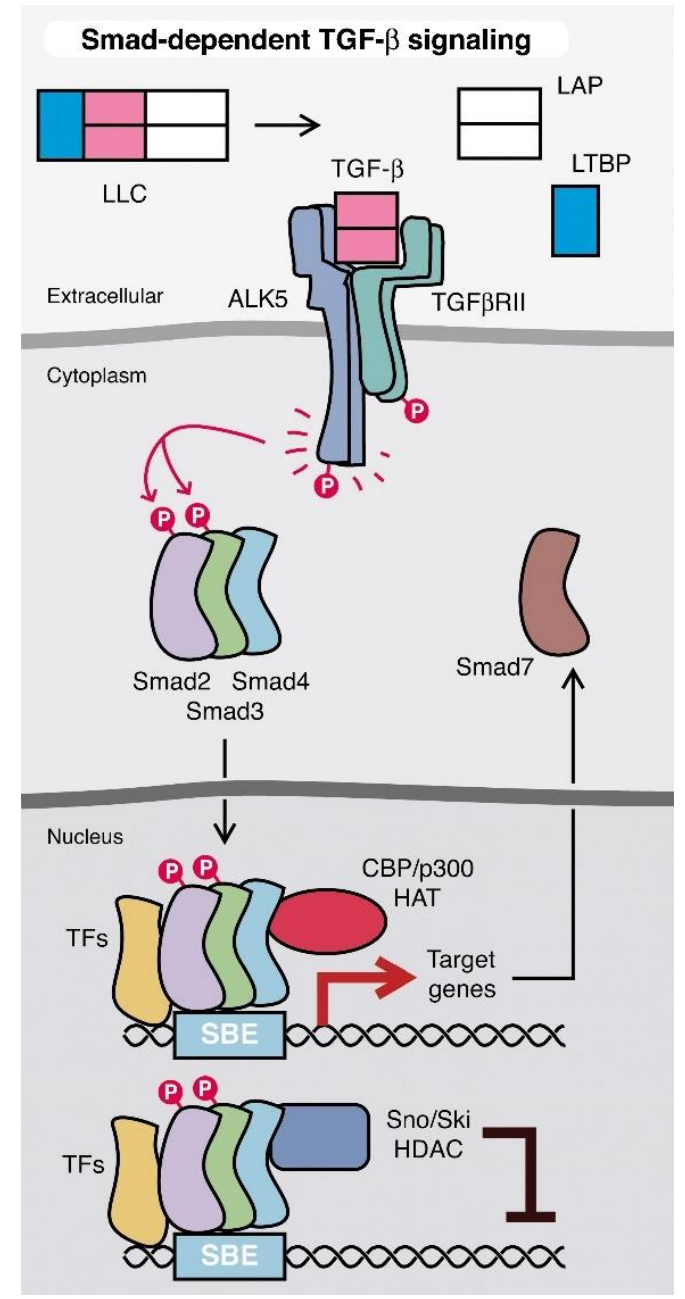

Figure 6: Schematic representation of the TGF- $\beta$ pathway.

The TGF $\beta$ signaling activates with the cleavage and activation of the ligands and their binding to the membrane-bound receptors. The receptors have the ability to phosphorylate the intracellular Smads $(\operatorname{Smad} 2 / 3$, which become P-Smad2/3), which bind Smad4 and translocate to the nucleus. There, together with multiple cofactors, the Smads bind to Smad-binding elements (SBE) on the promoter of target genes (among which we find the inhibitor of the pathway, Smad7). When the pathway needs to stay in an inactive state, PSmads bind the inhibitors Ski/Sno that prevent the activation of target genes. (LAP, Latency Associated Peptide; LLC, large latent complex; LTBP, latent transforming growth factor $\beta$ binding proteins; TFs, transcription factors; HDAC, histone deacetylase; HAT, histone acetyltransferase). Image adapted from Annu. Rev. Immunol. (M. O. Li et al., 2006), Figure 2, with permission from Annual Reviews. 
Together with their transcription factor activity, Smads are involved in the regulation of mRNA splicing, miRNA processing, and epigenetic modifications (Derynck and Budi, 2019). Most of the time, Smads do not act alone but need the cooperation of several cofactors, due to their low-affinity binding to DNA. This association is especially crucial for Smad2, which lacks a proper DNA-binding domain (Hill, 2016; Aragon et al., 2019). The selectivity of the Smad activation relies on the type I receptors to which the R-Smads are recruited. Each Smad can bind its own cofactors and regulate different gene expression, exerting a distinct cellular function, often antagonizing each other (Phanish et al., 2006; K. A. Brown et al., 2007; Hill, 2016; Dogra et al., 2017; Derynck and Budi, 2019).

Adding another layer of complexity, several more activatory and inhibitory loops characterize the TGF- $\beta$ signaling pathway to ensure its spatiotemporal activation, signaling robustness, duration, and plasticity (Yan et al., 2018; Derynck and Budi, 2019). Among these, the activity of Ski/Sno proteins, which antagonize Smad activation by regulating their binding to DNA, is crucial (Stroschein et al., 1999; Tecalco-Cruz et al., 2018). Moreover, the regulation of the expression and function of several pathway component genes is regulated by feed-forward and feedback loops dictated by the activation of the pathway. For instance, the pathway upregulates the expression of ECM proteins participating in the ligand activation, or TGF- $\beta$ receptor inhibitors such as Smad7 are positively regulated by the TGF- $\beta$ pathway itself (Yan et al., 2018)

\subsubsection{The TGF $\beta$ pathway in cardiovascular development}

The TGF- $\beta$ signaling pathway exerts several tissue-dependent biological functions, and its regulation of cardiovascular development and homeostasis is one of the most prominent, as evidenced by genetic studies in mice and humans.

Indeed, mutations of most of the signaling component genes results in embryonic lethality due to the alteration of the cardiovascular system. Even heterozygous mutations are often responsible for defects in adult patients, such as arteriovenous malformations (AVMs), atherosclerosis, aortic aneurysms, cardiac fibrosis, and valvular diseases (Figure 7).

Unfortunately, the multifunctional, cell- and context-dependent activities of TGF- $\beta$ have complicated the understanding of its in vivo roles in cardiovascular biology. Relying on in vitro models also failed to explain its cell-specific functions, often resulting in contradictory results depending on the cell line, environmental variables, and confluence of the cells.

One of the best known biphasic roles of TGF- $\beta$ takes place in endothelial cells. In particular, TGF- $\beta$ was shown to both promote and inhibit angiogenesis in different contexts, depending on the specific receptors (Goumans et al., 2002; Goumans, Lebrin, et al., 2003; Seki et al., 2006; Aspalter et al., 2015; Maring et al., 2016; Rochon et al., 2016). In particular, the signaling through Alk1 seems to promote the proliferation and migration of ECs, while Alk5 activation 
is thought to antagonize those processes (Larsson et al., 2001; Goumans et al., 2002; Lamouille et al., 2002; Goumans, Lebrin, et al., 2003; Carvalho et al., 2007; Cunha et al., 2010; Aspalter et al., 2015). Notably, Alk5 is also required for Alk1-mediated signaling, complicating the interpretation of the results (Goumans, Valdimarsdottir, et al., 2003).

\begin{tabular}{|c|c|c|c|}
\hline Gene & Human disease & Animal model & Reference \\
\hline \multicolumn{4}{|l|}{ Ligands } \\
\hline TGF $\beta 1$ & $\begin{array}{l}\text { Camurati-Engelmann } \\
\text { disease }\end{array}$ & $\begin{array}{l}\text { KO: embryonic lethal with vascular defects or postnatal lethality } \\
\text { from autoimmune disease. }\end{array}$ & [13-16] \\
\hline TGF $\beta 2$ & Kawasaki disease & KO: aortic arch defects, cardiac septal defects, perinatal lethality. & {$[17,18]$} \\
\hline TGF $\beta 3$ & unknown & KO: cleft palate, delayed lung maturation, die shortly after birth. & {$[19,20]$} \\
\hline \multicolumn{4}{|l|}{ Receptors } \\
\hline $\mathrm{T} \beta \mathrm{RII}$ & MFS2, LDS & KO: embryonic lethal, vascular defects & {$[21,136]$} \\
\hline T $\beta R I / A L K 5$ & LDS & KO: embryonic lethal, angiogenesis defects & {$[22,145,146]$} \\
\hline ALK1 & HHT & $\begin{array}{l}\text { KO: embryonic lethal, reduced VSMC differentiation, dilated ves- } \\
\text { sels, AVMs }\end{array}$ & {$[23,24]$} \\
\hline BMPRII & $\mathrm{PAH}$ & $\begin{array}{l}\text { KO: pre-angiogenesis lethality } \\
\text { Transgenic BMPR2-mutant allele: pulmonary hypertension }\end{array}$ & [25-27] \\
\hline \multicolumn{4}{|l|}{ Accessory receptors } \\
\hline ENG (endoglin) & HHT & $\begin{array}{l}\text { KO: embryonic lethal due to vascular defects, reduced vSMC dif- } \\
\text { ferentiation, heart defects. } \\
\text { Het: vascular lesions similar to HHT. }\end{array}$ & [28-30] \\
\hline Soluble Endoglin & Pre-eclampsia & & {$[51]$} \\
\hline TGFBR3 (betaglycan) & Familial intracranial aneurysm & $\begin{array}{l}\text { KO: poorly formed cardiac septa, incomplete compaction of ven- } \\
\text { tricular walls }\end{array}$ & {$[31]$} \\
\hline \multicolumn{4}{|l|}{ Smads } \\
\hline Smad1 & Unknown & KO: embryonic lethal due to defects in chorion-allantoic circulation & {$[32]$} \\
\hline Smad3 & Aortic aneurysm-osteoarthritis & & [33-37] \\
\hline Smad4 & JP and HHT & KO: embryonic lethal & {$[38-40]$} \\
\hline Smad5 & Unknown & KO: embryonic lethal due to angiogenesis defects & [41-43] \\
\hline Smad6 & Unknown & $\begin{array}{l}\text { KO: heart abnormalities, aortic ossification and elevated blood } \\
\text { pressure }\end{array}$ & {$[44]$} \\
\hline Smad7 & Unknown & KO: embryonic lethal due to cardiovascular defects & {$[45]$} \\
\hline Smad8 & $\mathrm{PAH}$ & Defective pulmonary vascular remodeling & {$[46]$} \\
\hline
\end{tabular}

Abbreviations: KO, knockout; Het, heterozygous; AVMs, arteriovenous malformations; LDS, Loeys-Dietz syndrome; MFS2, Marfan syndrome type 2; HHT, hereditary haemorrhagic telangiectasia; PAH, pulmonary arterial hypertension; JP, Juvenile polyposis; VSMC, vascular smooth muscle cell.

Figure 7: Mutations in genes encoding components of the TGF- $\beta$ pathway lead to cardiovascular alterations in human and mouse.

List of human diseases and cardiovascular defects in the animal models, caused by mutation in several components of the TGF- $\beta$ pathway. Image reprinted from Trends in Cell Biology (Pardali and Ten Dijke, 2012), Table 1, with permission from Elsevier.

In addition to its role in angiogenesis, TGF- $\beta$ is also one of the major players in endocardial cushion and valve formation, homeostasis, and regeneration (Goumans and Ten Dijke, 2018; Bensimon-Brito et al., 2020) by regulating endothelial-to-mesenchymal (EndoMT) (Azhar et al., 2009; Azhar et al., 2011; Goumans and Ten Dijke, 2018). Cells undergoing EndoMT lose EC markers while acquiring expression of mesenchymal cell markers, including $\alpha$-SMA, transgelin, vimentin, and collagen. Excessive EndoMT is also responsible for a myriad of pathologies, such as cerebral cavernous malformations or cardiac fibrosis, in which dysregulation of TGF- $\beta$ is often involved (Goumans et al., 2008; Azhar et al., 2009; Maddaluno et al., 2013; Cuttano et al., 2016; Goumans and Ten Dijke, 2018). 
In addition to its function in ECs, one of the best-studied functions of TGF- $\beta$ is regulating SMCs (Pardali et al., 2010; Pardali and Ten Dijke, 2012; Goumans and Ten Dijke, 2018). While its role in proliferation and apoptosis is debated due to conflicting results (Hishikawa et al., 1999; Seay et al., 2005; Suwanabol et al., 2012; Shi et al., 2014), TGF- $\beta$ function in differentiation is well recognized and depends on the activation of SMC marker genes, such as a-SMA, Lysiloxidase, Elastin and Transgelin (S. Chen et al., 2003; Hu et al., 2003; Choudhary et al., 2009; Fadini and Tjwa, 2010; Guo and Chen, 2012; Jaffe et al., 2012).

In particular, it has been observed that mutations in TGF- $\beta$ pathway components often lead to the development of pathologies of the great arteries, such as aortic aneurysms and dissection (TAAD). Moreover, Loeys-Dietz (LDS) and Marfan syndromes (both exhibiting dilation and weakness of the aorta and the OFT) are mainly caused by mutations in several TGF- $\beta$ genes (e.g., Alk5, Smad2/3, and Tgfbr2) (Loeys et al., 2005; Loeys et al., 2006; Matyas et al., 2006; F. Lin and Yang, 2010; Brownstein et al., 2017; Camerota et al., 2019).

Aortic aneurysms are associated with the loss of the aortic wall structure, mainly caused by SMC apoptosis, reduced migration, impaired ECM deposition, resulting in the dissection of the vessel (Perrucci et al., 2017; Michel et al., 2018; Takeda et al., 2018; Jana et al., 2019). Recently, two studies started evaluating a therapeutic role for the endothelium in these diseases (van de Pol et al., 2017; J. Sun et al., 2018). Therefore, until now the correlation between TGF- $\beta$ and aneurysms has revolved around its role in SMC behavior (F. Lin and Yang, 2010; Gillis et al., 2013; Renard et al., 2013; W. Li et al., 2014; P. Yang et al., 2016; Perrucci et al., 2017; Clift and Cervi, 2020).

\subsubsection{The role Alk5/Tgfbr1 in heart development and aortic aneurysm formation}

As mentioned above, Tgfbr1/Alk5 is the main type I receptor of the TGF- $\beta$ signaling pathway, and its mutations are among the main causes of LDS and Marfan syndromes, as well as TAADs. However, its role during cardiac development is still not completely understood. During embryonic murine development, Alk.5 is expressed preferentially in the aorta and the heart, particularly in the SMC layer (Seki et al., 2006). A global mutation of Alk.5 leads to embryonic death at E11-12 due to cerebral hemorrhage and supposed cardiac failure (Carvalho et al., 2007)

(Figure 8a). Notably, only endothelial-specific Alk.5 mutants exhibit a similar phenotype (Sridurongrit et al., 2008) (Figure 8b). Conversely, mutation of Alk.5 specifically in pericytes or SMCs does not lead to any observed defect in the developing heart or great vessels (Sridurongrit et al., 2008; Dave et al., 2018). 
a
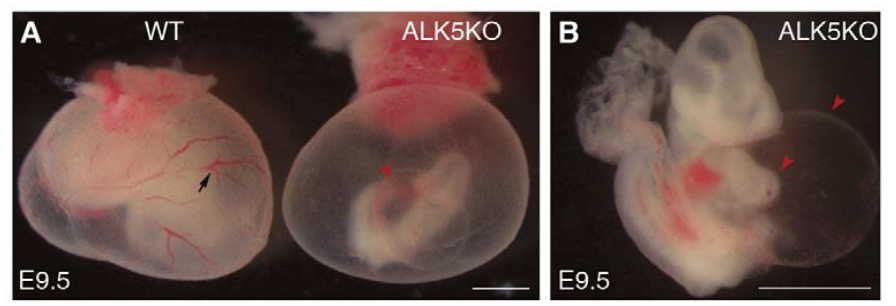

b
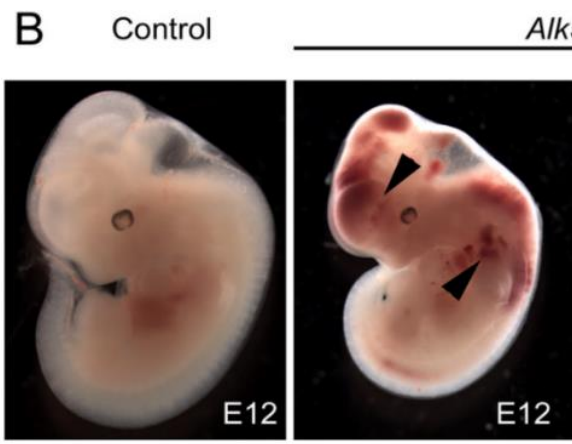

Alk5/Tie2-Cre

Figure 8: Mutations in Alk5 lead to cardiovascular abnormalities and embryonic lethality.

Both global (a) and endothelial-specific Tie2-Cre driven (b) Alk.5 knock-out mice die within E12 of embryonic development. In both cases, the embryos display a lack of blood vessels covering the yolk sack, accompanied by pericardial edema, hemorrhage in the brain, and presumed cardiac insufficiency. Image reprinted from a) (Carvalho et al., 2007) Figure 1, and b) (Sridurongrit et al., 2008) Figure 1, with permissions from The Company of Biologists Ltd. and Elsevier, respectively.

In spite of the evidence for a conceivable role for Alk5 in ECs during development, a thorough investigation of later phenotypes has been hindered by the early lethality of Alk.5 homozygous mutants. Notably, while heterozygous animals do not exhibit any developmental defect, they are prone to aneurysm development in adult stages, suggesting Alk.5 haplosufficiency only during development (Loeys et al., 2005; Loeys et al., 2006; P. Yang et al., 2016). Thus, to date, the attention has been focused on the diseases appearing in adult stages and on Alk5 role in SMCs (Choudhary et al., 2009; Guo and Chen, 2012; Gillis et al., 2013; P. Yang et al., 2016; Takeda et al., 2018).

\subsection{Another hub of interactions: the epicardium-myocardium surface}

\subsubsection{The role of the epicardium during development and regeneration}

The epicardium is the mesothelial layer that envelops the heart. It is the last layer to be added to the heart during development, and it originates from the PEO, a transitory extracardiac embryonic structure. As previously described, epicardial cells delaminate from the PEO and, in both mouse and zebrafish, attach to the naked myocardium as free-floating cells due to physical constraints. The site of adhesion on the myocardium seems to be influenced by the 
flow of pericardial fluids (Peralta et al., 2013). After the attachment to the myocardium and the formation of a complete mesothelial layer enveloping the heart, the epicardium starts undergoing epithelial-to-mesenchymal transition (EMT; E12.5 in mouse) (Smith et al., 2011; Acharya et al., 2012). These cells lose their apicobasal polarity and cell junctions, and they acquire a migratory behavior, giving rise to several cells embedded in the myocardium, known as epicardial derived cells (EPDCs; Figure 9a). EPDCs give rise to different cardiac cell types, such as fibroblasts, SMCs, and adipocytes, and participate in the formation of coronary vessels (Smits et al., 2018). The importance of the epicardium during developmental stages mostly relies on its contribution to the de novo formation of these lineages (Figure 9a), as the lack of epicardial EMT leads to the depletion of vascular mural cells and cardiac fibroblasts (Smith et al., 2011; Acharya et al., 2012).

Following EMT, the epicardium enters a state of dormancy throughout adulthood, during which is thought to only provide a physical barrier to protect the myocardium from the pericardial fluids (Simoes and Riley, 2018). Remarkably, after cardiac injury, the epicardium reactivates developmental gene programs and quickly regenerates (Figure 9b) (Masters and Riley, 2014; Cao and Poss, 2018). It has been shown in zebrafish that, after chemical epicardial ablation, the few surviving cells proliferate and collectively migrate from the ventricular base towards its apex to repopulate the exposed myocardial surface (J. Wang et al., 2015).

a)

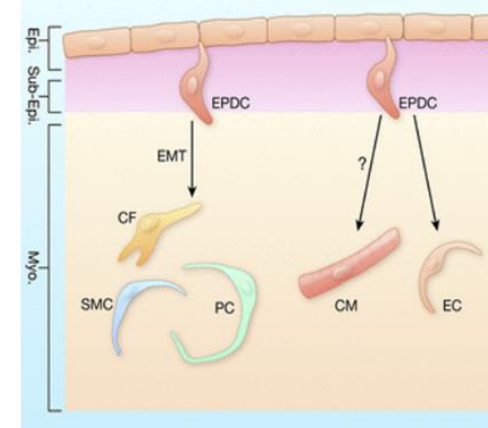

b)

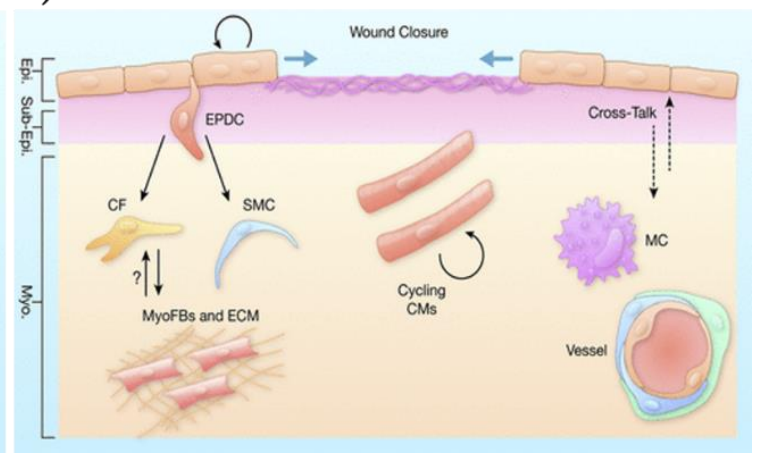

Figure 9: Epicardial processes during embryonic development (a) and cardiac regeneration (b).

a) EPDCs (epicardium-derived cells) emerge from the fetal epicardium through EMT and contribute to different lineages, such as cardiac fibroblasts (CFs), smooth muscle cells (SMCs), and pericytes (PCs). The contribution of EPDCs to endothelial cells (ECs) and cardiomyocytes (CMs) is still debated. The epicardium plays a crucial role in reciprocal paracrine signaling to stimulate CM proliferation, macrophage (MC) recruitment, and coronary vessel growth and maturation. b) The epicardium reactivates after injury and migrates initiating wound closure. It also contributes to the formation of CFs and SMCs. As well as during development, the epicardium is involved in a variety of paracrine crosstalks via the secretion of several factors. Epi, epicardium; myo, myocardium; subepi, subepicardial space. Images reprinted from Circulation Research (Quijada et al., 2020), Figures 1 and 5, with permission from Wolters Kluwer Health, Inc.

Reactivated developmental programs (such as those controlled by fetal epicardial markers Tcf21, Tbx18, Wt1) also control epicardial thickening during cardiac repair and, subsequently, a new 
EMT process (Masters and Riley, 2014; J. Wang et al., 2015; Cao and Poss, 2018). Nonetheless, the contribution of the epicardium to fibroblasts and SMCs after injury is more limited than during development, and it contributes to a small proportion of perivascular cells and fibroblasts in the forming scar (Quijada et al., 2020).

Together with its contribution to new cells during cardiac repair, the epicardium has received great attention because of its role as a reservoir of paracrine signals, with the potential to promote wound healing (Cao and Poss, 2018).

\subsubsection{The epicardium-myocardium crosstalk during development}

Together with the contribution to several cell types, another important roles for the epicardium during cardiac repair is the secretion of paracrine factors towards the myocardium. Many of these factors are conserved between development and regeneration (Figure 10). Thus, it is crucial to achieve a more detailed understanding of the epicardium-myocardium crosstalk happening during embryogenesis, which has been poorly studied thus far.

Some of the most convincing pieces of evidence pointing to a crosstalk between epicardium and myocardium rely on a common myocardial phenotype in animals presenting a reduced epicardial coverage (Kreidberg et al., 1993; Manner, 1993; Kastner et al., 1994; Sucov et al., 1994; Kwee et al., 1995; J. T. Yang et al., 1995; Moore et al., 1999; Gittenberger-de Groot et al., 2000; Pennisi et al., 2003; Manner et al., 2005; Acharya et al., 2012).

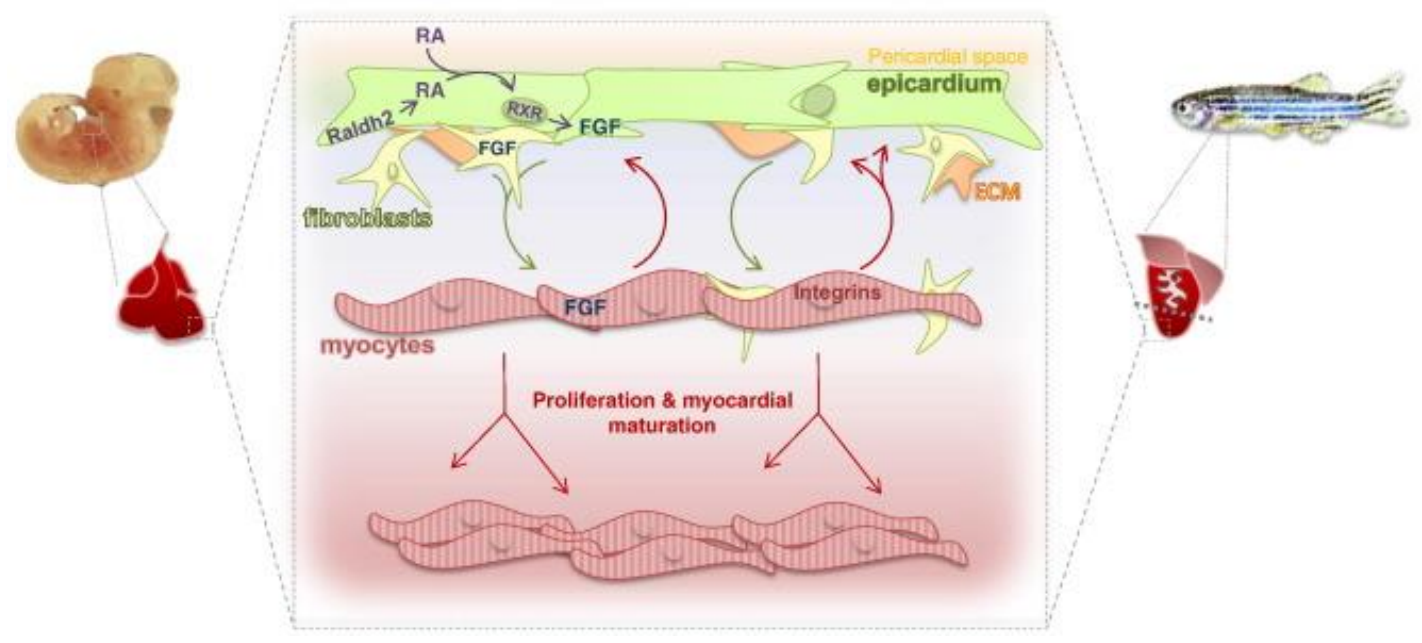

Figure 10: Epicardium-myocardium crosstalk in zebrafish and mice.

Epicardial-derived retinoic acid (RA), fibroblast growth factor (FGF), and extracellular matrix (ECM) signaling are key signals required for the development and maturation of the heart muscle. Image reprinted from Stem Cell Research (Masters and Riley, 2014), Figure 1, with permission from Elsevier.

Several experiments in chick demonstrated that the ablation of the PEO by photoablation or surgical manipulation in ovo causes reduced cardiac size and thinning of the compact layer, accompanied in some cases by ventricular bulging and formation of myocardium-pericardium 
bridges (Manner, 1993; Pennisi et al., 2003; Manner et al., 2005; Takahashi et al., 2014). Nonetheless, to date, no single causative factor is known to explain these phenotypes, and the role of the epicardium in promoting myocardial development is still debated. Some studies find the underlying reason for this phenotype is a reduction of CM proliferation (hypoplasia), while others attribute it to impaired EMT or a failed CM growth.

Similar phenotypes are obtained by the mutations of epicardial-specific genes. Two of the best studied epicardial genes are two transcription factors TCF21 and $W T 1$, which are expressed in epicardial cells from E9.5 before their delamination from the PEO. Mutations of both genes lead to midgestational (for $\mathrm{WT}^{-/}$) or perinatal $\left(\mathrm{TCF} 21^{-/}\right)$mortality, presumably due to cardiac defects. Both mutations cause reduced epicardial coverage of the heart (Moore et al., 1999; Acharya et al., 2012) and several cardiac defects, including a reduction of ventricular size. Notably, this reduction of ventricular size is observed in several other mutants of epicardial specific genes or with reduced epicardial coverage, such as retinoic acid $X$ receptors ( $R X R)$, vascular cell adhesion molecule 1 (VCAM1), Integrina4, Podoplanin mutants (Kastner et al., 1994; Sucov et al., 1994; Kwee et al., 1995; J. T. Yang et al., 1995; T. Chen et al., 2002; Merki et al., 2005; Mahtab et al., 2009). Different causes have been identified for these phenotypes. For instance, the defects in Podoplanin, Tcf21, and WT1 null mice have been attributed to a reduction of EMT, leading to a decreased number of EPDCs. Remarkably, in several cases, the observed myocardial defects begin around E11, a day before the start of the EMT process. It is, therefore, likely that other epicardial-induced defects, preceding the onset of EMT, are co-responsible for these defects. Similarly, $R X R a$ null mice and $R X R a$ epicardial-specific mutants develop normally up to E11.5, when they start exhibiting myocardial non-compaction and reduced size (Kastner et al., 1994; Sucov et al., 1994; Brade et al., 2011). The reduced cardiac size in Raldh2 null mice has been attributed to a reduction in CM proliferation (Brade et al., 2011), while another study in $R X R a$ mutant animals challenges this conclusion (Kastner et al., 1994).

\subsubsection{Molecular factors implicated in the epicardium-myocardium crosstalk}

So far, a few signaling pathways have been directly identified to be involved in this important communication, but many more are likely to be involved. The best characterized is the FGF signaling, with several of its ligands expressed by epicardial cells, including FGF-1, -2, -4, -6, 9, -16, and -18. The ligands bind to receptors located on the myocardial surface, such as FGFR1 and 2, which promote myocardial proliferation and, therefore, cardiac growth (Pennisi et al., 2003; Vega-Hernandez et al., 2011). Mice lacking FGF9 fail to form a compact myocardium, due to reduced CM cycling, and die at birth (Lavine et al., 2005). The phenotype is recapitulated by the loss of the receptors FGFR1 and FGFR2 in the myocardium (Lavine et al., 2005). On the other hand, some FGF ligands are also secreted by the myocardium, bind to FGFR2 on the epicardium to promote the recruitment of EPDCs (Masters and Riley, 2014). Another growth 
factor important for cardiac development is the insulin growth factor, IGF (P. Li et al., 2011). The expression of some of these trophic signals (i.e., Igf2 and Fgf9) from the epicardium is regulated in an autocrine manner by retinoic acid signaling (Lavine et al., 2005; Brade et al., 2011). Retinoic acid (RA) is produced by the Raldh enzymes and regulates transcription by binding to RA nuclear receptors and RXRs. In addition, RA stimulates also the hepatic secretion of erythropoietin (Epo). Epo then binds to epicardial receptors, thereby stimulating the secretion of IGF-2 (Brade et al., 2011).

Most of the observed phenotypes of the previously mentioned mutants have been ascribed to impaired CM proliferation (Pennisi et al., 2003; Lavine et al., 2005; Brade et al., 2011; P. Li et al., 2011). However, some studies do not observe impaired CM proliferation in epicardial-less embryos (Kastner et al., 1994; Takahashi et al., 2014). In addition, EPDC-derived fibroblasts cultured with adult CMs stimulate myocyte hypertrophy but not proliferation (Ieda et al., 2009), and the juxtaposition of EPDCs with CMs in culture promotes their maturation and growth (Eid et al., 1992). These and other studies challenge the view that the epicardium exclusively regulates CM cell cycle. Therefore, the role of the epicardium in facilitating hypertrophic vs hyperplastic growth still requires further investigation. 


\section{Aims of the study}

Intercellular communication comprises multiple key phenomena playing a crucial role in heart development. According to the specific location in the heart and the cell types involved, this crosstalk involves different molecular factors including signaling pathways.

In this PhD thesis, I aim at dissecting two important ways of cellular communication in the zebrafish heart, shedding light on processes not yet fully understood (Figure 11). Notably, zebrafish offers key advantages for this specific study:

1) Zebrafish is amenable to live imaging and genetic manipulation. This advantage allows the close examination of cellular and tissue behavior at single-cell resolution in vivo. Moreover, it is possible to follow the same individual over time to analyze changes in tissue morphology and cell-specific features.

2) Zebrafish can survive up to 5 days post fertilization without a functional cardiovascular system. This feature makes zebrafish an ideal model to overcome the early lethality caused by cardiac defects in mice, and study early severe cardiovascular phenotypes.

In this study I planned one overarching aim (Aim 1), achieved by the study of two different phenomena (Aim 1a, Aim 1b).

\section{Aim 1: Crosstalk between muscular and non-muscular cell types in the zebrafish heart}

Broadly, I will focus my attention on the role of non-muscle cell types in the zebrafish heart and how they can provide signaling cues necessary for muscle growth, organization, and function. The heart is considered the most important muscle, but it is the interaction of its different cell types that drives the formation of such a complex organ.

\section{Aim 1a: Endothelium-smooth muscle crosstalk in the cardiac outflow tract}

I will examine the cellular crosstalk in the zebrafish cardiac outflow tract, a poorly studied structure being placed at a crossroad of multiple cell types and signaling pathways. Combining high-resolution live-imaging, transcriptomic analyses, and cell-specific overexpression, I will show that endothelial cells play a previously unappreciated role in the development of this structure and are critical for orchestrating the formation of the smooth muscle wall. I will also provide evidence for the pivotal role that Alk5/TGF- $\beta$ signaling plays in this cellular communication, by activating a highly context-dependent downstream signaling.

\section{Aim 1b: Epicardium-myocardium crosstalk in the cardiac ventricle}

I will investigate the molecular crosstalk between the epicardium and myocardium in zebrafish larval stages before the onset of EMT. First, I aim at generating robust models of impaired epicardial coverage during embryogenesis. Combining and comparing these models, I will show 
how epicardial cues are essential to support ventricular growth by facilitating CMs volume increase. Moreover, I will characterize epicardial-derived factors, which play crucial roles in this crosstalk, strengthening previous studies in mammalian models and adding new potential candidates.

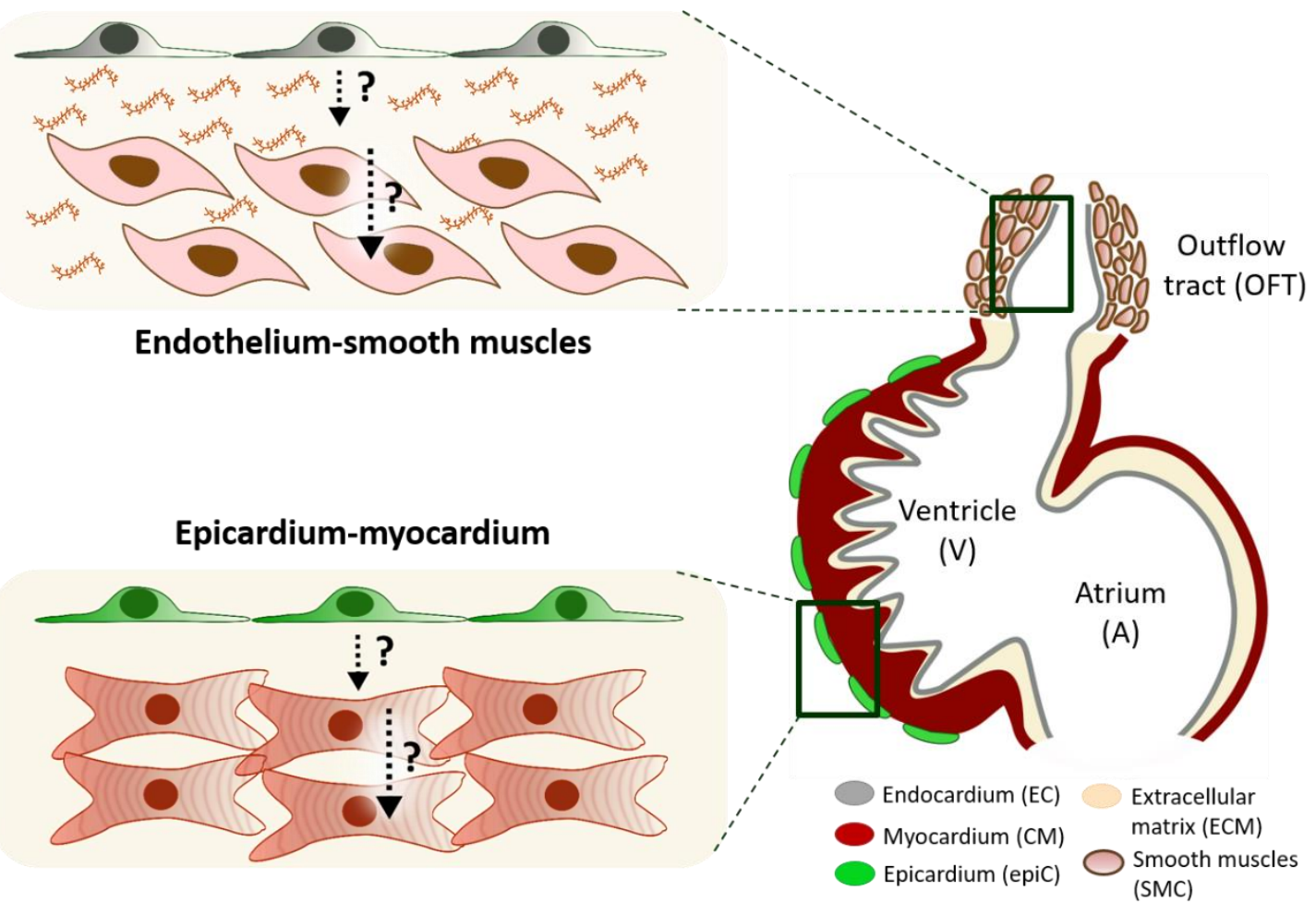

Figure 11: Aims of the study (see text) 
Materials and Methods 


\section{$3 \quad$ Materials and methods}

\subsection{Materials}

\subsubsection{Antibiotics}

Table 1: List of antibiotics with their respective working concentrations.

\begin{tabular}{|l|l|}
\hline Antibiotic & Working concentration \\
\hline Ampicillin & $100 \mu \mathrm{g} / \mathrm{mL}$ \\
\hline Kanamycin & $50 \mu \mathrm{g} / \mathrm{mL}$ \\
\hline
\end{tabular}

\subsubsection{Antibodies}

Table 2: List of antibodies used in the thesis with their respective dilution and supplier.

\begin{tabular}{|c|c|c|c|c|}
\hline Antibody & Isotype & Dilution & Supplier & Catalog \# \\
\hline $\begin{array}{l}\text { Anti-DIG-AP, Fab } \\
\text { fragments }\end{array}$ & & $1: 5,000$ & Roche & 11093274910 \\
\hline Anti-DIG POD & & $1: 500$ & Roche & 11207733910 \\
\hline Anti-Laminin & rabbit polyclonal & 1:150 & Sigma & L9393 \\
\hline anti-GFP & $\begin{array}{l}\text { Chicken } \\
\text { polyclonal }\end{array}$ & 1:400 & AvesLab & GFP-1020 \\
\hline anti-Elastin2 & rabbit polyclonal & 1:100 & (Miao et al., 2007) & \\
\hline anti-tRFP & rabbit polyclonal & $1: 200$ & Evrogen & AB233 \\
\hline $\begin{array}{l}\text { Secondaries Alexa } \\
\text { FluorTM 488-568-647 } \\
\text { IgG }(\mathrm{H}+\mathrm{L})\end{array}$ & goat polyclonal & $1: 500$ & $\begin{array}{l}\text { Thermo Fisher } \\
\text { Scientific }\end{array}$ & \\
\hline $\begin{array}{l}\text { anti-Smad3 (phospho } \\
\text { S423 + S425) }\end{array}$ & $\begin{array}{l}\text { rabbit } \\
\text { monoclonal }\end{array}$ & 1:100 & Abcam & ab52903 \\
\hline $\begin{array}{l}\text { Rabbit anti-phospho- } \\
\text { Smad1/5 } \\
\text { (Ser463/465)/ Smad9 } \\
\text { (Ser465/467) }\end{array}$ & $\begin{array}{l}\text { rabbit } \\
\text { monoclonal }\end{array}$ & 1:100 & $\begin{array}{l}\text { Cell Signaling } \\
\text { Technology }\end{array}$ & 13820 \\
\hline anti-dsRed & rabbit polyclonal & 1:200 & $\begin{array}{l}\text { Takara Bio } \\
\text { Clontech }\end{array}$ & 632496 \\
\hline $\begin{array}{l}\text { anti-Alcama/Dm- } \\
\text { Grasp }\end{array}$ & $\begin{array}{l}\text { mouse } \\
\text { monoclonal }\end{array}$ & $1: 50$ & DSHB & $\mathrm{ZN}-8$ \\
\hline
\end{tabular}




\subsubsection{Bacterial strain}

Table 3: List of bacterial strains used in the thesis with their purpose.

\begin{tabular}{|l|l|}
\hline Bacterial strain & Purpose \\
\hline DH5B & Competent cells \\
\hline
\end{tabular}

\subsubsection{Buffers and solutions}

Table 5: List of buffers and solutions along with their composition.

\begin{tabular}{|c|c|}
\hline Buffer / Solution & Composition \\
\hline Egg water & $\begin{array}{l}3 \mathrm{~g} \text { Instant Ocean } \\
0.75 \mathrm{~g} \text { Calcium sulfate } \\
10 \text { liters of distilled water }\end{array}$ \\
\hline FISH fix buffer & $\begin{array}{l}22.6 \mathrm{mM} \mathrm{NaH}_{2} \mathrm{PO}_{4} \\
77 \mathrm{mM} \mathrm{Na}_{2} \mathrm{HPO}_{4} \\
120 \mu \mathrm{M} \mathrm{CaCl}_{2} \\
117 \mathrm{mM} \text { sucrose } \\
\text { pH } 7.35\end{array}$ \\
\hline FISH fix (100 mL) & $\begin{array}{l}100 \mathrm{~mL} \text { FISH fix buffer - prewarmed at } 60^{\circ} \mathrm{C} \\
4 \mathrm{~g} \text { PFA } \\
1 \mathrm{~mL} 1 \mathrm{M} \mathrm{NaOH} \\
\text { adjusted to } \mathrm{pH} \text { to } 7.35 \text { with } \mathrm{HCl}\end{array}$ \\
\hline 1X PBS & $\begin{array}{l}1 \text { PBS tablets (Sigma) } \\
200 \mathrm{~mL} \text { distilled water }\end{array}$ \\
\hline PBST & $\begin{array}{l}0.1 \% \text { Tween-20 } \\
1 \mathrm{X} \text { PBS }\end{array}$ \\
\hline 1X PBDT & $\begin{array}{l}0.5 \% \text { Triton } \mathrm{X}-100 \\
1 \% \text { DMSO } \\
1 \% \text { BSA } \\
1 \mathrm{X} \text { PBS }\end{array}$ \\
\hline PBSTriton & $\begin{array}{l}0.1 \% \text { Triton } \mathrm{X}-100 \\
1 \mathrm{X} \text { PBS }\end{array}$ \\
\hline Hybridization mix (in situ) & $\begin{array}{l}50 \% \text { Formamide } \\
5 \mathrm{X} \mathrm{SSC} \\
0.1 \% \text { Tween } 20 \\
\text { adjusted to } \mathrm{pH} 6 \text { with } 1 \mathrm{M} \text { Citric acid } \\
\text { Heparin } 50 \mu \mathrm{g} / \mathrm{mL} \\
\text { tRNA } 500 \mu \mathrm{g} / \mathrm{mL}\end{array}$ \\
\hline 10X TBE & $\begin{array}{l}121 \mathrm{~g} \text { Tris } \\
62 \mathrm{~g} \text { Boric Acid } \\
7.4 \mathrm{~g} \text { EDTA }\end{array}$ \\
\hline
\end{tabular}




\begin{tabular}{|l|l|}
\hline \multirow{2}{*}{ 20X SSC } & 1 liter of distilled water \\
\hline & $175.3 \mathrm{~g} \mathrm{NaCl}$ \\
& $88.2 \mathrm{~g}$ Sodium citrate \\
& $800 \mathrm{~mL}$ of distilled water \\
& Adjusted to $\mathrm{pH} 7$ \\
\hline \multirow{3}{*}{ Blocking buffer (in situ) } & $2 \mathrm{mg} / \mathrm{mL} \mathrm{BSA}$ \\
& $2 \%$ sheep serum \\
& $0.1 \%$ PBST \\
\hline Alkaline Tris buffer & $100 \mathrm{mM}$ Tris-HCl $\mathrm{pH} 9.5$ \\
& $100 \mathrm{mM}$ NaCl \\
& $0.1 \%$ Tween 20 \\
\hline \multirow{3}{*}{ Richardson solution } & distilled water \\
\hline & Azure II \\
& Methylene blue \\
& di-Sodium tetraborate (Borax, water free) \\
& Distilled water \\
\hline
\end{tabular}

\subsubsection{Centrifuges}

Table 4: List of centrifuges.

\begin{tabular}{|l|l|}
\hline Centrifuge & Supplier \\
\hline Centrifuge (1.5-2 $\mathrm{mL}$ tubes) $\mathbf{5 4 1 8}$ & Eppendorf \\
\hline Centrifuge (1.5-2 $\mathrm{mL}$ tubes) $\mathbf{5 4 1 5}$ & Eppendorf \\
\hline Centrifuge (15 $\mathrm{mL}, \mathbf{5 0} \mathrm{mL}$ tubes) $\mathbf{5 8 1 0 R}$ & Eppendorf \\
\hline
\end{tabular}

\subsubsection{Chemicals}

Table 6: List of chemicals used in the thesis with their respective supplier.

\begin{tabular}{|c|c|c|}
\hline Chemical & Supplier & Catalog \# \\
\hline Bovine serum albumin (BSA) & Sigma & A2153 \\
\hline Chloroform & Merck & 102445 \\
\hline DNA ladder (100 bp) & Thermo Scientific & SM0241 \\
\hline DNA ladder (1 kb) & Thermo Scientific & SM0311 \\
\hline Ethanol & Roth & K928.3 \\
\hline Methanol & Roth & 4627.5 \\
\hline Paraformaldehyde (PFA) & Sigma & P6148 \\
\hline Gel loading dye & Thermo Scientific & R0611 \\
\hline Isopropanol & Roth & 6752,4 \\
\hline Methylene blue & Sigma & M9140 \\
\hline
\end{tabular}




\begin{tabular}{|c|c|c|}
\hline $\begin{array}{l}\text { Tricaine (ethyl-m-aminobenzoate } \\
\text { methanesulfonate) }\end{array}$ & Sigma & $886-86-2$ \\
\hline Phosphate-buffered saline (PBS) tablets & Sigma & P4417 \\
\hline Dimethylsulfoxide (DMSO) & Sigma & D4540 \\
\hline Goat serum & Sigma & \\
\hline Fetal bovine serum (FBS) & Biochrom & S0615 \\
\hline Tris & Sigma & 5429.2 \\
\hline Tris hydrochloride (Tris-HCl) & Sigma & RES3098T-B701X \\
\hline Tween-20 & Sigma & P1379 \\
\hline CutSmart buffer & NEB & B7204S \\
\hline T4 ligase buffer & Takara & SD0267 \\
\hline Agarose, low gelling temperature & Sigma & A9414 \\
\hline Trizol & Ambion & 15596018 \\
\hline Pronase & Roche & 10165921001 \\
\hline Agarose & Peqlab & $35-1020$ \\
\hline SYBR safe & Invitrogen & S33102 \\
\hline 1-Phenyl-2-thiourea (PTU) & Sigma & P7629 \\
\hline Calcium chloride $\left(\mathrm{CaCl}_{2}\right)$ & Merck & $10035-04-8$ \\
\hline Triton X-100 & Sigma & RES3103T-A101X \\
\hline DAPT & Merck & 565770 \\
\hline Hydrochloridic acid (HCl) & Sigma & H1758 \\
\hline Sodium hydroxide $(\mathrm{NaOH})$ & Sigma & 221465 \\
\hline Phenol red & Sigma & P0290 \\
\hline Proteinase $\mathrm{K}$ & Roche & 1092766 \\
\hline Sucrose & Sigma & S0389 \\
\hline Hydrogen peroxide $\left(\mathrm{H}_{2} \mathrm{O}_{2}\right)$ & Sigma & $7722-84-1$ \\
\hline 5-ethynyl-2'-deoxyuridine (EdU) & ThermoFisher & A10044 \\
\hline Phalloidin 568 & Invitrogen & A12380 \\
\hline Sodium citrate & Sigma & $18996-35-5$ \\
\hline DIG RNA labeling mix (Sp6/T7) & Roche & 11277073910 \\
\hline Citric acid & Sigma & 27109 \\
\hline Heparin & Sigma & 136098-10-7 \\
\hline Sheep serum & Sigma & ABIN925265 \\
\hline tRNA & Sigma & 9014-25-9 \\
\hline BM Purple AP substrate & Roche & 11442074001 \\
\hline 2-Dodecenylsuccinic acid anhydride (DDSA) & Serva & 20755.02 \\
\hline $\begin{array}{l}\text { 2,4,6-Tris(dimethylaminomethyl)phenol } \\
\text { (DMP) }\end{array}$ & Serva & 36975.01 \\
\hline
\end{tabular}




\begin{tabular}{|c|c|c|}
\hline Acetone, anhydrous & VWR & 83683.290 \\
\hline Azure II & Carl Roth & 7640.1 \\
\hline Cacodylic acid-Na-salt- $3 \mathrm{H}_{2} \mathrm{O}$ & Serva & 15540.02 \\
\hline di-Sodium tetraborate (Borax, water free) & Carl Roth & 4403.1 \\
\hline Glutaraldehyde & Carl Roth & 4157.1 \\
\hline Glycid ether 100 & Serva & 21045.02 \\
\hline Methylnadic anhydride (MNA) & Serva & 29452.03 \\
\hline Osmium tetroxide, $4 \%$ aqueous solution & Science Services & E19190 \\
\hline Paraformaldehyde, granulated & Carl Roth & 0335.3 \\
\hline Uranyl acetate $\cdot 2 \mathrm{H}_{2} \mathrm{O}$ & Serva & 77870.01 \\
\hline E-616452 & Cayman Chemical & 14794 \\
\hline SIS3 & Calbiochem/Merck & 566405 \\
\hline SU-5402 & Sigma & $215543-92-3$ \\
\hline SU-5416 & Sigma & $204005-46-9$ \\
\hline EdU & Thermo Fisher & A10044 \\
\hline FITC-dextran $2000 \mathrm{kDa}$ & Sigma & 52471 \\
\hline LysoTracker Deep Red & ThermoFisher & L12492 \\
\hline Metronidazole & Sigma & M1547 \\
\hline DAF-FM & Sigma & $254109-22-3$ \\
\hline
\end{tabular}

\subsubsection{Enzymes}

Table 7: List of enzymes used in the thesis with their respective supplier.

\begin{tabular}{|l|l|}
\hline Enzyme & NEB \\
\hline DpnI & NEB \\
\hline SalI & NEB \\
\hline BamHI-HF & NEB \\
\hline XbaI & NEB \\
\hline EcoRI-HF & NEB \\
\hline XhoI & NEB \\
\hline AvrII & NEB \\
\hline AscI & NEB \\
\hline NheI & NEB \\
\hline ClaI & NEB \\
\hline HindIII & NEB \\
\hline NotI & Kapa Biosystem \\
\hline KAPA 2G fast DNA polymerase & Takara \\
\hline PrimeSTAR max polymerase & Thermo Scientific \\
\hline DyNAmo ColorFlash SYBR Green qPCR Mix & \\
\hline
\end{tabular}




\begin{tabular}{|l|l|}
\hline DNase I & Qiagen \\
\hline T7 RNA polymerase & Promega \\
\hline RNasin ribonuclease inhibitor & Promega \\
\hline
\end{tabular}

\subsubsection{Growth media}

Table 8: List of growth media used in the thesis along with their composition.

\begin{tabular}{|l|l|}
\hline Growth medium & Composition \\
\hline Gibco TM DMEM & Thermo Fisher Scientific \\
\hline LB agar & Roth \\
\hline LB medium & Roth \\
\hline
\end{tabular}

\subsubsection{Commercial kits}

Table 9: List of kits used in the thesis with their respective supplier.

\begin{tabular}{|c|c|c|}
\hline Kit & Supplier & Catalog \# \\
\hline RNA clean and concentrator & Zymo Research & R1016 \\
\hline mMESSAGE mMACHINE kit (T3) & Ambion & AM1348 \\
\hline mMESSAGE mMACHINE kit (T7) & Ambion & AM1344 \\
\hline MEGAshortscript T7 kit & Ambion & AM1354 \\
\hline GeneJET PCR purification kit & Thermo Scientific & K0701 \\
\hline GeneJET gel extraction kit & Thermo Scientific & K0691 \\
\hline GeneJET plasmid miniprep kit & Thermo Scientific & K0502 \\
\hline pGEM-T-easy vector kit & Promega & A1360 \\
\hline miRNeasy micro kit & Qiagen & 217084 \\
\hline In situ cell death detection kit & Roche & 11684795910 \\
\hline Click-iTTM EdU Alexa Fluor $^{\text {TM }} 647$ & Invitrogen & C10340 \\
\hline DIG RNA labelling kit & Roche & 11277073910 \\
\hline Maxima First Strand cDNA kit & Thermo Scientific & K1641 \\
\hline VAHTS Stranded mRNA-seq Library & Vazyme & NR612 \\
\hline Dual-Luciferase® Reporter Assay & Promega & E1910 \\
\hline In-Fusion ${ }^{\circledR}$ HD Cloning Plus & Takara Bio & 638910 \\
\hline
\end{tabular}

\subsubsection{Laboratory supplies}

Table 10: List of laboratory supplies used in the thesis with their respective supplier.

\begin{tabular}{|l|l|}
\hline Laboratory supply & Supplier \\
\hline Bacterial culture tube & Sarstedt \\
\hline Latex gloves & Roth \\
\hline Nitrile gloves & VWR \\
\hline Beakers & VWR \\
\hline
\end{tabular}




\begin{tabular}{|c|c|}
\hline Centrifuge tubes $(1.5 \mathrm{~mL}, 2 \mathrm{~mL})$ & Sarstedt \\
\hline Centrifuge tubes $(5 \mathrm{~mL})$ & Eppendorf \\
\hline Falcons $(15 \mathrm{~mL}, 50 \mathrm{~mL})$ & Greiner bio-one \\
\hline Glass bottom dish & MatTek \\
\hline Microloader pipette tips & Eppendorf \\
\hline PCR tubes $(200 \mu \mathrm{L})$ & Sarstedt \\
\hline Scalpel & Braun \\
\hline Petri dish $(90 \mathrm{~mm}, 60 \mathrm{~mm}, 35 \mathrm{~mm})$ & Grainer bio-one \\
\hline Forceps & Dumont \\
\hline 4-well Chambered Coverglass & ThermoFisher Scientific \\
\hline Glass bottles $(50 \mathrm{~mL}-1000 \mathrm{~mL})$ & Duran \\
\hline Laboratory film & Parafilm \\
\hline Pipettes $(2 \mu \mathrm{L}, 20 \mu \mathrm{L}, 200 \mu \mathrm{L}, 1000 \mu \mathrm{L})$ & Gilson \\
\hline Pipetboy & Integra \\
\hline Pipette filter tips & Grainer bio-one \\
\hline Pipette tips & Grainer bio-one \\
\hline Insulin syringes & Terumo \\
\hline
\end{tabular}

\subsubsection{Microscopes}

Table 11: List of microscopes used in the thesis with their respective supplier.

\begin{tabular}{|l|l|}
\hline Microscope & Supplier \\
\hline Stereomicroscope Stemi 2000 & Zeiss \\
\hline Stereomicroscope SMZ18 & Nikon \\
\hline Stereomicroscope SMZ25 & Nikon \\
\hline Confocal microscope LSM700 & Zeiss \\
\hline Confocal microscope LSM800 Observer & Zeiss \\
\hline Confocal microscope LSM800 Examiner & Zeiss \\
\hline Confocal microscope LSM880 & Zeiss \\
\hline Lightsheet Z.1 microscope & Zeiss \\
\hline Spinning Disk microscope & Zeiss \\
\hline Jeol JEM-1400 Plus & Jeol \\
\hline
\end{tabular}

\subsubsection{Laboratory equipment}

Table 12: List of miscellaneous laboratory equipment

\begin{tabular}{|l|l|}
\hline Equipment & Supplier \\
\hline Nanodrop 2000c & Thermo Scientific \\
\hline Injection micromanipulator & World precision instruments \\
\hline
\end{tabular}




\begin{tabular}{|l|l|}
\hline PCR mastercycler Pro & Eppendorf \\
\hline CFX connect real time PCR & Bio Rad \\
\hline Bullet Blender® Homogenizer & Scientific instrument services \\
\hline NextSeq500 instrument & Illumina \\
\hline Eco Real-Time PCR System & Illumina \\
\hline LabChip Gx Touch 24 & Perkin Elmer \\
\hline Dark reader transilluminator & Clare chemical \\
\hline Gel Doc EZ system & Bio Rad \\
\hline Electrophoresis power supply & Bio Rad \\
\hline Microscale & Novex \\
\hline Weighing balance & Sartorius \\
\hline Micropipette puller P-1000 & Sutter instruments \\
\hline Bacterial incubator shaker & Infors HAT \\
\hline Bacterial incubator & Heraeus \\
\hline Heating block & VWR \\
\hline Microwave & Bosh \\
\hline Zebrafish aqua culture system & Tecniplast \\
\hline Zebrafish incubator & Binder \\
\hline Slot grid (Copper) 2x1mm & Plano \\
\hline Ultracut E microtome & Reichert-Jung \\
\hline BD FACSAriaTM III Cell Sorter & BD Biosciences \\
\hline
\end{tabular}

\subsubsection{Oligonucleotides}

Table 13: List of oligonucleotides used in the thesis with their sequence and purpose.

\begin{tabular}{|c|c|c|}
\hline Oligonucleotide & Sequence $5^{\prime}-3^{\prime}$ & Purpose \\
\hline alk $5 b \mathrm{fw}$ & GCACCACACTCAAAGACCTG & RT-qPCR \\
\hline alk5b rV & CCCTTACCGATGCTCTCCTG & RT-qPCR \\
\hline alk5a fw & CACCCATTCCTTGCTGATGG & RT-qPCR \\
\hline alk5a rv & ССТTCCСАATGCTCTCTTGG & RT-qPCR \\
\hline$r p 113 \mathrm{fw}$ & AATTGTGGTGGTGAGGTG & RT-qPCR \\
\hline rp113 IV & GGTTGGTGTTCATTCTCTTG & RT-qPCR \\
\hline$f b \ln 5 \mathrm{fw}$ & CTGTGAAGACCTCAATGAATGTG & RT-qPCR \\
\hline fbln5 rv & CCGACAAGCAGGATTCTCAG & RT-qPCR \\
\hline sema3d fw & GTGCTCCAGATACATCCCAG & RT-qPCR \\
\hline sema3d $\mathrm{rV}$ & ACATTCCTCCCTAACACATCCTC & RT-qPCR \\
\hline efemp2b fw & TCATGCAATGACATCAACGA & RT-qPCR \\
\hline efemp2brv & GTAGCCTTCTGGACAAACAC & RT-qPCR \\
\hline
\end{tabular}




\begin{tabular}{|c|c|c|}
\hline T7 & TAATACGACTCACTATAGGG & Sequencing \\
\hline M13 foward & GTAAAACGACGGCCAGTG & Sequencing \\
\hline alk5b HA1 GFP fw & $\begin{array}{l}\text { ACTGGAGTCCAGCAGGAGAACAGAAGAGGAGC } \\
\text { GGGATTATCTCCAGGAGGACCATGGTGAGCAA } \\
\text { GGGCGAGGAG }\end{array}$ & \multirow{2}{*}{$\begin{array}{l}\text { To insert } \\
\text { eGFP_Kan in } \\
\text { CH1073-59L16 } \\
\text { clone }\end{array}$} \\
\hline alk5b HA2 Kan rv & $\begin{array}{l}\text { ACCAGTCGACACGGCACTGCCTCCATCATCAT } \\
\text { CATCTTCATCATCATCTTTTCCAGAAGTAGTG } \\
\text { AGGAG }\end{array}$ & \\
\hline Fosmid iTol2_eye fw & $\begin{array}{l}\text { TTCTCTGTTTTTGTCCGTGGAATGAACAATGG } \\
\text { AAGTCCGAGCTCATCGCTCCCTGCTCGAGCCG } \\
\text { GGCCCAAGTG }\end{array}$ & \multirow{2}{*}{$\begin{array}{l}\text { To insert } \\
\text { cryaa:CFP_iTol2 in } \\
\text { the CH1073-59L16 } \\
\text { clone }\end{array}$} \\
\hline Fosmid iTol2_eye rv & $\begin{array}{l}\text { AGCCCCGACACCCGCCAACACCCGCTGACGCG } \\
\text { AACCCCTTGCGGCCGCATGAAACAGCTATGAC } \\
\text { CATGTAA }\end{array}$ & \\
\hline alk5b ATG fw & GGATGAAGATGATGATGAAGATG & \multirow{4}{*}{$\begin{array}{l}\text { To generate the } \\
\text { fli1 a:tofbr1b-P2A- } \\
m \text { Scarlet-Hsa.HRAS }\end{array}$} \\
\hline alk $5 b$ noSTOP rv & GGTACCCCGACACGGCAC & \\
\hline fli1a fw & TTGGAGATCTCATCTTTGAC & \\
\hline fli1a rev & GGTGGCGCTAGCTTCGCGTCTGAATTAATTCC & \\
\hline tgfbr1a sgRNA exon1 & GGATGATCTTTACCCCC & $\begin{array}{l}\text { sgRNA for } \\
\text { CRISPR/Cas9 } \\
\text { mutagenesis }\end{array}$ \\
\hline $\operatorname{tgfbr} 1 b$ sgRNA exon 4 & GAGGAGCGCTCCTGGTTC & $\begin{array}{l}\text { sgRNA for } \\
\text { CRISPR/Cas9 } \\
\text { mutagenesis }\end{array}$ \\
\hline alk5a HRM fw & CTTCTGGACAGACCGTGACA & \multirow{2}{*}{$\begin{array}{l}\text { HRM based } \\
\text { genotyping primers } \\
\text { for tgfbr } 1 a^{b n s} 329\end{array}$} \\
\hline alk5a HRM rev & GAAGGAGCGCACTGGAAAG & \\
\hline alk5b HRM fw & CGCTGGAGAGGAGAGGAGG & \multirow{2}{*}{$\begin{array}{l}\text { HRM based } \\
\text { genotyping primers } \\
\text { for tofbr } 1 b^{b n s 225}\end{array}$} \\
\hline alk $5 b$ HRM rev & GTCTCAGCATGACGGTCTGG & \\
\hline alk $5 b$ rescue $\mathrm{fw}$ & AGAGGAGAGGAGGTGGCG & \multirow{2}{*}{$\begin{array}{l}\text { HRM based } \\
\text { genotyping primers } \\
\text { for tofbr } 1 b^{\text {bns } 225} \text { with } \\
\text { Tg(fli1 a:tgfbr1b-P2A- } \\
\text { mScarlet.Hsa.HRAS)b } \\
{ }_{n: 241}\end{array}$} \\
\hline alk $5 b$ rescue rev & CCCATGATGCCCCAGTGC & \\
\hline fbln5 ATG fw & ATGTTTGTTGAACTACGTGGC & \multirow{2}{*}{$\begin{array}{l}\text { To generate } \\
\text { fli1a:fbln5,EGFP } \\
\text { and pCS2+, fbln5 }\end{array}$} \\
\hline fbln5 STOP rv & TCAGAATGGATGTTCGGAGAC & \\
\hline
\end{tabular}




\begin{tabular}{|c|c|c|}
\hline alk $5 b$ ISH fw & TCATGAGCACGGTTCTCTGT & \multirow{2}{*}{$\begin{array}{l}\text { Generation of alk. } 5 b \\
\text { ISH probe }\end{array}$} \\
\hline alk $5 b$ ISH T7 rv & $\begin{array}{l}\text { GTAATACGACTCACTATAGGATCCCCAATGAG } \\
\text { TCGGTGTT }\end{array}$ & \\
\hline$f b \ln 5 \mathrm{ISH}$ fw & TGTTCTCACAGGTTCTTGCC & \multirow{2}{*}{$\begin{array}{l}\text { Generation of } f b \ln 5 \\
\text { ISH probe }\end{array}$} \\
\hline fbln5 ISH T7 rv & $\begin{array}{l}\text { TAATACGACTCACTATAGGGCAGGGCTACAGG } \\
\text { AACAGGAA }\end{array}$ & \\
\hline$t c f 21$ sgRNA exon1 & CGCAGCTAACGCGCGCGAGA & $\begin{array}{l}\text { sgRNA for } \\
\text { CRISPR/Cas9 }\end{array}$ \\
\hline wt1a sgRNA1 & CAACTGGACAGCTTGGCCTA & $\begin{array}{l}\text { sgRNA for } \\
\text { CRISPR/Cas9 }\end{array}$ \\
\hline wt1a sgRNA2 & AAAGGCGTCTAATAGACAAC & $\begin{array}{l}\text { sgRNA for } \\
\text { CRISPR/Cas9 }\end{array}$ \\
\hline wt1a sgRNA3 & GGCAGTGCCACTCTTGCCAG & $\begin{array}{l}\text { sgRNA for } \\
\text { CRISPR/Cas9 }\end{array}$ \\
\hline tcf $21 \mathrm{HRM}$ fw & GCAAGCAGGTCCAGAGGA & \multirow{2}{*}{$\begin{array}{l}\text { HRM based } \\
\text { genotyping primers } \\
\text { for } \operatorname{tcf} 21^{\text {bns } 427}\end{array}$} \\
\hline tcf21 HRM rv & ACGGTAACGTCGTCTTCAGC & \\
\hline$t c f 21$ geno fw & TGTCTCCAGCCAACATGTCCA & \multirow{2}{*}{$\begin{array}{l}\text { PCR based } \\
\text { genotyping primers } \\
\text { for } \operatorname{tcf} 21 \text { bns } 427 \text { carrying } \\
\operatorname{TgB} A C(t c f 21: n l s- \\
\text { EGFP) }\end{array}$} \\
\hline tcf21 geno rev & GCGCATCCTCGCCCTCTCG & \\
\hline wt1a 5' F1 & TGTGAAATGAATGACACATCAAG & \multirow{3}{*}{$\begin{array}{l}\text { PCR based } \\
\text { genotyping primers } \\
\text { for } w t 1 a^{b n s} 428\end{array}$} \\
\hline wt1a 3' R1 & GCTGATCATCTCTGCGTTTG & \\
\hline wt1a 3' int1 F2 & CAATTGAAAAACTTTAAAAATCAGCA & \\
\hline tcf21 ISH fw & CGCATGACACGTTTCCACAT & \multirow{2}{*}{$\begin{array}{l}\text { Generation of } t c f 21 \\
\text { ISH probe }\end{array}$} \\
\hline tcf21 ISH T7rv & $\begin{array}{l}\text { GGTAATACGACTCACTATAGGTGACATGACAC } \\
\text { TCGGCGT }\end{array}$ & \\
\hline wt1a ISH fw & AAATGGCGTCACAGTTGGAG & \multirow{2}{*}{$\begin{array}{l}\text { Generation of } w t 1 a \\
\text { ISH probe }\end{array}$} \\
\hline wt1a ISH T7rv & $\begin{array}{l}\text { GGTAATACGACTCACTATAGGTGTAATCAATC } \\
\text { GACCTGCAGTG }\end{array}$ & \\
\hline
\end{tabular}




\subsubsection{Plasmids}

Table 14: List of plasmids used, along with their antibiotic resistance and source.

\begin{tabular}{|l|l|l|}
\hline Plasmid & Resistance & \multicolumn{1}{l|}{ Aource } \\
\hline pT3TS-nlsCas9nls vector & Ampicillin & Addgene \\
\hline pGEM-T & Ampicillin & Promega \\
\hline pTo12 fli1a:tgfbr1b-P2A-mScarlet-hRAS & Ampicillin & Addgene \\
\hline pCMV-Tol2 & Ampicillin & (Uribe et al., 2018) \\
\hline pTo12 myl7:mScarlet & Ampicillin & (Boezio et al., 2020) \\
\hline pTol2 fli1a:fbln5,EGFP & & \\
\hline
\end{tabular}

\subsubsection{Softwares}

Table 15: List of software used in the thesis with their respective purpose.

\begin{tabular}{|l|l|}
\hline Software & Purpose \\
\hline SnapGene (Insightful Science) & Sequence analysis, primer design \\
\hline Inkscape & Figures formatting \\
\hline Prism (GraphPad Software) & Data analysis \\
\hline ImageJ & Image processing \\
\hline Imaris (Bitplane) & Image processing \\
\hline ZEN Blue and ZEN Black (Zeiss) & Image processing \\
\hline Microsoft Office & Writing, data analysis, image formatting \\
\hline Perl Primer & qPCR primer design \\
\hline Primer 3 & Primer design \\
\hline Ensemb1 & Genomic Sequences \\
\hline FastQC & Bioinformatics analysis \\
\hline Gitools & Bioinformatics analysis \\
\hline
\end{tabular}

\subsubsection{Zebrafish lines}

Table 17: List of zebrafish lines used in the thesis along with the publications where they were first described.

\begin{tabular}{|c|c|c|}
\hline Line & Details & Publication \\
\hline $\mathbf{A B}$ & Wild type & \\
\hline$T g(k d r l: E G F P) s 843$ & Endothelial reporter & (Jin et al., 2005) \\
\hline $\operatorname{Tg}(-5.1$ myl7:DsRed2-NLS)f2 & Nuclear CM reporter & (Rottbauer et al., 2002) \\
\hline$T g(m y 17: a c t n 3 b-E G F P) s d 10$ & Sarcomere reporter & (Y. F. Lin et al., 2012) \\
\hline Tg(myl7:EGFP-Hsa.HRAS)s883Tg & $\begin{array}{l}\text { CM membrane } \\
\text { reporter }\end{array}$ & (D'Amico et al., 2007) \\
\hline
\end{tabular}




\begin{tabular}{|c|c|c|}
\hline $\operatorname{TgBAC}(\operatorname{tcf} 21: D s R e d 2) p d 37$ & Epicardial reporter & $\begin{array}{l}\text { (Kikuchi, Gupta, et al., } \\
\text { 2011) }\end{array}$ \\
\hline TgBAC(tcf21:mCherry-NTR)pd108 & Epicardial reporter & (J. Wang et al., 2015) \\
\hline$T g B A C(t c f 21: N L S-E G F P) p d 41$ & Epicardial reporter & (J. Wang et al., 2011) \\
\hline $\operatorname{Tg}(m y 17: B F P-C A A X) b n s 193$ & $\begin{array}{l}\text { CM membrane } \\
\text { reporter }\end{array}$ & (Guerra et al., 2018) \\
\hline$T g B A C($ vegfaa:EGFP)pd260 & Vegfaa reporter & (Karra et al., 2018) \\
\hline $\operatorname{Tg}(m y 17: m C h e r y-C A A X)$ & $\begin{array}{l}\text { CM membrane } \\
\text { reporter }\end{array}$ & (Uribe et al., 2018) \\
\hline Tg(kdrl:TagBFP)mu293 & Endothelial reporter & (Matsuoka et al., 2016) \\
\hline Tg(kdrl:dsRed2)pd27 & Endothelial reporter & $\begin{array}{l}\text { (Kikuchi, Holdway, et al., } \\
\text { 2011) }\end{array}$ \\
\hline Tg(fli1a:Gaf4ff)ubs4 & Endothelial driver & (Zygmunt et al., 2011) \\
\hline $\operatorname{Tg}($ UAS:Kaede)rk8 & $\begin{array}{l}\text { UAS reporter driving } \\
\text { Kaede }\end{array}$ & (Herwig et al., 2011) \\
\hline$T g B A C(p d g f r b: E G F P) n c v 22$ & Mural cell reporter & (Ando et al., 2016) \\
\hline Tg(kdrl:NLS-mCherry)is 4 & $\begin{array}{l}\text { Endothelial nuclear } \\
\text { reporter }\end{array}$ & $\begin{array}{l}\text { (Y. Wang, Kaiser, et al., } \\
\text { 2010) }\end{array}$ \\
\hline Tg(12xSBE:EGFP)ia16 & Smad3 reporterr & (Casari et al., 2014) \\
\hline TgBAC(tgfbr1b:EGFP,cryaa:CFP)bns330 & alk:5b/tofbr1b reporter & (Boezio et al., 2020) \\
\hline $\begin{array}{l}\text { Tg(fli1a:tgfbr1b-P2A-mScarlet- } \\
\text { Hsa.HRAS)bns } 421\end{array}$ & $\begin{array}{l}\text { EC-specific alk } 5 b \\
\text { overexpression }\end{array}$ & (Boezio et al., 2020) \\
\hline $\operatorname{tgfbr1a^{\text {bns329}}}$ & alk. 5 a mutants & (Boezio et al., 2020) \\
\hline $\operatorname{tgfbr} 1 b^{\text {bns } 225}$ & alk. $5 \mathrm{~b}$ mutants & (Boezio et al., 2020) \\
\hline tcf $21^{\text {bns } 427}$ & tcf21 mutants & This study \\
\hline$w t 1 a^{\text {bns } 428}$ & $w t 1 a$ mutants & This study \\
\hline
\end{tabular}




\subsection{Methods}

\subsubsection{Zebrafish maintenance and breeding}

Zebrafish husbandry was performed under standard conditions (Westerfield, 2000). Adult zebrafish were kept in an aquaculture system (Tecniplast) with a water temperature of $28^{\circ} \mathrm{C}$ and room temperature of $29^{\circ} \mathrm{C}$. The water $\mathrm{pH}$ is kept at $6.8-7.5$ and the 14 hours- 10 hours lightdark cycles were maintained in the room to mimic day-night cycles. Animal housing and experiments were approved by the MPG animal committee in concordance with the European regulations.

Zebrafish male and female adults were transferred during the afternoon into a mating tank equipped with a transparent net, separating the fish from the bottom of the tank were eggs will be deposited, and a transparent divider to separate males and females, to prevent mating during the night. The next morning at the desired time, dividers were removed and fertilized eggs could be collected from the bottom of the tank. Embryos were transferred in $9 \mathrm{~cm}$ petri dishes filled with egg-water, at a density of $50 \mathrm{eggs} / \mathrm{dish}$, and placed in an incubator at $28^{\circ} \mathrm{C}$. The same evening, dead or unfertilized embryos were removed under a stereomicroscope based on their morphology.

At $24 \mathrm{hpf}, 0.0045 \%$ PTU was added in the medium of to prevent the pigmentation. When needed, the chorions were removed manually with forceps or by adding $300 \mu$ l of pronase (10 $\mathrm{mg} / \mathrm{ml}$ ) to $50 \mathrm{ml} \mathrm{egg} \mathrm{water.}$

\subsubsection{RNA and DNA microinjections into once-cell stage zebrafish embryos}

In order to inject plasmids, mRNA, or reagents for CRISPR/Cas9, the solutions at the desired concentrations were loaded into injection needles. The needles were obtained from borosilicate glass capillaries heated and pulled to obtain very fine tips. The injection needles were fixed onto a micromanipulator and the tip was opened with forceps. Pressure conditions of the micromanipulator were calibrated to allow a small droplet to come out of the needle and, when necessary, the droplet volume was calibrated. Embryos were collected after 15-20 minutes from spawning, to allow for their fertilization to take place, and transferred to a petri dish with eggwater. They were, then, aligned on a $2 \%$ agarose injection plate and injected at one-cell stage. For plasmid injections, the injection solution contained Tol2 transposase mRNA (25 pg per embryo) and plasmid DNA (5-25 pg per embryo). The amount of DNA injected was standardized using serial dilution methods, in order to reach good fluorescence visualization while preventing toxicity. For $f b \ln 5$ RNA injections, $f b \ln 5$ wild-type cDNA sequence was transcribed in vitro and $150 \mathrm{pg}$ RNA/embryo were injected into one-cell stage embryos. 


\subsubsection{DNA and RNA isolation from zebrafish embryos}

DNA and RNA were extracted from single embryos using TRIzol reagent and homogenized using a bullet blender (Next Advance). Chloroform was then added and the samples centrifuged 5 minutes at maximum speed. The upper aqueous phase, containing RNA, was collected, mixed with 1 volume isopropanol and stored at $-80^{\circ} \mathrm{C}$. DNA was then precipitated from the organic phase after the addition of $70 \%$ ethanol. The pellet was then treated with proteinase $\mathrm{K}$, to allow for protein degradation and genotyping. After the genotyping, RNA from 5-10 embryos of the same genotype was pooled and extracted by precipitation with the RNA Clean and Concentrator Kit (Zymo Research), following manufacturer's instructions, and then quantified using NanoDrop. Only samples with 260/280 ratios above 1.95 were used for further analyses. RNA was stored at $-80^{\circ} \mathrm{C}$.

\subsubsection{Embryonic heart isolation and RNA extraction}

Hearts were dissected from 50,56, 72, 80, 96 hpf animals, depending on the experiment, carrying a transgene allowing for the visualization of the heart under a fluorescence microscope (Tg(kdrl:EGFP) or $\operatorname{Tg}(m y l 7: E G F P-h R A S))$. Embryos or larvae were transferred to a Petri dish containing DMEM $+10 \%$ FBS and anesthetized using $0.1 \%$ tricaine. The hearts were manually dissected using forceps or insulin needles under a fluorescence stereomicroscope, paying attention not to damage the chambers and to remove the excessive surrounding tissues. According to the experiment, 9 (for RT-qPCR), 20 (for whole-heart bulk RNAseq) or $>100$ (for sorted CMs or epicardial cells RNA-seq and for scRNAseq) were pooled per replicate, transferred to $1.5 \mathrm{ml}$ tubes on ice. The samples were then processed for digestion preceding FACS sorting (3.2.7) or total RNA extraction. The tubes were spinned and the remaining medium solution was removed and substituted by $70 \mu \mathrm{l}$ of Trizol. Following Trizol/chloroform extraction, the aqueous phase containing RNA was purified using the miRNeasy micro kit, combined with on-column DNase digestion. When necessary, hearts were dissected and the body of the larva was genotyped.

\subsection{5 cDNA synthesis and real-time quantitative PCR (RT-qPCR)}

500 ng RNA was used to synthesize cDNA using the Maxima First Strand cDNA kit, following manufacturer's instructions and the following reaction conditions:

\begin{tabular}{|l|l|}
\hline Temperature & Time \\
\hline $\mathbf{2 5}^{\circ} \mathbf{C}$ & 10 minutes \\
\hline $\mathbf{5 0}^{\circ} \mathbf{C}$ & 15 minutes \\
\hline $\mathbf{8 5}^{\circ} \mathbf{C}$ & 5 minutes \\
\hline
\end{tabular}


The cDNA was then diluted 1:5 in nuclease-free water. DyNAmo ColorFlash SYBR Green qPCR Mix was used and RT-PCR was performed on a CFX connect Real-time System (Bio Rad). The reactions were performed in technical triplicates and at least two biological replicates. For entire embryos, every biological replicate consists of a pool of 5-10 larvae per genotype; for heart-extracted samples, every replicate consists of cDNA derived by at least 9 hearts. Gene expression values were normalized to the zebrafish rpl13a gene.

\subsubsection{Transcriptomics}

Total RNA and library integrity were verified with LabChip Gx Touch 24 (Perkin Elmer). 100 ng of total RNA was used as input for VAHTS Stranded mRNA-seq Library (Vazyme) preparation. Sequencing was performed on the NextSeq500 instrument (Illumina) using v2 chemistry, resulting in an average of $37 \mathrm{M}$ reads per library with $1 \times 75 \mathrm{bp}$ single end setup. Quality, adapter content and duplication rates of the raw reads were determined with FastQC. Reads with a quality drop below a mean of Q20 in a window of 10 nucleotides (M. P. Davis et al., 2013) were trimmed with Reaper version 13-100. Only reads between 30 and 150 nucleotides were used in subsequent analyses and aligned with the Ensembl Zebrafish genome version DanRer11 (GRCz11.92) using STAR 2.4.0a with the parameter "outFilterMismatchNoverLmax 0.1" (Dobin et al., 2013). The number of reads aligning to genes was counted with featureCounts 1.4.5-p1 tool from the Subread package (Liao et al., 2014). Only reads mapping at least partially inside exons were kept and aggregated per gene, while reads overlapping multiple genes or aligning to multiple regions were excluded from further analyses. Differentially expressed genes were identified using DESeq2 version 1.18.1(Love et al., 2014). To remove a batch effect from the comparison, the replicates were presented to DESeq2 as covariates (DMSO_1/Inhib_1 = 1, DMSO_2/Inhib_2 = 2). Genes were classified to be differentially expressed genes (DEG) with Benjamini-Hochberg corrected P-Value $<0.05$ and $-0.59 \leq \log 2 \mathrm{FC} \geq+0.59$. The Ensembl annotation was enriched with UniProt data (release 06.06.2014) based on Ensembl gene identifiers (Activities at the Universal Protein Resource (UniProt)). The raw count matrix was batch corrected using CountClust (Dey et al., 2017) and then normalized with DESeq2. MA plots were produced to show DEG regulation per contrast.

Gene ontology enrichment of differentially expressed genes $(\log 2 \mathrm{FC}>|1|$; P adjusted $<0.01)$ was calculated using cluster profiler (v 3.15.0) (Yu et al., 2012) and with annotations derived from org.Dr.eg.db (v 3.10.0).Main overrepresented biological processes were identified and agglomerated based on manual inspection of significant Gene Ontology database gene sets with P-Value $<0.01$. 


\subsubsection{Fluorescent-activated cell sorting (FACS)}

For Fluorescence Activated Cell Sorting (FACS), hearts were manually extracted as described in section 3.2.4 and dissociated into single cell suspensions. To do so, the samples were centrifuged for 60 seconds at $5000 \mathrm{rpm}$ and the medium removed. The hearts were washed with $1 \mathrm{ml}$ HBSS (Hanks' Balanced Salt Solution). They were then incubated in $100 \mu \mathrm{l}$ Enzyme $1+5 \mu$ l Enzyme 2 (Pierce Cardiomyocytes Dissociation Kit) for 20 minutes at $30^{\circ} \mathrm{C}$ on a shaker (300-350 rpm) pipetting every 5-10 minutes to mechanically dissociate the tissues. The digestion reaction was stopped by adding $1 \mathrm{ml}$ DMEM+FBS to the tube and centrifuging for 3 minutes. Then, after discarding the supernatant, $400 \mu \mathrm{l}$ DMEM + FBS was added and the solution containing dissociated cells was passed through and collected into $40 \mu \mathrm{l}$-filtered FACS tubes.

All the samples were stained with DAPI to distinguish between live/dead cells. Together with the experimental sample, negative controls of non-fluorescent hearts or single-color fluorescent hearts were prepared in order to adjust the gate.

A gate to distinguish cells from debris on a scatter plot of forward scatter (FSC-A) amplitude vs side scatter amplitude (SSC-A) was selected. An additional gate to distinguish single cells from doublets and multimers was set. According to the experiment, $\operatorname{Tg} B A C(t c f 21: n l s-$

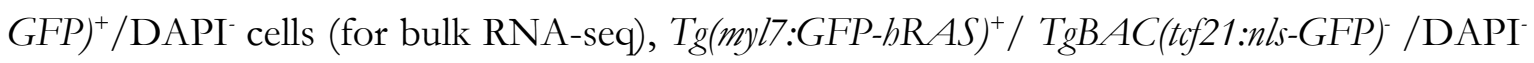
cells, were collected by FACS sorting on a BD FACSAria ${ }^{\mathrm{TM}}$ III machine. The cells were sorted directly in TRIzol for bulk-RNAseq or RT-qPCR experiments and frozen at $-80^{\circ} \mathrm{C}$.

\subsubsection{In-Fusion cloning}

Snapgene software (from Insightful Science; available at snapgene.com) was used to analyze DNA sequences and restriction sites on vectors and inserts, build final plasmid maps, and for designing amplification primers. Snapgene software to determine correct restriction enzymes, DNA digestion conditions and buffer. The restriction digestion reaction (50 $\mu$ l in total) of the plasmid (vector) was set up as follows:

\begin{tabular}{|l|l|}
\hline Component & Volume \\
\hline Plasmid DNA & $2-5 \mu \mathrm{g}$ \\
\hline 10X CutSmart buffer & $5 \mu \mathrm{l}(1 \mathrm{X})$ \\
\hline Restriction enzyme & $0.5 \mu \mathrm{l}$ each (or 5 units) \\
\hline Nuclease-free water & to $50 \mu \mathrm{l}$ \\
\hline
\end{tabular}

The amplification of desired genetic sequences (insert) was performed using PrimeStar Max polymerase (Takara Clontech). The following conditions were used for the PCR (polymerase chain reaction): 


\begin{tabular}{|c|c|c|}
\hline Temperature & Time & \\
\hline $95^{\circ} \mathrm{C}$ & 2 minutes & \\
\hline $95^{\circ} \mathrm{C}$ & 15 seconds & \\
\hline $60^{\circ} \mathrm{C}$ & 30 seconds & 35 cycles \\
\hline $72^{\circ} \mathrm{C}$ & 30 seconds $/ \mathrm{kb}$ & \\
\hline $72^{\circ} \mathrm{C}$ & 5 minutes & \\
\hline $4^{\circ} \mathrm{C}$ & Indefinite & \\
\hline
\end{tabular}

The correct digestion of the vector and the amplification of the insert were confirmed using gel electrophoresis. Briefly, the PCR product was mixed with gel loading dye and loaded on to a $1 \%$ agarose gel in 1X TAE (, containing SYBR-Safe (Life Technologies). The gel was run for $30 \mathrm{~min}$ at 150 volts. Both the digested vector and the amplified insert were cut out from the gel using a trans-illuminator. The DNA was isolated using a Gel Purification Kit (Thermo Fisher) and its concentration measured using a NanoDrop. Purified DNA products were used to set up a recombination-based cloning reaction using the enzyme In-Fusion (Takara Bio) as per the following reaction conditions:

\begin{tabular}{|l|l|}
\hline Component & Amount/volume \\
\hline Linear vector & $50-100 \mathrm{~g}$ \\
\hline PCR-amplified insert & $\begin{array}{l}\text { Depending on the size } \\
\text { (molar ratio insert:vector=3:1) }\end{array}$ \\
\hline In-Fusion Master mix & $0.4 \mu \mathrm{l}$ \\
\hline Nuclease-free water & to $2.5 \mu \mathrm{l}$ \\
\hline
\end{tabular}

The reaction was incubated for $15-20$ minutes at $55^{\circ} \mathrm{C}$ and then 10 minutes on ice. Subsequently, the reaction was transformed into E.coli cells as described above. The next morning bacterial clones were selected for a successful integration with the help of colony PCR using oligonucleotides spanning the vector-insert boundary, which amplify a DNA product only in positive clones.

\begin{tabular}{|c|c|c|}
\hline Temperature & Time & \\
\hline $95^{\circ} \mathrm{C}$ & 5 minutes & \\
\hline $95^{\circ} \mathrm{C}$ & 15 seconds & \\
\hline $58^{\circ} \mathrm{C}$ & 30 seconds & 35 cycles \\
\hline $72^{\circ} \mathrm{C}$ & 30 seconds $/ \mathrm{kb}$ & \\
\hline $72^{\circ} \mathrm{C}$ & 5 minutes & \\
\hline $4^{\circ} \mathrm{C}$ & Indefinite & \\
\hline
\end{tabular}

The plasmid DNA from positive clones was subsequently purified as described above. 


\subsubsection{TA cloning}

TA cloning was performed using the pGEM-T-easy vector kit (Promega), for different aims (sequencing of CRISPR/Cas9-induced mutations or creating templates for in vitro RNA transcription). The reaction was assembled as follows:

\begin{tabular}{|l|l|} 
Component & Volume \\
\hline 2X Ligation buffer & $2.5 \mu \mathrm{l}$ \\
\hline pGEM-T-easy vector & $0.5 \mu \mathrm{l}$ \\
\hline PCR product (insert) & $0.5-1 \mu \mathrm{l}$ \\
\hline T4 DNA ligase & $0.5 \mu \mathrm{l}$ \\
\hline Nuclease-free water & $1 \mu \mathrm{l}$ \\
\hline
\end{tabular}

The mix was incubated overnight at $4^{\circ} \mathrm{C}$ and subsequently transformed into competent cells. Competent cells were plated on agar plates covered with $\beta$-galactosidase solution. The next morning clones were selected based on their color (white clones, successful integration; blue clones, failed integration) following reaction with $\beta$-galactosidase.

\subsubsection{E. coli competent cell transformation}

Plasmids derived by different reactions (In-Fusion cloning and TA cloning, or obtained from other laboratories) were added to $20 \mu \mathrm{L}$ DH5B competent cells and incubated on ice for additional 10 minutes. Bacterial cells were placed at $42^{\circ} \mathrm{C}$ for 45 seconds (heat-shock) and transferred on ice for 2 minutes. $100 \mu \mathrm{l}$ was subsequently added to the cells and the entire mix was spread on culture plates containing Ampicillin and incubated $\sim 16$ hours at $37^{\circ} \mathrm{C}$. Positive clones were placed in $5 \mathrm{ml} \mathrm{LB}$ medium with the selection antibiotic and kept in the bacterial shaker at $37^{\circ} \mathrm{C}$ overnight. The next morning, the culture was centrifuged at $14000 \mathrm{rpm}$ for 2 min and the supernatant was discarded. The DNA contained in the pellet was isolated using GeneJET Plasmid Miniprep Kit (ThermoFisher) following the manufacturer's instructions. The plasmid DNA was then eluted in $30 \mu \mathrm{l}$ of water. The plasmid DNA was subsequently sent for sequencing to confirm the correct integration using the Sanger Sequencing methodology at Seqlab (Göttingen) or GATC Biotech (Constance).

\subsubsection{Dextran injections}

Larvae were placed laterally in low-melting agarose containing 0,01\% Tricaine (Sigma) at $96 \mathrm{hpf}$, in order to have access to the trunk. The larvae were injected with $2 \mathrm{nl}$ of fluorescein isothiocyanate (FITC)-dextran $2000 \mathrm{kDa}$ into the posterior cardinal vein on top of the yolk extension. After verifying the correct injection on a fluorescence stereomicroscope, larvae where embedded for confocal imaging in $1 \%$ low melting agarose and $0.2 \%$ tricaine to stop the heart. 


\subsubsection{Immunostaining}

Whole mount immunostaining was performed as described previously (Gunawan et al., 2019), with some modifications. Embryos or larvae were euthanized with $0.2 \%$ Tricaine and then fixed in 4\% PFA for 2 hours at room temperature. Embryos were then washed in PBS several times and the yolk was removed using forceps to facilitate the penetration of the antibody. Embryos/larvae were permeabilized with $10 \mu \mathrm{g} / \mathrm{ml}$ Proteinase $\mathrm{K}$ for a time dependent on the developmental stage, and then washed several times in PBDT. The blocking step was performed in PBDT and 5\% goat serum. Primary and secondary antibody incubations were performed overnight at $4^{\circ} \mathrm{C}$. Between the two incubations, the samples were washed extensively (minimum 4x20 minutes) in PBDT. Embryos or larvae were incubated with 1 $\mu \mathrm{g} / \mathrm{ml} \mathrm{DAPI}$ together with secondary antibody. On the following day, they were washed $4 \times$ 15 min in PBS $+0.1 \%$ TritonX and mounted in agarose and genotyped after imaging.

Primary antibodies dilutions used are as follows: GFP (AvesLab, 1:400), Elastin2 (1:100), tRFP (Evrogen, 1:200), anti-dsRed (Takara Bio Clontech, 1:200), anti-Alcama/Dm-Grasp (DSHB, 1:50), anti-p-Smad3 (Abcam, 1:100) and anti-pSmad1/5/8 (Cell Signaling Technology, 1:100). Secondary antibodies (Secondaries Alexa FluorTM 488-568-647 IgG (H+L), Thermo Fisher Scientific) were used at 1:500 concentration. Elastin2 antibody was purified from the serum stock described previously (Miao et al., 2007).

\subsubsection{EdU incorporation assay}

Embryos were incubated in $0.5 \mathrm{mM}$ EdU (Thermo Fisher) $/ 0.5 \%$ DMSO dissolved in PTU water from 24 to $36 \mathrm{hpf}$ or 48 to $72 \mathrm{hpf}$, according to the experiment. Embryo or larvae were then euthanized, followed by fixation and whole-mount immunostaining (as described above). Following the 4 washes post-second antibody incubation, the Click-iT reaction was performed, following the Click-iT EdU Cell Proliferation Kit for Imaging, Alexa Fluor 647 dye manufacturer's instructions. As I observed different efficiencies of cell labeling with different batches of EdU, all the samples of all the replicates for each experiment were treated with the same batch of EdU.

\subsubsection{TUNEL assay}

Embryos were fixed at $80 \mathrm{hpf}$ with 4\% PFA for 2 hours at room temperature. After several washes in $0.1 \%$ PBST, the larvae were gradually dehydrated with progressive concentrations of methanol in $0.1 \%$ PBST and incubated in $100 \%$ methanol at $-20^{\circ} \mathrm{C}$ for at least 24 hours. Larvae were then treated with $10 \mathrm{~g} / \mathrm{ml}$ Proteinase $\mathrm{K}$ and the antibody staining was performed as described previously. After the secondary antibody incubation, TUNEL (Terminal deoxynucleotidyl transferase dUTP nick end labeling) assay was performed. First, samples were incubated in $0.1 \%$ sodium citrate in PBST for 2 minutes on ice for permeabilization. Following 
3 washes in $0.1 \% \mathrm{PBS}+0.1 \%$ TritonX, some samples were placed in a different tube for the positive control treatment with DNase I (1hour, $37^{\circ} \mathrm{C}$ ) and washed thoroughly. The samples were then incubated with TUNEL-label solution for 1 hour at $37^{\circ} \mathrm{C}$, according to manufacturer's instructions. Samples were washed in PBS $+0.1 \%$ TritonX several times before imaging.

\subsubsection{Whole mount in situ hybridization}

DNA template for in situ RNA probes derived from PCR products obtained by wild-type cDNA, using a reverse oligonucleotide containing the $\mathrm{T} 7$ promoter sequence necessary for $\mathrm{T} 7$ polymerase activity. With these templates, Digoxigenin (DIG)-labeled probes were transcribed in vitro, using T7 polymerase (Promega) and DIG RNA labeling kit (Roche). Larvae were then euthanized at the desired stage and fixed with $4 \%$ PFA overnight at $4{ }^{\circ} \mathrm{C}$. In situ hybridization was performed as described previously (Thisse and Thisse, 2008). Anti-GFP primary antibody (1:300, AvesLab) labeling the endothelium of $\mathrm{Tg}(\mathrm{k}$ drl:EGFP) larvae, or MF-20 primary antibody (1:100, DSHB) to label the myocardium, were added together with the anti-Dig antibody. The secondary antibodies were added after BM Purple treatment, for 2 hours at room temperature. For fluorescent in situ hybridization (FISH), the same protocol of the whole mount bright-field in situ was conducted with some changes. During the first day, embryos were incubated in 1\% $\mathrm{H}_{2} \mathrm{O}_{2}$ for 30 min. The next day, embryos were incubated with the primary antibodies anti-DIGPOD (Roche, 1:500) and anti-dsRed (Takara Bio Clontech, 1:200). The next day, after six washes in PBST, embryos were incubated with Amplification solution (TSA ${ }$ ) fluorescein detection kit) for 2 min. FITC (1:100) was added to the Amplification solution for developing the signal. The embryos were then incubated in the mix for $30 \mathrm{~min}$ in the dark at RT and washed in PBST. Embryos were then incubated 2 hours with secondary antibody solution (goat anti-rabbit 568, 1:500) and $1 \mu \mathrm{g} / \mathrm{ml} \mathrm{DAPI}$, and then extensively washed in PBST before imaging.

Embryos were imaged on a Nikon SMZ25 stereomicroscope with a $2 \times / 0.3$ objective or LSM700 Axio Imager 40x objective and, if necessary, genotyped afterward.

\subsubsection{In vitro RNA transcription}

To obtain RNA to inject at one cell stage, DNA template for Cas 9 mRNA and $f b \ln 5 \mathrm{mRNA}$ was obtained from linearized plasmids. $f b \ln 5$ coding sequence was amplified by wild-type cDNA with the following primers fbln 5 ATG fw 5'-ATGT'TTGTTGAACTACGTGGC3',fbln5 STOP rv 5'-TCAGAATGGATGTTCGGAGAC-3' and cloned into the pCS2 ${ }^{+}$plasmid with In-Fusion cloning (described in 3.2.8). To synthesize Cas9 mRNA, pT3TS-nCas9n (Addgene) was used as a template. The mRNA were synthesized in vitro using the mMESSAGE 
mMACHINE TM T7 (for fbln5) or T3 (for Cas9) transcription Kits and purified with RNA Clean \& Concentrator.

\subsubsection{CRISPR/Cas9-mediated mutagenesis}

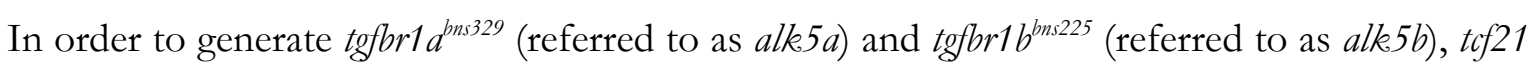
bnst27, wt1 $a^{\text {bns } 428}$ mutant lines, a CRISPR design tool (https://chopchop.cbu.uib.no/) was used to design sgRNAs. sgRNAs were assembled as described (Gagnon et al., 2014; Varshney et al., 2015) with the following sequences: $\operatorname{tg} b r 1 a^{\text {bns } 329}$, a sgRNA targeting exon 1 (targeting sequence: GGATGATCTTTACCCCC); tgfbr1 $b^{\text {bns } 225}$, a sgRNA targeting exon 4 (targeting sequence: GAGGAGCGCTCCTGGTTC); $\operatorname{tcf} 2^{\text {bns } 427}$, a sgRNA targeting exon 1 (targeting sequence: CGCAGCTAACGCGCGCGAGA), wt1 a bns 28 , 3 sgRNAs were injected to delete the promoter region (targeting sequences: CAACTGGACAGCTTGGCCTA, AAAGGCGTCTAATAGAC AAC, GGCAGTGCCACTCTTGCCAG). 50 pg sgRNA and 300 pg Cas 9 mRNA were injected into 1-cell stage embryos. The mutants were genotyped using high resolution melt analysis (HRMA) (Eco-Illumina) with the primers described in section 3.1.13.

The tofbr1 $a^{\text {bns } 329}$ fish carry a 2 bp insertion and a 6 bp deletion in exon 1, while the $\operatorname{tg} f b r 1 b^{\text {bns } 225}$ fish carry a $3 \mathrm{bp}$ insertion and an $11 \mathrm{bp}$ deletion in exon $4 . \operatorname{tcf} 21^{\mathrm{bns} 427}$ animals carry a $10 \mathrm{bp}$ deletion in exon 1 . $w t 1 a^{\text {bns } 428}$ fish carry a $\sim 8 \mathrm{~kb}$ deletion in the promoter region, including the ATG.

\subsubsection{Generation of transgenic lines}

To generate $\operatorname{TgB} A C\left(\operatorname{tg} b r 1 b: E G F P\right.$, cryaa:CFP) ${ }^{\text {bns } 330}$, abbreviated $\operatorname{TgB} A C(a l k 5 b$ :EGFP), I used the BAC clone CH1073-59L16 (BACPAC Resources Center), containing $37.3 \mathrm{~kb}$ of the alk.5b locus. All the steps of recombination were performed as previously described (Bussmann and Schulte-Merker, 2011), with the addition of a cryaa:CFP-iTol2 cassette to screen transgenic animals. The following homology arms were used to amplify the EGFP_Kan cassette and recombine it in place of the original alk.5b ATG: alk.5b HA1 GFP fw 5'-

ACTGGAGTCCAGCAGGAGAACAGAAGAGGAGCGGGATTATCTCCAGGAGGAC CATGGTGAGCAAGGGCGAGGAG-3', alk5b HA2 Kan rv 5'ACCAGTCGACACGGCACTGCCTCCATCATCATCATCTTCATCATCATCTTTTTCCA GAAGTAGTGAGGAG -3'. To generate Tg(fli1 a:tgfbr1b-P2A-mScarlet-Hsa.HRAS) $)^{\text {bns } 421}$, abbreviated $\mathrm{Tg}$ (fli1 a:alk 5 b-mScarlet), tgfbr1b coding sequence was amplified using the primers alk.5b ATG fw 5'- GGATGAAGATGATGATGAAGATG -3' and alk.5b noSTOP rev 5'CATCTTGATTCCCTCCTGCT -3' and cloned downstream of the flit $a$ enhancer-promoter sequence (fli1ep) (Villefranc et al., 2007). To generate the myl7:ERK-KTR-Clover-tag H2B- 
mScarlet plasmid, ERK KTR mClover was amplified from plasmid \#59150 (Addgene) using the following primers: GCAAAGCAGACAGTGAACAAGCTTGCTAGCCCACCATG AAGGGCCGAAAGCCTCGGG and GT'TAGTAGCTCCGCTTCCGTCGACGGCGG CGGTCACGAACTCCAGCAGG and cloned downstream of the myl7 promoter into a Tol2 vector. The Tol2 vector contained P2A and H2B tag sequences.

To generate $T g(f l i 1 a: f b \ln 5$,EGFP), abbreviated $T g(f l i 1 a: f b \ln 5)$, fbln 5 coding sequence was amplified with the primers fbln 5 ATG fw 5'-ATGTTTGTTGAACTACGTGGC-3', fbln 5 STOP rv 5'-TCAGAATGGATGTTCGGAGAC-3'. The fbln5 CDS was cloned in the fli1 aep:EGFP, $m$ Cherry (Nicoli et al., 2010) plasmid, in place of the mCherry. All cloning experiments, except for the BAC, were performed using In-Fusion Cloning.

\subsubsection{Genotyping High-Resolution Melt (HRM) analysis}

To genotype embryos/larvae or adult fins, genomic DNA was isolated placing the sample in a tube containing $50 \mathrm{mM} \mathrm{NaOH}$. The tubes were incubated at $95^{\circ} \mathrm{C}$ for 10 minutes and $10 \%$ volume of $1 \mathrm{M}$ Tris- $\mathrm{HCl}(\mathrm{pH}=8)$ to stop the digestion reaction. The samples were stored at $4^{\circ}$ C. The genomic DNA was then screened for mutations with HRM analysis. During an HRM reaction, a region of interest is amplified and its melting curve generated. HRM mix was prepared as follows for each sample:

\begin{tabular}{|l|l|}
\hline Component & Volume \\
\hline $\begin{array}{l}\text { DyNAmo ColorFlash } \\
\text { SYBR Green qPCR Mix }\end{array}$ & $4.5 \mu \mathrm{l}$ \\
\hline $\begin{array}{l}\text { Mix of forward and } \\
\text { reverse primers }(\mathbf{0 . 7} \boldsymbol{\mu M} \text { each) }\end{array}$ & $4.5 \mu \mathrm{l}$ \\
\hline genomic DNA & $1 \mu \mathrm{l}$ \\
\hline
\end{tabular}

The reaction conditions were as follows:

\begin{tabular}{|c|c|c|}
\hline Temperature & Time & \\
\hline $95^{\circ} \mathrm{C}$ & 7 minutes & \\
\hline $95^{\circ} \mathrm{C}$ & 10 seconds & Amplification \\
\hline $60^{\circ} \mathrm{C}$ & 15 seconds & 35 cycles \\
\hline $95^{\circ} \mathrm{C}$ & 15 seconds & \multirow{3}{*}{$\begin{array}{l}\text { Melting } \\
\text { curve }\end{array}$} \\
\hline $60^{\circ} \mathrm{C}$ & 10 seconds & \\
\hline $95^{\circ} \mathrm{C}$ & 15 seconds & \\
\hline
\end{tabular}

\subsubsection{Transmission electron microscopy (TEM)}

Entire larvae were collected at $72 \mathrm{hpf}$ and the final portion of the trunk was cut and used for genotyping; adult hearts were manually dissected and the surrounding tissues removed. The larvae were fixed in cold 1\% PFA, 2\% glutaraldehyde in $0.1 \mathrm{M}$ sodium cacodylate buffer for 30 
minutes on ice, while adult hearts in $4 \%$ paraformaldehyde with $2.5 \%$ glutaraldehyde in $0.05 \mathrm{M}$ HEPES buffer for 2 hours at room temperature, and both were subsequently stored at $4{ }^{\circ} \mathrm{C}$. Samples were washed in $0.1 \mathrm{M}$ sodium cacodylate buffer (larvae) or 0.05 M HEPES buffer (hearts) and postfixed in $2 \%(\mathrm{w} / \mathrm{v}) \mathrm{OsO}_{4}$. After washing with deionized water, blocks were stained with $2 \%$ uranyl acetate. Samples were dehydrated with a graded series of washes in acetone, transferred to acetone/Epon solutions, and eventually embedded in Epon. Tissue semi-thin sections (900 nm thick) were obtained in a Ultracut E microtome (Reichert-Jung, Leica) and stained with Richardson staining solution (Richardson et al., 1960). Ultra-thin sections $70 \mathrm{~nm}$ thick were then collected on 2x1 copper slot grids. Sections were examined with a Jeol JEM-1400 Plus transmission electron microscope (Jeol, Japan), at an accelerating voltage of $120 \mathrm{kV}$. Digital images were recorded with an EM-14800Ruby Digital CCD camera unit (3296px x 2472px).

\subsubsection{Chemical treatments}

For all the chemical treatments, embryos or larvae were treated with the different compounds dissolved in egg-water at different concentrations, to assess toxicity and effectiveness. The final concentration chosen was the lowest effective dose, and it was not resulting in off-target developmental defects. Control embryos were treated for the same time at the same concentration of DMSO.

The Alk5 inhibitor (E-616452, Cayman Chemical), the FGFR inhibitor (SU-5402, Sigma), and the VEGFR inhibitor (SU-5416, Sigma) were dissolved in DMSO at a concentration of $10 \mathrm{mM}$ and frozen in single aliquots. Embryos were treated a final concentration of $2.5 \mu \mathrm{M}(\mathrm{E}-616452)$ or $1 \mu \mathrm{M}$ (SU-5402 and SU-5416). For Smad3 inhibitor experiments, SIS3 (Jinnin et al., 2006; Dogra et al., 2017) was dissolved in DMSO at a concentration of 5mM. Embryos were treated with $1.5 \mu \mathrm{M}$ of the inhibitor or DMSO from $36 \mathrm{hpf}$ until $75 \mathrm{hpf}$, protected from light. For Lyso'Tracker and DAF-FM experiments, $95 \mathrm{hpf}$ live larvae were incubated at $28^{\circ} \mathrm{C}$ for 1 or 4 hours, respectively, in egg water containing $10 \mu \mathrm{M}$ LysoTracker Deep Red (Thermo Fisher Scientific) or $5 \mu \mathrm{M}$ DAF-FM DA (Sigma Aldrich). Larvae were rinsed several times with fresh water before imaging.

For cell ablation experiments, Metronidazole (Mtz) solution was freshly dissolved in DMSO at $1 \mathrm{M}$ concentration and subsequently diluted in egg-water to $5 \mathrm{mM}$. Embryos and larvae were treated in different time windows (50-80 hpf, 50-96 hpf, 96-144 hpf) according to the experiments. The larvae were then immediately imaged, or rinsed several times with fresh water before placing them back to the incubator for "wash-out" experiments. 


\subsubsection{Confocal microscopy}

For live confocal imaging, embryos or larvae were embedded in 1\% low-melting agarose/egg water with $0.2 \%$ tricaine to stop the heart. To image immunostained animals, samples were mounted in 1\% low-melting agarose. All embryos or larvae were imaged with LSM700 Axio Imager, LSM800 Axio Examiner or LSM880 Axio Examiner confocal microscopes with a W Plan-Apochromat $40 \times / 1.0$ or W Plan-Apochromat 20×/1.0 dipping lenses. All images were acquired using the Zeiss ZEN program.

Unless otherwise stated, embryos from $54 \mathrm{hpf}$ onwards were imaged in ventral view to expose the heart. Embryos up to $36 \mathrm{hpf}$ were euthanized and fixed with $4 \% \mathrm{PFA}$ overnight at $4^{\circ} \mathrm{C}$; when needed they were immunostained according to the experiment. The yolk of the embryos was then manually removed. Before mounting the embryos, the trunks were removed. The images of the embryos were taken accessing to the heart from the region where the yolk was previously located.

Time-lapse videos were acquired mounting the embryos in $0.8 \%$ low-melting agarose/PTU water with $0.01 \%$ tricaine in 4-well Chambered Coverglass (Thermo Fisher Scientific) and covered with PTU water containing either $0.1 \%$ DMSO or $2.5 \mu \mathrm{M}$ Alk5 inhibitor. Stacks of the OFT and ventral artery were acquired every 45 minutes for 24 cycles from 56 hpf. Only fish that survived until the end of the time-lapse were processed for further analyses.

\subsubsection{Photoconversion}

Tg(fli1a: Kaede) (Hatta et al., 2006) embryos were treated with Alk5 inhibitor or DMSO as described above and, at $54 \mathrm{hpf}$, embedded in 1\% low-melting agarose $+0.2 \%$ tricaine to stop the heart for the duration of the imaging. Photoconversion was done on a LSM880 Axio Examiner confocal microscope with a W Plan-Apochromat 20×/1.0 immersion lens. Embryos were imaged with 488 and $561 \mathrm{~nm}$ lasers to select the region of interest (ROI) and confirm the absence of red signal before photoconversion. The ROI was exposed to 3 scans ( 4.5 seconds) of the $405 \mathrm{~nm}$ laser to perform photoconversion. Another scan with only 488 and $561 \mathrm{~nm}$ lasers was eventually performed to verify the efficiency of the photoconversion. Embryos were removed from the agarose, and -after heartbeat recovery- incubated at $28^{\circ} \mathrm{C}$ in separate wells of a 24-well plate. According to the experiment, embryos were kept in the incubator until 56 or 74 hpf.

\subsubsection{Heart function measurements}

To realize videos of beating hearts, fish were mounted in $0.8 \%$ low-melting agarose/ egg water, containing $0.01 \%$ tricaine or without tricaine for heart rate measurements. Videos were acquired with an exposure time of $10 \mathrm{~ms}$, at 100 frames/s for 150 frames, covering approximately 3-4 heartbeats; for heart rate measurements, videos were acquired with $5 \mathrm{~ms}$ 
exposure for 5000 frames (approximately $30 \mathrm{sec}$ ). Light intensity was kept to a minimum to avoid light-induced twitching. Videos were acquired with an LD C-Apochromat 40×/1.1 water immersion lens (Zeiss Cell Observer spinning disk microscope with a CSU-X1 scanner unit [Yokogawa] and Hamamatsu ORCAflash4.0 sCMOS cameras).

For OFT expansion measurements, the areas of the OFT during max and min expansion were calculated using ImageJ software as an average of four heart beats. For fractional shortening measurements, the width of the ventricle was calculated during diastole and systole and averaged across four heart beats. OFT expansion and ventricular fractional shortening were calculated using the formulas below:

OFT expansion $\%=\frac{\text { area } \max \text { expansion }- \text { area } \min \text { expansion }}{\text { area } \min \text { expansion }} \times 100$

Fractional shortening $\%=\frac{\text { width at diastole }- \text { width at systole }}{\text { width at diastole }} \times 100$

For the heartbeat, the beats per minute (bpm) were calculated using the following formula:

$$
\text { bpm }=\frac{\# \text { heart contraction }}{\text { time }(s)} \times 60(s)
$$

\subsubsection{Imaging processing and analysis}

CM, epicardial, EC and SMC cell counting was performed with Imaris (Bitplane) using endogenous nuclear markers (i.e., $\mathrm{Tg}(m y l 7: n l s-d s r e d) ; \operatorname{Tg}(m y l 7: H 2 B-G F P) ; \operatorname{TgB} A C(\operatorname{tc} 21$ :nls-GFP)) or combining the cytoplasmic marker with DAPI staining (i.e., Tg(kdrl:EGFP) and $\operatorname{TgBAC}($ pdgfrb:EGFP); $\operatorname{Tg}(k d r l: \operatorname{TagBFP}))$.

The quantification of the displacement length of photoconverted cells was performed with Imaris (Bitplane) in 3D. The distance of the photoconverted Red $^{+}$cell from the basis of the OFT at 54 hpf was measured and then subtracted from the distance of the Red $^{+}$cell farthest from the OFT valve at $74 \mathrm{hpf}$.

For the quantification of Eln $2^{+}$cells around the OFT, Eln2 ${ }^{+} \mathrm{DAPI}^{+} \mathrm{GFP}^{-}$cells were manually selected, starting at the OFT mid-sagittal plane and proceeding 2 planes up and 2 down $(2 \mu \mathrm{m}$ increment). An average per plane was used for the analyses. The percentage of Eln $2^{+}$cells was calculated as $\left(\mathrm{E}^{-n} 2^{+} \mathrm{DAPI}^{+} \mathrm{GFP}^{-}\right.$cells $) /\left(\mathrm{DAPI}^{+} \mathrm{GFP}^{-}\right.$cells $)$x100.

To quantify the $\mathrm{p}$-Smads immunostaining intensities, mean grey values of $\mathrm{p}$-Smads were calculated with Fiji ImageJ in $>7$ cells from $>3$ single optical slices per embryo. p-Smad/DAPI ratios were generated for each cell and an average of all the cells/embryo was plotted in the graph. 


\subsubsection{Statistical analysis}

All statistical analyses were performed in GraphPad Prism (Version 6.07). Parametric $t$-tests or Mann Whitney tests were performed following assessment of the distribution of the data with D’Agostino-Pearson normality test. For multiple comparisons, One-way ANOVA, followed by Tukey's post-hoc test, or Kruskal-Wallis test, followed by Dunn's post-hoc test, were performed. Data in graphs are mean \pm SD. $P$ values from statistical tests are shown in the figures.

Certain lines in this section have been quoted verbatim from Boezio et al., eLife 2020;9:e57603 for the scientific accuracy of the terms. 


\section{$4 \quad$ Results}

\subsection{The endothelium-smooth muscle crosstalk in the developing zebrafish cardiac outflow tract is orchestrated by TGF- $\beta$ /Alk5 signaling}

Part of chapter 4.1 has been published as an article in the journal eLife (Boezio et al., eLife 2020; DOI: 10.7554/eLife.57603). My contribution to the article is described as follows: conceptualization, investigation, methodology, formal analysis, validation, visualization, writing - original draft, review, and editing.

\subsubsection{The TGF- $\beta$ pathway is active in the developing heart and enriched in the forming OFT}

In order to understand the role of TGF- $\beta$ signaling during zebrafish cardiovascular development, I focused on its main receptor type I, Alk5. Mammalian Alk.5 has two paralogs in zebrafish, alk.5a and alk.5b (Figure 12A). The two proteins Alk5a and Alk5b exhibit a high degree of identity (79.6\% identity; calculated with Clustal2.1 (Goujon et al., 2010; Sievers et al., 2011)). Notably, Alk5b shares a slightly higher identity with the mouse (80.8\%) and human (81.4\%) proteins, than with the zebrafish Alk5a. The two zebrafish proteins are more similar in the intracellular domain (amino acid 175-end), while they differ substantially in the ligandbinding domain (amino acid 1-126; Figure 12B). This difference in the extracellular domain might reflect a different specificity for ligand binding between the two receptors.

A

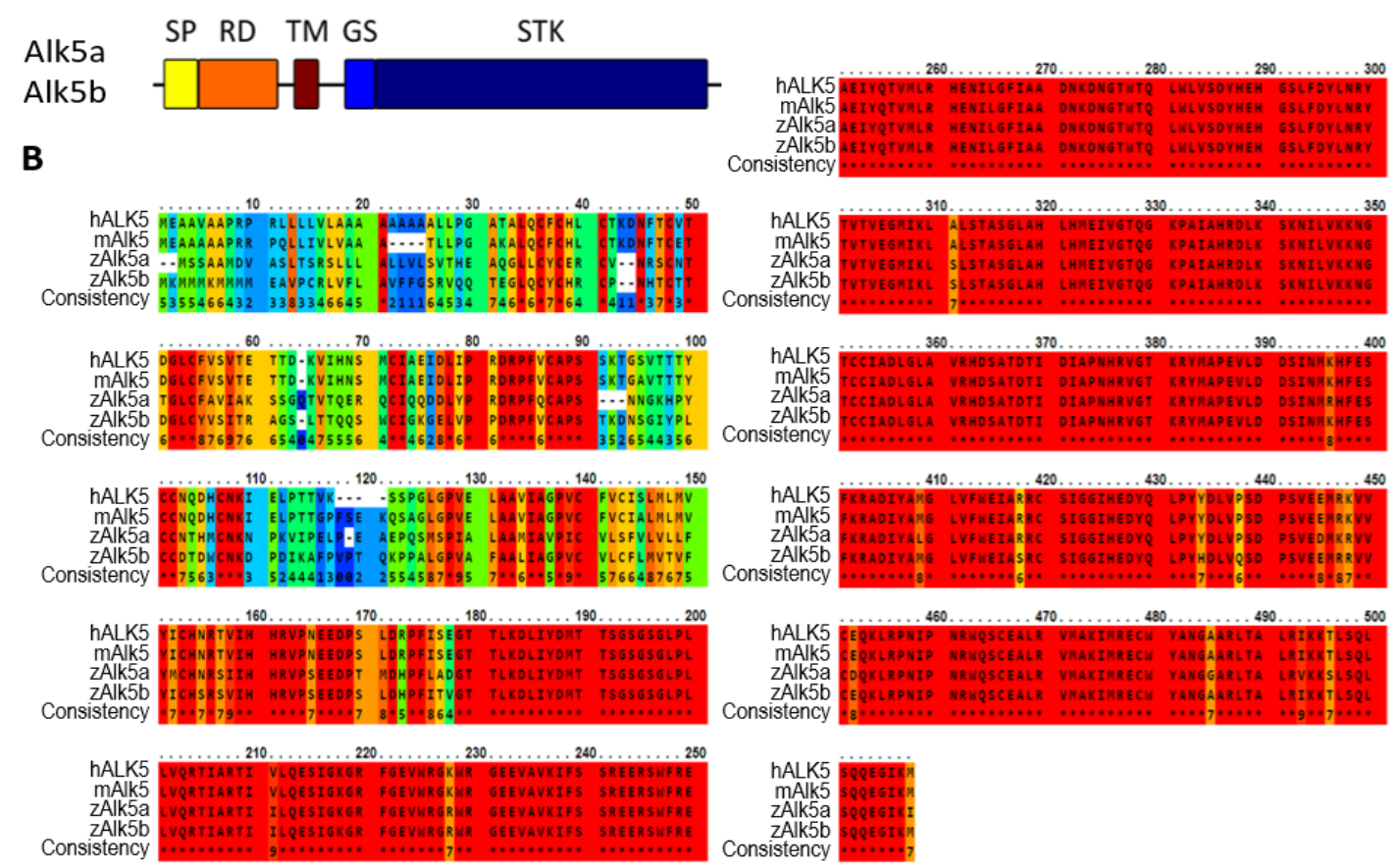

Figure 12: Representation of Alk5 protein structure and Alk5a-Alk5b sequence similarity. 
Figure 12: Representation of Alk5 protein structure and Alk5a-Alk5b sequence similarity. (Previous page) A) Schematic of Alk5 proteins depicting the functional domains: SP, signaling peptide; RD, receptor domain; TM, transmembrane domain; GS, glycine-serine rich domain; STK, serine-threonine kinase domain. B) PRALINE (Simossis and Heringa, 2005) multiple sequence alignment of human, mouse, and zebrafish Alk5 proteins. The GS (175-204) and STK (205-end) domains are the most conserved. The "Consistency" row indicates the similarity of the amino acidic residues from 0 (blue, not conserved) to 10 (*, red, conserved).

I first performed whole-mount in situ hybridization experiments at different developmental stages and I could observe the expression of both alk $5 a$ and alk $5 \mathrm{~b}$ starting at 24 hpf prominently in the neural tube and the head (Figure 13 A, B). Later during the development, alk.5b expression persists, while alk. $5 a$ decreases over time. At 54 hpf, alk. $5 b$ was also faintly expressed in the heart, enriched in the ventricle and the OFT (Figure 13 C, C'), and later in the gut region (Figure 13 D, D').
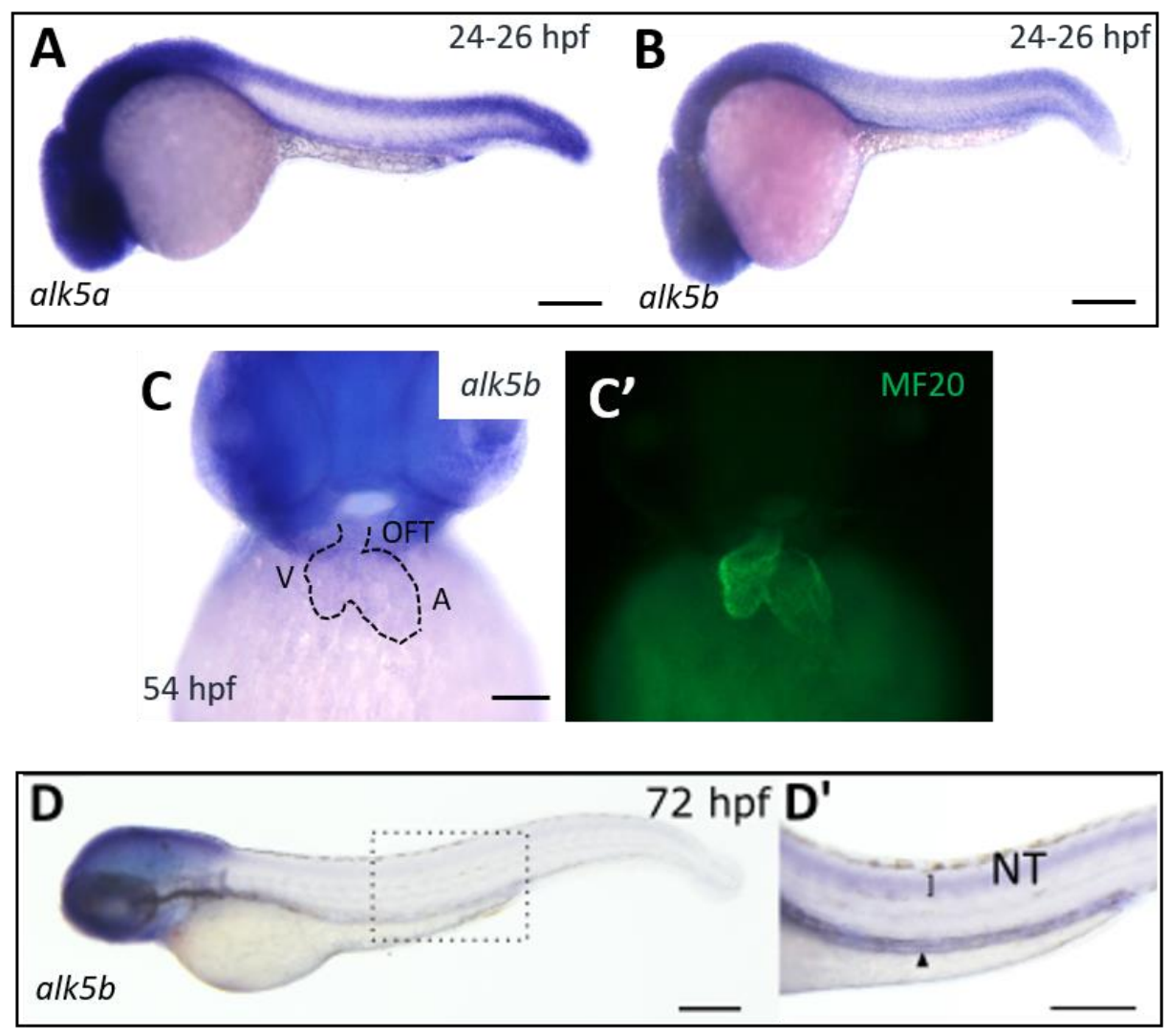

Figure 13: Expression pattern of alk5a and alk $5 b$ during zebrafish development.

A-B) Whole-mount in situ hybridization (ISH) of alk.5a and alk.5b at 24-26 hpf, showing mRNA expression in the neural tube and the head. C, C') alk.5b expression in the zebrafish $54 \mathrm{hpf}$ heart (as highlighted by MF20 immunostaining, C'), showing faint staining by in situ hybridization in the ventricle and the OFT. A, atrium; V, ventricle. D) In situ hybridization of alk $5 \mathrm{~b}$ in $72 \mathrm{hpf}$ larvae, showing staining in the neural tube (NT), the head, and the gut (arrowhead). Figure adapted and reprinted with permission from (Boezio et al., 2020). License: CC BY 2.0.

To obtain cellular resolution, I generated a transgenic reporter line for alk.5b expression, $\operatorname{TgB} A C($ alk.5b:eGFP), using the bacterial artificial chromosome (BAC) technique. The 
expression of the reporter was generally very low and often required the amplification of the signal by immunostaining. Consistent with alk.5b endogenous expression, alk.5b reporter expression appeared in the neural tube by $24 \mathrm{hpf}$ (Figure $14 \mathbf{A}, \mathbf{A}^{\prime}$ ). Focusing on the cardiovascular system, I could not detect GFP signal in any blood vessel in the trunk or the brain, but I observed alk. $5 \mathrm{~b}$ reporter expression in the heart from $2 \mathrm{dpf}$. Starting at $72 \mathrm{hpf}$, alk. $5 \mathrm{~b}$ reporter expression becomes prominent in the outflow tract (OFT), in both ECs and SMCs (Figure 14 B-B'). The expression of the reporter persists in the adult OFT (3 months postfertilization, mpf), mostly restricted to SMCs (Figure 14 C).
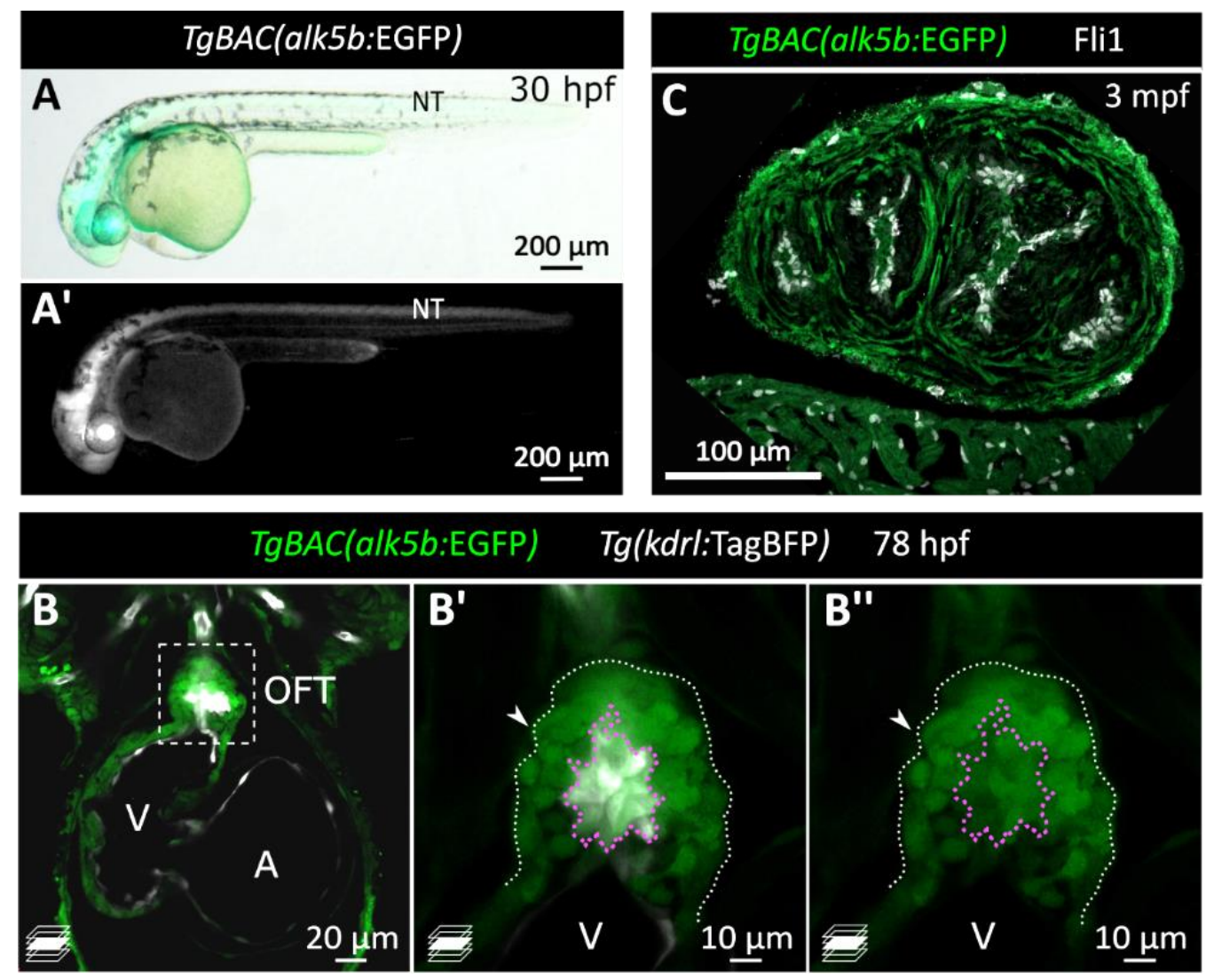

Figure 14: Expression pattern of $T g B A C$ (alk5b:EGFP) during zebrafish development.

A, A') The expression of $\operatorname{TgBAC}($ alk:5b:EGFP) is localized in the neural tube (NT) at $30 \mathrm{hpf}$ and in all the nervous system, but it is not detectable in any vascular bed of the trunk. B-B") alk $5 b$ reporter expression in the $78 \mathrm{hpf}$ zebrafish heart is enriched in the OFT and localized in SMCs (arrowhead, B', B") and ECs (white, pink dashed lines). A, atrium; V, ventricle. C) At 3 months post-fertilization (mpf) the expression of TgBAC(alk5b:EGFP) appears enriched in OFT SMCs, rather than in ECs (Fli1+ cells, white). Figure adapted and reprinted with permission from (Boezio et al., 2020). License: CC BY 2.0.

The expression of the receptors does not always correlate with the activation of the pathway, as many regulatory steps are needed to finely tune the signaling. Therefore, to investigate the pathway activity, I assessed the activation of Smad3. Smad3, together with Smad2, is the main downstream effector of TGF- $\beta$ signaling and, upon ligand binding, it becomes phosphorylated by the receptors type I and II. Once phosphorylated, it translocates to the nucleus where it 
binds to consensus sequences on several promoters, acting -together with other co-factors- as a transcription factor. I first used a Smad3-responsive element reporter zebrafish transgenic line, (Tg(12xSBE:EGFP); Casari et al., 2014), which activates GFP expression downstream of 12 repetitions of Smad3 binding sequences. Starting at $24 \mathrm{hpf}$, I observed an enriched signal in the OFT (Figure 15 A, A'), still present at 75 hpf (Figure 15 B-C').

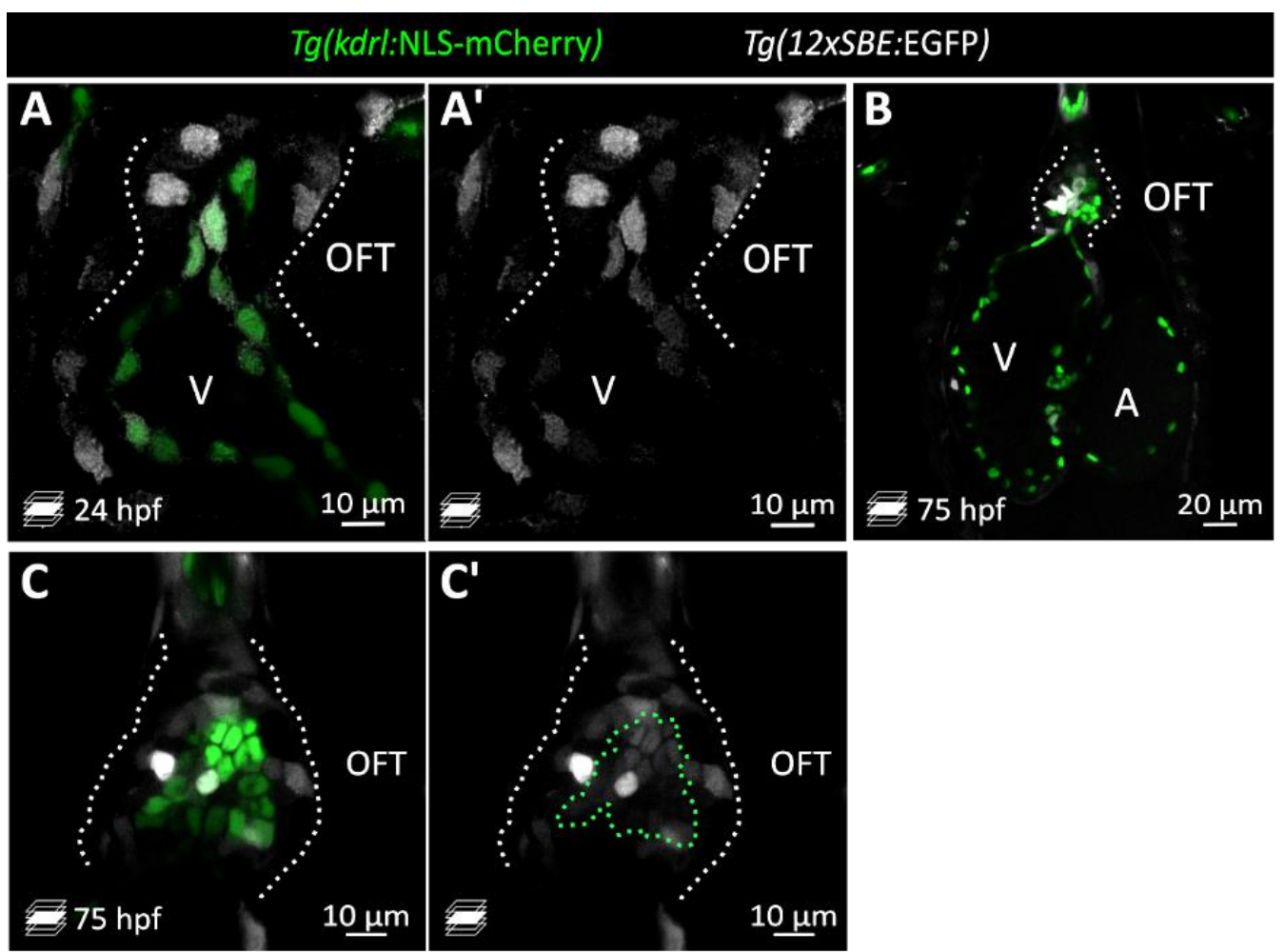

Figure 15: $\operatorname{Tg}(12 \times S B E: E G F P)$, a Smad3 reporter line, is enriched in the developing OFT

A, A') The expression of $\mathrm{Tg}(12 x \mathrm{SBE}$ :EGFP) (white) is enriched in the $24 \mathrm{hpf}$ OFT and localized in ECs (green) and surrounding cells. B-C') Smad3 reporter expression in the zebrafish $75 \mathrm{hpf}$ heart is enriched in the OFT (B) and localized in SMCs (white dashed line) and in ECs (green dashed lines) (C, C'). A, atrium; V, ventricle. Figure adapted and reprinted with permission from (Boezio et al., 2020). License: CC BY 2.0.

To assess the endogenous activation of Smad3, I performed immunostaining for the phosphorylated form of Smad3 (p-Smad3). I detected p-Smad3 immunostaining in the heart starting at $24 \mathrm{hpf}$ (Figure 16 A, A'), and until 75 hpf (latest stage analyzed, Figure 16 B, B'), consistent with the expression of the $\mathrm{Tg}(S B E$ :EGFP) reporter. The $\mathrm{p}$-Smad3 signal in endothelial cells was 1.7 fold enriched in the OFT compared with the rest of the heart (Figure 16 C). Notably, at early embryonic stages ( $24 \mathrm{hpf}$ ) the p-Smad3 signal was significantly stronger in OFT ECs than in the adjacent OFT cells (2 folds higher in ECs; Figure 16D). At 75 hpf, consistent with the Smad3 reporter expression, p-Smad3 endothelial enrichment was lost (Figure 16 A-B', D). 

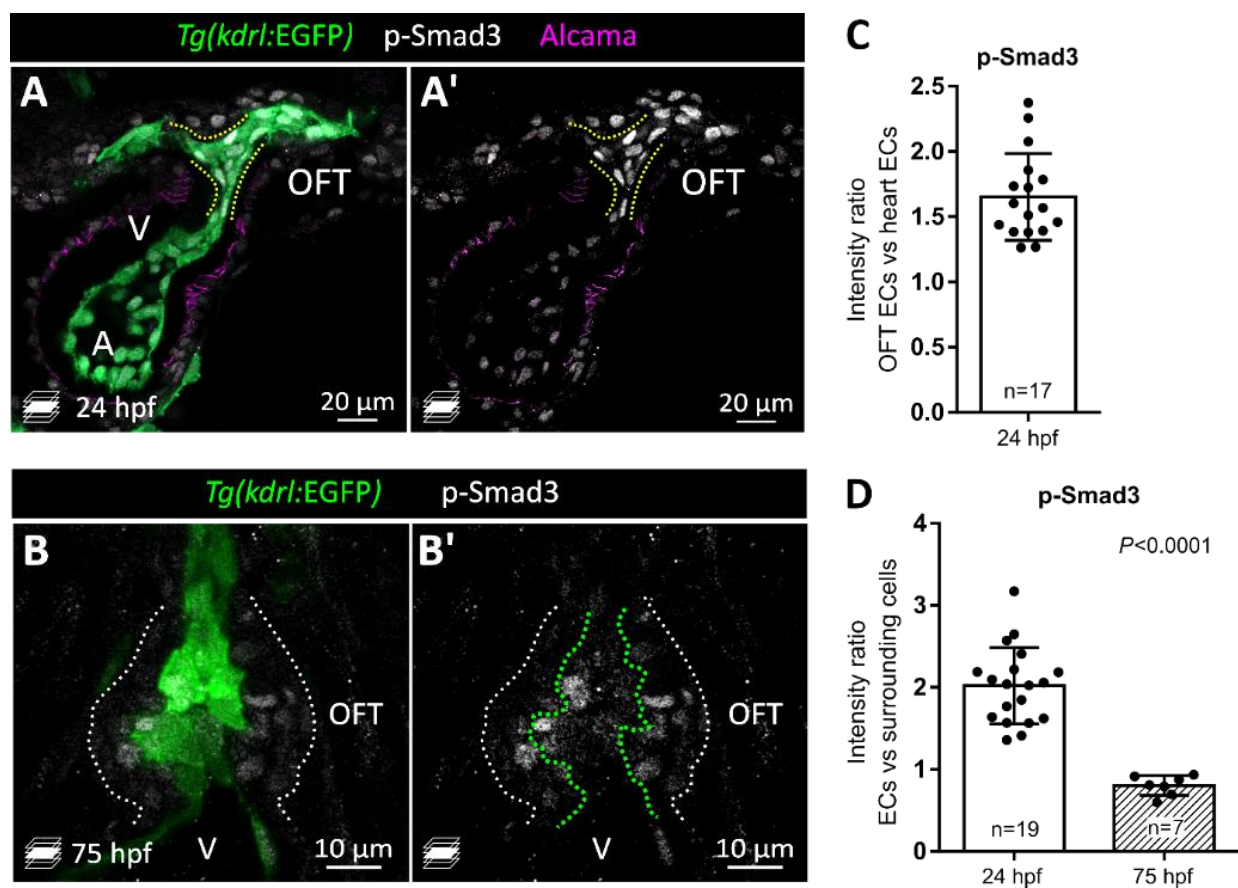

Figure 16: The Smad3 signaling is active in OFT ECs from early developmental stages

A, A') p-Smad3 immunostaining (white) is enriched in the $24 \mathrm{hpf} \mathrm{OFT} \mathrm{and} \mathrm{localized} \mathrm{in} \mathrm{ECs} \mathrm{(yellow} \mathrm{dashed}$ line) and surrounding cells. B-B') p-Smad3 immunostaining (white) is detectable in the $75 \mathrm{hpf}$ OFT and localized in SMCs (white dashed line) and in ECs (green dashed lines). A, atrium; V, ventricle. C) p-Smad3 intensity ratio between the ECs in the OFT compared with ECs in the rest of the heart shows OFT enrichment. D) p-Smad3 intensity ratio between ECs and surrounding cells at 24 and 75 hpf. Mean \pm SD; $P$ value from Mann-Whitney test. Figure adapted and reprinted with permission from (Boezio et al., 2020). License: CC BY 2.0.

In order to distinguish between a TGF- $\beta$ and BMP activation and to confirm that the $\mathrm{p}-\mathrm{Smad} 3$ antibody did not recognize other p-Smads, I immunostained zebrafish larvae for p-Smad1/5/8, the BMP-specific Smads. This difference is crucial since Alk5 has been described to participate in both the TGF- $\beta$ and BMP signaling pathways (Gronroos et al., 2012; Holtzhausen et al., 2014; Aspalter et al., 2015). As for p-Smad3, p-Smad1/5 staining was observed in the OFT region at both 24 and 75 hpf, but its staining was consistently fainter than p-Smad3 (Figure 17A, $\mathbf{A}^{\prime}$ ). Moreover, unlike for $\mathrm{p}-\mathrm{Smad} 3$, I could not appreciate any preferential localization of $\mathrm{pSmad} 1 / 5 / 8$ in the endothelium, suggesting the specificity of the two stainings (Figure 17B). These data suggest that the TGF- $\beta$ and BMP signaling are both active in the heart from the early embryonic stages. Interestingly, the activation of the Smad3/TGF- $\beta$ pathway is enriched in early OFT ECs, while alk 5 receptor expression is detected in the entire heart and all the layers of the OFT. This discrepancy suggests several regulatory steps necessary to activate the pathway only in specific subsets of cells at a specific time. Moreover, it's interesting to note how the activation of the pathway changes over time, progressively switching from ECs to SMCs, suggesting a different role in the two tissues at different developmental and adult stages. 
(Certain lines in this subsection have been quoted verbatim from Boezio et al., eLife 2020;9:e57603 for the scientific accuracy of the terms.)
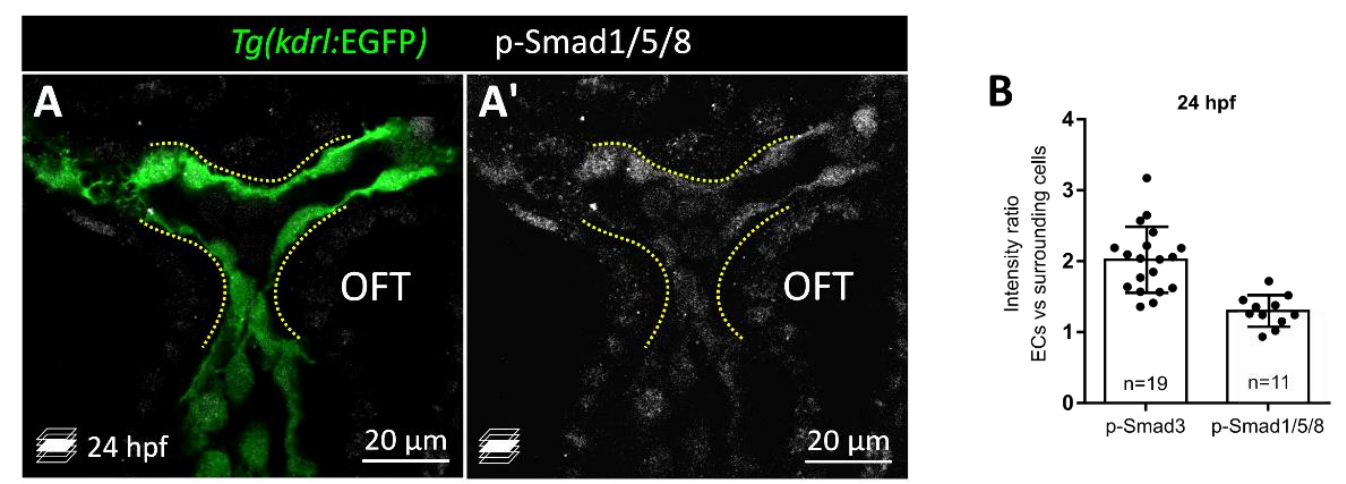

Figure 17: BMP signaling is not enriched in OFT ECs

A, A') p-Smad1/5/8 immunostaining (white) is barely detectable in the 24 hpf OFT and localized in ECs (dashed line) and surrounding cells. B) p-Smad1/5/8 immunostaining intensity is not significantly enriched in ECs, as compared with p-Smad3 at $24 \mathrm{hpf}$. Mean \pm SD; $P$ value from $t$-test. Figure adapted and reprinted with permission from (Boezio et al., 2020). License: CC BY 2.0.

\subsubsection{Mutating alk5 leads to specific defects in OFT size and expansion}

After I determined the activation of TGF- $\beta$ signaling in the developing zebrafish heart, I aimed at investigating its function. I used the CRISPR/Cas9 technique to generate mutations in alk $5 \mathrm{a}$ and alk $5 \mathrm{~b}$. I decided not to mutate the downstream effector Smad3, because of its requirement -downstream of several receptors- during earlier stages of embryonic patterning. To verify if the CRISPR/Cas9 technique induced a genomic lesion, I used high-resolution melt (HRM) analysis. I amplified a $100 \mathrm{bp}$ region around the gRNA target sequence and analyzed the melting profiles of 16 injected embryos (F0 generation), comparing them with uninjected ones. After three months, I crossed F0 animals to identify fish carrying the mutation in their germ line, and I raised F1 animals from these crosses. I subsequently genotyped the F1 adult fish and, to determine the mutant alleles, I sent the samples for sequencing.

In the F1 generation, I recovered a 2 base pairs (bp) insertion/ 6 bp deletion in alk.5a (in the ligand-binding domain; bns329 allele) and a $3 \mathrm{bp}$ insertion/ 8 bp deletion in alk.5b (in the kinase domain; bns225 allele). For alk 5 a, I also identified a 16 bp insertion/11 bp deletion allele, but after assessing the similarity between the two mutant phenotypes, I only worked with the bns 329 allele. Both alk.5a and the alk.5b mutations lead to a frameshift in the sequence, predicted to lead to the formation of truncated proteins lacking their kinase domains (Figure $18 \mathbf{A}$ ). Morphologically, single mutant larvae for alk $5 a$ or alk $5 b$ were indistinguishable from wild types (Figure 18 B-D). At larval stages, I could only observe the lack of an inflated swim bladder in alk. $5 \mathrm{~b}$ mutant larvae, which prevents them from being raised to adulthood (Figure $18 \mathbf{D}$ ). 
A

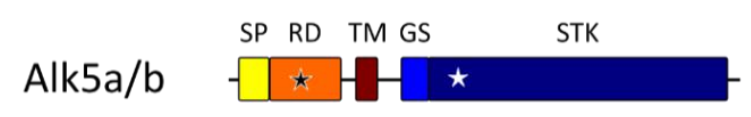

Alk5a $a^{\text {bns329 }}$

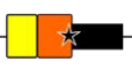

Alk5b bns225

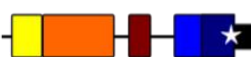

B

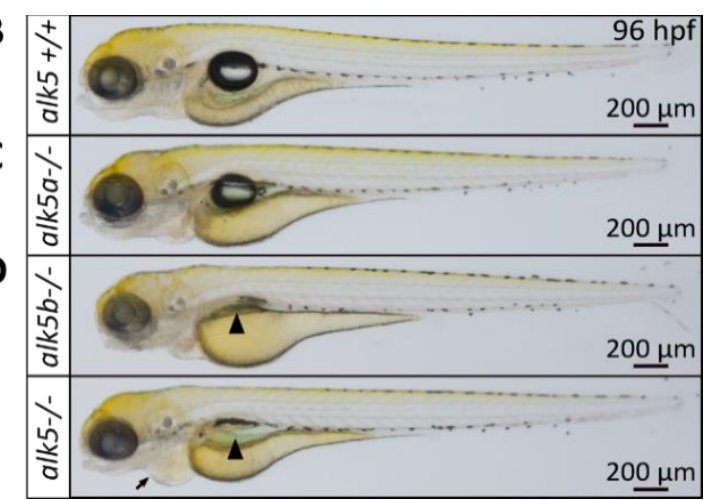

Figure 18: Generation of alk $5 a$ and alk $5 b$ mutants

A) Schematics of Alk5a/b wild-type protein, with highlighted the functional domains: SP, signaling peptide; $\mathrm{RD}$, receptor domain; TM, transmembrane domain; GS, glycine-serine rich domain; STK, serine-threonine kinase domain. Below, Alk5a bns329 and Alk5b bns225 mutant proteins. Stars refer to the CRISPR recognition sites in alk $5 a$ (white) and alk.5b (black) sequences. B-E) Brightfield images of $96 \mathrm{hpf}$ alk $5 a^{/-}$(C) or alk $5 b^{-}$(D) larvae, not exhibiting any morphological defect compared with wild types (A), except for the lack of the swim bladder inflation (D). Double mutants alk5 also exhibit severe pericardial edema (E, arrow). Figure adapted and reprinted with permission from (Boezio et al., 2020). License: CC BY 2.0.

I first hypothesized that the lack of an observable phenotype could be due to the process of transcriptional adaptation, recently described by our group (El-Brolosy et al., 2019). If this was the case, the single mutant would upregulate the transcription of the paralog gene, leading to a compensation effect that would prevent the appearance of functional defects. Therefore, I analyzed the expression levels of alk.5a and alk.5b in the two single mutants and found that both mutations caused non-sense mediated decay (NMD), leading to the degradation of the mutant RNAs (Figure 19). Notably, in neither alk $5 a^{-1-}$ nor alk $5 b^{-1-}$ mutants I observed upregulation of the paralog gene, arguing against a transcriptional adaptation phenomenon (Figure 19). Nonetheless, many functional compensation events can take place at different levels (transcriptional, post-transcriptional, translational, post-translational, etc...), so I decided to generate double mutants alk $5 a^{-/}$; alk $5 b^{-/-}$(referred to as alk. 5 mutants), to completely block the Alk5-mediated signaling.

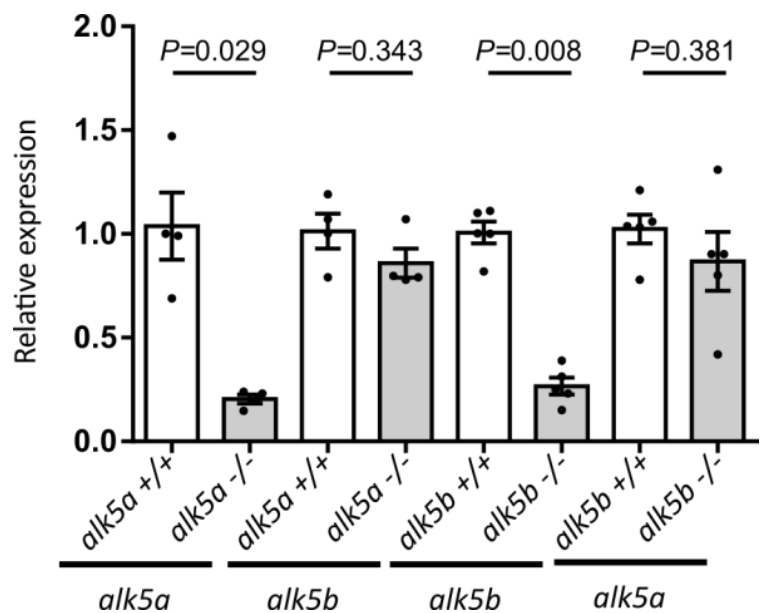

Figure 19: alk5a and alk5b mutants do not upregulate mRNA expression of the paralog gene

Relative mRNA expression by RT-PCR in single alk $5 a$ and alk $5 b$ mutant embryos, showing the degradation of the mutant mRNA without upregulation of the paralog gene. Mean \pm SD; $P$ values from Mann-Whitney tests. Figure adapted and reprinted with permission from (Boezio et al., 2020). License: CC BY 2.0. 
During early development, double mutant fish do not display any obvious developmental defects, but exhibit pericardial edema starting at $3 \mathrm{dpf}$ (Figure 18 E), suggesting defective cardiac function.
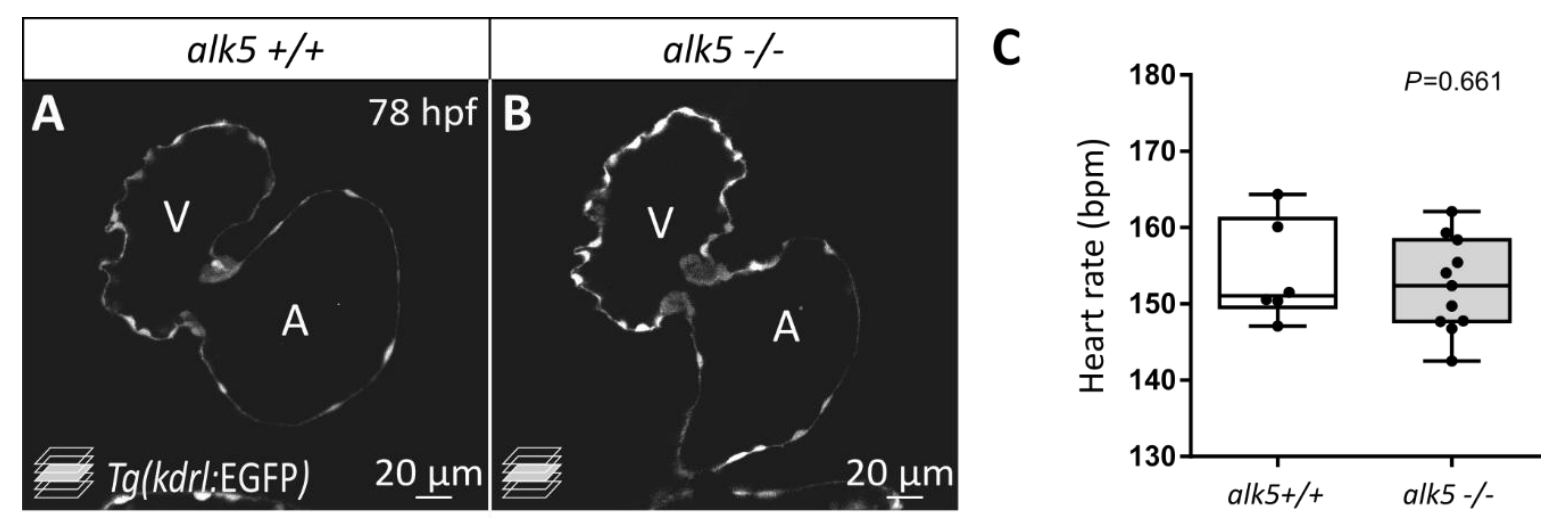

Figure 20: alk5/- larvae do not display obvious defects in cardiac chambers or cardiac rhythm A-B) 78 hpf alk.5 mutants do not exhibit obvious defects in atrial, ventricular, and valve formation. C) Heart rate quantification in $78 \mathrm{hpf}$ alke.5/+ and alk.5/-larvae. Mean \pm SD; $P$ value from Mann-Whitney test. Figure adapted and reprinted with permission from (Boezio et al., 2020). License: CC BY 2.0.

While I could not observe any defect in the morphology of the heart or the cardiac rhythm (Figure 20 A-C), I identified a very specific dilation of the OFT canal (Figure 21C, C'). Notably, an intermediate phenotype could also be observed in alk $5 a^{+/-}$;alk $5 b^{-1-}$ larvae (Figure 21 B, B'), but not in alk. $5 a^{-/}$; alk $5 b^{+/-}$(Figure 21A). This result indicates that the homozygous mutation in alk $5 b$ is crucial for the appearance of the phenotype, which is unmasked by losing only one copy of alk.5a. alk $5 a^{-/}$; alk.5 $b^{+/}$animals survived to adulthood and were fertile. These animals were used to generate the alk. $5 \%$ embryos for most of the subsequent experiments. Mutant fish were compared with wild types deriving from parents siblings of the alk.5\% parents. This setting was established to have a higher number of homozygous embryos (25\%), but to be sure that the comparisons were done within the same genetic background.

Then, I analyzed alk.5 mutant embryonic hearts from early developmental stages, to identify the onset of the phenotype. At early developmental stages ( $30 \mathrm{hpf}$ ), the diameter of alk 5 mutant OFT was comparable with wild types (Figure 22A-C), while the defects started appearing at 54 hpf (Figure 22D-F) and became more severe at 78 hpf (Figure 22G-H). At 78 hpf, the mutant OFT dilation affected OFT expansion during cardiac contractions (a parameter similar to ventricular ejection fraction), which was more than twice as the wild-type (Figure 22I). At this stage, the OFT dilation also caused regurgitation of the blood into the ventricle, failing to pump it into the vascular network. The impaired blood flow in the rest of the body became more severe with time, leading eventually to the death of the larvae by $6 \mathrm{dpf}$. 


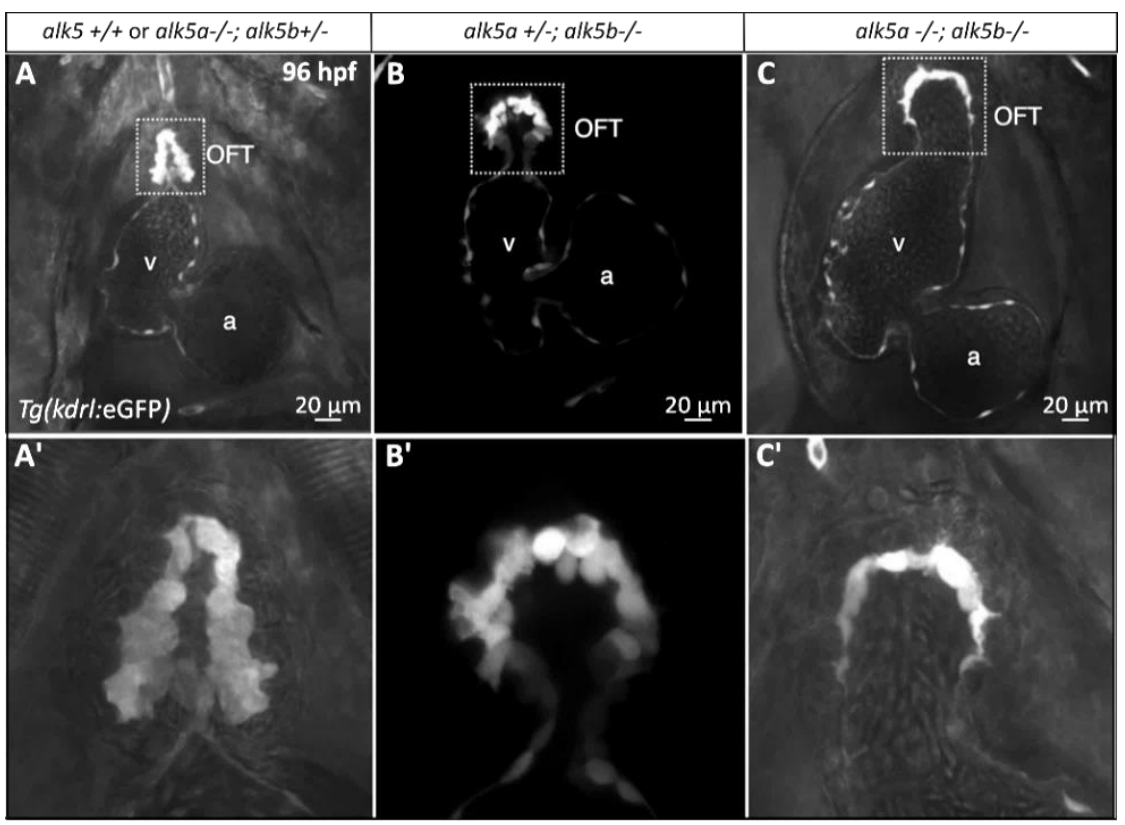

Figure 21: alk5 double mutant larvae exhibit a severe OFT dilation

A-C') 96 hpf alk. 5 double mutants exhibit severe OFT dilation (C). While alk $5 a^{+/-;}$alk $5 b^{-/}$(B) display an intermediate OFT phenotype, alk.5 $5 a^{-/}$;alk $5 b^{+/-}$(A) are indistinguishable from wild types. Boxed areas are shown in A', B', C'.

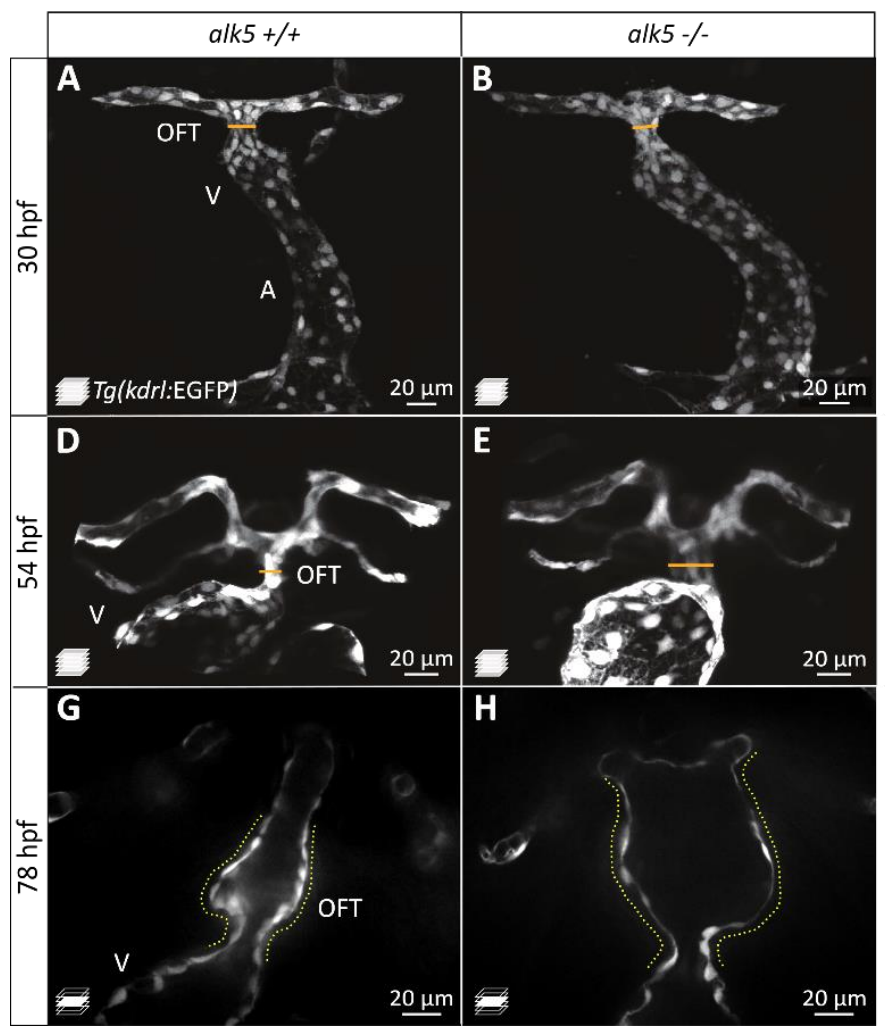

C

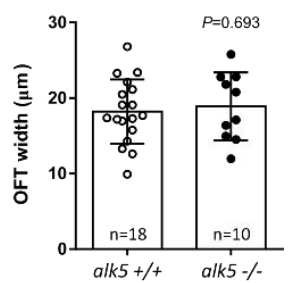

$\mathbf{F}$
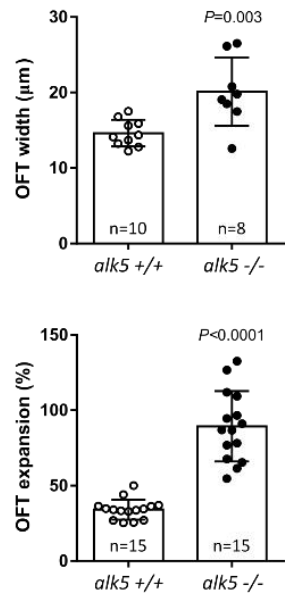

Figure 22: alk5 mutant larvae progressively exhibit OFT enlargement and increased expansion A-F) Confocal images and quantifications of $30 \mathrm{hpf}$ (A-C) and $54 \mathrm{hpf}$ (D-F) alk 5 mutant hearts. At 30 hpf, mutant OFTs exhibit no morphological defects (A, B) or width difference (C). At 54 hpf, some of the alk.5 mutant larvae display an increase in OFT width. G-I) Frames of $78 \mathrm{hpf}$ beating hearts and quantification (I), showing severe alk $5 \%$ OFT expansion. Mean \pm SD; $P$ values from $t$-tests. Figure adapted and reprinted with permission from (Boezio et al., 2020). License: CC BY 2.0. 
In wild-type zebrafish, the OFT is initially connected to the first pair of aortic arches until 6065 hpf. From this stage onwards, one could start observing the formation of a single vessel originating from the OFT, the ventral aorta (VA), generated by a poorly understood process (Figure 23A). alk 5 mutants fail to form a single VA and maintain two independent connections from the OFT to the aortic arches (Figure 23B). Notably, the pattern of the aortic arches is not affected by the alk.5 mutations, and also the diameter of the dorsal aorta is comparable with wild types at both 56 and $96 \mathrm{hpf}$ (Figure 23C). These data suggest a high specificity of the phenotype, making alk.5 mutants an important model to study OFT development without complications arising from other compromised processes.
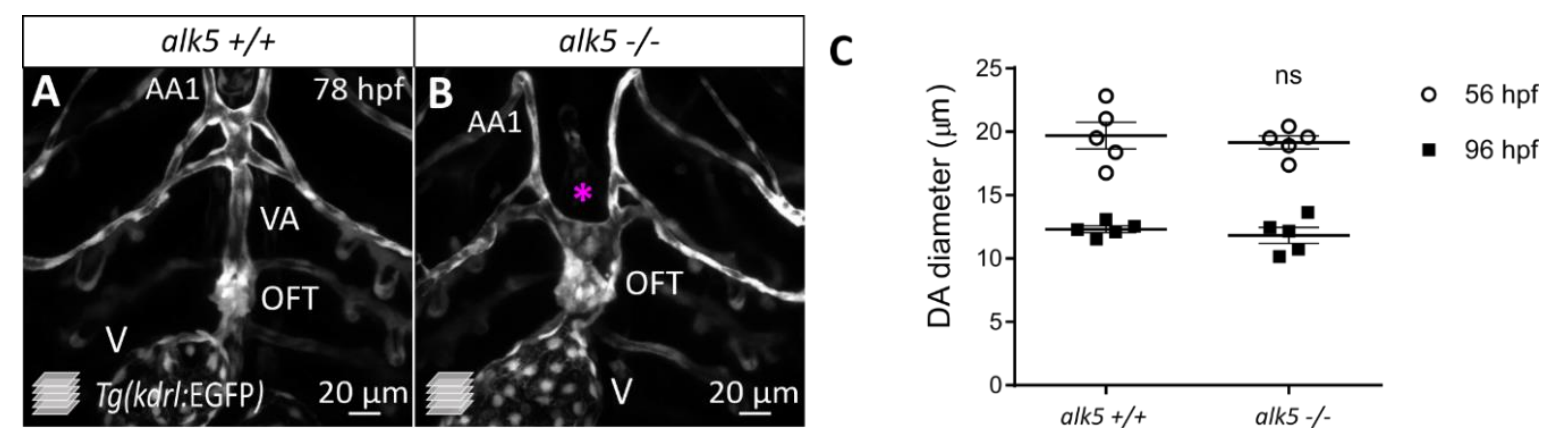

Figure 23: alk5 /- larvae fail to form the ventral artery, but exhibit no defects in other blood vessels A-B) Confocal images of $78 \mathrm{hpf}$ wild-type and alk.5 mutant larvae. alk.5\% larvae exhibit two distinct connections to the aortic arches (AA), failing to form a single ventral artery (VA) as the wild types (A). C) No difference in the dorsal aorta (DA) diameter is observed at 56 or 96 hpf. Figure adapted and reprinted with permission from (Boezio et al., 2020). License: CC BY 2.0.

The OFT dilation and expansion observed in the mutant are reminiscent of the thoracic aortic aneurysm (TAA) formation often observed in TGFBR1 human patients (Loeys et al., 2006; Matyas et al., 2006; Takeda et al., 2018; Camerota et al., 2019). On rare occasions, the aneurysms lead to a dissection of the aorta due to the rupture of the endothelial layer. When I followed the progression of the alk 5 mutant phenotype, I observed ruptures in the OFT endothelium at $96 \mathrm{hpf}$ in $\sim 5 \%$ of the larvae (Figure 24A, $\mathbf{A}^{\prime}$ ). I confirmed the rupture of the layer and the leakage of the fluids with the injection of fluorescent dextran into the circulation. 10 minutes after injection, in $63.4 \%$ of alk 5 mutant larvae $(n=11)$ I observed dextran accumulation outside of the endothelial layer, between ECs and SMCs, and in the extracellular space among SMCs (Figure 23C). This phenotype was never observed in wild-type animals (Figure 23B). (Certain lines in this subsection have been quoted verbatim from Boezio et al., eLife 2020;9:e57603 for the scientific accuracy of the terms.) 

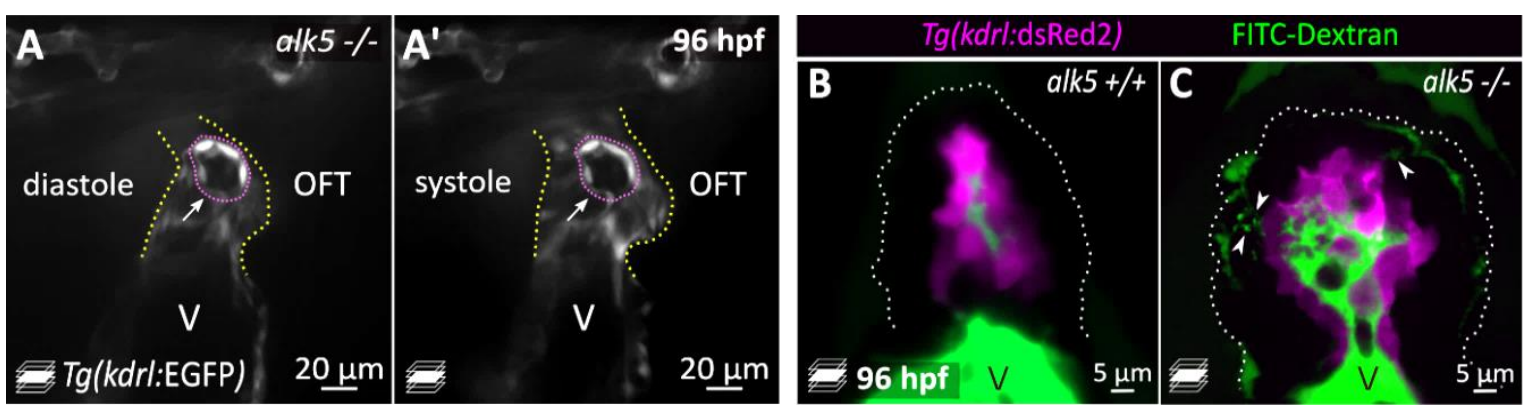

Figure 24: The alk5 mutant OFT phenotype occasionally results in endothelial ruptures

A, A') Frames of beating heart confocal movies during ventricular diastole (A) and systole (A'). Occasionally, 96 hpf alk 5 mutants exhibit ruptures in the OFT endothelial layer. B, C) 7/11 alk 5 mutant and 0/10 alk $5^{+/+}$ larvae exhibit dextran (green) accumulation between ECs and SMCs (arrowheads). Figure adapted and reprinted with permission from (Boezio et al., 2020). License: CC BY 2.0.

\subsubsection{Loss of Alk5 affects the Smad3 signaling axis}

In human patients, the molecular effect of the TGFBR1 mutations responsible for the development of TAAs is still very controversial. Interestingly, in both human patients and mouse models, despite the loss-of-function nature of the mutation, hyperactivation of the downstream pathway was often observed, identified by upregulation of $\mathrm{p}$-Smad3 staining and downstream genes (Jones et al., 2009; F. Lin and Yang, 2010; Renard et al., 2013). In contrast with these findings, the administration of a TGF- $\beta$ inhibitor such as Losartan does not appear to be effective in the treatment of the disease and, often, it even exacerbates the defects (Jones et al., 2009; F. Lin and Yang, 2010; Gillis et al., 2013). Therefore, it is important to develop a model of aneurysm formation that could be analyzed from earlier stages, before the appearance of the morphological defects. After establishing alk.5 as a potential model, I aimed at taking advantage of it to better understand the molecular mechanism downstream of Alk5 in the OFT. I previously identified upregulation of the Smad3 signaling axis in OFT ECs (Figure 16C, D), thus I assessed the activation of the signaling in alk 5 mutants. I performed p-Smad3 immunostaining in $24 \mathrm{hpf}$ wild-type and mutant hearts, prior to the appearance of the OFT morphological phenotype. At this stage, I could observe a significant downregulation of the signal, particularly in OFT ECs but -to a lower extent- also in the cells surrounding the endothelium (Figure 25A-C). To my surprise, when I performed the same experiment at 75 hpf, a stage when the OFT dilation is already very pronounced, I observed and quantified a very obvious increase in the p-Smad3 immunostaining, particularly in SMCs (Figure 25D-F). This last result is in line with the mammalian data, which also focused on samples obtained when the aortic dilation was already evident. 

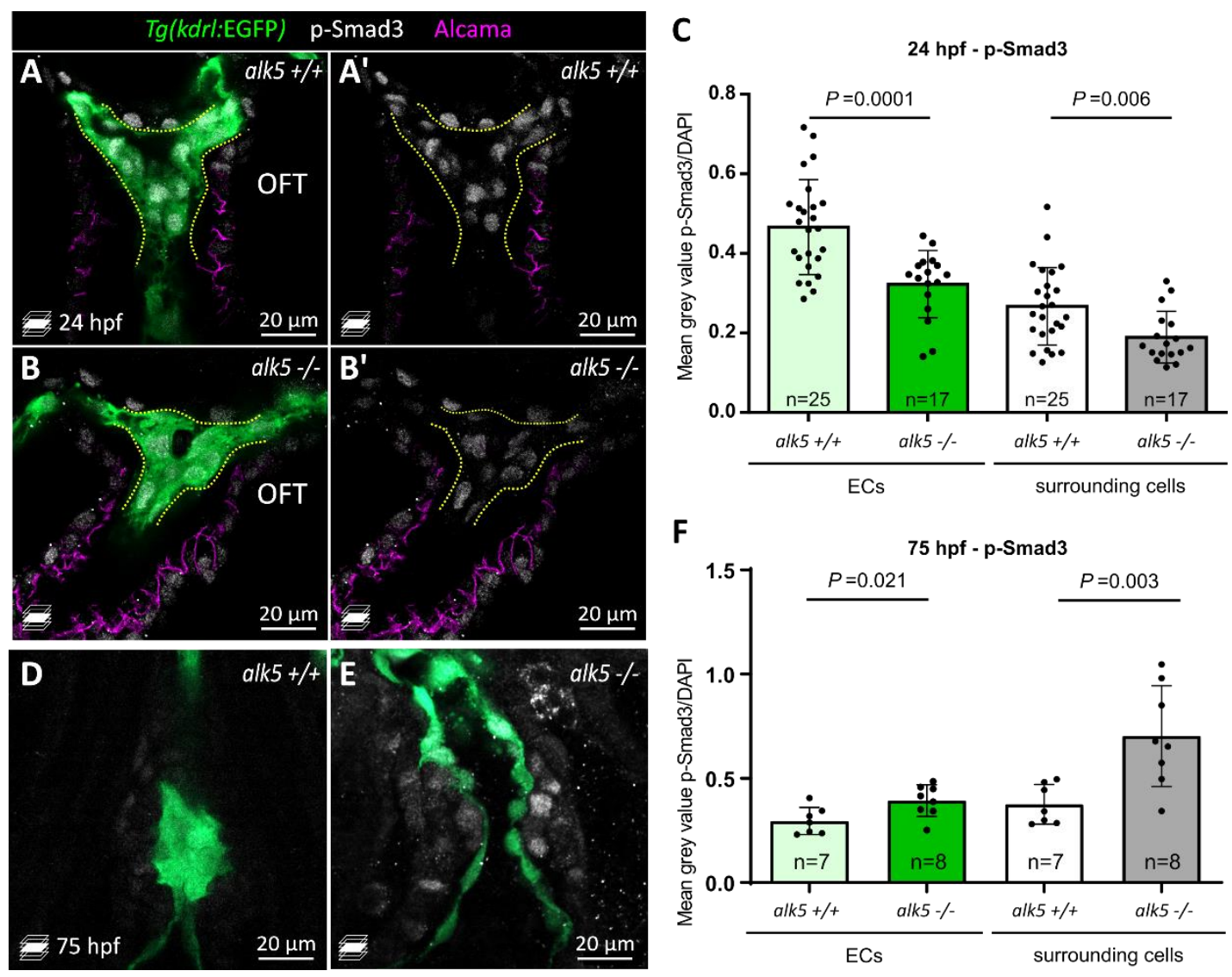

Figure 25: alk5 mutation has distinct effects on Smad3 signaling at different developmental stages. A-C) Confocal pictures and quantification of p-Smad3 immunostaining intensity in $24 \mathrm{hpf}$ alk.5 $5^{+/+}$(A) and alk.5\% (B) embryos. P-Smad3 staining relative intensity is reduced in alk.5\% OFTs, especially in ECs (green). Mean \pm SD; $P$ value from $t$-tests. D-F) Confocal pictures and quantification of $\mathrm{p}$-Smad3 immunostaining intensity in $75 \mathrm{hpf}$ wild-type (D) and alk.5\% (E) larvae. P-Smad3 staining relative intensity is increased in alk.51- OFTs, especially in SMCs (grey; surrounding cells). Mean \pm SD; P value from Mann-Whitney tests. Figure adapted and reprinted with permission from (Boezio et al., 2020). License: CC BY 2.0.

With the aim of understanding if the downregulation or the upregulation of the Smad3 signaling was responsible for the OFT defect, I took advantage of a specific and potent Smad3 inhibitor (SIS3) Jinnin et al., 2006; Dogra et al., 2017). I chose a concentration (1.5 $\mu \mathrm{M})$, which preserved the heart morphology and did not affect other vascular beds (similar to alk.5 mutants). Then, I treated the embryos from $36 \mathrm{hpf}$ to $75 \mathrm{hpf}$, a crucial window for OFT development (Figure 26). Using this concentration of SIS3, I could not observe any morphological defect in OFT in 75 hpf wild-type larvae, but surprisingly the OFT expanded significantly more than in DMSO treated ones (Figure 26E). Besides, in SIS3-treated alk $5^{+/-}$fish I also observed the lack of the VA, a phenotype usually observed only in homozygous larvae (Figure 26A, B). The treatment with SIS3 also exacerbated the phenotype of alk $5^{-/}$fish, leading to a more severe expansion of their OFT compared to untreated animals (Figure 26C, D). 


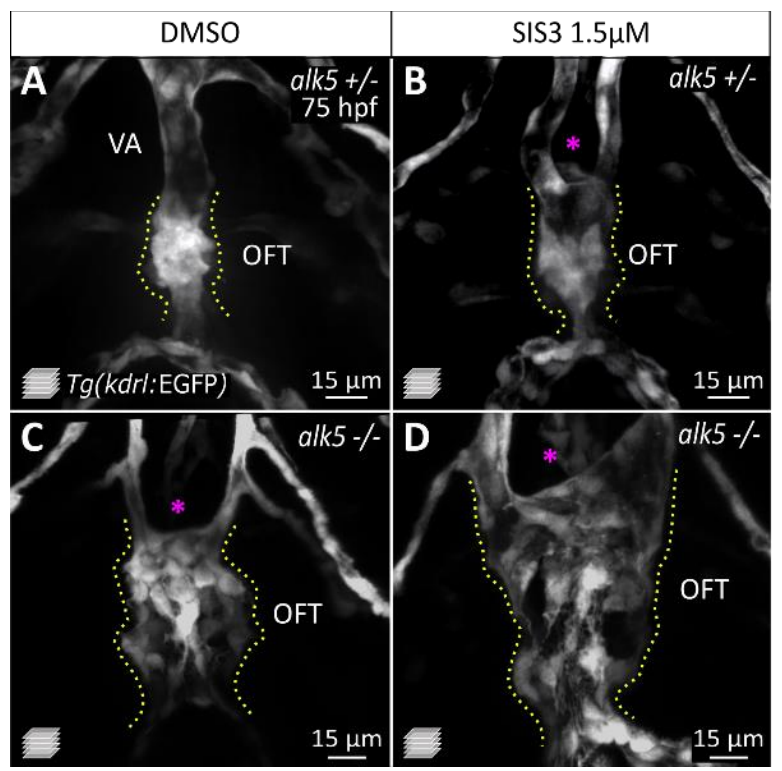

$\mathbf{E}$

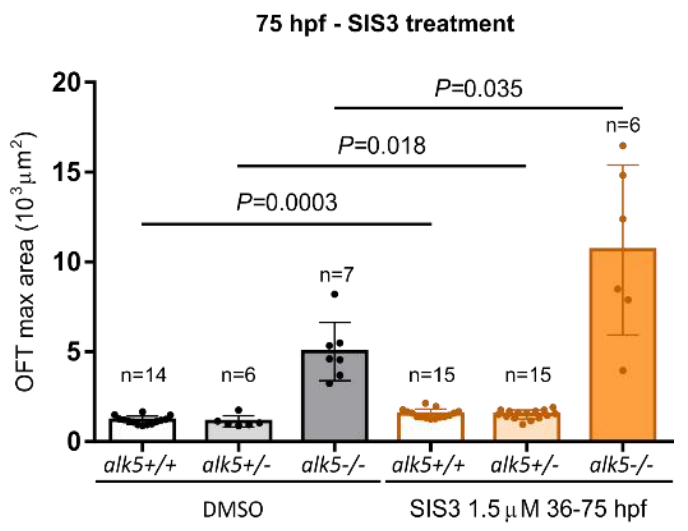

Figure 26: The Smad3 inhibitor SIS3 induces mild OFT defects in alk $5^{+/-}$and exacerbates OFT dilation in alk $5^{-/}$larvae.

A-D) Confocal images of $75 \mathrm{hpf}$ alk $5^{+/-}$(A, B) and alk 5 - (C, D) OFTs in larvae treated with DMSO (A, C) or SIS3 (B, D). alk $5^{+/-}$larvae (B) fail to form the VA (asterisk) when Smad3 signaling is inhibited, unlike the untreated ones (A). E) Quantification of OFT expansion of $75 \mathrm{hpf}$ larvae treated with DMSO or SIS3. Mean \pm SD; $P$ values from Mann-Whitney tests comparing the selected columns. Figure adapted and reprinted with permission from (Boezio et al., 2020). License: CC BY 2.0.

With these data, I can hypothesize that the initial cause of the OFT phenotype relies in reduced activation of the signaling, as suggested by the early $\mathrm{p}-\mathrm{Smad} 3$ downregulation and the SIS3 treatments. Later on, the appearance of the morphological phenotype likely activates compensatory signaling, which leads to an independent increase in p-Smad3 staining. These data can open new future avenues of research directed to dig deeper into the underlying cause of TAAs in mammalian models. (Certain lines in this subsection have been quoted verbatim from Boezio et al., eLife 2020;9:e57603 for the scientific accuracy of the terms.)

\subsubsection{Alk5 inhibitor recapitulates the morphological phenotype of alk5 mutants}

Several chemical inhibitors of the TGF- $\beta$ signaling have been used in zebrafish, particularly to impair the Nodal signaling during gastrulation, or to impair cardiac regeneration after myocardial infarction. The most commonly used inhibitors are SB431542 or SB505124 (Scherz et al., 2008; Chablais and Jazwinska, 2012; van Boxtel et al., 2015). To complement our data with an Alk5 inhibitor, I aimed to recapitulate the alk.5 phenotype with chemical compounds. This tool would be useful to save time and animals for a preliminary screening of the phenotypes. Given the possibility of treating different transgenic lines with the compounds, I could first identify the promising processes, before growing the double mutants in a specific transgenic background. Moreover, once identified as a compound that phenocopies alk.5 
mutants, this versatile pharmacological treatment can be used for several other purposes in future studies.

Thus, I treated wild-type larvae with SB431542 or SB505124 up to $50 \mu \mathrm{M}$ from 24 to $78 \mathrm{hpf}$, but I could not identify any OFT defect (data not shown). In a last attempt to identify a specific Alk5 inhibitor, I used E-616452 (Gellibert et al., 2004), a new compound described to be a potent and selective Alk5 inhibitor, but never used in zebrafish before. I tested different concentrations and confirmed its effectiveness in zebrafish. Already at $10 \mu \mathrm{M}$ I could observe toxicity of the inhibitor when used from 24-75 hpf. Thus, I lowered the concentration and identified $2.5 \mu \mathrm{M}$ as the lowest effective dose, which did not cause any developmental or morphological defect. Surprisingly, when treating the embryos from 24-75 hpf, I could recapitulate the mutant OFT phenotype, with the complete absence of off-target effects or any other defect in the cardiovascular system. I aimed at narrowing the time-window to find the short possible treatment which would lead to the alk.5 mutant phenotype. As a result, I found that 36-54 hpf was the short most effective treatment (Figure 27).

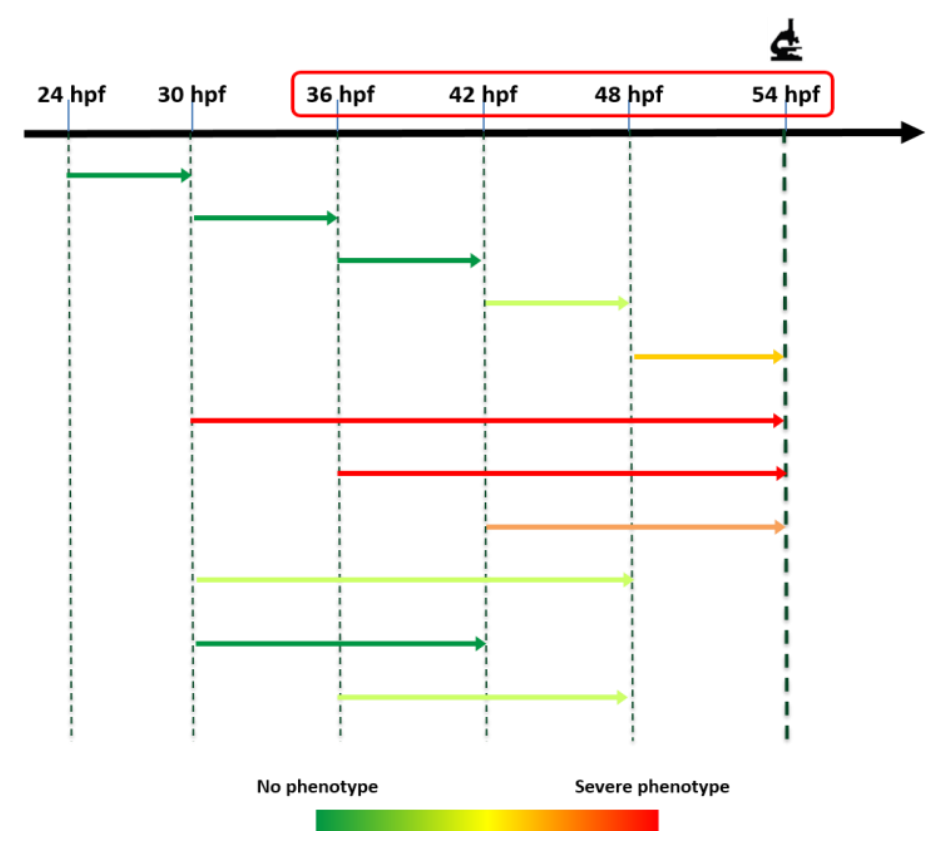

Figure 27: The alk5/-OFT phenotype can be recapitulated by 36-54 hpf treatment with E-616452 Treatment with $2.5 \mu \mathrm{M}$ E-616452 leads to different severity of the OFT phenotype at different developmental stages (red, severe; green, no phenotype). The shortest effective time-frame was 36-54 hpf.

With this treatment, I confirmed the OFT dilation and severe OFT expansion both at 54 and 78 hpf (Figure 28A-F). These results provide a new important pharmacological tool able to mimic the loss of Alk5 and recapitulate the specific OFT phenotype observed in alk. 5 mutants. (Certain lines in this subsection have been quoted verbatim from Boezio et al., eLife 2020;9:e57603 for the scientific accuracy of the terms.) 
A

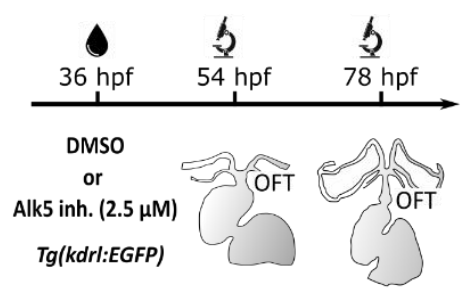

$\mathbf{F}$

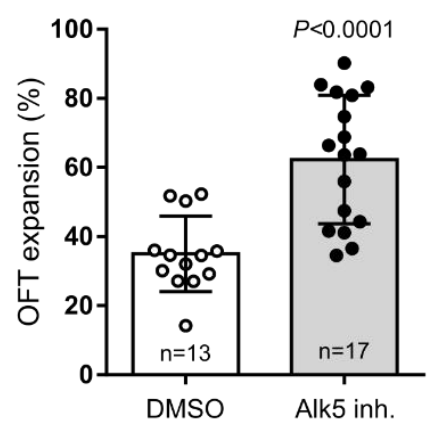

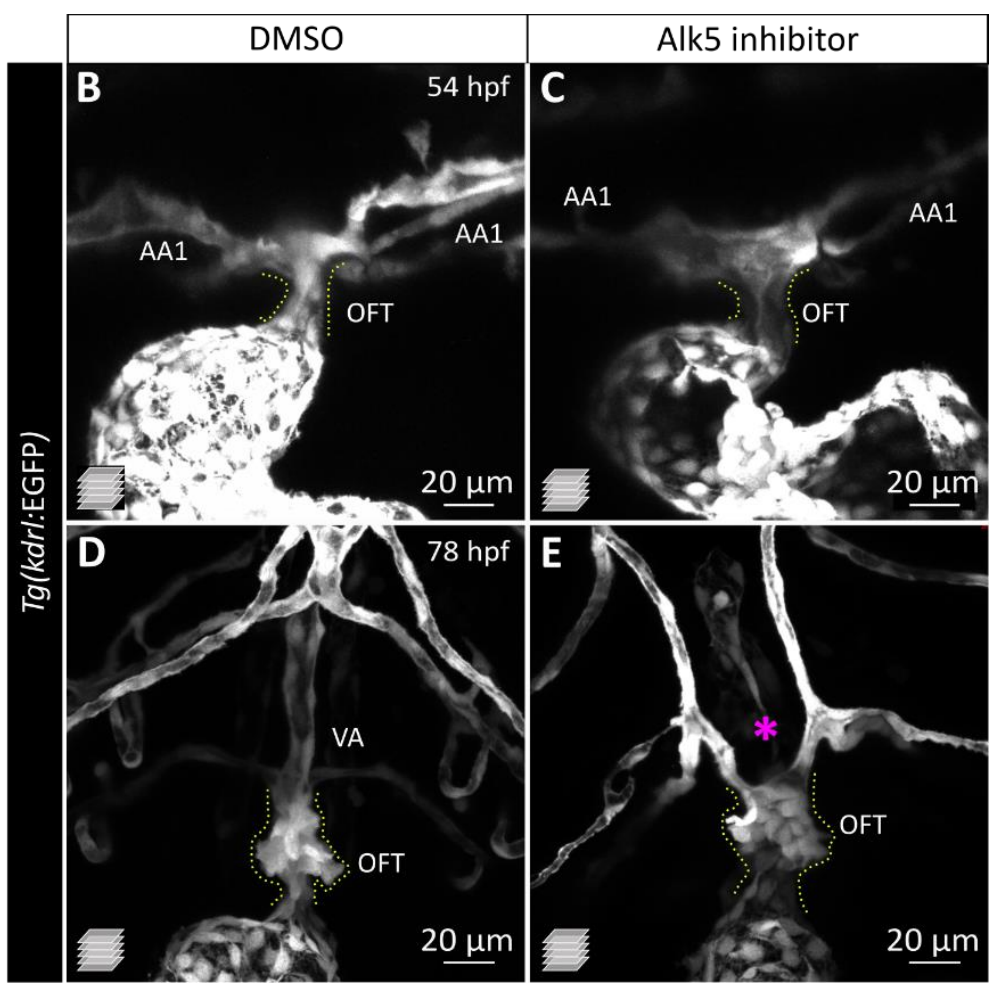

Figure 28: Treatment with E-616452 increases OFT expansion in wild-type larvae.

A) Schematic of the inhibitor treatment. B-E) Confocal pictures of 54 (B, $C ; n=11$ per genotype) and 78 (D, $\mathrm{n}=13 ; \mathrm{E}, \mathrm{n}=17$ ) hpf OFTs in larvae treated with DMSO or E-616452. F) Quantification of OFT expansion at $78 \mathrm{hpf}$ in larvae treated with DMSO or E-616452. Mean \pm SD; $P$ value from $t$-test. Figure adapted and reprinted with permission from (Boezio et al., 2020). License: CC BY 2.0.

\subsubsection{Alk5 limits OFT EC proliferation from early developmental stages}

Most of the literature dealing with defects downstream of the loss of TGFBR1 focuses on its importance in SMCs (Perrucci et al., 2017). Nonetheless, my previous results identified an earlier activation of the downstream signaling in OFT ECs, when SMCs are not yet present around the OFT canal (Figure 16A, D; Figure 25C). In fact, smooth muscle coverage appears around 56 hpf in zebrafish (Grimes and Kirby, 2009; Zhou et al., 2011; Jahangiri et al., 2016; Felker et al., 2018). Therefore, I decided to first assess the endothelial phenotypes and, given the increased size of the OFT in alk.5 mutants, I wondered if this defect could be explained by a higher number of ECs. The OFT, to transition from a simple ventricular constriction to a pear-shaped structure, doubles its cell between 36 (approximately 20 cells) and $72 \mathrm{hpf}$ (more than $40 \mathrm{ECs})$. At $36 \mathrm{hpf}$, I could not observe any difference in EC number between alk $5^{+/+}$ and alk $5 \%$ OFT, but at $72 \mathrm{hpf}$ the mutant alk 5 OFT was composed of more than $80 \mathrm{ECs}$, twice as observed in the wild types (Figure 29). 


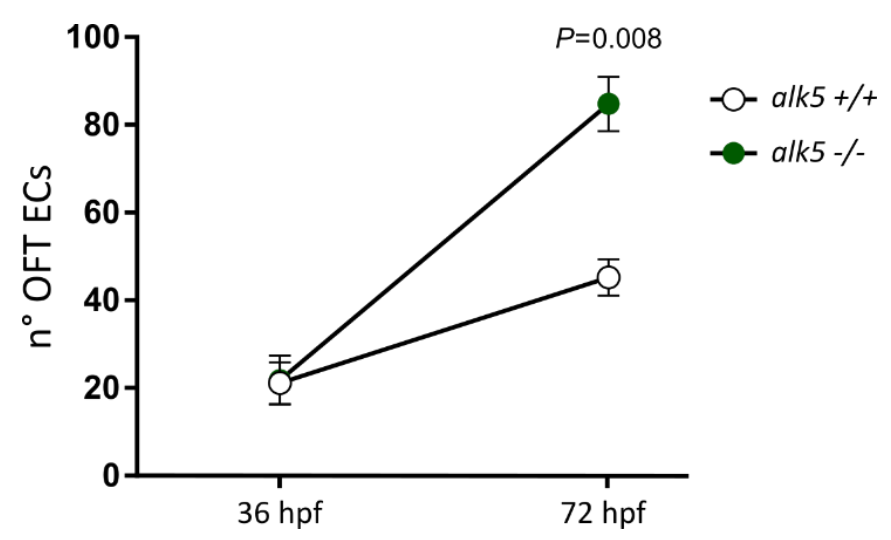

Figure 29: Alk5 regulates EC number during OFT development. At $36 \mathrm{hpf}$, alk $5^{+/+}$and alk $5 \%$ OFTs are composed by a similar number of ECs (approximately 20; $\mathrm{n}=11$ per genotype). $72 \mathrm{hpf}$ alk $5 \%$ OFTs have twice the number of ECs as wild types ( $\mathrm{n}=6$ per genotype). Mean \pm SD; $P$ value from Mann-Whitney test. Figure adapted and reprinted with permission from (Boezio et al., 2020). License: CC BY 2.0.

Several reasons could account for this dramatic increase in ECs, ranging from increase proliferation to impaired migration, to reduced cell death. I decided to first analyze the EC proliferation process, taking advantage of EdU labeling (Figure 30). 5-Ethynyl-2'-deoxyuridine $(\mathrm{EdU})$ is a nucleotide analog, which is incorporated in the DNA while cells are entering the S phase of the cell cycle. Between 48 and $72 \mathrm{hpf}$, I observed a significant increase in the percentage of $\mathrm{EdU}^{+}$ECs in the alk5 mutant OFT, which could explain the increased cell number observed at $72 \mathrm{hpf}$ (Figure 30D-F). To my surprise, I found a slight but significant increase in the number of alk $5^{-1-} \mathrm{EdU}^{+} \mathrm{ECs}$ when I treated the embryos with EdU from 24 to $36 \mathrm{hpf}$ (Figure 30A-C). At $36 \mathrm{hpf}$, the total number of ECs in alk.5 mutants was not changed compared to wild types, but ECs appear to be already more prone to proliferate. This result suggests a defect at an earlier time point than previously expected, when the mutant OFT appears morphologically still indistinguishable from wild-type. (Certain lines in this subsection have been quoted verbatim from Boezio et al., eLife 2020;9:e57603 for the scientific accuracy of the terms.)

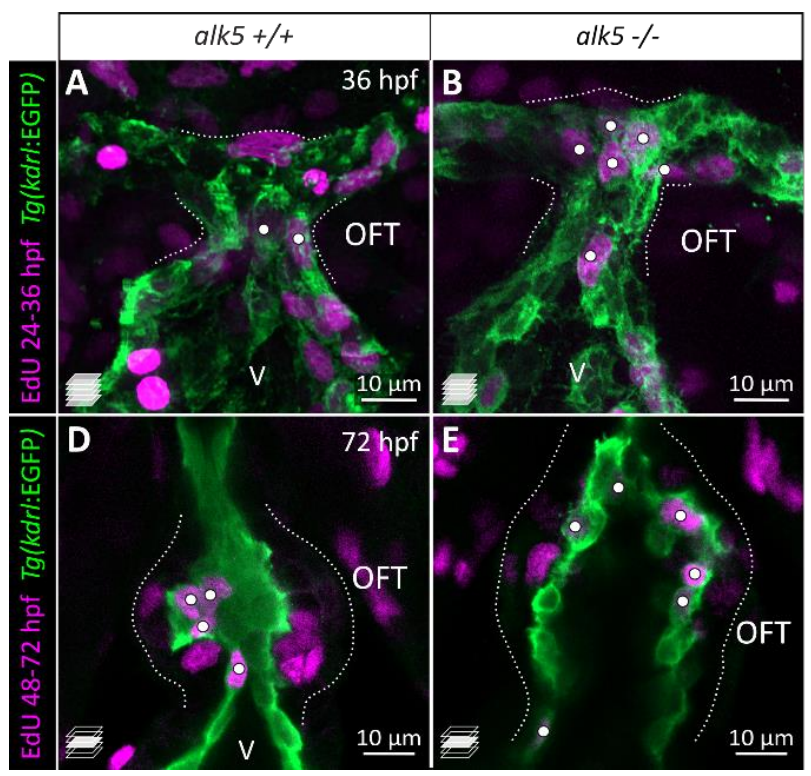

C

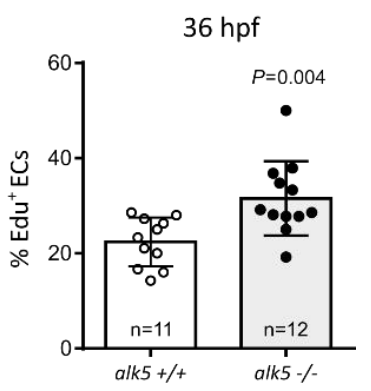

$\mathbf{F}$

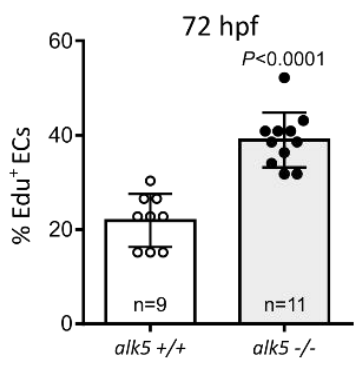

Figure 30: Alk5 restricts EC proliferation in the developing OFT. 
Figure 30: Alk5 restricts EC proliferation in the developing OFT. (previous page)

A-C) Confocal projections and quantification of EdU ${ }^{+} \mathrm{ECs}$ (white dots) in $36 \mathrm{hpf}$ alk.5 $5^{+/+}$(A) and alk.5\% (B)

OFTs. D-F) Confocal images and quantification of EdU $\mathrm{ECs}^{+}$(white dots) in $72 \mathrm{hpf}$ alk.5 $5^{+/+}$(D) and

alk $5 \%$ (E) OFTs. Mean \pm SD; $P$ values from $t$-tests. Figure adapted and reprinted with permission from (Boezio et al., 2020). License: CC BY 2.0.

\subsubsection{Alk5 promotes the formation of the ventral artery and EC displacement from the OFT}

Alongside proliferation, migration of ECs has been previously involved in the patterning of the blood vessels, especially showing that ECs from the aortic arches move against the blood flow towards the heart (Rochon et al., 2016). Therefore, the lack of VA in alk.5 mutants could suggest a potential impairment in EC displacement. To investigate this process in absence of Alk5 activity, I took advantage of the Alk5 inhibitor previously described in section 4.1.4. Combining this tool with the $\operatorname{Tg}(f l i 1 a: G a l 4 F F) ; \operatorname{Tg}(U A S:$ Kaede) transgenic line, I aimed at photoconverting ECs (which switch from green to red fluorescence when excited with $405 \mathrm{~nm}$ wavelengths) in the region of the OFT. Subsequently, I followed the photoconverted cells to understand their behavior in contributing to the patterning of the vascular network. In wild-type fish, I first aimed at identifying the population of ECs giving rise to the VA, which was not previously described. Thus, I first photoconverted ECs at $54 \mathrm{hpf}$ in the proximal region of the OFT close to the bulbo-ventricular (BV) valve (Figure 31A, B). At 74 hpf, 20 hours after the photoconversion, these distal ECs were always found located in the OFT, close to the photoconversion site (Figure 31C). Notably, I could observe that -while I photoconverted only one/two cells per embryo- I found several photoconverted cells in the $74 \mathrm{hpf}$ OFT, suggesting their active proliferation.

Subsequently, I photoconverted ECs in the most distal region of the OFT between the two aortic arches (Figure 31D, E). At $74 \mathrm{hpf}$, distal ECs were invariably found in the VA and never in the OFT (Figure 31F). After identifying in the distal OFT ECs the population that majorly contributes to the VA formation, I investigated the localization of distal photoconverted ECs in Alk5 inhibitor-treated larvae. Distal photoconverted ECs lacking Alk5 signaling preferentially remained in the OFT until $74 \mathrm{hpf}$, unlike the control (Figure 31G-H). These cells were found statistically closer to the site of photoconversion compared with ECs in control larvae at $74 \mathrm{hpf}$ (Figure 31I). To observe this process in a dynamic manner and at a high resolution, I recorded time-lapse movies from 56 to $74 \mathrm{hpf}$, following the photoconversion at 54 hpf. With this experiment, I could observe live ECs forming the VA. While the structure of the OFT grows in size, ECs devoid of Alk 5 activity were close to their original position for the entire imaging time. In contrast, ECs in control larvae seemed to extend rostral and sent out protrusions reminiscent of migrating cells. 
A

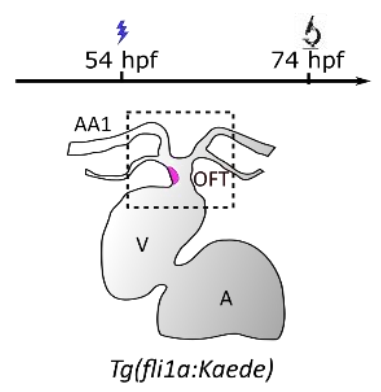

D
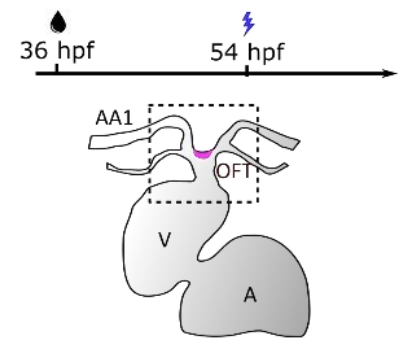

$\operatorname{Tg}($ fli1a:Kaede)

I

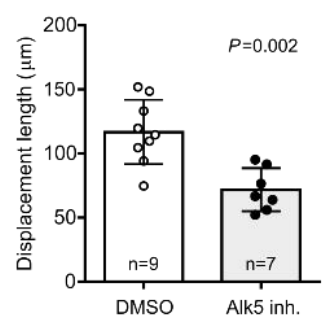

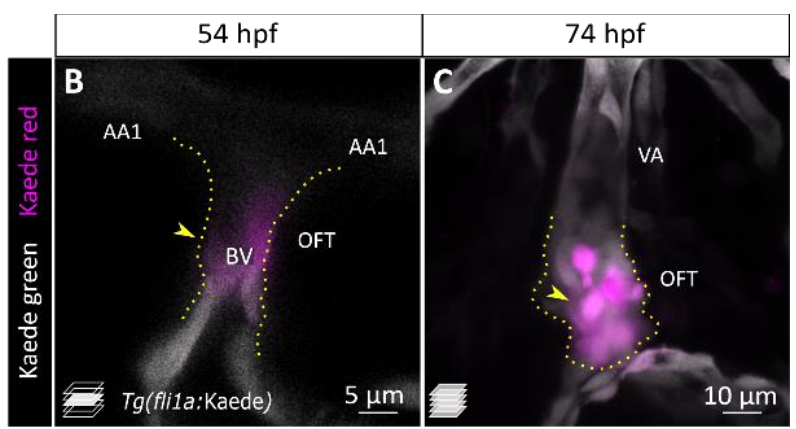

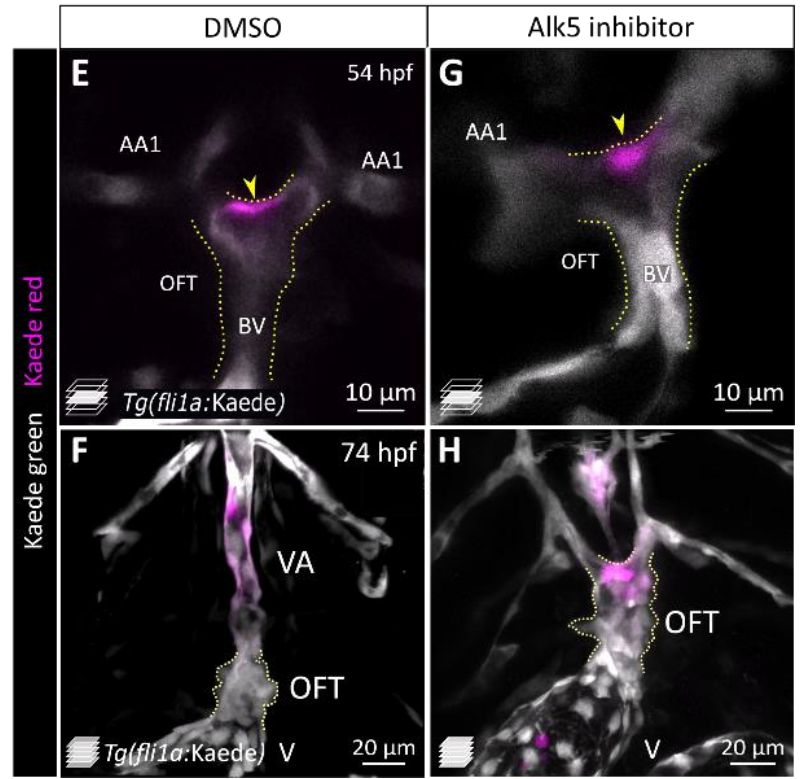

Figure 31: The displacement of distal OFT ECs into the VA is affected when Alk5 function is impaired.

A-C) Proximal OFT ECs photoconverted (magenta) at $54 \mathrm{hpf}$ (A, B) remain in the OFT until $74 \mathrm{hpf}$ (C). Yellow arrow, photoconverted EC. D-F) Distal OFT ECs photoconverted (magenta) at $54 \mathrm{hpf}$ (D, E) are invariably found in the VA at $74 \mathrm{hpf}(\mathrm{F})$. G-I) Photoconverted distal OFT ECs (G) remain closer to the OFT in 74 hpf larvae when treated with Alk5 inhibitor. Mean \pm SD; $P$ value from Mann-Whitney test. Figure adapted and reprinted with permission from (Boezio et al., 2020). License: CC BY 2.0.

Altogether, these data suggest that Alk5 promotes EC displacement to form the ventral artery. If this displacement is active and due to migration or passively caused by the rearrangement of the structure and by the proliferation of neighboring cells remains to be determined and requires further investigation. (Certain lines in this subsection have been quoted verbatim from Boezio et al., eLife 2020;9:e57603 for the scientific accuracy of the terms.)

\subsubsection{SMC proliferation and organization are regulated by Alk5 to promote the formation of the OFT wall}

During development, the OFT is progressively covered by SMC precursors, which will give rise to its external wall. The formation of a strong muscular wall, in mammals as well as in fish, is what distinguishes the great arteries that exit the heart from the rest of the vessels, as it is required to buffer the high blood pressure coming from the ventricle(s). In order to assess the 
formation of the SMC wall, I first used the Tg(pdgfrb:eGFP) transgenic line, which labels both pericytes and SMCs (collectively referred to as mural cells) and is expressed in all the cells surrounding the OFT. As this line also marks ECs, to identify SMCs, I used it in combination with EC-specific transgenic lines, as Tg(kdrl:EGFP). I observed that the OFT in 75 hpf wildtype larvae is covered by approximately $90 \mathrm{pdgfrb}^{+}$mural cells, organized in 2 or 3 layers, and positioned close to each other, separated by a small extracellular space (Figure $\mathbf{3 2 A}, \mathbf{A}^{\mathbf{\prime}}$ ). In alk.5 mutants, SMCs number was markedly reduced (-27\%) compared with wild types (Figure 32C). This reduction in the number of alk $5^{\%} \mathrm{SMCs}$ is likely due to a defect in their proliferation, as confirmed by a 53\% decrease in EdU incorporation between 48 and $72 \mathrm{hpf}$ (Figure 32D). Also, the remaining alk. $5 \%$ smooth muscle cells displayed a less compact arrangement, with wider extracellular spaces and mostly positioned in 1 or 2 disorganized layers (Figure 32B, B').
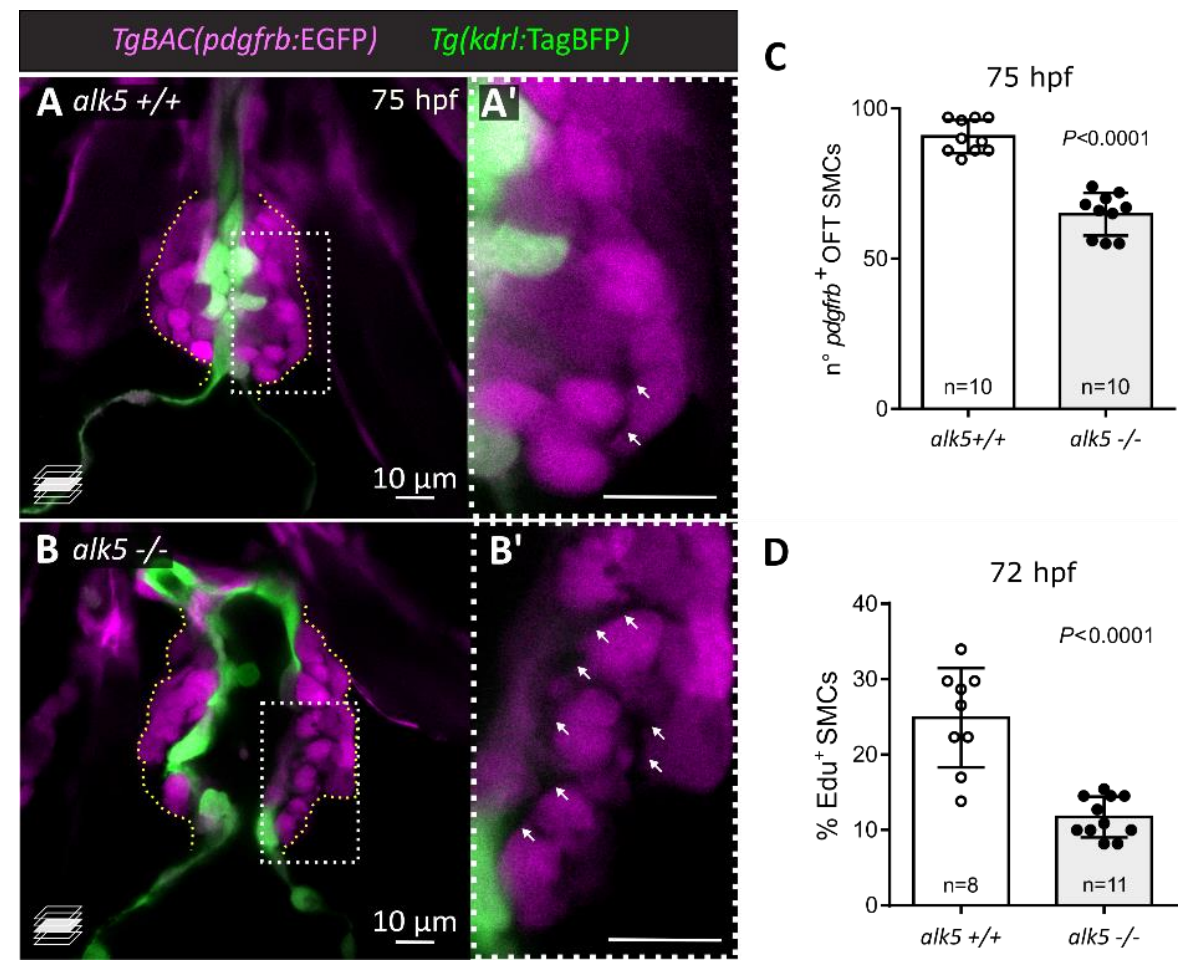

Figure 32: Alk5 regulates SMCs number and proliferation in the OFT.

A-B') Confocal images of $75 \mathrm{hpf}$ alk $5^{+/+}$(A) and alk $5 \%$ (B) OFTs. SMCs are shown in magenta; boxed areas are shown in A' and B'. Arrows point to the extracellular space between SMCs. C) Quantification of $p d g f \mathrm{fr}^{+}$ cells in 75 hpf OFTs. D) Quantification of EdU ${ }^{+}$SMCs between 48 and $72 \mathrm{hpf}$ in alk.5 $5^{+/+}$and alk.5\% OFTs. Mean \pm SD; $P$ values from $t$-tests. Figure adapted and reprinted with permission from (Boezio et al., 2020). License: CC BY 2.0.

Next, we took advantage of transmission electron microscopy (TEM) to investigate SMC ultrastructure. From these images, it was clearly visible a largely increased extracellular space surrounding SMCs in alk5 ${ }^{\%}$ OFTs (Figure 33A-B'), consistent with confocal images obtained with the $T g$ (pdgfrb:eGFP) line. I could also observe that the mutant extracellular space consisted of broad electron-negative spaces and disorganized clusters of molecules, which -due to the 
lack of labeling techniques- we were not able to identify. Moreover, I noticed that several SMCs in the external layer of the OFT displayed electron-dense cytoplasmic inclusions and doublemembraned vacuoles, reminiscent of phagosomes (Figure 33B').
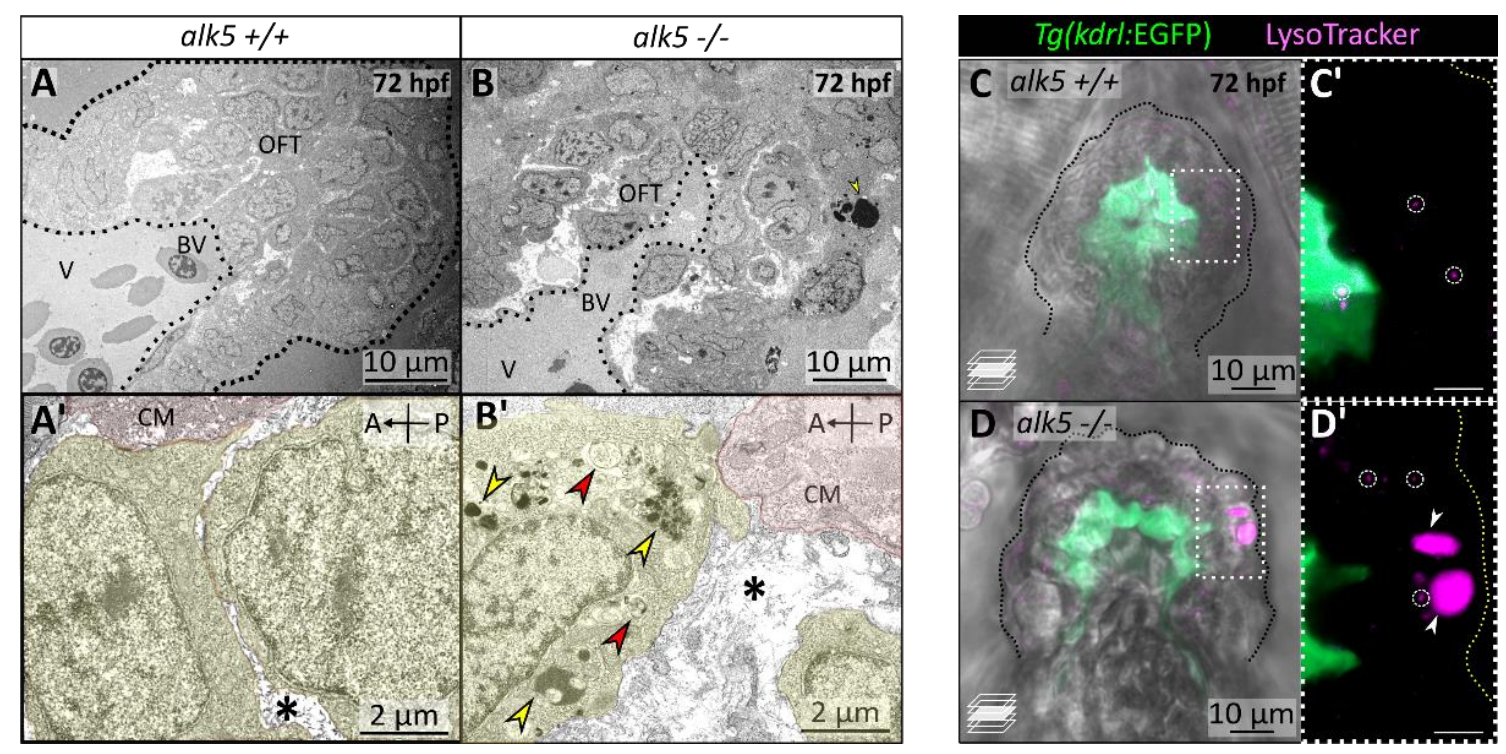

Figure 33: alk5 mutant SMCs contain acidic lysosomes and double-membraned structures.

A-B') TEM images of alk $5^{+/+}$(A) and alk $5 \%$ (B) OFTs, depicting SMCs in orange and CMs in red. Mutant SMCs exhibit double-membraned structures (red arrowheads) and electron-dense vacuoles (yellow arrowheads). C-D') Confocal pictures of wild-type and alk5 mutant OFTs treated with LysoTracker, labeling acidic lysosomes (magenta). alk $5^{+/+}$larvae exhibit only small-sized lysosomes (circles), while alk $5 \%$ SMCs display large acidic lysosomes (arrowheads). Figure adapted and reprinted with permission from (Boezio et al., 2020). License: CC BY 2.0.

To investigate the nature of these cytoplasmic inclusions, I used the Lysotracker, a fluorescent dye labeling acidic organelles in live cells. While in wild-type SMCs I could only detect small lysosomes (Figure 33C, C'), in alk $5^{-/}$larvae the Lysotracker labeled large vacuoles strongly resembling the TEM inclusions, and indicating the acidity of their content (Figure 33D, D'). Upon TUNEL staining, I could exclude that these inclusions were linked to apoptotic or necrotic cell death (data not shown). Nonetheless, TUNEL assay labels DNA fragmentation, a late stage of cell death. Therefore, the large lysosomes could be -together with the doublemembraned organelles- the first sign of subsequent cell death, possibly through autophagic processes. Currently, the exact nature of these inclusions remains to be determined.

Mature differentiated SMCs are commonly identified by markers like $\alpha$-smooth muscle actin (Acta2) or Transgelin (Tagln). With the aim of assessing the correct specification of SMCs in the zebrafish larvae, I immunostained $75 \mathrm{hpf}$ wild-type and alk 5 mutant hearts for these SMC markers (Figure 34A-D). Interestingly, very few wild-type SMCs exhibited faint staining for any of these markers, suggesting a still immature state of these cells (Figure 34A, C). 

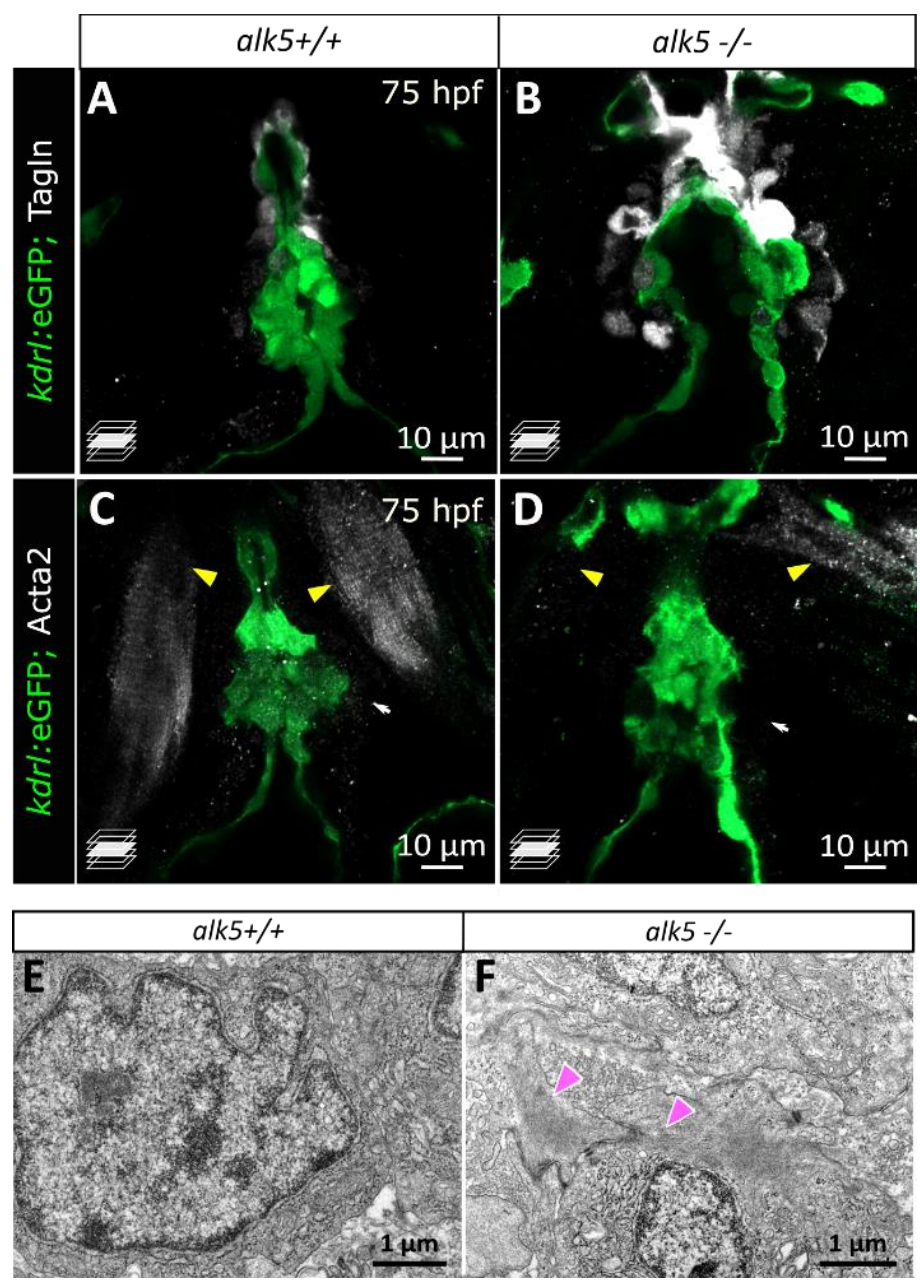

Figure 34: alk5/- larvae prematurely upregulate the expression of some mature SMC markers.

A-D) Confocal images of $75 \mathrm{hpf}$ alk.5\%/+ (A, C) and alk.5\% (B, D) showing the expression of Tagln (A, B) and Acta2 (C, D) proteins (white). alk5 mutants display a strong upregulation of Tagln in OFT SMCs. Yellow arrowheads point to Acta2 staining in the pharyngeal muscles. E, F) TEM images of $72 \mathrm{hpf}$ alk. $5^{+/+}$(E) and alk $5 \%$ (F) OFT SMCs. Arrowheads, actin bundles observed only in alk $5 \%$ SMCs.

In fact, in previous studies, an accumulation of $\operatorname{tag}^{+}{ }^{+}$cells around the dorsal aorta and the ventral artery has been shown only starting at 4 dpf (Whitesell et al., 2014; Ando et al., 2016; Stratman et al., 2017). Interestingly, in situ hybridization experiments for tagln show that its mRNA expression is already present at $75 \mathrm{hpf}$ (Santoro et al., 2009), suggesting this stage is crucial for SMCs maturation. Notably, alk.5 mutant SMCs exhibit a marked increase in the expression of Tagln (Figure 34B). As shown by TEM analyses, we could not observe any actin bundle in wild-type SMCs, but occasionally we found a few in alk.5 mutant SMCs, corroborating our immunostaining analyses (Figure 34E, F). Altogether, these data suggest that alk. $5^{-1-}$ SMCs at $75 \mathrm{hpf}$ have prematurely differentiated in more mature SMCs. Interestingly, this hypothesis could also partially explain their reduced proliferation rate, a typical sign of mature and quiescent cells. (Certain lines in this subsection have been quoted verbatim from Boezio et al., eLife 2020;9:e57603 for the scientific accuracy of the terms.) 


\subsubsection{Alk5 regulates the extracellular environment in the cardiac outflow tract}

To form a resistant and elastic OFT wall, SMCs need to be embedded in the ECM that fills the intercellular spaces. The ECM provides biomechanical support and signaling cues to the SMCs (Raines, 2000). Upon TEM analyses, I already identified wider extracellular gaps between mutant SMCs compared with wild-type (Figure 33A-B'). With the aim to understand the composition of the OFT ECM, I first analyzed the localization of its major component, Elastin2 (Eln2), by immunostaining (Miao et al., 2007). Elastin is responsible for the elastic recoil and extensibility in the large arteries, as well as in other non-vascular tissues. Among the two tropoelastin genes found in zebrafish, elna $(E \ln 1)$ and $e \ln b(E \ln 2), e \ln b$ is the predominantly expressed gene in the OFT, throughout the life of the fish (Miao et al., 2007). Indeed, I could observe a strong Eln2 immunostaining surrounding 77\% of wild-type SMCs, excluding the outermost layer (Figure 35A, C). In contrast, of the already fewer mutant SMCs, only 36\% were covered by continuous Eln2 immunostaining. Notably, elastin was not disposed in the characteristic bundles but rather localized in disrupted clusters (Figure 35B, B'), feature even more evident at 96 hpf (Figure 35D, E).
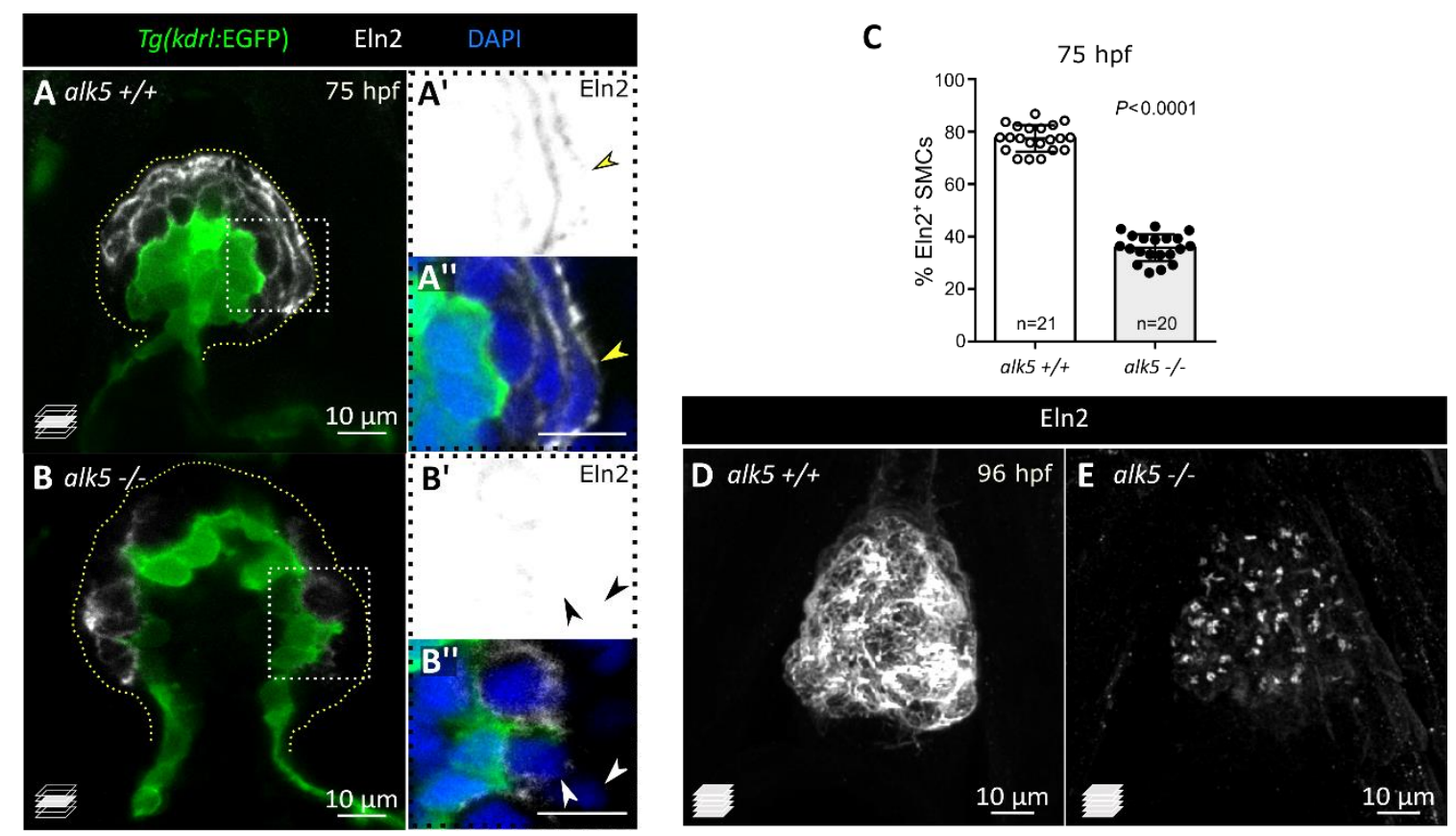

Figure 35: Alk5 regulates Eln2 deposition and organization around OFT SMCs.

A-B") Confocal images of $75 \mathrm{hpf}$ alk $5^{+/+}$(A) and alk $5 \%$ (B) OFTs immunostained for Eln2 (white). Eln2 staining surrounds most of the SMCs (yellow arrowheads) in alk $5^{+/+}$larvae, while it incompletely covers alk.51- SMCs (black/white arrowheads). Eln2 staining in A' and B' is shown in inverted colors. C) Quantification of the percentage of Eln $2^{+}$SMCs at 75 hpf. Mean \pm SD; $P$ value from $t$-test. D, E) Maximum intensity projections of $96 \mathrm{hpf}$ alk.5 $5^{+/+}$and alk.5\% OFTs showing Eln2 immunostaining in white. In alk $5 \%$, Eln2 is localized in disrupted clusters. Figure adapted and reprinted with permission from (Boezio et al., 2020). License: CC BY 2.0. 
The extracellular space surrounding SMCs is not only composed of Eln2, but its precise composition in zebrafish is yet unknown. I hypothesized, based on studies conducted on other model organisms, the presence of fibronectin (Fn1) and, thus I decided to assess its presence and localization in the OFT by immunostaining (Figure 36). In 96 hpf wild-type OFTs, Fn1 localized in the very thin extracellular space surrounding SMCs in continuous layers. Surprisingly, its immunostaining intensity was severely increased in alk.5 mutants OFT compared with wild-type (Figure 36A, B). It has been suggested in various contexts a prominent link between the SMC phenotype and the composition of the matrix (Raines, 2000; Kelleher et al., 2004; Shao et al., 2020). The increase in Fn1 expression has been associated with a more "synthetic" phenotype as opposed to a "contractile" one (L. Wang et al., 2012). Nonetheless, a SMC synthetic phenotype would be in contrast with the contractile phenotype suggested by the upregulation of mature SMC markers in alk. 5 mutants. It is important to remember that we still lack sufficient resolution to study the ultrastructural molecular organization of the components. It is well recognized that many ECM components are secreted in an inactive state and need further steps to form a resistant and compact network. Therefore, further studies will be needed to more precisely identify the exact composition and structure of the OFT ECM in physiological and pathological conditions.

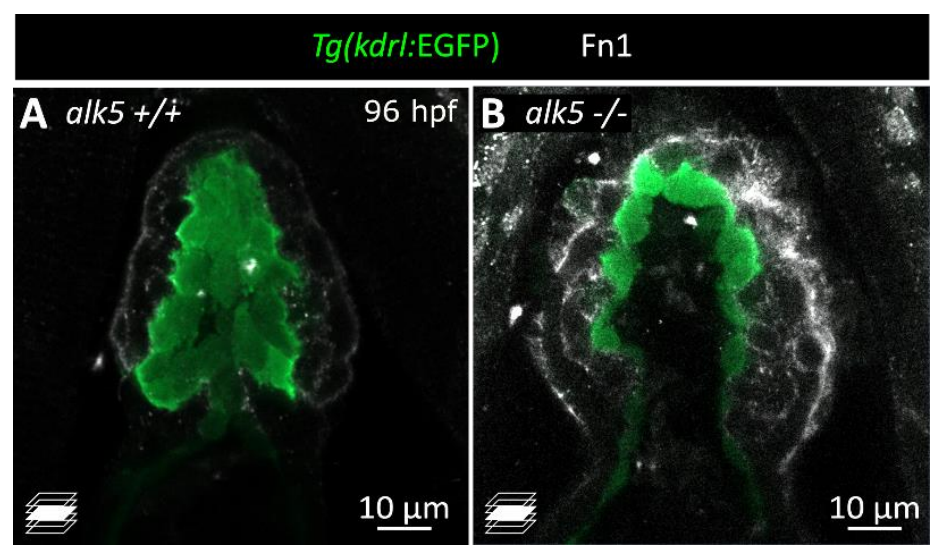

Figure 36: Fibronectin1 (Fn1) immunostaining is increased in alk5 $\%$-OFTs.

A, B) Confocal images of $96 \mathrm{hpf} \mathrm{Tg}(\mathrm{kdrl}: \mathrm{EGFP})$ alk $5^{+/+}$(A) and alk5\% (B) OFTs immunostained for Fn1 (white). Fn1 labels the intercellular space surrounding SMCs and its staining is increased in alk5\%- OFTs.

Altogether, these data suggest that Alk5 affects the ECM surrounding the developing OFT. Alk5 could regulate the deposition of the ECM, the relative proportion of its components, or their organization in the extracellular space. Of note, both ECs and SMCs contribute to the secretion of these molecules, strengthening the importance of identifying the tissue playing a primary role in the alk.5 mutant phenotype. (Certain lines in this subsection have been quoted verbatim from Boezio et al., eLife 2020;9:e57603 for the scientific accuracy of the terms.) 


\subsubsection{Alk5 is required in endothelial cells to promote OFT formation and function}

I previously identified the increased EC proliferation as the first detectable phenotype in alk. 5 mutant OFTs (Figure 30). Moreover, the OFT endothelium is also the first tissue in which the TGF- $\beta$ signaling pathway activates within the developing heart (Figure 16C, D). Therefore, when it came to identifying the primary tissue responsible for the alke $5^{-1}$ phenotype, I decided to focus on the endothelium. Given the global and constitutive nature of the alke 5 mutations, I could not distinguish between their cell-specific roles. Therefore, I designed a rescue construct overexpressing the wild-type alk $5 b$ cDNA under the control of an endothelial-specific promoter, fli1 a (Villefranc et al., 2007) (Figure 37A) To visualize the expression of the rescue transgene, I added to the construct a membrane-bound $\mathrm{mScarlet}$ sequence separated from alk $5 \mathrm{~b}$ by a P2A sequence. The P2A sequence ensures that all the cells expressing mScarlet will also express Alk5b, but the fluorophore will not interfere with the receptor's function. I chose to take advantage of alk. $5 b$ and not alk.5a for the rescue experiments since it proved to be the gene mostly responsible of the mutant phenotype. In fact, only one copy of wild-type alk $5 \mathrm{~b}$ in an alk $5 a^{-/}$background is sufficient to carry Alk5 function and sustain proper OFT morphology, as shown in Figure 21.

A
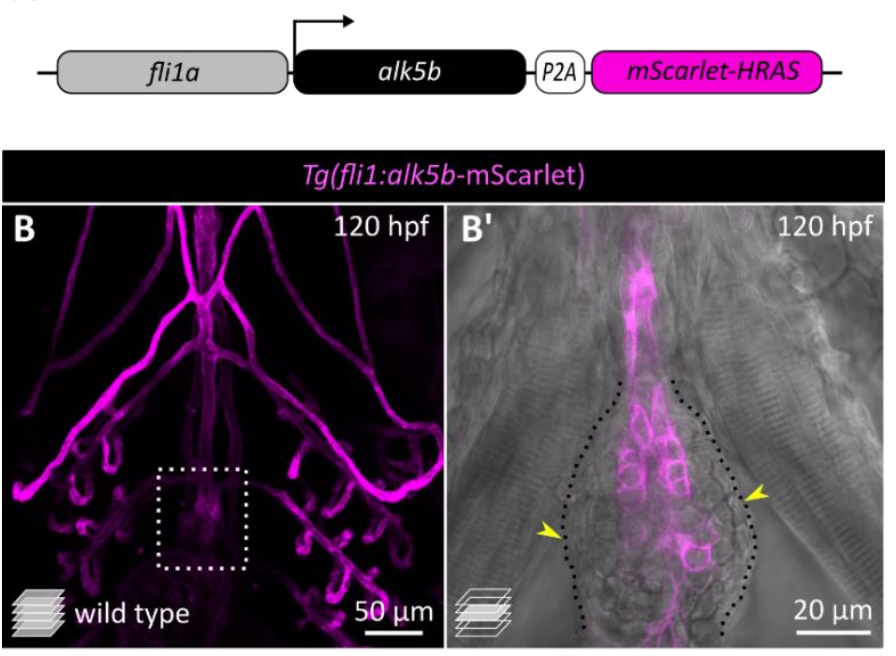

Figure 37: $T g$ (fli1a:alk5b-P2AmScarlet-hRAS) labels exclusively ECs and it does not affect cardiovascular development in wild-type larvae. A) Schematic of the flita:alk $5 b-P 2 A$ $m S$ carlet-hR $A S$ construct, overexpressing alk $5 b$ in ECs. B, B') The expression of $\mathrm{Tg}$ (flit a:alk5bP2A-mScarlet-hRAS) is limited to ECs and absent from SMCs (B', arrowheads). Figure adapted and reprinted with permission from (Boezio et al., 2020). License: CC BY 2.0.

At first, I injected the fli1a:alk5b-P2A-mScarlet-bRAS in wild-type embryos and raised them to adulthood. Both the mosaic F0 and the stable F1 generations survived to adulthood and were fertile. When I analyzed the expression pattern of the rescue construct in the larvae, I confirmed its exclusive expression in endothelial cells throughout the organism and its absence from OFT SMCs (Figure 37A, B). Notably, I could not detect any obvious defect in the cardiovascular patterning in the alk.5b overexpressing larvae (Figure 37A). This result suggests that the sole overexpression of the Alk5 receptor in a wild-type scenario is not sufficient to alter 
cardiovascular development and that additional regulatory steps are likely in place to prevent excessive activation of the signaling.

Subsequently, I injected the rescue construct in embryos derived from alk $5 a^{-1} ;$ alk. $5 b^{+/-}$intercross and observed alk.5 mutant larvae that expressed the rescue construct in a mosaic fashion. In the F0 injected embryos, very few (if any) fli1 a:alk $5 b-P 2 A-m S$ carlet- $b \mathrm{R} A S^{+}$cells localized to the OFT in both alk 5 mutants and controls, due to the very limited size of this endothelial compartment. Therefore, it was impossible to analyze a potential phenotypic rescue, so I decided to raise the stable F1 line carrying fli1 a:alk 5 b-P2A-mScarlet-bR AS in all endothelial cells.
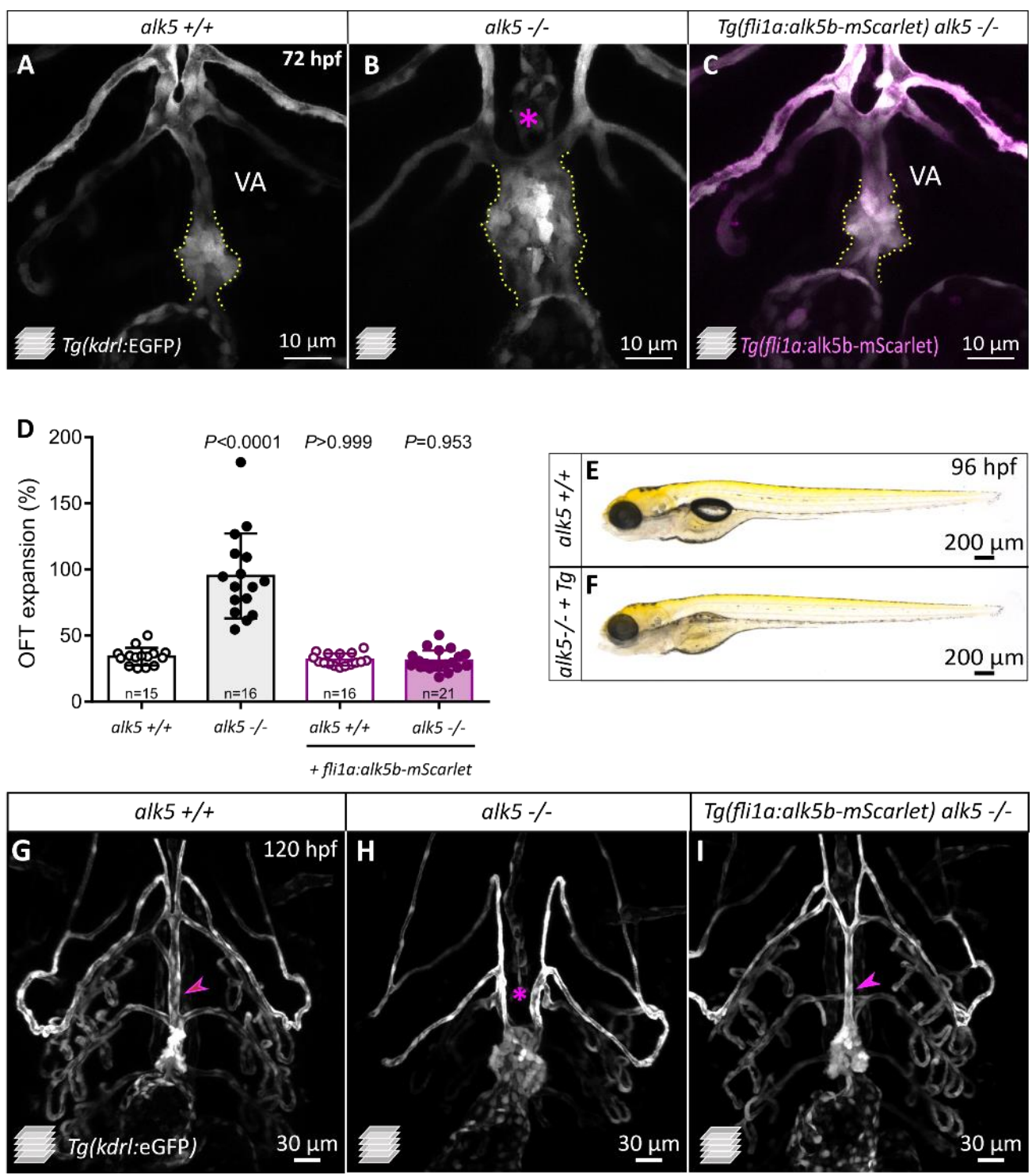

Figure 38: EC-specific overexpression of alk $5 \mathrm{~b}$ rescues OFT phenotypes in alk5 $/$ larvae.

A-C, G-I) Confocal images of 72 (A-C) and 120 (G-I) hpf OFTs in alk $5^{+/+}$, alk $5 \%$ larvae, and alk.5\% larvae carrying Tg(flita:alk5b-P2A-mScarlet-hRAS). D) Quantification of OFT expansion in $78 \mathrm{hpf}$ control larvae (grey) or larvae overexpressing the rescue transgene (magenta). Mean \pm SD; $P$ value from Kruskal-Wallis test, compared with the first column. E, F) Brightfield images of $96 \mathrm{hpf}$ alk $5^{+/+}$and Tgflit a:alk5b-P2A-mScarlethR AS) alk $5 \%$ larvae, showing no pericardial edema Figure adapted and reprinted with permission from (Boezio et al., 2020). License: CC BY 2.0. 
To my surprise, once I intercrossed Tg(fli1 a:alk $5 b-P 2 A$-mScarlet-bRAS) alk $5 a^{-1 /}$;alk $5 b^{+/-}$fish, I could not observe any morphological phenotype in the $96 \mathrm{hpf}$ alk.5 larva carrying the rescue transgene (Figure 38A-C, E, F). When analyzing OFT morphology and function at a higher resolution, I observed that $\mathrm{Tg}($ fli1a:alk $5 b-\mathrm{P} 2 A$-mScarlet-hRAS) alk.5 mutant OFTs were indistinguishable from wild types both in size and expansion $(P=0.953$ compared with wild types; Figure 38A-D). The VA patterning was also consistently restored, with $99 \%$ of the rescued mutant larvae exhibiting a complete VA formation, persisting until later larval stages (Figure 38C, G-I).

With the aim to understand which cellular phenotypes were effectively rescued by the alk $5 \mathrm{~b}$ EC-specific overexpression, I first analyzed EC proliferation by EdU assay (Figure 39A). Between 48 and $72 \mathrm{hpf}$, the percentage of EdU ${ }^{+}$ECs was consistently reduced in Tgfli1 a:alk:5bP2A-mS carlet-hR AS) alk 5 mutants compared with alk 5 mutants not carrying the transgene, and identical to wild types ( $P>0.999$ compared with wild types; Figure 39A). Moreover, the percentage of $\mathrm{EdU}^{+}$SMCs at the same stage was increased compared with alk5 mutants not carrying the rescue construct, reaching levels similar to controls (Figure 39B). Therefore, the overexpression of alk.5b exclusively in ECs is able not only to rescue EC proliferation but also to restore SMC proliferation and number around the OFT. Moreover, overexpressing alk. $5 b$ in alk 5 mutant larvae was sufficient to restore Eln2 coverage around the OFT. $71.9 \%$ of SMCs were surrounded by Eln2 coverage in rescued alk 5 mutants, compared with $77.6 \%$ in wild-type animals and approximately 36\% in alke 5 mutants (Figure 39C-F).
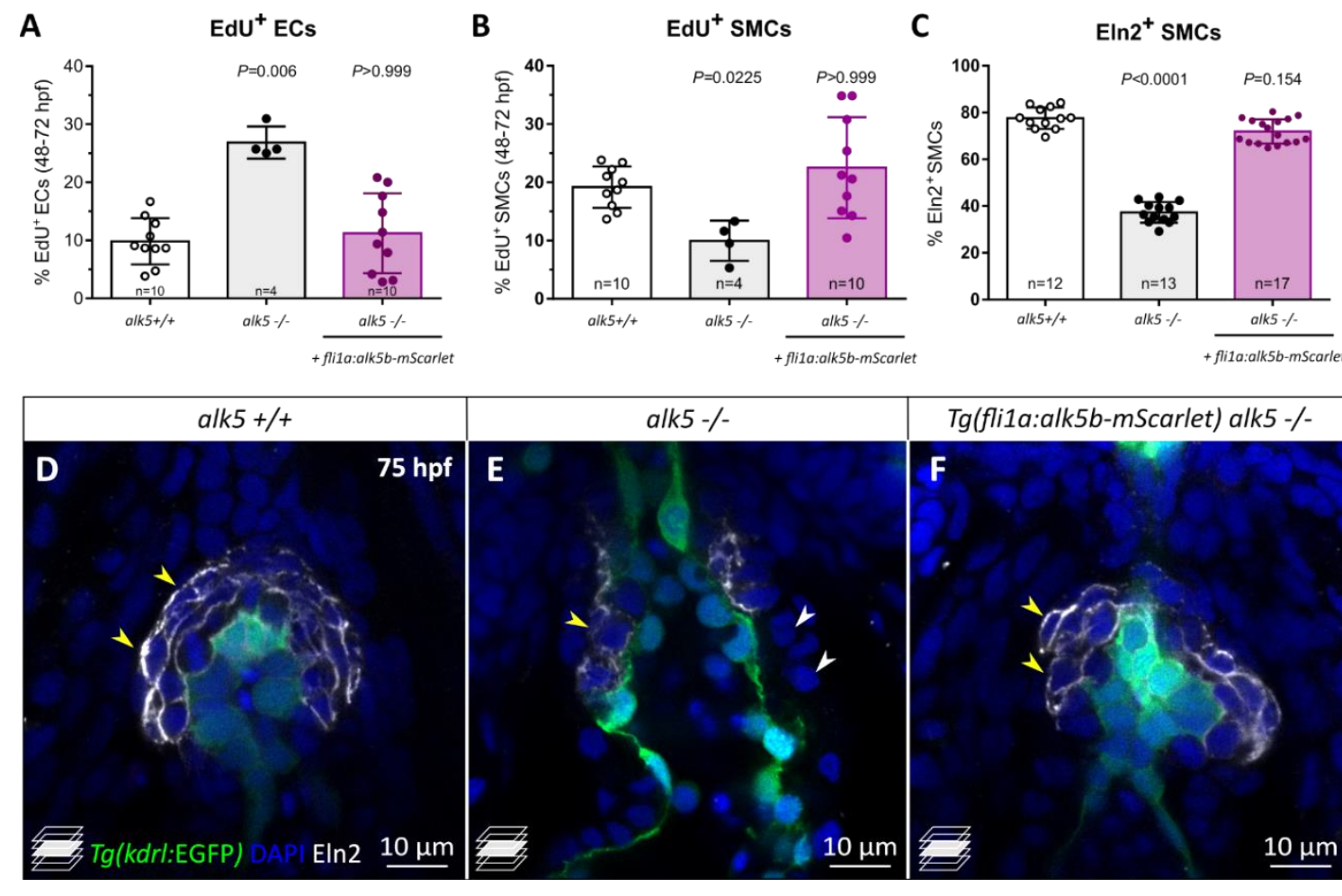

Tg(fli1a:alk5b-mScarlet) alk5 -/-

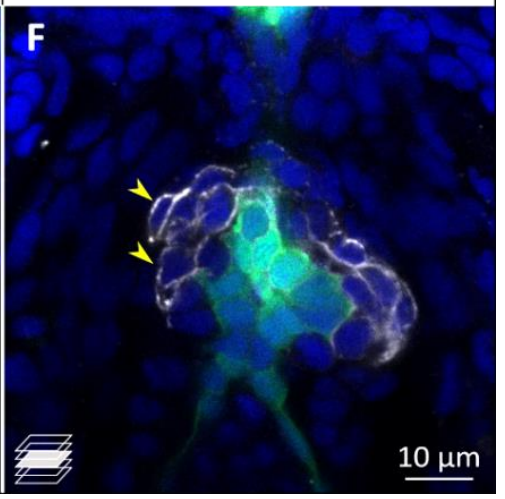

Figure 39: EC-specific alk5 overexpression restores EC and SMC proliferation, and Eln2 coverage. 
Figure 39: EC-specific alk5 overexpression restores EC and SMC proliferation, and Eln2 coverage. (previous page)

A-C) Quantification of EdU ${ }^{+}$ECs (A), EdU ${ }^{+}$SMCs (B) and Eln2 covered SMCs (C) in 72 (A, B) or 75 (C) hpf control larvae (grey) or larvae overexpressing the rescue transgene (magenta). Mean \pm SD; $P$ value from One-way ANOVA or Kruskal-Wallis tests. D-F) Confocal images of $75 \mathrm{hpf} \mathrm{larvae,} \mathrm{showing} \mathrm{the} \mathrm{Eln2} \mathrm{(white)}$ coverage of OFT SMCs (yellow arrowheads). White arrowheads in E point to SMC not covered by Eln2. Figure adapted and reprinted with permission from (Boezio et al., 2020). License: CC BY 2.0.

The restoration of the vascular patterning and cardiac function allowed alk.5 mutants to survive longer, due to a normal blood flow in the organism. Approximately $10 \%$ of Tgffli1 a:alk.5b-P2A$m S$ carlet-hR AS) alk5 mutants ( $\mathrm{n}=108)$ even inflated their swim-bladder (Figure 40). This result raises new questions on the swim-bladder phenotype and its dependency on blood flow or SMC behavior, which will be an interesting starting point for future studies. Interestingly, even the inflation of the swim bladder did not allow the larvae to live past $11 \mathrm{dpf}$, likely due to defects in other organs or tissues, as suggested by a malformation of the head (Figure 40B). (Certain lines in this subsection have been quoted verbatim from Boezio et al., eLife 2020;9:e57603 for the scientific accuracy of the terms.)
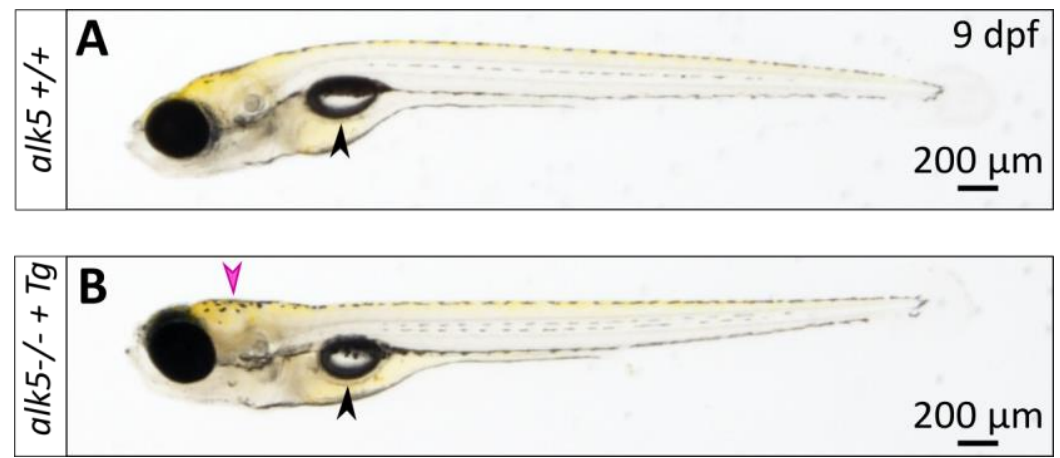

Figure 40: Endothelial alk5b overexpression rescues swim bladder inflation in $10 \%$ of alk5/- larvae. A-B) Brightfield images of $9 \mathrm{dpf}$ alk $55^{+/+}$(A) and Tgffli a:alk 5 b-P2A-mScarlet-hRAS) alk $5 \%$ (B) larvae. ECspecific overexpression of alk.5b rescues the inflation of the swim bladder (black arrowhead). Magenta arrowhead, malformation of the head. Figure adapted and reprinted with permission from (Boezio et al., 2020). License: CC BY 2.0.

\subsubsection{Alk5 signaling regulates ECM gene expression to orchestrate OFT development}

Based on the previous results, Alk5 seems required in the endothelium to orchestrate OFT development. Interestingly, recovery of Alk5 expression in ECs is sufficient to restore ECM and SMC morphology, organization, and function. These data suggest that Alk5 triggers a crosstalk between ECs and SMCs, possibly via the secretion of signaling molecules.

Next, I aimed to investigate the key molecular effectors signaling between these tissues and how they are affected when Alk5 function is impaired. To gain an unbiased insight, I performed transcriptomic analyses and took advantage of the Alk5 inhibitor. After treating the embryos 
with E-616452 with the previously described settings, I manually extracted larval hearts of control and treated larvae at $56 \mathrm{hpf}$ (Figure 41A). I chose $56 \mathrm{hpf}$ as a time window to identify the earlier molecular signaling taking place between ECs and SMCs before the excessive expansion of the OFT would trigger additional secondary effects. While dissecting the hearts, I also extracted the region of the OFT together with a limited portion of the surrounding mesoderm.

A

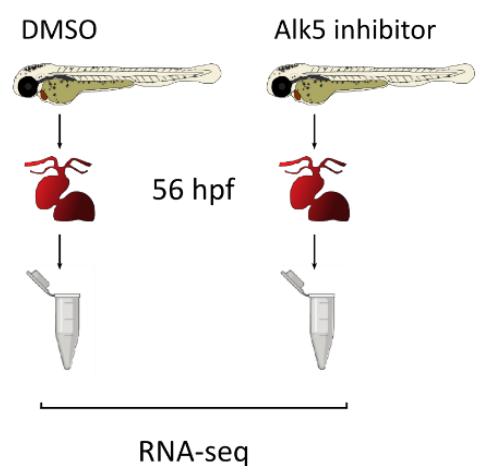

B

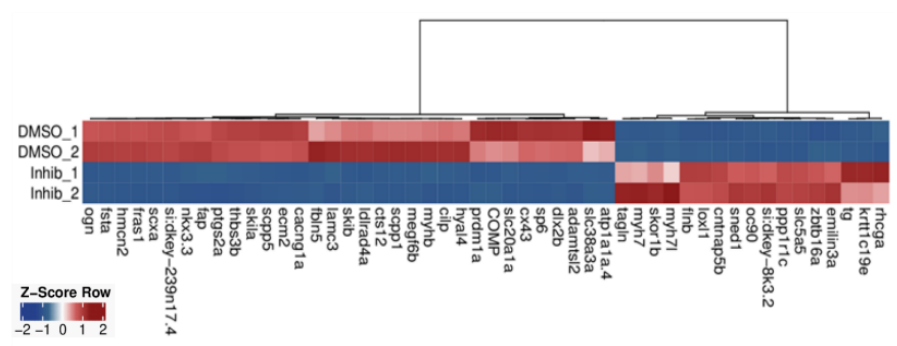

Figure 41: Setting of the RNA-seq on embryonic hearts following Alk5 inhibition.

A) Schematic depicting RNA-seq setting on extracted $56 \mathrm{hpf}$ hearts, following Alk5 inhibition. B) Heat map of the top 50 differentially expressed genes (DEGs) between Alk5 inhibitor-treated and DMSO-treated 56 hpf hearts. Figure adapted and reprinted with permission from (Boezio et al., 2020). License: CC BY 2.0.

Compared to DMSO-treated larval hearts, Alk5 inhibitor-treated hearts exhibited 955 differentially expressed genes (DEGs), out of which 480 were downregulated (Figure 41B). Notably, upon Gene Ontology analyses (Ashburner et al., 2000; Gene Ontology, 2021), the ECM-related terms (extracellular space, extracellular matrix, ECM organization) were consistently found among the most downregulated ones (Figure 42). The ECM has been proved to be a crucial platform of communication between different cell types, acting both at a structural level, as well as a reservoir of signaling molecules.

A

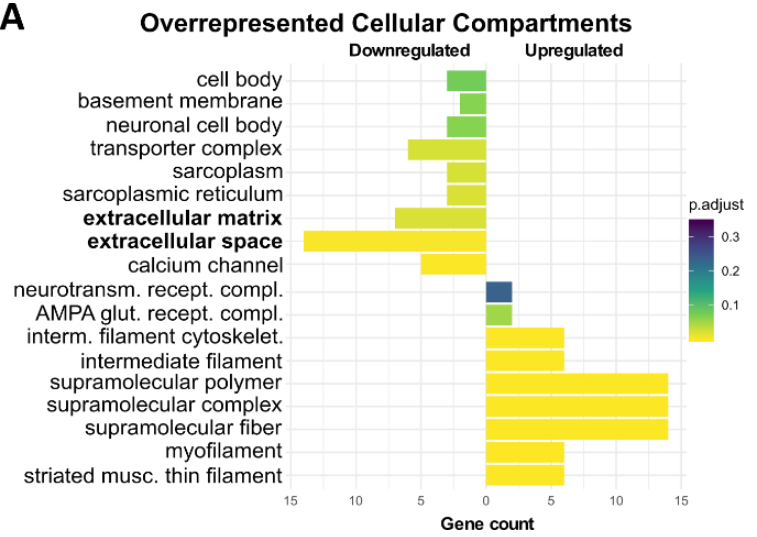

B Overrepresented Biological Processes (downregulated)

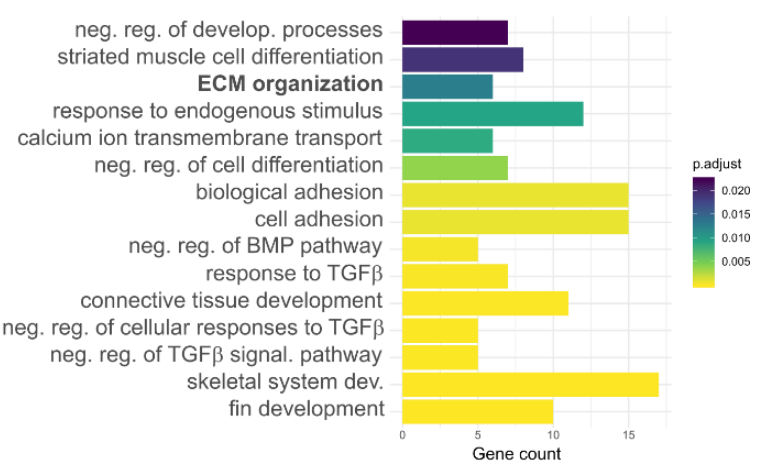

Figure 42: Alk5 regulates the expression of several ECM component genes. 
Figure 42: Alk5 regulates the expression of several ECM component genes. (previous page)

A-B) Gene ontology bar plots, showing overrepresented GO terms (A, cellular compartments; B, biological processes) among downregulated and upregulated genes in DMSO- and Alk5 inhibitor-treated hearts. Figure adapted and reprinted with permission from (Boezio et al., 2020). License: CC BY 2.0.

I then aimed at selecting genes encoding for secreted proteins, which could be released in the extracellular space and act as mediators of the signaling. Thus, to narrow down the list of DEGs, I first compared them with secreted component genes present in the zebrafish matrisome (Nauroy et al., 2018) and identified 82 genes. Then, to further narrow down the list, I compared these 82 genes with the ones specifically expressed in the adult bulbus arteriosus (BA; another name used to refer to the zebrafish OFT) (A. R. Singh et al., 2016) (Figure 43A).

A

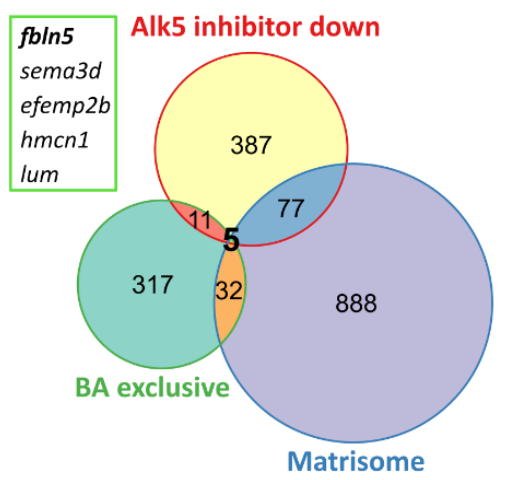

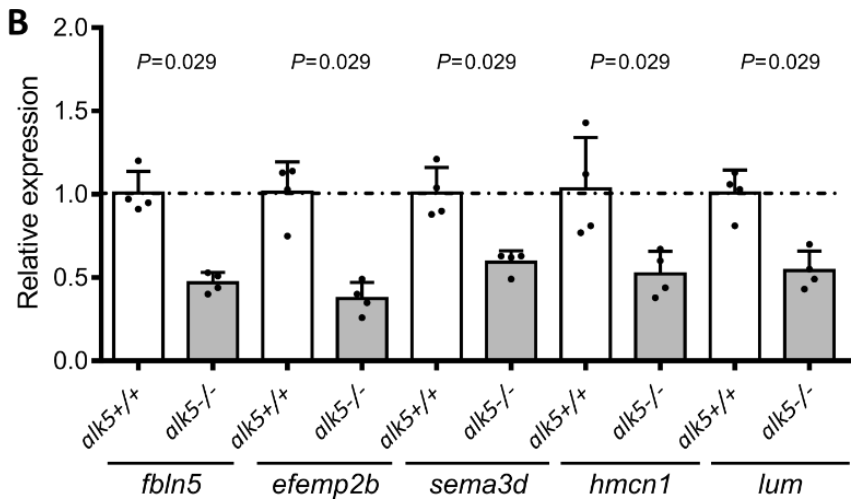

Figure 43: Alk5 regulates the expression of secreted OFT-specific ECM genes.

A) Venn diagram comparing genes downregulated in Alk5 inhibitor-treated hearts, belonging to the zebrafish Matrisome (Nauroy et al., 2018) and specific of the zebrafish bulbus arteriosus (BA) (A. Singh et al., 2019). B) Relative mRNA expression of the 5 genes selected in A in $72 \mathrm{hpf}$ alk $5^{+/+}$and alk5 $5 \%$ hearts. Mean \pm SD; $P$ value from Mann-Whitney tests. Figure adapted and reprinted with permission from (Boezio et al., 2020). License: CC BY 2.0.

Comparing the three datasets allowed me to identify transcripts that were dysregulated in absence of Alk5 function, encoding for secreted proteins, and specifically expressed in the OFT. Such cross-referencing revealed 5 genes, accounting also for the specificity of the OFT defects: fbln 5 , efemp $2 b$ (fbln4), sema3d, hmon1, lum. After validating their downregulation in $72 \mathrm{hpf}$ alk. 5 mutant extracted hearts by qPCR (Figure 43B), I decided to focus on the most downregulated gene, fbln5. (Certain lines in this subsection have been quoted verbatim from Boezio et al., eLife 2020;9:e57603 for the scientific accuracy of the terms.)

\subsubsection{The OFT-enriched ECM component gene $f b l n 5$ acts downstream of Alk5 to regulate EC proliferation and ECM organization}

Mammal Fbln 5 encodes an actin-binding extracellular protein, expressed mainly in the aorta and outflow tract during embryogenesis, both in ECs and fibroblasts/SMCs (Yanagisawa et al., 2009; X. Liu et al., 2019). Fbln5 has an arginine-glycine-aspartic acid (RGD) domain in the N- 
terminal region that mediates the binding to integrins. Furthermore, it has been shown to be a target of Smad3 and TGF- $\beta$ in vitro (Schiemann et al., 2002). Its functions reported in literature also proved to be extremely interesting in light of the alk.5 mutant phenotype. Fbln 5 mediates EC attachment to the matrix, thereby inhibiting their proliferation (Albig and Schiemann, 2004; Preis et al., 2006; Sullivan et al., 2007; Williamson et al., 2007), while in fibroblasts it exerts the opposite effect promoting DNA synthesis (Schiemann et al., 2002). Moreover, as an ECM component, it proved to be crucial for in vivo elastogenesis. Fbln 5 is necessary for the formation of the elastic fibers from tropoelastin monomers (Nakamura et al., 2002; Yanagisawa et al., 2009; Chapman et al., 2010).

Therefore, I first decided to investigate its expression pattern in zebrafish and found that $f b \ln 5$ serves as an extremely specific OFT marker at $72 \mathrm{hpf}$, like no other known gene (Figure 44A, B). Its mRNA expression was already visible in the region of the OFT at $36 \mathrm{hpf}$, although the staining was very faint (Figure 44C, C'). However, the whole mount colorimetric in situ hybridization is not informative in terms of cellular localization and lacks sensitivity for low expressed genes. It was crucial for our study to understand which cells expressed $f b l n 5$, especially at early time points. Therefore, I performed fluorescent in situ hybridization at $36 \mathrm{hpf}$ and observed $f b \ln 5$ expression in the forming OFT and aortic arches (Figure 44D). Notably, fbln 5 expression was localized at the level of both mesodermal cells and ECs in the OFT, confirming its expression at least partially from the endothelium (Figure 44D').
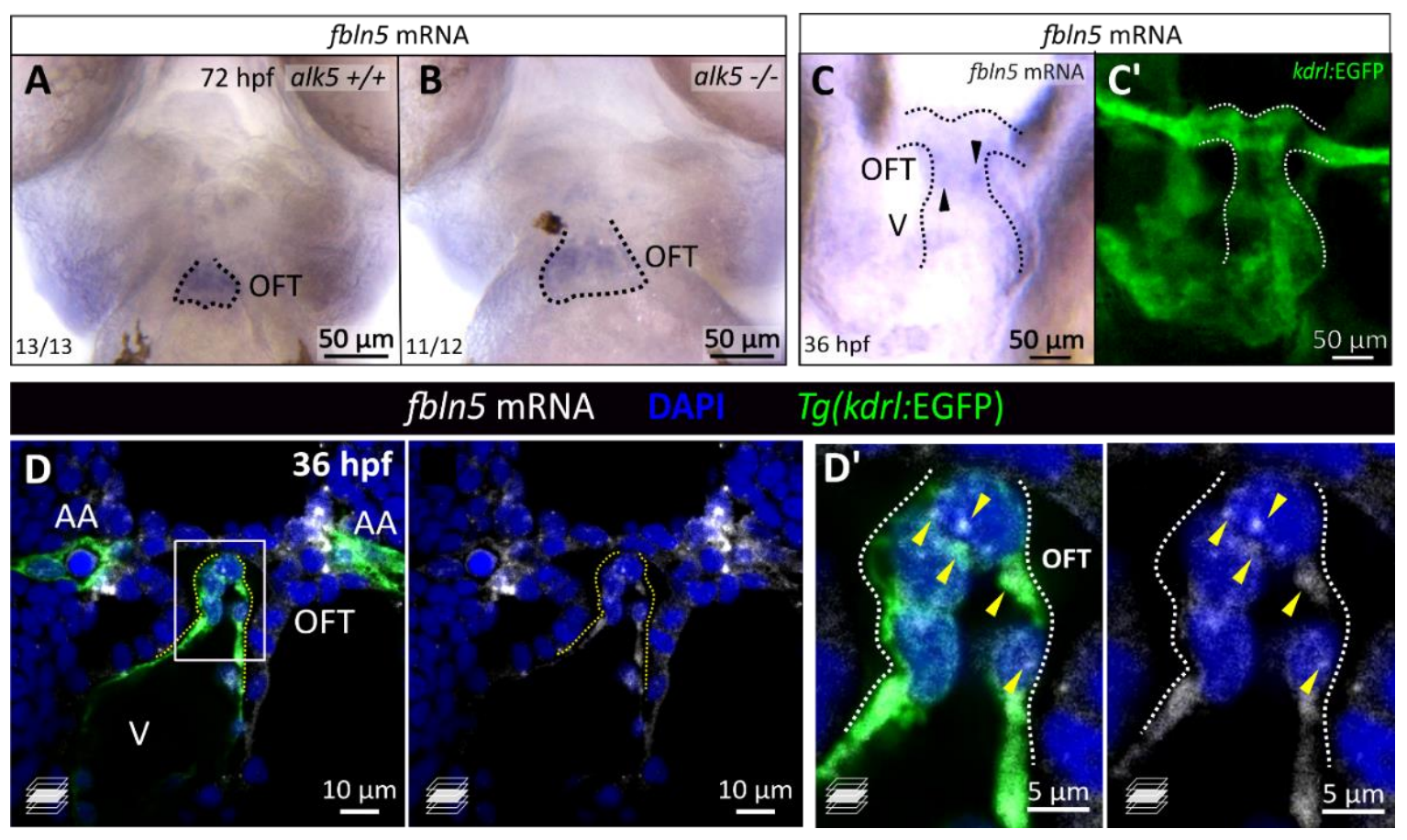

Figure 44: $f b \ln 5$ is specifically expressed in the OFT in both ECs and surrounding cells.

A-C') Whole-mount ISH images showing the expression of $f b \ln 5 \mathrm{mRNA}$ in the 72 (A) and 36 (C) hpf OFTs. Arrowheads, staining in the OFT; dashed lines, OFT. $f b \ln 5$ is expressed also in alk $5 \%$ OFT (B), although at lower levels. (Figure legend continues on the next page) 
D, D') Fluorescent ISH images showing the expression of $f b \ln 5 \mathrm{mRNA}$ (white) in $36 \mathrm{hpf}$ OFT ECs and aortic arches (AA). Boxed area shown in D'; arrowheads point to punctae of $f b \ln 5$ mRNA in OFT ECs. Figure adapted and reprinted with permission from (Boezio et al., 2020). License: CC BY 2.0.

Once I identified the cell types expressing $f b \ln 5$, I aimed at understanding if this gene could be a target of Alk5 in zebrafish. Therefore, I analyzed fbln 5 expression in alk.5 mutant hearts compared with Tg(fli1 a:alk.5b-P2A-mScarlet-bRAS) alk.5 mutant hearts. The expression of fbln 5 was partially increased in the mutants carrying the rescue transgene, although at a low level (Figure 45). Notably, the expression of $f b \ln 5$ was higher in the pools of hearts with a higher expression of alk.5b. This result indicates that the expression of $f b \ln 5$ correlates with the level of alk.5, suggesting that $f b \ln 5$ is a potential target gene of the TGF- $\beta$ pathway.

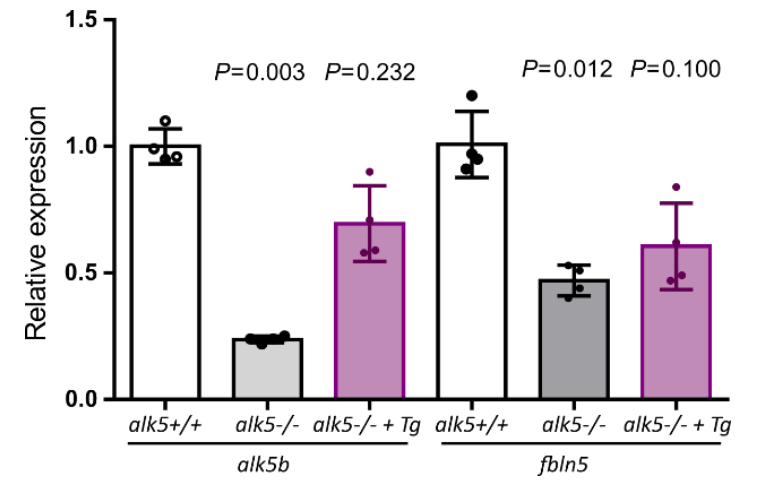

Figure 45: The overexpression of alk5b in ECs partially rescues $f b \ln 5$ levels in alk 5 mutants Relative alk $5 b$ and fbln 5 mRNA expression in alk $5^{+/+}$, alk $5 \%$, and fli1 a:alk $5 b-P 2 A-m S$ carlet-bR AS alk. $5 \%($ alk. $5 \%+T g)$ hearts at $72 \mathrm{hpf}$. Mean \pm SD; $P$ value from Kruskal-Wallis tests comparing all the columns with alk $5^{+/+}$. Figure adapted and reprinted with permission from (Boezio et al., 2020). License: CC BY 2.0.

Subsequently, I aimed at understanding the contribution of Fbln 5 to the alk 5 mutant OFT phenotype. As a preliminary approach, I injected $150 \mathrm{pg} f b \ln 5 \mathrm{mRNA}$ (and GFP mRNA as a control) in embryos deriving from alk $5 \mathrm{a}^{-/}$; alk $5 \mathrm{~b}^{+/-}$intercrosses. I first assessed the OFT largest area at $78 \mathrm{hpf}$ in control and $f b \ln 5$-injected larvae. The injection of $f b \ln 5 \mathrm{mRNA}$ significantly reduced the size of the OFT in $71.4 \%$ alk 5 mutants $(n=21 ; P<0.0001)$, while it did not have any effect in wild-type siblings (Figure 46A).

Since the overexpression of fbln 5 with mRNA injections does not allow one to distinguish between its tissue-specific contributions, I decided to assess EC proliferation at early developmental stages (24-36 hpf), before SMC coverage. Notably, the injection of $f b \ln 5$ mRNA did not affect EC proliferation in wild-type OFTs, but it decreases the number of EdU ${ }^{+} \mathrm{ECs}$ in alk5 mutants (Figure 46B). These data suggest that Fbln5 is expressed in the early endothelium of the OFT and restricts EC proliferation in alk 5 mutant embryos. The lack of effects on wild-type ECs suggests the existence of a maximum Fbln5 (mRNA or protein) threshold, after which it does not increase its functionality. 
A

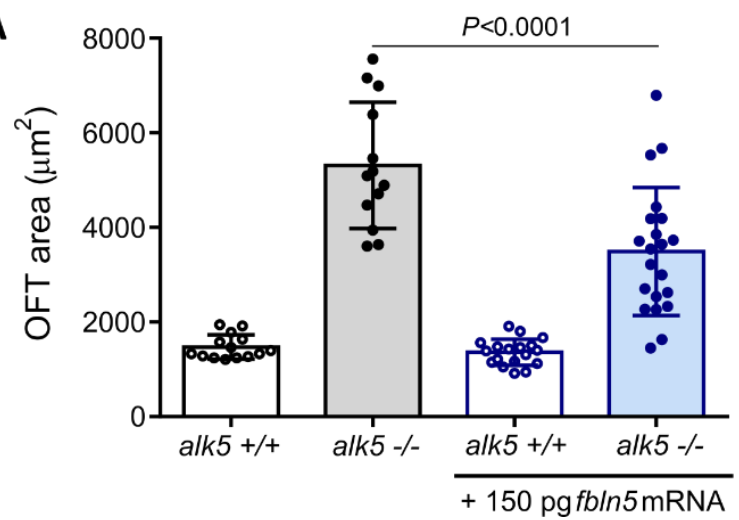

B

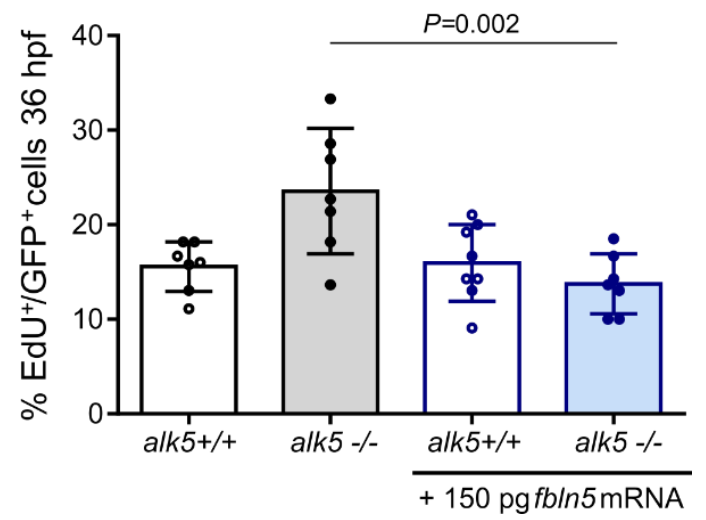

Figure 46: $f b \ln 5$ mRNA injection partially rescues OFT defects in alk5/- animals.

A) Quantification of OFT expansion in $72 \mathrm{hpf}$ alk $5^{+/+}$and alk.5\% larvae, following $f b l n 5 \mathrm{mRNA}$ or control injection. B) Quantification of EdU ${ }^{+}$ECs $\%$ in $36 \mathrm{hpf}$ alk $5^{+/+}$and alk $5^{-/}$larvae, following $f b \ln 5 \mathrm{mRNA}$ or control injection. Mean \pm SD; $P$ value from Mann-Whitney tests between the selected columns. Figure adapted and reprinted with permission from (Boezio et al., 2020). License: CC BY 2.0.

To address more precisely the cellular contribution of Fbln5, I generated a construct to overexpress $f b \ln 5$ specifically in endothelial cells (Figure 47A). I cloned the cDNA of $f b \ln 5$ downstream of the fli1 a bidirectional promoter, which also controls on the opposite strand the expression of GFP. With this tool, cells expressing $f b \ln 5$ will also be fluorescent with GFP, without hindering Fbln5 function, possible risk with a tagged version of Fbln5. With this approach, I observed a reduction in OFT expansion at $78 \mathrm{hpf}$ (Figure 48A), as well as the formation of the VA, in several $\mathrm{Tg}$ (fli1 a:fbln5,EGFP) alk $5^{-1}$ larvae (Figure 47B-D).

A
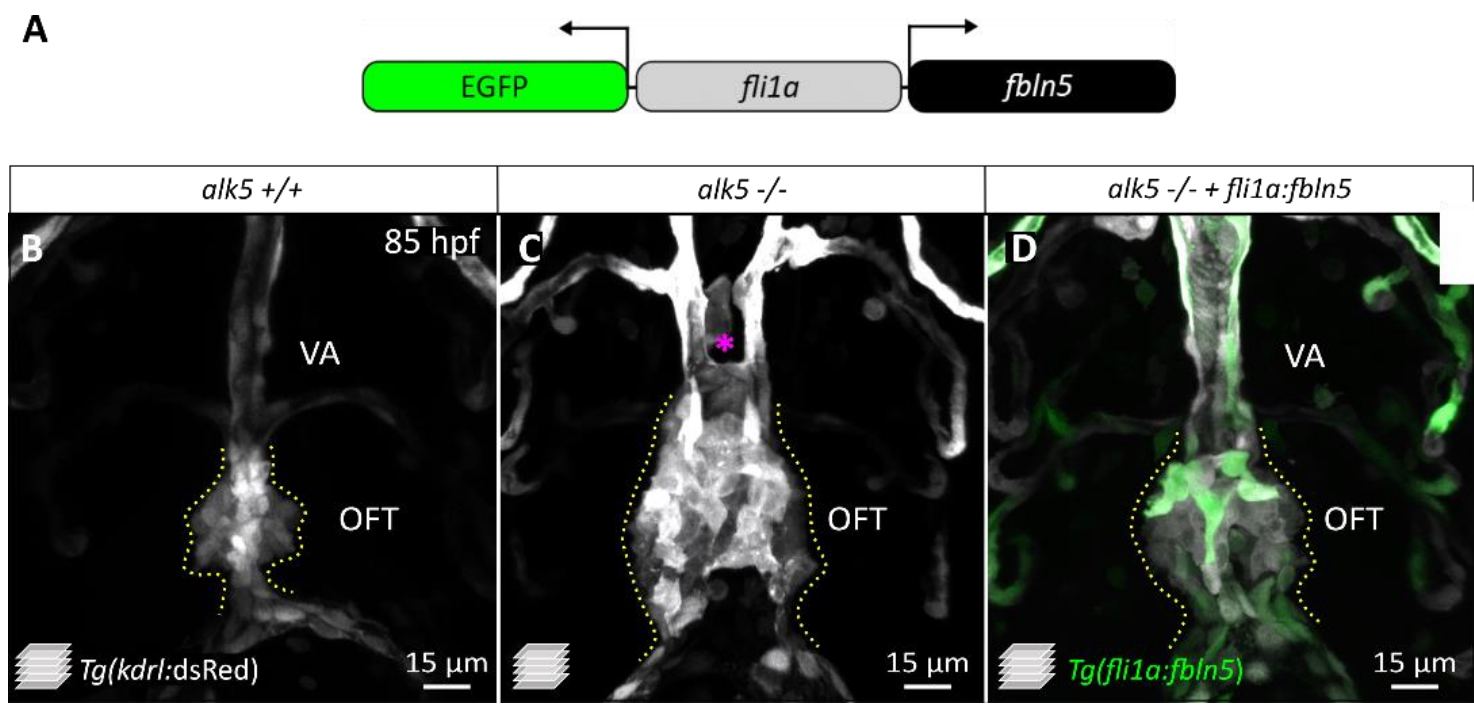

Figure 47: EC-specific $f b l n 5$ overexpression partially rescues OFT morphology in alk5/- animals. A) Schematic representing the Tg(flit a:fbln5,EGFP) construct. B-D) Confocal images of $85 \mathrm{hpf}$ alk:5 $5^{+/+}$, alk.51- (B, C), and alk $5 \%$ OFTs overexpressing $f b \ln 5$ (green) in ECs (D). Figure adapted and reprinted with permission from (Boezio et al., 2020). License: CC BY 2.0. 
Moreover, when I quantified the number of SMCs covered by Eln2 immunostaining, I observed a significant increase (58.5\%) in Tg(fli1 a:fbln5,EGFP) alk.5 animals, compared with alk.5 mutants not rescued (31.1\%) (Figure 48B-E). Similar coverage of Eln2 could be achieved also with the injection of $f b \ln 5$ (55.6\%; data not shown), suggesting that many functions of Fbln 5 can be carried out -when needed- by the EC-secreted protein only. (Certain lines in this subsection have been quoted verbatim from Boezio et al., eLife 2020;9:e57603 for the scientific accuracy of the terms.)
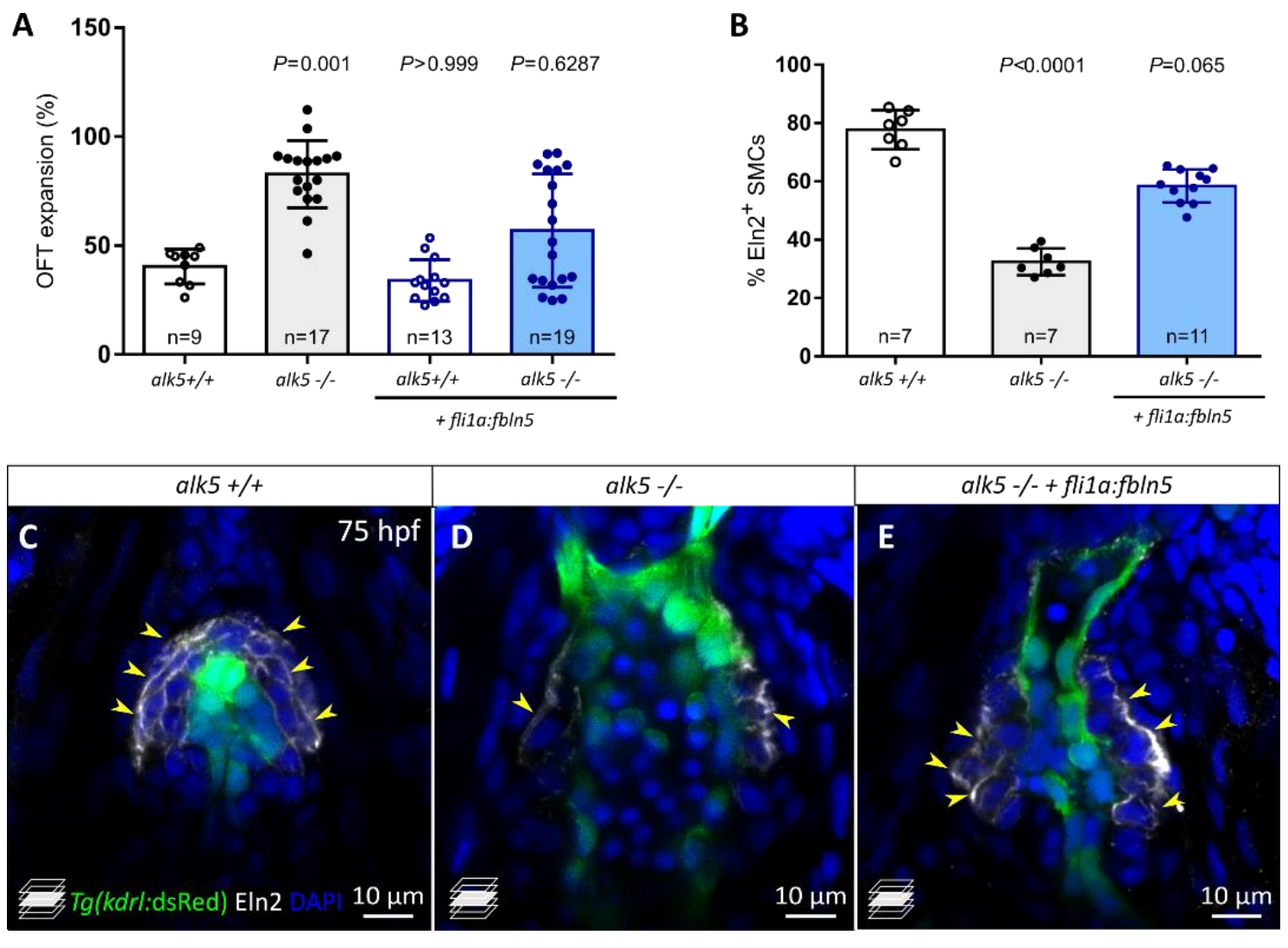

Figure 48: EC-specific $f b \ln 5$ overexpression partially rescues OFT function and ECM organization in alk5\%- animals.

A) Quantification of OFT expansion at $78 \mathrm{hpf}$, comparing larvae carrying Tg(fli1 a:fbln5,EGFP) with controls.

B) Quantification of Eln2 coverage in $78 \mathrm{hpf}$ OFTs, comparing control larvae and animals carrying Tg(fli1 a:fbln5,EGFP). Mean \pm SD; $P$ values from Kruskal-Wallis tests compared with the first column. C-E) Confocal images of $75 \mathrm{hpf}$ OFTs, showing a rescued Eln2 staining in alk5\% larvae carrying Tg(fli1 a:fbln5,EGFP). Figure adapted and reprinted with permission from (Boezio et al., 2020). License: CC BY 2.0.

\subsubsection{Proposed model}

In this study, I propose that Alk5/TGF- $\beta$ signaling controls the EC/SMC crosstalk during cardiac outflow tract development in zebrafish (Figure 49). In particular, I observed that:

1) Smad 3 / TGF- $\beta$ signaling downstream of Alk 5 is initially activated in the OFT endothelium 
2) Mutation of alk.5 causes a very specific dilation of the OFT, started by an increased proliferation of ECs and their impaired displacement. Subsequently, Alk5 signaling orchestrates ECM deposition/organization, and SMC proliferation.

4) Endothelial Alk5 is sufficient to rescue EC, as well as ECM and SMC defects in the alk.5 mutant outflow tract, indicating its prominent role in endothelial cells.

5) ECM components are particularly affected by the loss of Alk5. In particular, fbln 5 specific OFT expression is downregulated and its endothelial overexpression partially rescues the OFT phenotype.

Overall, I propose that Alk5 controls the EC-secreted ECM environment, which in turn could affect both EC and SMC proliferation and behavior in regulating OFT formation.

- Increased EC proliferation

- Alteration of ECM deposition

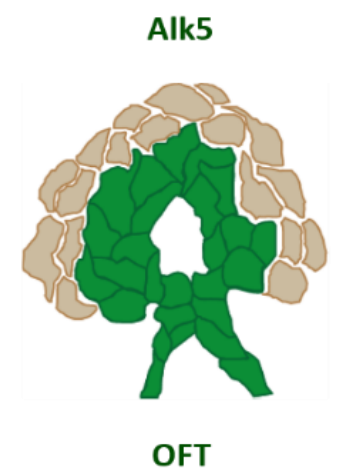

OFT

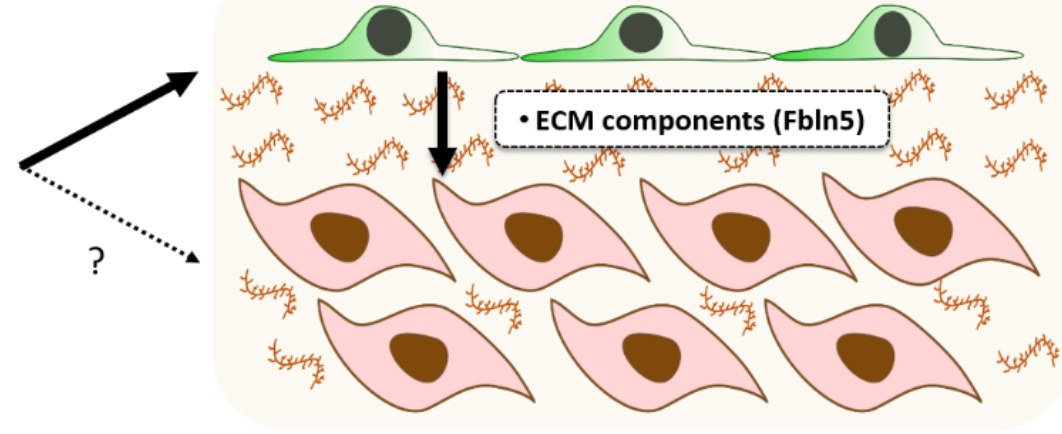

- Alteration of ECM deposition

- Reduced SMC proliferation
ECs

ECM

SMC

Figure 49: Proposed model (see text). 


\subsection{The epicardium-myocardium crosstalk is essential for cardiomyocyte growth during zebrafish cardiac development}

\subsubsection{Generation of independent models of impaired epicardial coverage in zebrafish larvae}

The epicardium is the last layer of cells added to the heart during cardiac development. After epicardial cell delamination from the proepicardium, these cells start adhering to the myocardial wall (Peralta et al., 2013, Simoes and Riley, 2018). In zebrafish, this event takes place around 52-56 hpf and, over few days, epicardial cells entirely envelop the ventricle (Peralta et al., 2014) (Figure 50A). At $72-76 \mathrm{hpf}$, approximately 50\% of the ventricle and the OFT are covered by epicardial cells, while at $100 \mathrm{hpf}$, the ventricle shows an almost complete epicardial coverage (Figure 50B). The epicardial coverage of the atrium starts in late larval stages and is complete by juvenile stages. Eventually, adult zebrafish hearts are completely covered by epicardial cells (Figure 50C).

A
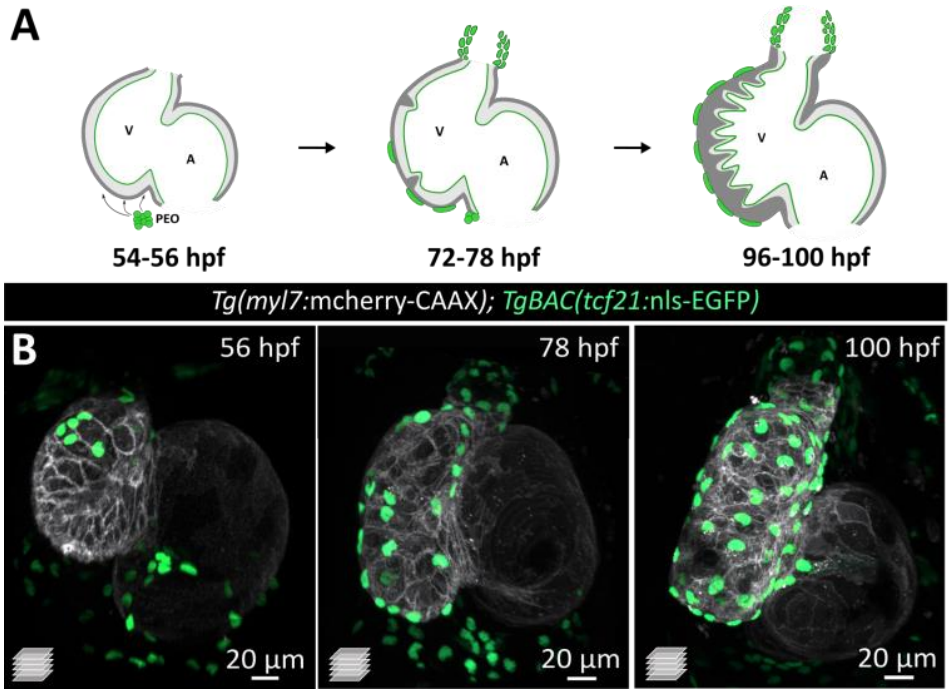

C

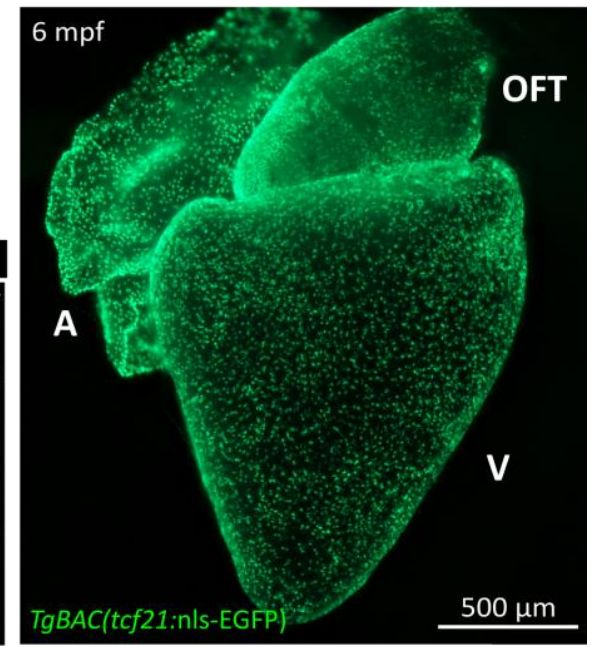

Figure 50: Epicardial coverage of the zebrafish embryonic, larval and adult heart

A) Schematic representing the epicardial coverage of the zebrafish embryonic and larval hearts. A, atrium; $\mathrm{V}$, ventricle; PEO, proepicardial organ. B-C) Confocal images of TgBAC(ttf21:nls-EGFP);(myl7:mCherry$C A A X)$ embryonic, larval and adult (C) hearts, exhibiting different extent of epicardial coverage (green, epicardial nuclei). $\operatorname{tc} 21^{+}$cells not attached to the ventricle in $\mathrm{B}$ are located at the sinus venosus or on the pericardial surface.

In this important time window, between $54 \mathrm{hpf}$ and $100 \mathrm{hpf}$, the very first communication between epicardium and myocardium is established. At later stages, epicardial cells undergo EMT and give rise to different epicardial-derived cells (EPDCs). In order to study the onset of this intercellular cross talk in zebrafish, I first aimed at generating different models of impaired epicardial coverage. Several models of impaired epicardial coverage have been described in mammals (see section 1.6.1; (Kwee et al., 1995; J. T. Yang et al., 1995; Moore et al., 1999; 
Gittenberger-de Groot et al., 2000; Manner et al., 2005; Acharya et al., 2012), but none are currently available in zebrafish.

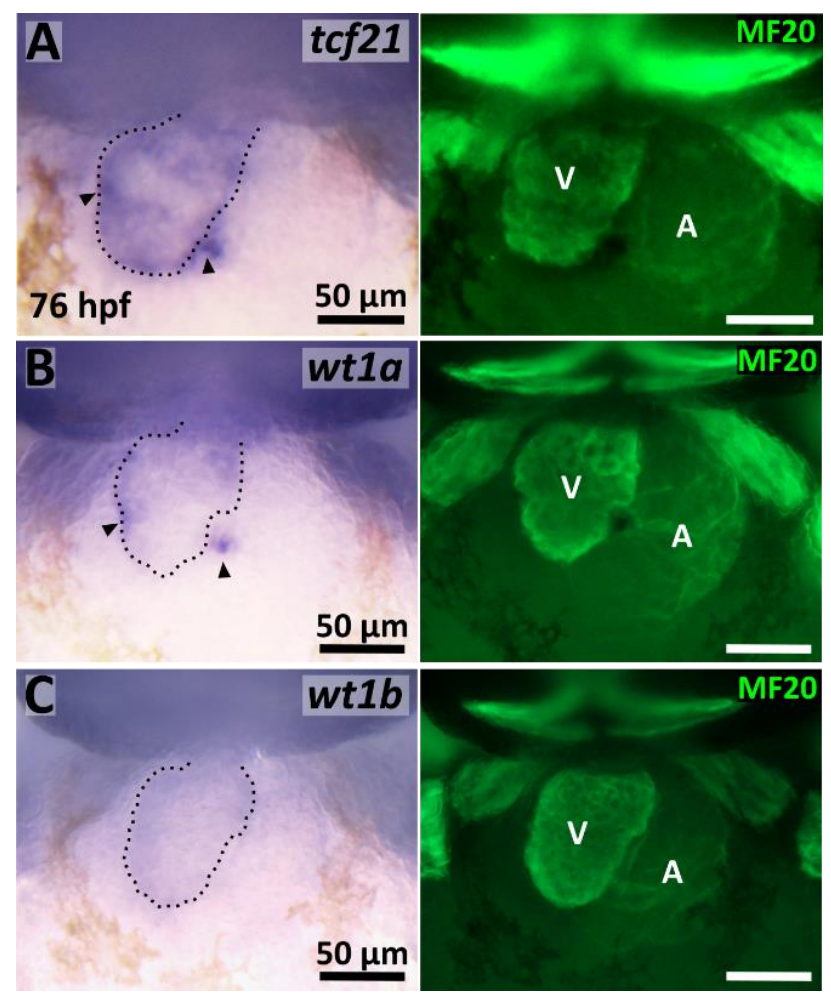

Figure 51: $t c f 21$ and $w t 1 a$ are expressed in the zebrafish epicardium at $76 \mathrm{hpf}$

A-C) In situ hybridization analyses of $\operatorname{tcf21(A),wt1~a~(B),~and~} w t 1 b$ (C) in 76 hpf hearts. On the right, MF-20 immunostaining (green), used to localize the atrial and ventricular chambers. The in situ staining for $\operatorname{tc} 21$ and $w t 1 a$ mRNAs (arrowheads) appears localized external to the myocardium (dashed lines).

Therefore, I first started with generating two zebrafish mutant lines. Among the well-known epicardial markers, I created CRISPR/Cas9 induced mutations in the transcription factor genes tcf 21 and wt1a. In mouse, lack of $T c f 21$ or $W t 1$ lead to impaired epicardial coverage, but the impact on early myocardial development is not yet fully understood (Moore et al., 1999, Acharya et al., 2012). In zebrafish, as in mice, these genes appear to be the most prominently expressed in the early epicardium (Serluca, 2008; J. Liu and Stainier, 2010; Peralta et al., 2014). I then confirmed their epicardial expression at $76 \mathrm{hpf}$ by performing in situ hybridization analyses (Figure 51A, B), while I could not appreciate any cardiac expression of $w t_{1} b$ (the paralog of $w t 1 a)$ at the same stage (Figure 51C).

For $t c f 21$, I recovered a 10 bp deletion in the first portion of the b-HLH DNA binding domain (exon 1). This mutation caused a frame-shift in the $\operatorname{tcf} 21 \mathrm{mRNA}$ sequence, which leads to the formation of a truncated protein missing the b-HLH domain and, therefore, its DNA binding ability (Figure 52). 


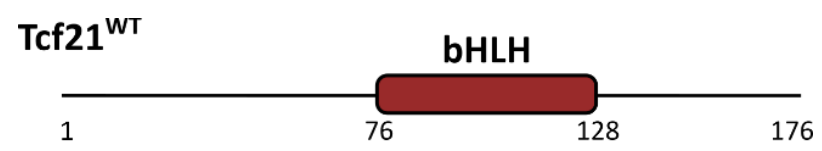

\section{Tcf21 ${ }^{\text {bns427 }}$}

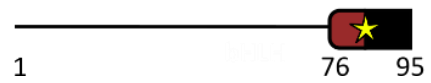

Figure 52: $t c f 21^{b n s 427}$ carry a $10 \mathrm{bp}$ deletion leading to the formation of a truncated protein

Wild-type and mutant Tcf21 proteins, highlighting in red the bHLH DNA binding domain and the amino acid positions. The yellow star refers to the CRISPR/Cas9-induced mutation site, while the black box indicates the missense segment following the frameshift lesion.

As previously mentioned, the zinc-finger transcription factor gene $W t 1$ has two orthologs in zebrafish, $w t 1 a$ and $w t 1 b$. Since paralog genes are known to frequently compensate for the lack of one another, I generated a deletion on the promoter region of $w t 1 a$, to prevent a potential transcriptional adaptation phenomenon. We injected two sgRNAs spanning an $8 \mathrm{~kb}$ region on the promoter of $w t 1$, including the first portion of the coding sequence (Figure 53A). After confirming the $8 \mathrm{~kb}$ deletion by PCR in F1 fish, I assessed $w t 1$ a mRNA expression in $w t 1 a^{+/+}$, $w t 1 a^{+/-}$, and $w t 1 a^{-/-}$larvae derived from $w t 1 a^{+/-}$intercrosses, at $96 \mathrm{hpf}$. In $w t 1 a^{-/-}$larvae, I observed the complete lack of mRNA, confirmed with two different couples of primers, one binding in the deleted portion of the gene and one targeting a downstream region, not affected by the DNA lesion. I also observed a $50 \%$ reduction of $w t 1 a \mathrm{mRNA}$ in $w t 1 a^{+/-}$larvae at the same stage (Figure 53B).

\section{A Wt1a ${ }^{\mathrm{WT}}$}

\section{Zn finger domains}

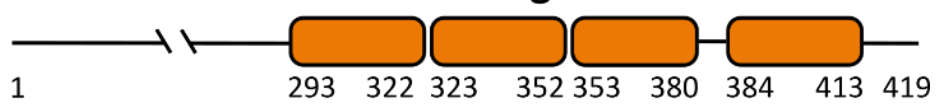

B

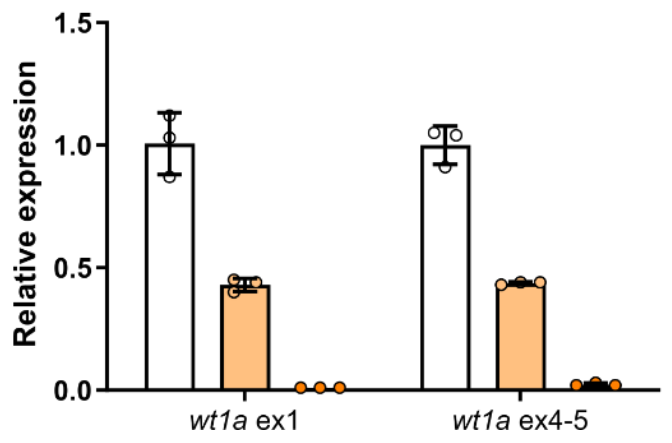

- $w t 1 a+/+$

- wt1a+/-

- wt1a-/-

Figure 53: wt1a promoter-less mutants lack the expression of wt1a mRNA

A) Schematic of the Wt1 a wild-type protein, showing the DNA-binding zinc finger domains, and their amino acid positions. B) $w t 1 a$ mRNA relative levels in $w t 1 a^{+/+}$, wt1 $a^{+/}$, wt1 $a^{/-} 96 \mathrm{hpf}$ larvae, as obtained by RTPCR. The two parts of the graph refer to different primers used to amplify $w t 1$ a cDNA, on exon1 (ex1; inside the genomic lesion) or in exon4-5 (ex4-5; outside the genomic lesion). Mean \pm SD. 
When I analyzed homozygous tcf 21 and wt1a larvae, I first observed the appearance of pericardial edema from $96 \mathrm{hpf}$ (Figure 54A-C). It is important to note that, for all the analyses, I always compared homozygous larvae with their siblings to avoid any influence of their different genetic backgrounds. However, to improve clarity and allow the comparison of the two mutant phenotypes, I represented the data combining the wild-type values, labeling them with different colors according to which experiment they were derived (red, $t c f 21^{+/}$- intercrosses; orange, $w t 1 a^{+/-}$intercrosses).

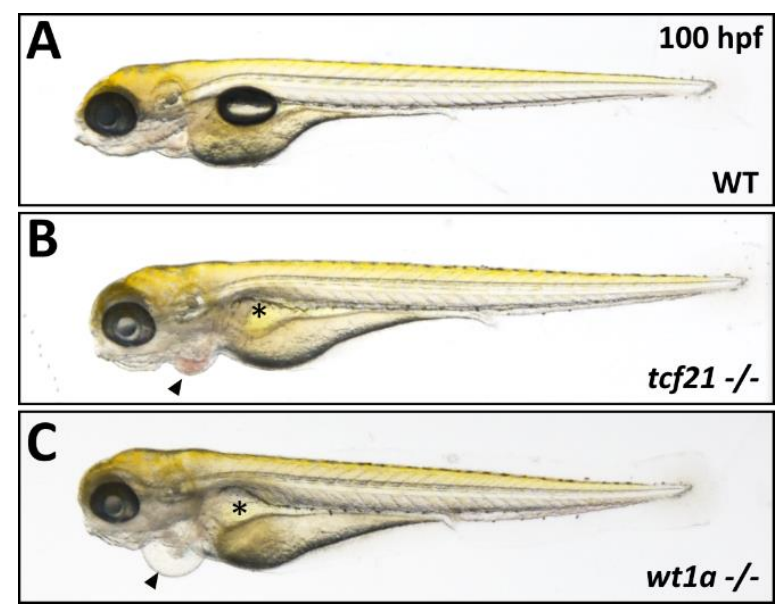

Figure 54: tcf21 and wt1a mutation cause pericardial edema in 96 hpf zebrafish larvae

A-C) Brightfield images of $96 \mathrm{hpf}$ wild-type (WT), tcf $21 \%$, and $w t 1 a^{\%}$ larvae. Both mutant larvae exhibit pericardial edema (arrowheads) and lack of swim bladder inflation (asterisks).

When I analyzed the epicardial coverage of the heart, I noticed a reduction in the number of epicardial cells attached to the ventricle, as it has been reported in mouse mutants (Moore et al., 1999; Acharya et al., 2012) (Figure 55A, B). To count the number of epicardial cells, I took advantage of the transgenic line $\operatorname{TgB} A C(\operatorname{tcf} 21: n l s-E G F P)$, which is known to label approximately all epicardial cells in larval and adult stages. In both mutants, the reduction in $\operatorname{tc} 21^{+}$cells attached to the ventricle started mildly at $54 \mathrm{hpf}$ and became more severe at 76 and $100 \mathrm{hpf}$ (Figure 55C). Remarkably, while $w t 1$ a mutants exhibit a complete absence of ventricular $\operatorname{tcf} 21^{+}$ cells, the reduction in epicardial coverage in tcf 21 mutants was highly variable, ranging from a complete loss to levels similar to wild types (Figure 55C). The phenotypic variability is likely not due to a hypomorphic allele, since the previously identified extra-cardiac defects of $\operatorname{tcf} 21$ mutants (i.e., lack of head muscles; Nagelberg et al., 2015; Burg et al., 2018) are consistently observed in all mutant larvae. 


\section{$\operatorname{Tg}($ myl7:mcherry-CAAX); $\operatorname{TgBAC}(\mathrm{tcf} 21$ :nls-EGFP)}
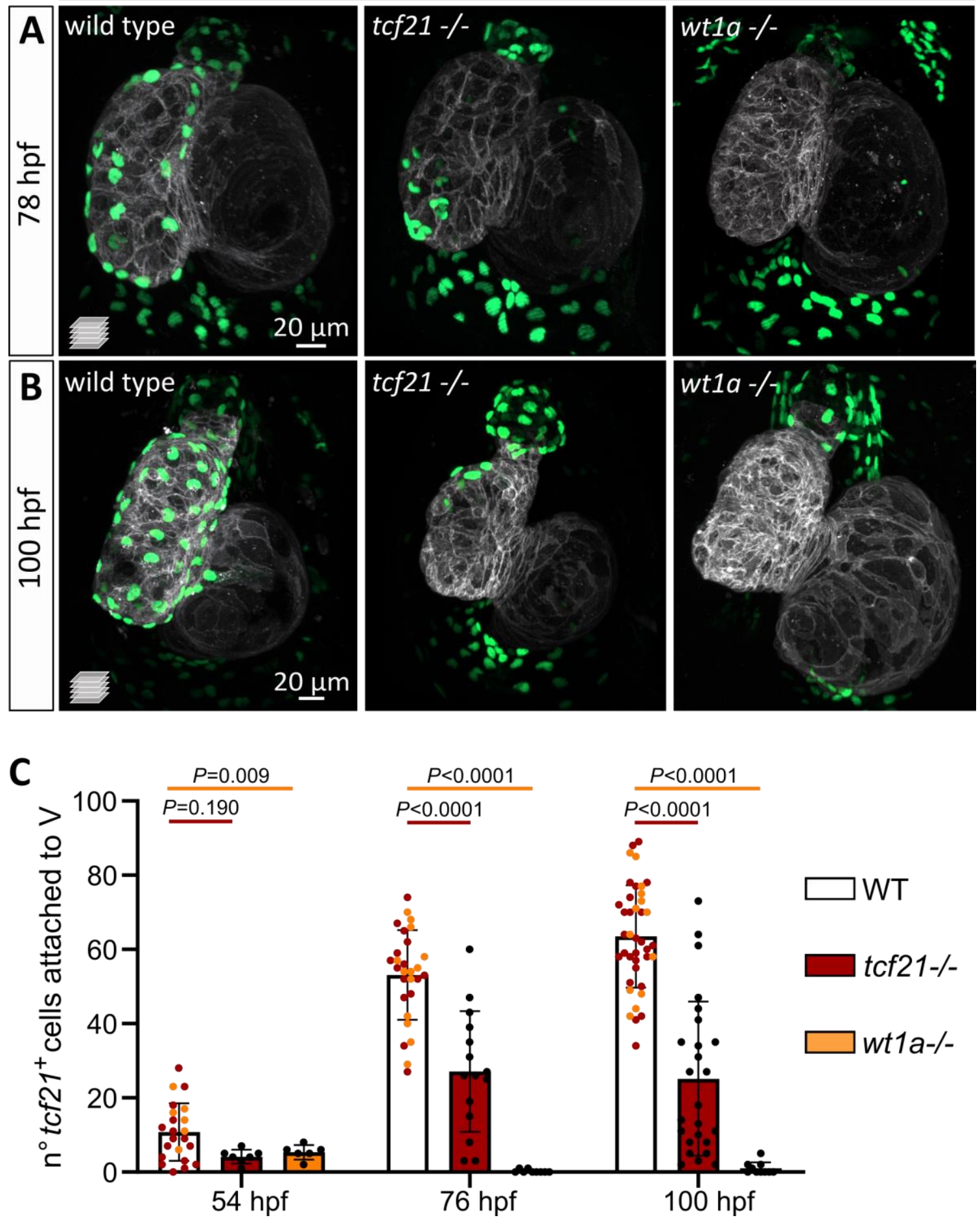

Figure 55: $t c f 21^{-/-}$and $w t 1 a^{-/-}$larvae exhibit reduced epicardial coverage of the ventricle

A-B) Confocal images of 76 (A) and 100 (B) hpf larvae. $t c f 21$ and $w t 1 a$ mutants exhibit reduced epicardial coverage compared with wild types at both stages analyzed. C) Quantification of $\operatorname{tcf} 21^{+}$cells attached to the ventricle at different developmental stages. The color of wild-type dots refers to $t c f 21 \%$ (red) or $w t 1 a^{-/}$ (orange) siblings. Mean $\pm \mathrm{SD} ; P$ values derived from $t$ - or Mann-Whitney tests (following normality tests), compared with the wild-type siblings of each genotype. 
Then, I wondered if this defect could be due to an impairment in the specification or in the attachment of epicardial cells to the ventricle. To assess that possibility, I counted the total number of $\operatorname{tcf} 21^{+}$cells in the pericardial region, including the cells still localized in the proepicardial organ. Interestingly, in $w t 1$ a mutants, the total number of $\operatorname{tcf} 21^{+}$was already reduced at $54 \mathrm{hpf}$, suggesting a defect in the early specification of these cells (Figure 56A). In contrast, $t c f 21^{\%}$ larvae did not show any difference in the total number of $t c f 21^{+}$cells compared with wild types at $54 \mathrm{hpf}$ or $73 \mathrm{hpf}$. Conversely, at $100 \mathrm{hpf}$, the number of $t \mathrm{cf} 21^{+}$was reduced in $\operatorname{tcf} 21 \%$ hearts compared with wild-type (Figure 56B). This decrease in the number of epicardial cells is likely caused by the death of the cells not attached to the ventricle. Several detached $\operatorname{tcf} 21^{+}$cells were labeled by the TUNEL assay at $85 \mathrm{hpf}$, therefore suggesting apoptotic or necrotic cell death (Figure 56C). Altogether, these results suggest a different defect causing the reduction of attached epicardial cells in the two different mutants. Notably, when I counted OFT tcf $21^{+}$cells, I did not observe any difference in $t c f 21^{--}$or $w t 1 a^{-/-}$larvae compared with wild types. This result is consistent with the different origin of this epicardial population, not deriving from the PEO (Perez-Pomares et al., 2003, Weinberger et al., 2020) (Figure 56D).
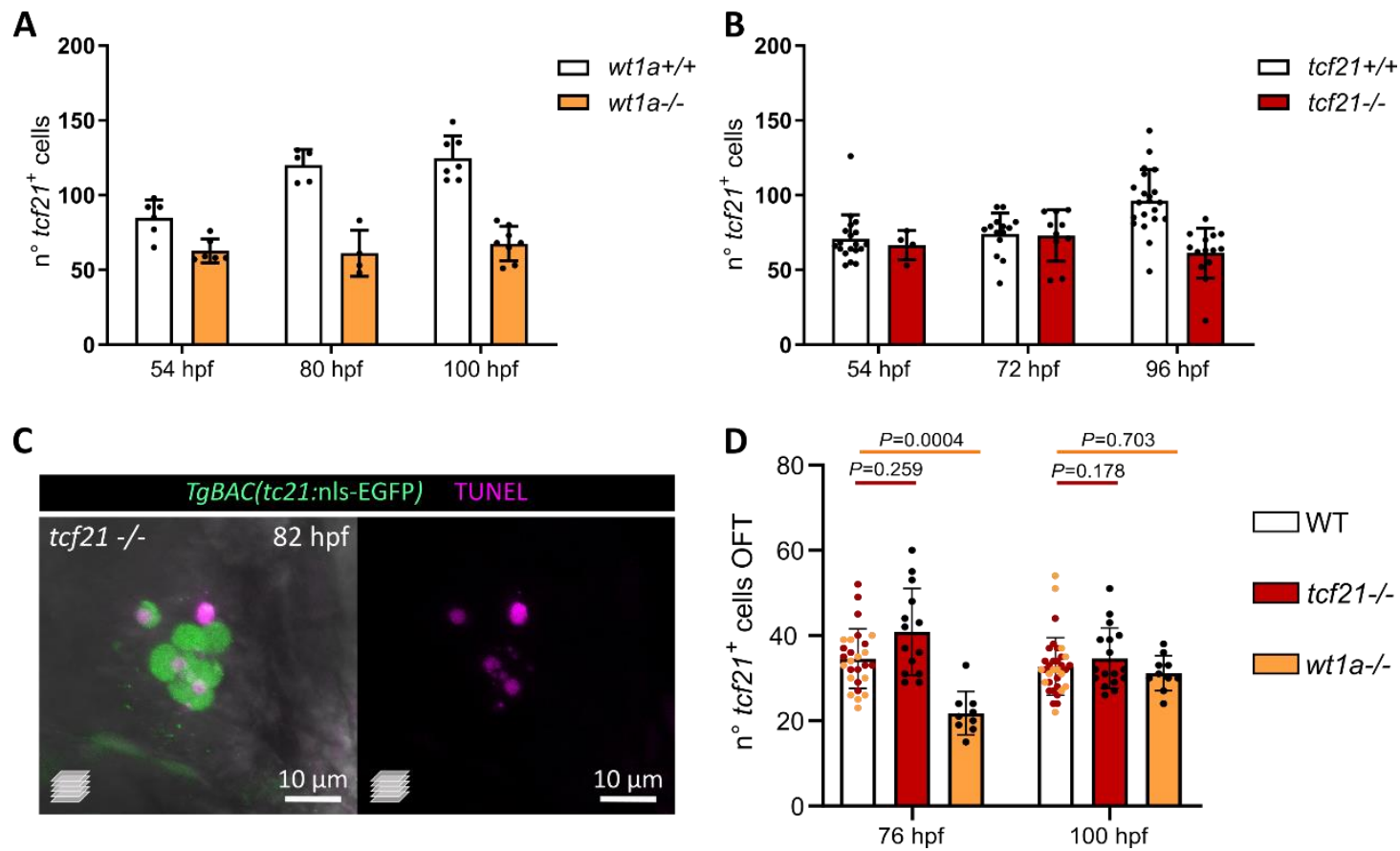

Figure 56: Not all $t c f 21^{+}$cells are equally affected in wt1a and $t c f 21$ mutants

A, B) Quantification of the total number of $t c f 21^{+}$cells in the pericardial region at different developmental stages, in $w t 1 a$ (A) or tcf21 (B) mutant larvae compared with wild types. C) Confocal images of 82 hpf tcf21/- hearts showing detached epicardial cells (green) positive for the TUNEL assay (magenta). D) Quantification of OFT $t c f 21^{+}$cells at different developmental stages, in $w t 1$ a or $t c f 21$ mutant larvae compared with wild types. The colors of wild-type dots refer to $t c f 21 \%$ (red) or $w t 1 a^{-/-}$(orange) siblings. Mean \pm SD; $P$ values from $t$ - or Mann-Whitney tests (following normality test), compared with the wild-type siblings of each genotype. 
To complement the study, I sought to obtain an "acute" model of impaired epicardial coverage, to achieve temporal-specific control of the process. To do so, I used the previously described nitroreductase/metronidazole (NTR/Mtz) system to ablate tcf $21^{+}$cells expressing TgBAC(tcf21:mCherry-NTR) (Nagelberg et al., 2015, Wang et al., 2015). The bacterial enzyme NTR specifically induces apoptosis upon administration of its substrate, Mtz. After performing the epicardial ablation at larval stages (from 52 to 100 hpf) (Figure 57A), I could phenocopy the absence of epicardial coverage observed in the two genetic models (Figure 57B), as well as their pericardial edema (data not shown).

A

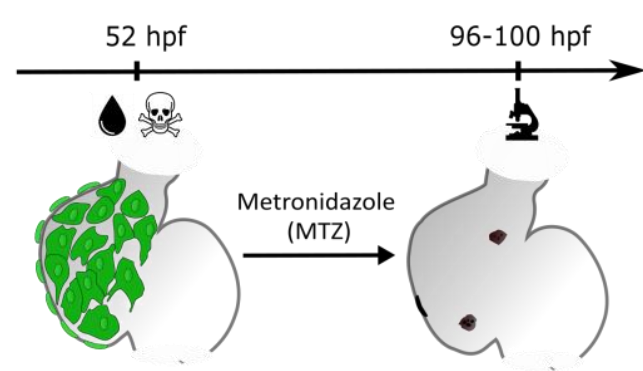

Tg(tcf21:mcherry-NTR)

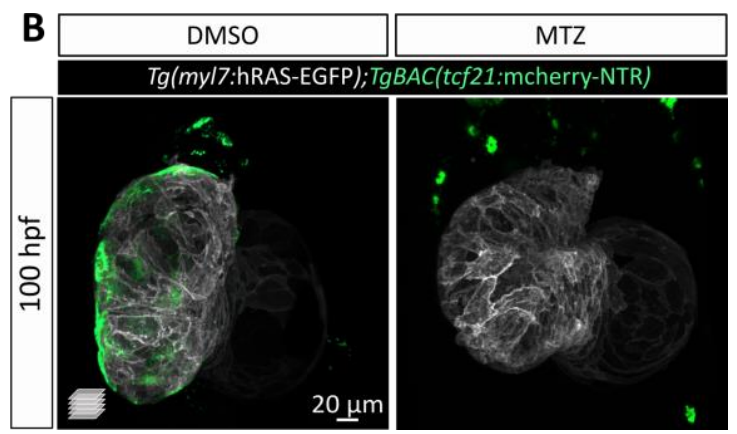

Figure 57: The NTR/Mtz system efficiently ablates epicardial cells in the zebrafish larvae

A) Schematic depicting the setting used for epicardial ablation. B) Confocal images of $100 \mathrm{hpf}$ $\operatorname{TgBAC(tcf21:mCherry-NTR);(myl7:bRAS-EGFP)}$ hearts, showing the result of epicardial ablation (MTZ), compared with DMSO-treated fish.

Lastly, I asked whether the lack of $t c f 21^{+}$cells in the three models would coincide with a complete absence of epicardial cells. Alternatively, it could be possible that spared epicardial cells attached to the ventricle would not express the $\operatorname{tcf} 21$ marker that allows to visualize them. To answer this question, I immunostained wild-type, $\operatorname{tc} 22^{\%}, w t 1 a^{-/}$, Mtz-treated larvae with the Caveolin1 antibody, a known pan-epicardial marker (Cao et al., 2016). Of note, all three models exhibited a lack of Caveolin1 immunostaining around the ventricle (except in correspondence with the remaining $\operatorname{tcf} 21^{+}$cells), but not in the OFT (Figure 58). This result provides further confidence about the lack of ventricular epicardial cells in the three models.

Altogether, these three partially independent models constitute a fundamental basis to study the effect of lack of epicardium on heart morphogenesis. They could also be instrumental for future research investigating the different mechanisms underlying the loss of epicardial coverage, as suggested by the differences in tcf21 and $w t 1 a$ mutants (Figure 56). 

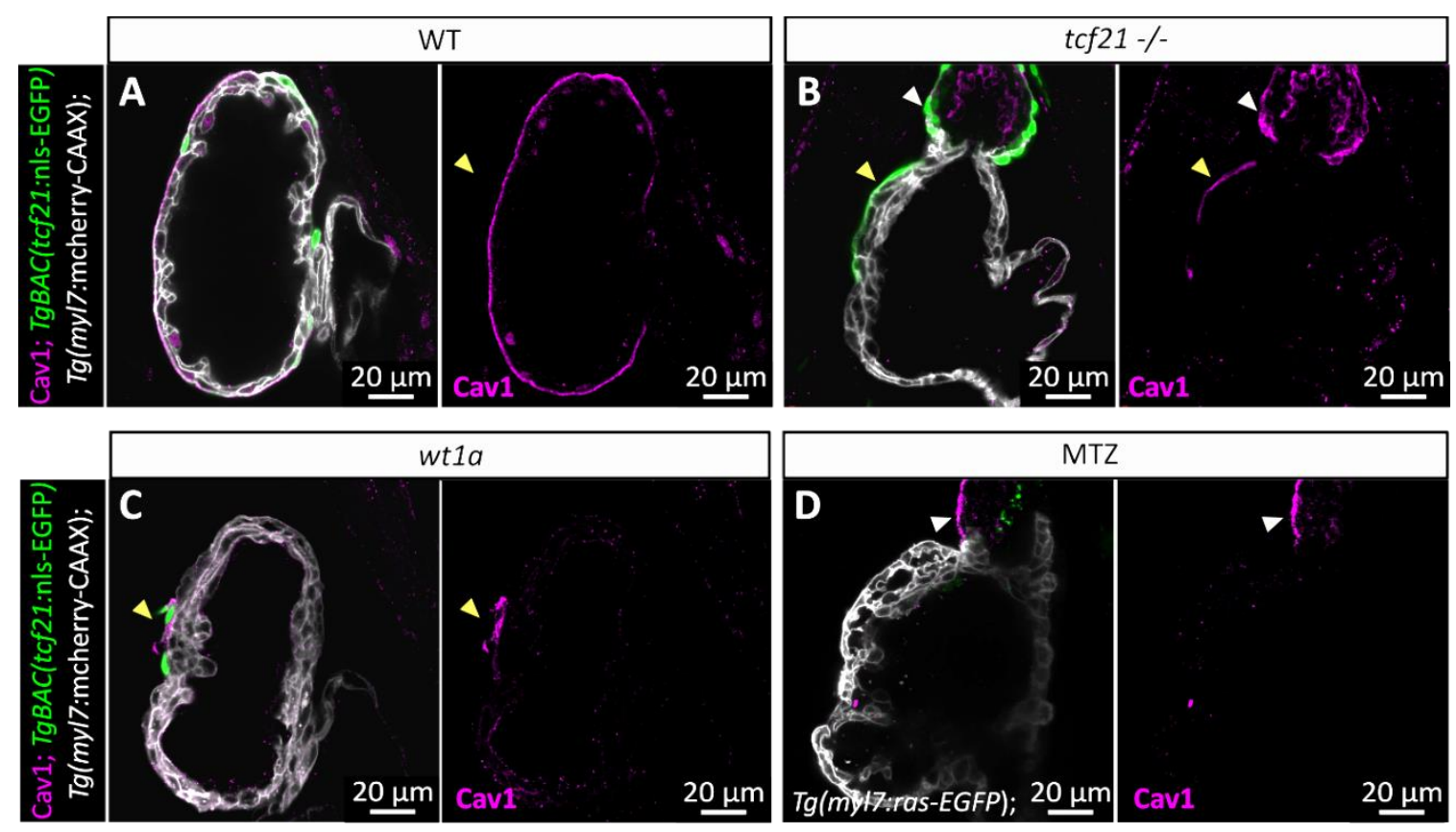

Figure 58: The lack of $t c f 21^{+}$cells correlates with the lack of $\mathrm{Cav1} 1^{+}$epicardial cells in the three models A-D) Confocal images of $100 \mathrm{hpf}$ hearts immunostained for Caveolin1 (Cav1), a pan-epicardial marker. The immunostaining for Cav1 colocalizes with the $\operatorname{TgB} A C$ (ttf21:nls-EGFP) expression, and it is absent from mutant and MTZ-treated hearts, with the exception of the spared tof $21^{+}$cells (yellow arrowheads). Conversely, Cav1 staining in the OFT does not appear affected (white arrowheads).

\subsubsection{Lack of epicardial coverage leads to impaired ventricular growth without affecting cardiomyocyte number}

Lack of or reduced epicardial coverage of the heart has been associated with cardiac defects during development, among which the reduced cardiac size and ventricular non-compaction are the most common (Sucov et al., 1994, Kwee et al., 1995, Yang et al., 1995, Moore et al., 1999, Pennisi et al., 2003, Manner et al., 2005, Acharya et al., 2012). To date, no causing factor is unanimously accepted to explain these phenotypes, and the precise role of the epicardium in promoting cardiac development is still unclear. Therefore, I aimed to understand if the lack of epicardium in the three zebrafish models would affect ventricular morphogenesis. First, I observed the formation of pericardial edema starting from $96 \mathrm{hpf}$ in $t c f 21^{-/,}, w t 1 a^{-/-}$and $\mathrm{NTR}^{+}$ Mtz-treated larvae, suggesting impaired cardiac function. Therefore, I analyzed the heart rhythm at 72 and $96 \mathrm{hpf}$ and did not find any major impairment in any of the mutants (Figure 59A). Notably, I observed reduced ventricular fractional shortening (a proxy for cardiac contraction) at $96 \mathrm{hpf}$ in all the three models compared with wild types (Figure 59B). 

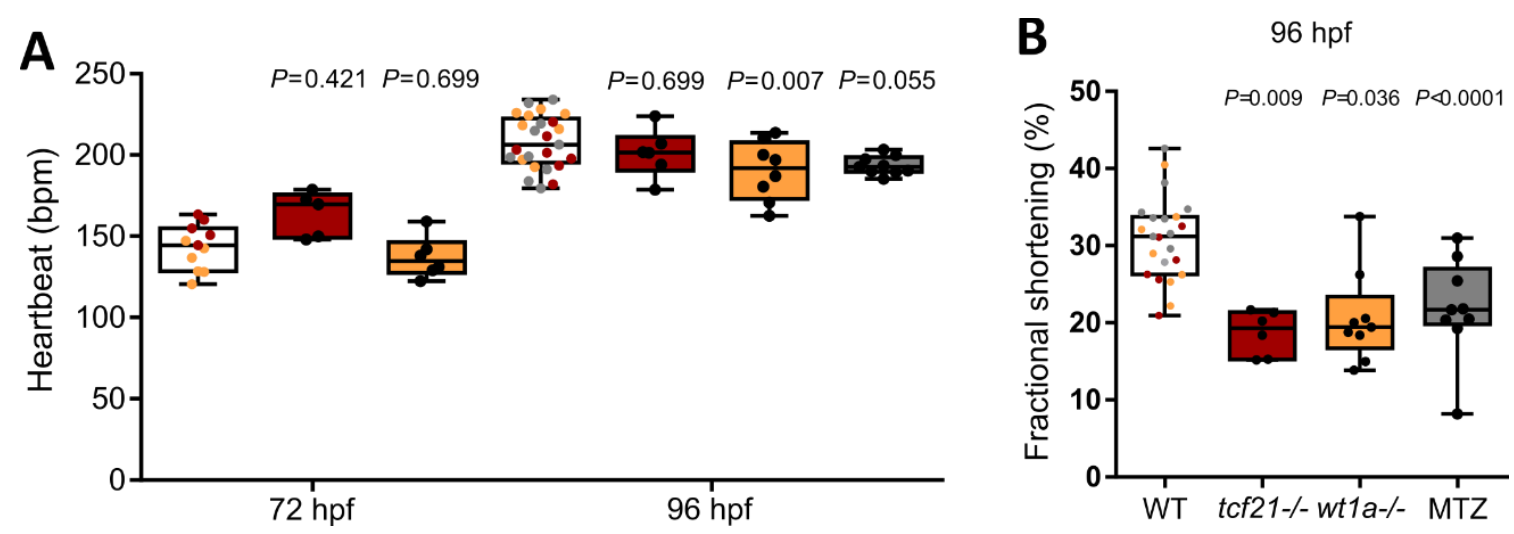

Figure 59: Mutant and Mtz-treated larvae exhibit impaired contractility at $96 \mathrm{hpf}$

A-B) Quantification of heart rate (A) and fractional shortening (B) in 72 and 96 hpf wild-type, mutant and Mtz-treated larvae. The colors of wild-type dots refer to tcf $21 \%$ (red), wt1 $a^{\%}$ - (orange) or Mtz-treated (grey) siblings. Mean $\pm \mathrm{SD} ; P$ values from $t$ - or Mann-Whitney tests (following normality test), compared with the wild-type/control siblings of each genotype/treatment.
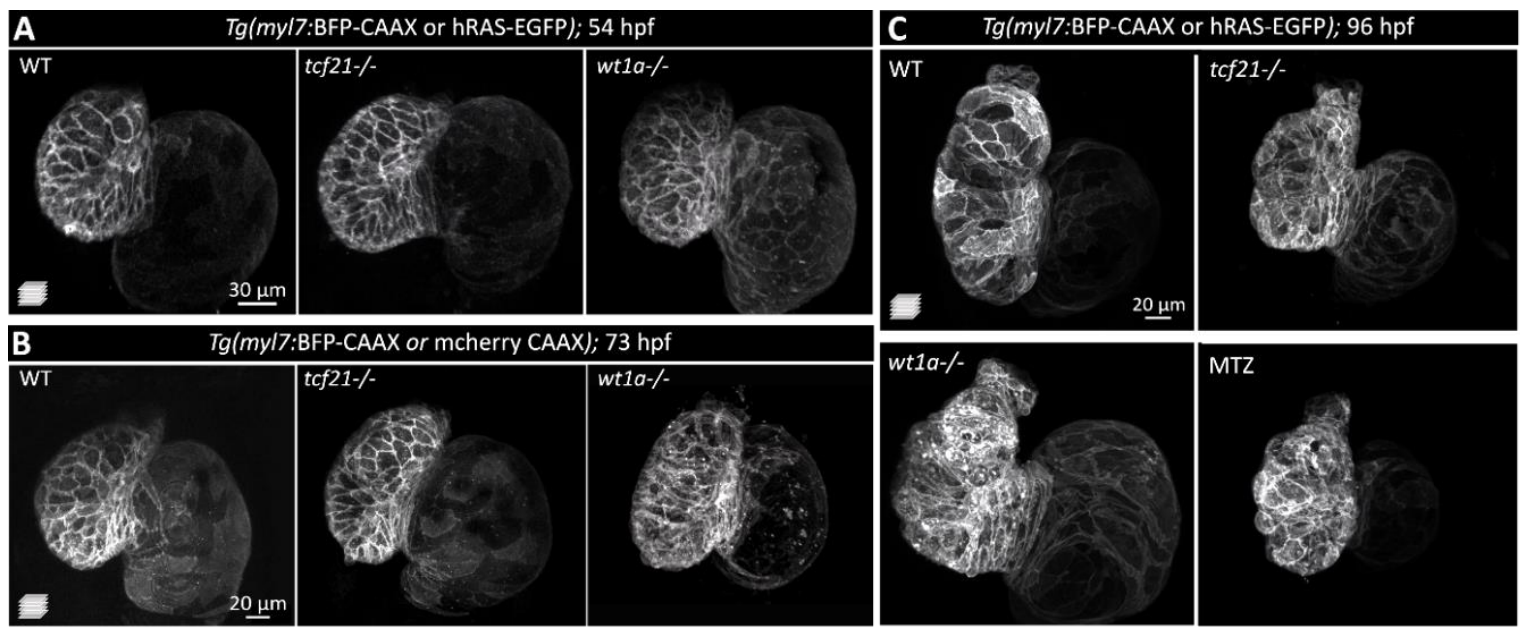

Figure 60: tcf 21 and wt1a mutants exhibit a smaller ventricle starting at $96 \mathrm{hpf}$

A-C) Confocal images of wild-type, $t c f 21, w t 1 a$, and Mtz-treated larvae at 54 (A), 73 (B), and 96 (C) hpf. White, myocardial membranes labelled by different transgenes. In A and B, mutant larvae do not exhibit any obvious morphological alteration, while in $\mathrm{C}$ they display a reduced ventricular size.

When looking at the morphology of 100 hpf hearts, I observed that the size of the ventricle appeared smaller in all three models (Figure 60C). Of note, $\operatorname{tcf} 21 \%$ and $w t 1 a^{-\%}$ hearts looked comparable to wild types at earlier stages (54 and 73 hpf) (Figure 60A, B).

In order to investigate the processes involved in this cardiac phenotype, I first asked whether and to what extent the ventricular volume increases in wild-type fish during larval stages. After measuring the ventricular volume in live animals, I observed a significant increase in its size $(+36.4 \%)$ between 72 and 100 hpf (Figure 61A, B). Conversely, $t c f 21^{\%}$ and $w t 1 a^{-/}$larvae exhibited a comparable ventricular volume at $72 \mathrm{hpf}$, while their ventricles failed to significantly enlarge over time $\left(\operatorname{tcf} 21^{-\%},-8.9 \%\right.$; $\left.w t 1 a^{-/}+9,8 \%\right)$ (Figure 61A, B). 
A

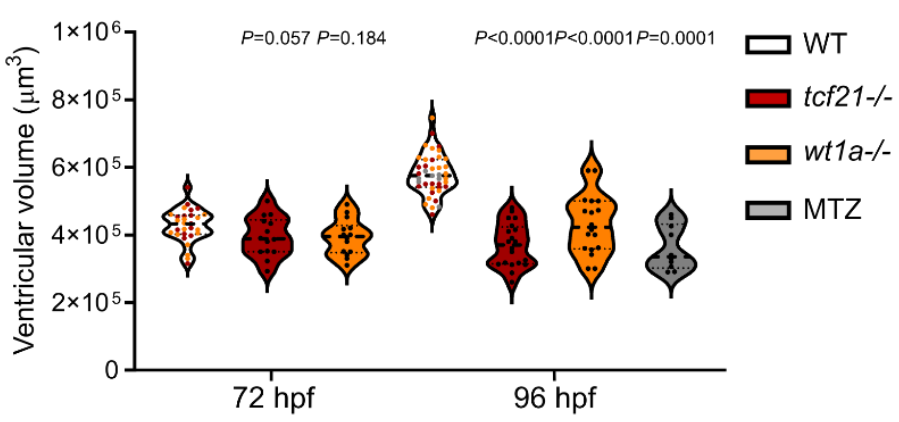

B

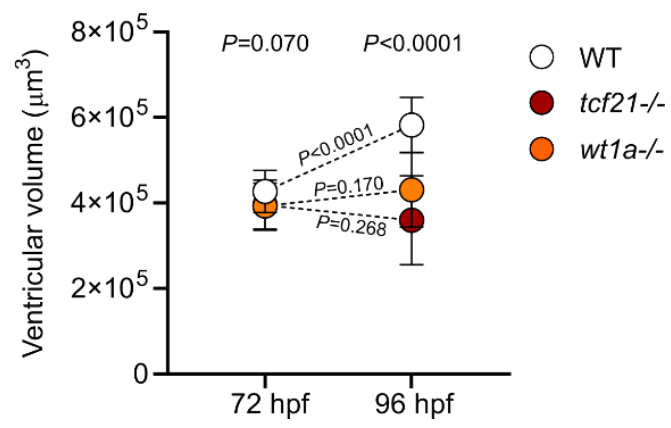

Figure 61: $t c f 21^{-/-}$and $w t 1 a^{-/-}$ventricle fail to grow over time as the wild-type.

A) Quantification of ventricular volume in 72 and $96 \mathrm{hpf}$ larvae. The colors of wild-type dots refer to $t c f 2^{-/-}$ (red), $w t 1 a^{-/}$(orange) or Mtz-treated (grey) siblings. Mean \pm SD; $P$ values from $t$ - or Mann-Whitney tests (following normality test), compared with the wild-type/control siblings of each genotype/treatment. B) Representation of the ventricular volume variation over time in WT, $t c f 21$, and $w t 1$ a mutant larvae. Mean \pm SD; $P$ values from One-Way ANOVA, among the three different genotypes at the same time-point, or $t$-tests, within the same genotype comparing the two different time-points.

The increase in an organ size can be driven by hyperplastic and/or hypertrophic growth, depending if it is directed by an increase in cell number or cell size, respectively. Proliferation has proven to be essential for proper cardiac growth (Uribe et al., 2018). Therefore, I first analyzed the number of ventricular CMs at 72 and $96 \mathrm{hpf}$, which increased from $169.7 \pm 13$ to $219.2 \pm 19$ in W'T fish (+29.6\%) (Figure 62A, B). Notably, CM number similarly increased over time also in $w t 1 a^{-/}$and $t c f 21^{\%}$ larvae and was not significantly different from WT at any time-point analyzed (Figure 62A, B). To further elucidate the contribution of a proliferation defect to the reduced ventricular volume, I took advantage of the Tg(myl7:venus-gmnn) transgenic line, which labels CMs entering the cell cycle (Uribe et al., 2018) (Figure 62C).

With this tool, I detected a similar percentage of proliferating CMs in DMSO and MTZ treated larvae at $80 \mathrm{hpf}$ (Figure 62D), supporting also the comparable number of CMs observed at 96 hpf (Figure 62A). These data suggest that hyperplastic growth between 72 and $96 \mathrm{hpf}$ is not affected in the absence of epicardium.

Figure 62: $t c f 21 /$-, wt1a/- or Mtz-treated hearts do not exhibit defects in CM number (next page) A) Quantification of ventricular CM number in 72 and $96 \mathrm{hpf}$ larvae. The colors of wild-type dots refer to tcf $21 \%$ (red), wt1 $a^{/-}$(orange) or Mtz-treated (grey) siblings. Mean \pm SD; $P$ values from $t$ - or Mann-Whitney tests (following normality test), compared with the wild-type/control siblings of each genotype/treatment. B) Representation of the ventricular CM number variation over time in WT, tcf21, and wt1 a mutant larvae. Mean \pm SD; $P$ values from One-Way ANOVA, among the three different genotypes at the same time-point, or $t$-tests, within the same genotype comparing the two different time-points. C-D) Confocal images and quantification of myl7:mVenus-gmnn ${ }^{+} \mathrm{CMs}$ in $80 \mathrm{hpf}$ MTZ-treated control larvae (-NTR) and

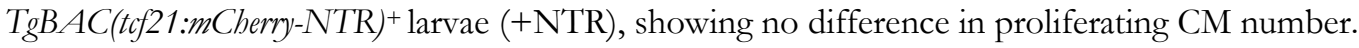


A

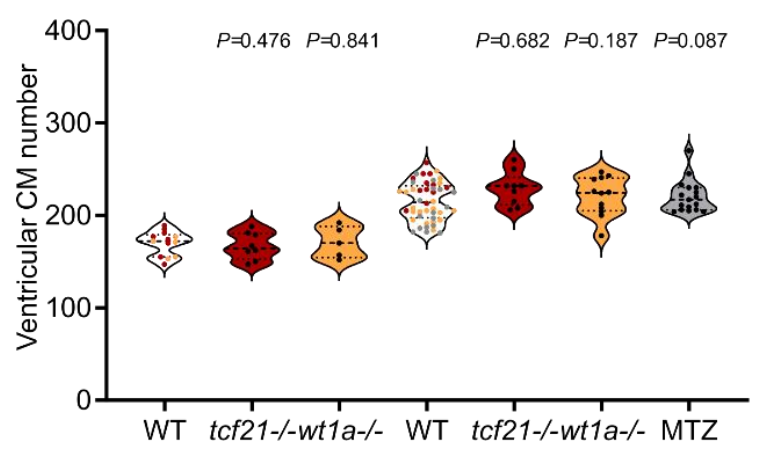

C

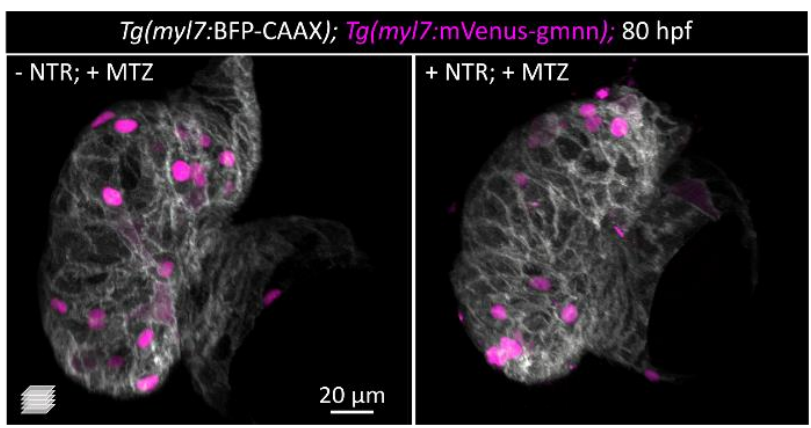

B

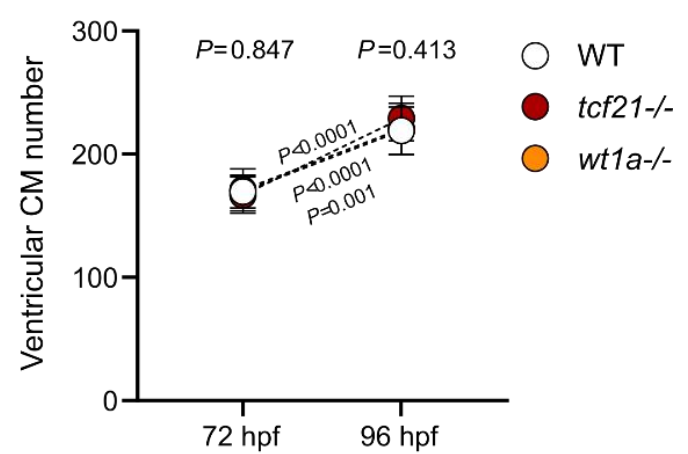

D

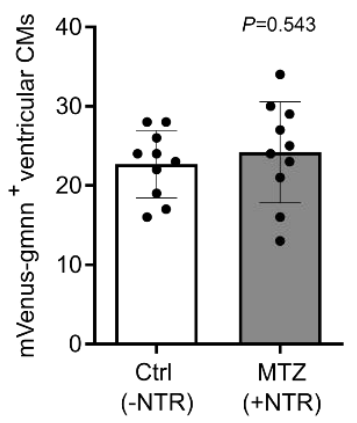

\subsubsection{Lack of epicardial coverage leads to impaired ventricular size increase}

After determining that the defect in ventricular volume was not due to a reduced CM number, I aimed at analyzing cellular size. At first, I observed that the average distance between CM nuclei was reduced in $96 \mathrm{hpf} t c f 21^{\%}, w t 1 \mathrm{a}^{-/}$, and Mtz- treated larvae compared with controls (Figure 63A-C). Considering the lack of extracellular space between CMs, this result suggests a reduced cellular size compared with wild-type.

A
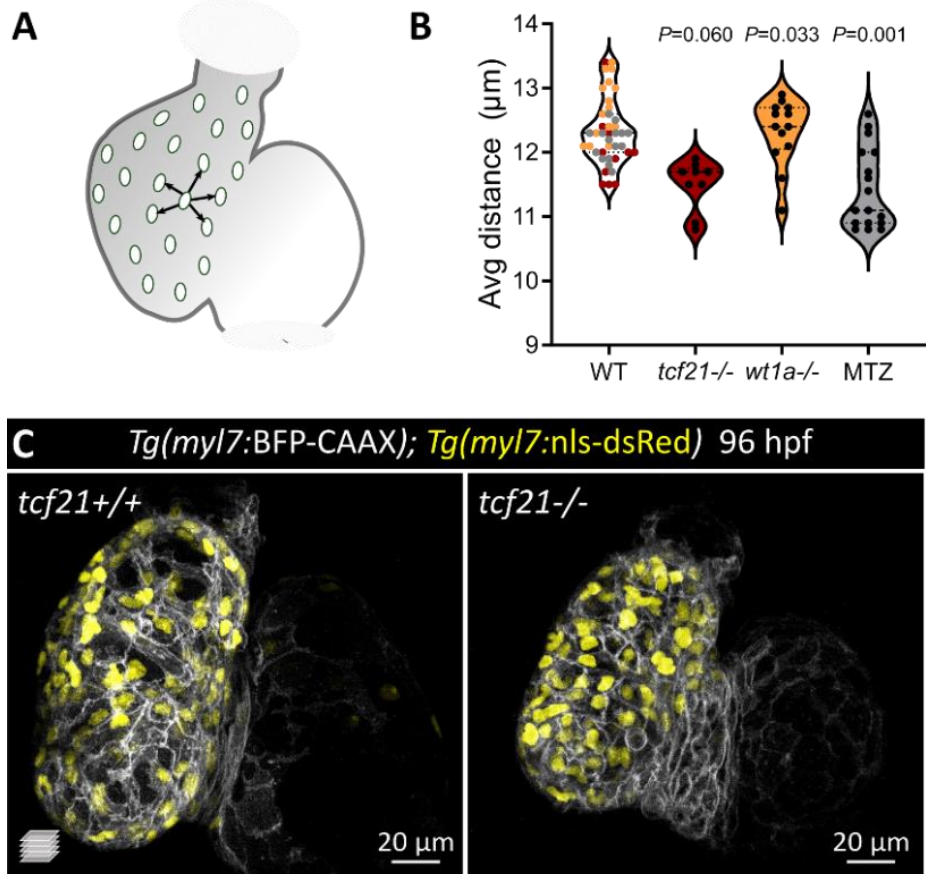

Figure 63: Lack of epicardium affects $\mathbf{C M}$ distance

A) Schematic depicting the "average nuclear distance" analyses, which calculates the average distance from the 5 closest neighboring nuclei. B) Quantification of the average distance between CM nuclei in $96 \mathrm{hpf}$ larvae. The colors of wild-type dots refer to $t c f 21 \%$ (red), $w t 1 a^{\%}$ - (orange) or Mtz-treated (grey) siblings. Mean \pm SD; $P$ values from $t$ - or MannWhitney tests (following normality test), compared with the wildtype/control siblings of each genotype/treatment. C) Confocal images showing reduced CM nuclear (yellow) distance in tof $21 \%$ hearts, compared with $t c f 21^{+/+}$hearts. 
To assess CM size, I first quantified the apical area of compact layer CMs on the ventricular surface. While at $72 \mathrm{hpf}$ the average CM area was comparable in wild-type and mutant larvae, at 96 hpf mutant CMs were significantly smaller than wild-type (Figure 64A). Notably, the wild-type $\mathrm{CM}$ area increased over time (+73\%), while the mutant $\mathrm{CM}$ area did not (Figure 64B).

$w t 1 a-/-$
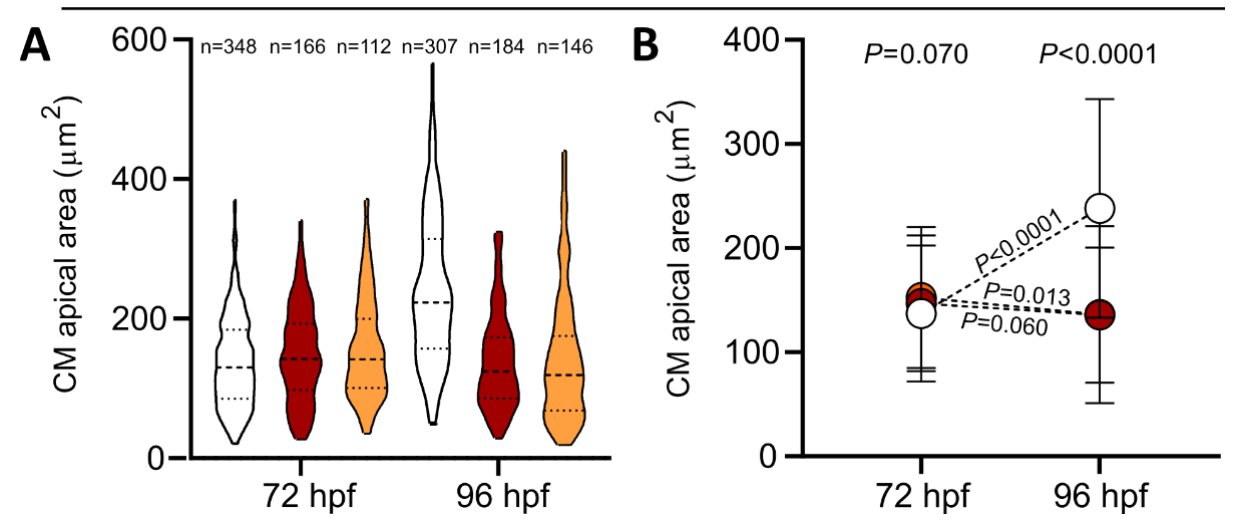

Figure 64: $t c f 21$ and wt1a mutants exhibit reduced $\mathrm{CM}$ apical area on the ventricular surface

A-B) Quantification of ventricular CM area distribution (A) and variation over time (B) in 72 and $96 \mathrm{hpf}$ larvae. Mean \pm SD. A) $P$ values from $t$ - tests, compared with the wild-type siblings of each genotype. B) $P$ values from One-Way ANOVA, among the three different genotypes at the same time-point, or $t$-tests, within the same genotype comparing the two different time-points.

Nonetheless, the reduced apical area could also reflect a defect in CM shape and orientation. To assess more precisely CM growth, I took an alternative approach, thanks to the zebrafish amenability to live imaging. I injected the myl7:mScarlet plasmid in embryos deriving from an intercross of $t c f 1^{+/}$fish. With this tool, injected larvae would mosaically express the construct in few CMs, allowing us to track single cells and measure their volume over time (Figure 65A). Considering the difference in CM shape and size depending on their position in the ventricle (Auman et al., 2007), I focused on CMs located on the outer curvature and the ventricular apex and excluded inner curvature and AV canal CMs from the analyses. I imaged the same larvae at 76 and $100 \mathrm{hpf}$ and observed a $25.7 \%$ increase in the volume of wild-type CMs between these two stages. Conversely, $\operatorname{tc} 221^{\%} \mathrm{CM}$ volume did not change in the same time window $(+1.4 \%)$ (Figure 65B). 

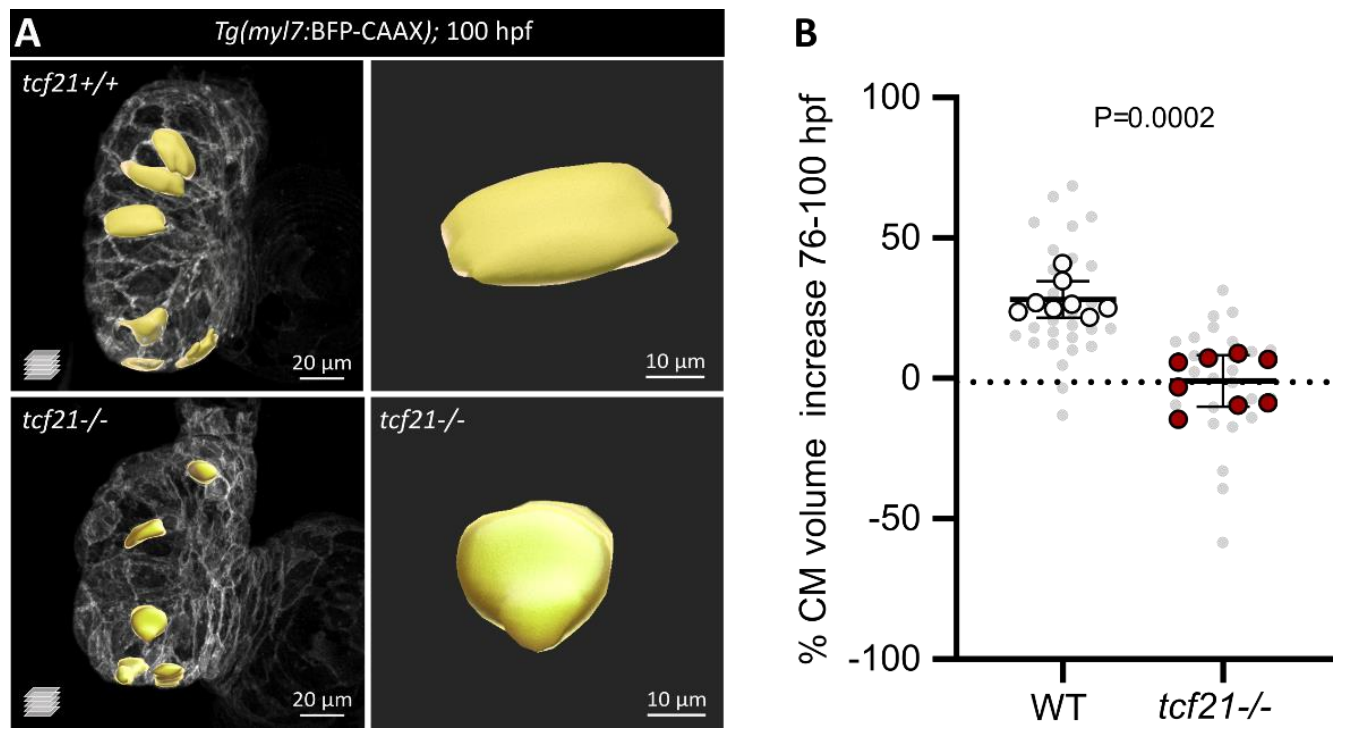

Figure 65: The tcf21 mutation leads to impaired growth of ventricular CMs

A) Confocal images and $3 \mathrm{D}$ reconstruction of single $\mathrm{CM}$ volume (yellow) in $96 \mathrm{hpf} t \mathrm{tf} 21^{+/+}$and $t \mathrm{tc} 2 \mathrm{r}^{1 /}$ hearts. B) Quantification of the \% volume increase of single CM between 76 and $100 \mathrm{hpf}$ in $\operatorname{tcf} 21^{+/+}$and $\operatorname{tc} 21 \%$ hearts. Grey dots, single CMs; white/red dots, average of the measured CMs per larva. $P$ value from $t$-test, comparing the averages per larva between the two genotypes.

In addition to the phenotypes described, I observed a significant number of CMs extruding from the abluminal myocardial wall in all three models (Figure 66A-E'). This phenotype started mildly at $76 \mathrm{hpf}$ and became more severe at $100 \mathrm{hpf}$ (Figure 66F), and it could be best observed when reconstructing the myocardial surface with the Imaris software (Bitplane) (Figure 66A-D). CM extrusions have been observed previously in a few cardiovascular mutants, and their cause has been linked to different mechanisms that impair myocardial wall development (Auman et al., 2007; Rasouli et al., 2018; Gentile et al., 2020). In the absence of the epicardium, I speculate that the crowding of CMs described above (Figure 63) leads to their extrusion from the layer. This process has extensively proven to be essential in both embryos and cell culture to reduce overcrowding without altering tissue integrity (Kocgozlu et al., 2016; Priya et al., 2020; Villars and Levayer, 2020).

Altogether, our observations propose an early role for the epicardium during ventricular development in facilitating CM volume increase. Notably, so far studies have mostly focused on the contribution of the epicardium to the hyperplastic growth of the heart by promoting CM proliferation (Sucov et al., 1994, Pennisi et al., 2003) or by forming different cell types via EMT (Moore et al., 1999, Mahtab et al., 2009, Acharya et al., 2012). Nonetheless, our data are not in contrast with these studies. In fact, I speculate that the impairment in cell growth might cause a subsequent defect in cell division. As it was shown in several other contexts, the dependency of cell cycle progression on growth is a well-established phenomenon in eukaryotic cells (Jorgensen and Tyers, 2004). Moreover, I speculate that during later cardiac development, 
impairment of epicardial coverage will lead to reduced EPDCs formation, which will further exacerbate the difference in cardiac volume compared with wild types.
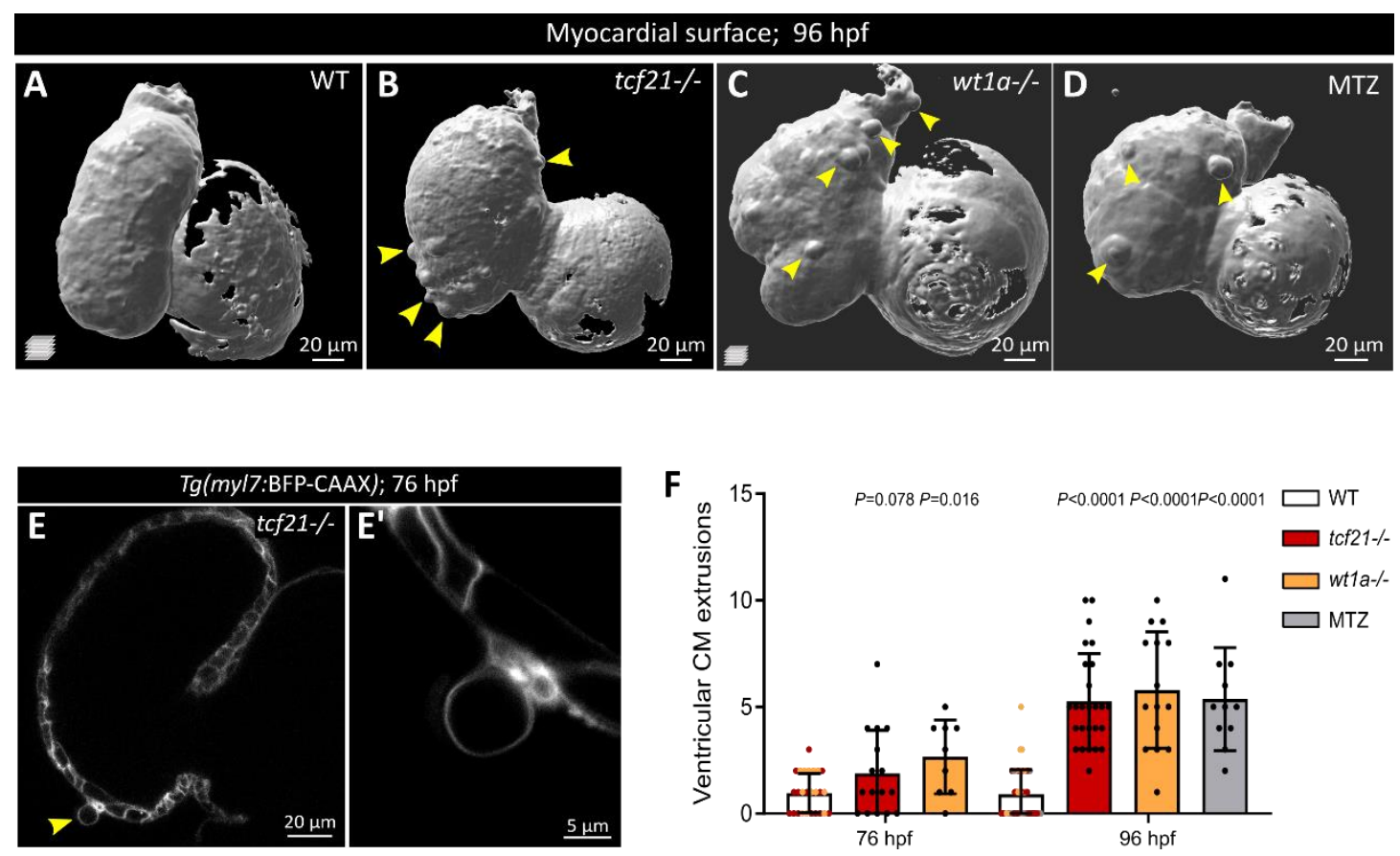

Figure 66: Impaired epicardial coverage causes $\mathrm{CM}$ abluminal extrusion from the ventricular wall A-D) 3D myocardial surface (Imaris reconstruction) from confocal images of $96 \mathrm{hpf} T g(m y l 7: m C h e r r y-C A A X)$ (A-C) or $\operatorname{Tg}(m y l 7: h R A S-E G F P)(D)$ hearts. Arrowheads point to CM extrusions. E, E') Cross-section of the myocardial wall in $76 \mathrm{hpf} \mathrm{Tg}(\mathrm{my} / 7: \mathrm{m} C h e r r y-C A A X) t c 21 \%$ heart. Arrowhead point to CM extrusions, also shown in E'. F) Quantification of CM extrusions at 76 and 96 hpf. The colors of wild-type dots refer to tcf $21 \%$ (red), $w t 1 a^{\%}$ (orange) or Mtz-treated (grey) siblings. Mean \pm SD; $P$ values from $t$ - or Mann-Whitney tests (following normality test), compared with the wild-type/control siblings of each genotype/treatment.

\subsubsection{Identification of a temporal window crucial for the role of the epicardium in cardiac development}

To further support the dependency of the impaired cardiac growth on the lack of epicardium, I took advantage of the temporal versatility of the NTR/MTZ system. Specifically, I first asked whether rescuing the epicardial coverage would be sufficient to improve ventricular growth. To do so, I relied on the ability of the epicardium to reactivate and regenerate after injury, thoroughly described during adult cardiac regeneration (Cao and Poss, 2018) and briefly shown during larval stages (J. Wang et al., 2015). I first ablated the epicardium as described before from 54 to $100 \mathrm{hpf}$ and, after assessing full epicardial ablation, I removed the compound from the water, allowing the fish to develop until $6 \mathrm{dpf}$ (hereafter referred to as "Mtz wash-out") (Figure 67A). I first confirmed the ability of the epicardium to regenerate during larval stages, likely by reactivation of spared epicardial cells. In most of the Mtz-treated larvae, the epicardium covered between 30 and $80 \%$ of the ventricle at $6 \mathrm{dpf}$ (Figure 67B, C). Surprisingly, I observed that the regeneration of the epicardium was sufficient to ameliorate 
cardiac growth (Figure 67B', C'). When I quantified the ventricular volume I observed a significant increase compared to larvae treated with Mtz from $54 \mathrm{hpf}$ to $6 \mathrm{dpf}$, and a volume similar to wild-types $(P=0.050)$ (Figure 67D). Moreover, the recovery of the epicardium also reduced the number of CM extrusions (1.3 \pm 1.5 ; DMSO: $0.5 \pm 0.7)$, compared with the average observed at 96 hpf in Mtz-treated larvae (5.4 \pm 2.4 ) (Figure 67E-G).

A

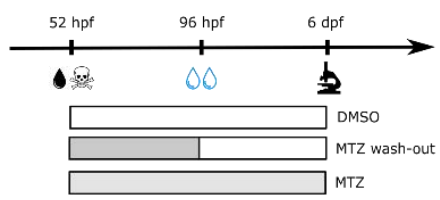

D

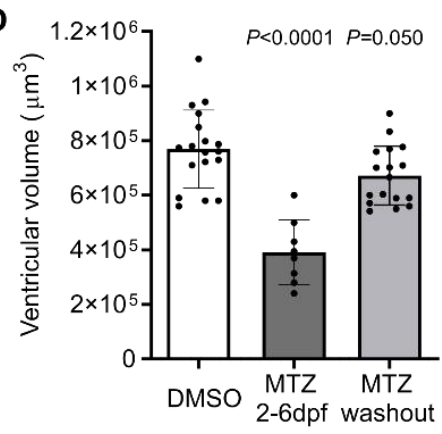

$\mathbf{E}$

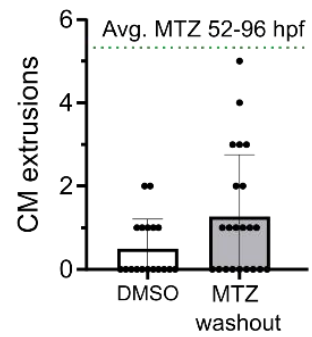

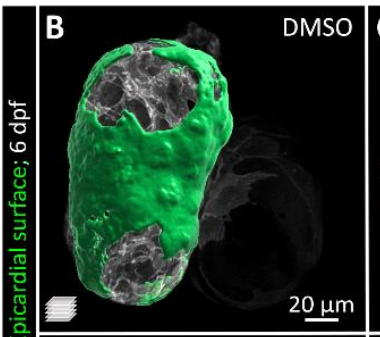
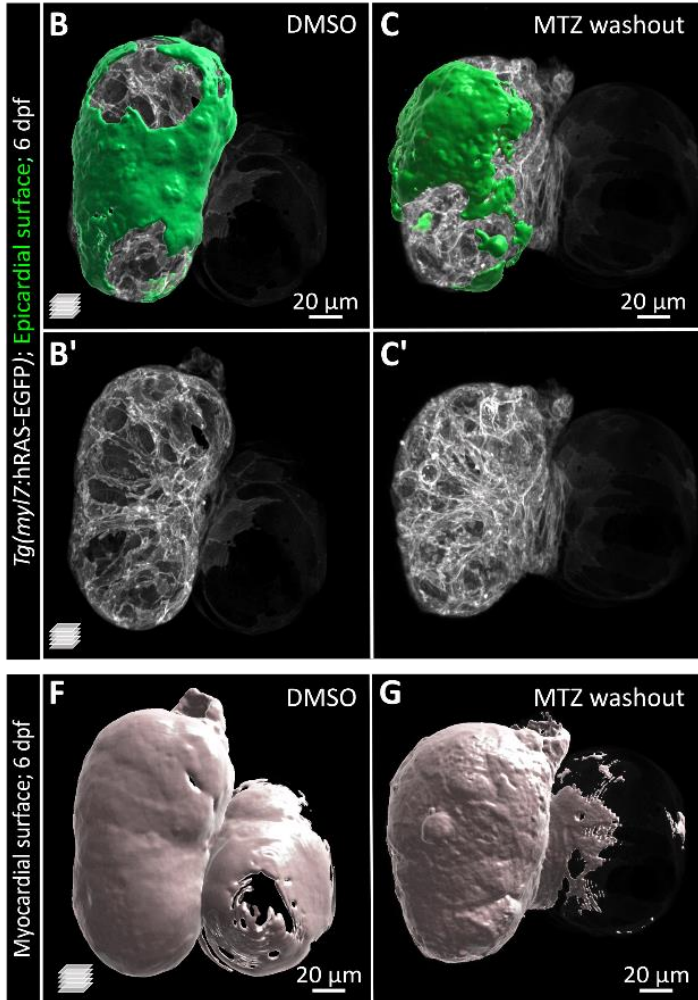

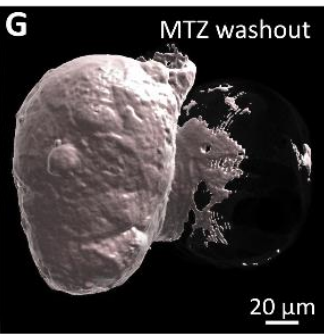

Figure 67: Epicardial regeneration after ablation rescues myocardial defects

A) Schematic of the Mtz-treatment setting used for this experiment. Mtz-washout refers to the removal of the compound at $4 \mathrm{dpf}$ and the recovery of the larvae until $6 \mathrm{dpf}$. B-C') Confocal images of $6 \mathrm{dpf}$ $\operatorname{Tg}(m y / 7: h R A S-E G F P) ; T g B A C(t c f 21: m C h e r r y-N T R)$ DMSO and Mtz-treated larvae. Green, 3D reconstruction of epicardial coverage, showing a partial regeneration in Mtz-washout larvae. D-E) Quantification of ventricular volume (D) and CM extrusions (E) at $6 \mathrm{dpf}$. The quantification of CM extrusions is compared with the average observed with the $52-96 \mathrm{hpf}$ Mtz treatment. F, G) Reconstruction of the myocardial surface of the hearts shown in $\mathrm{B}, \mathrm{C}$, exhibiting no evidence of $\mathrm{CM}$ extrusion.

All the previous studies describing reduced epicardial coverage in embryonic stages relied either on genetic models (in mammals; e.g., (Kwee et al., 1995; J. T. Yang et al., 1995; Moore et al., 1999; Mahtab et al., 2009) or on physical ablation of the early PE (in chick; e.g., (Pennisi et al., 2003; Manner et al., 2005; Takahashi et al., 2014). These models achieved a constitutive lack of epicardium from very early developmental stages, failing to provide temporal-specific information on its role in cardiac development. Moreover, most of the analyses performed on the published models focus on a time-point following the EMT process, further complicating the interpretation of the results. Therefore, with the use of the temporal-specific Mtz ablation 
model, I assessed the temporal window in which the epicardium is essential for cardiac growth during larval stages, preceding the onset of EMT. I previously identified the temporal window 52-96 hpf to be crucial for epicardium-myocardium interactions (Figure 57). I then tested the Mtz treatment between 96 hpf and 6 dpf (hereafter referred to as “MTZ 4-6dpf”) (Figure 68A) and I confirmed the successful epicardial ablation (Figure 68B, C).

A

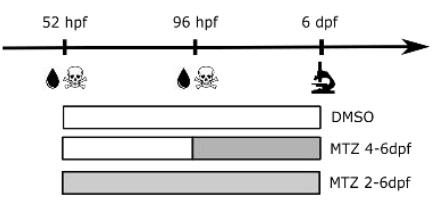

D

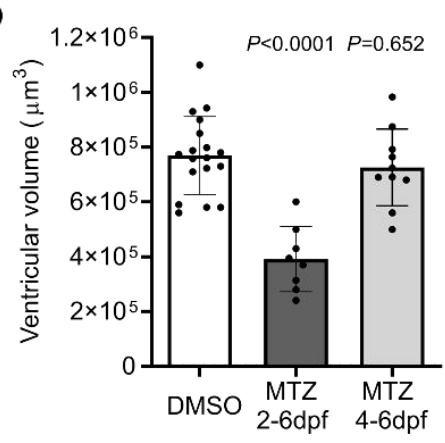

$\mathbf{E}$

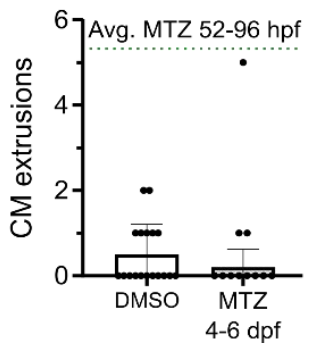

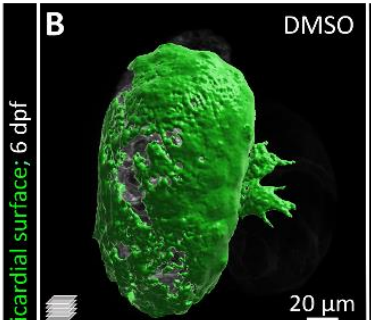
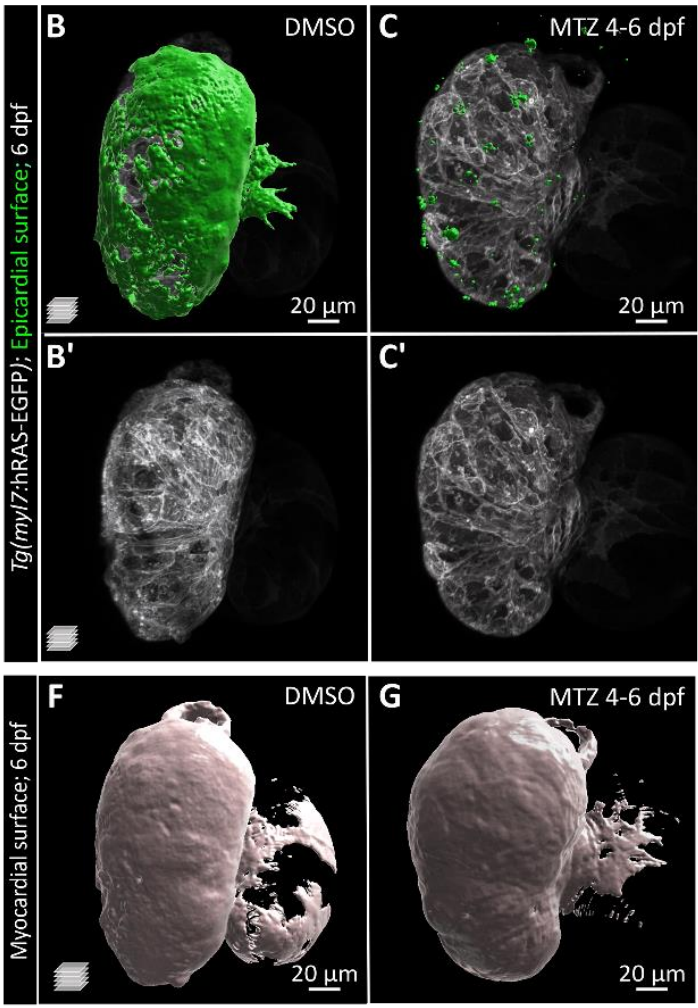

Figure 68: Epicardial ablation after $4 \mathrm{dpf}$ does not affect heart morphology

A) Schematic of the Mtz-treatment setting used for this experiment. B-C') Confocal images of $6 \mathrm{dpf}$ $\operatorname{Tg}(m y)$ l7:bRAS-EGFP);TgBAC(tcf21:mCherry-NTR) DMSO and Mtz-treated larvae. Green, 3D reconstruction of epicardial coverage, showing the lack of epicardium in 4-6 dpf Mtz-treated larvae. D-E) Quantification of ventricular volume (D) and CM extrusions (E) at 6 dpf. The quantification of CM extrusions is compared with the average observed with the $52-96 \mathrm{hpf}$ Mtz treatment. F, G) Reconstruction of the myocardial surface of the hearts shown in $\mathrm{B}, \mathrm{C}$, exhibiting no evidence of $\mathrm{CM}$ extrusion.

To my surprise, I identified no morphological defects in the ventricular morphology and volume ( $P=0.652$ compared with DMSO-treated larvae) (Figure 68B-D). The number of CM extrusions, $0.2 \pm 0.4$, was also comparable with DMSO-treated larvae (Figure 68E-G). These results suggest that the crucial events for the pre-EMT intercellular crosstalk happen during late embryonic and early larval stages (2-4 dpf). After this time-window, the myocardium might be self-sufficient in continuing its growth until $6 \mathrm{dpf}$, likely processing the signaling information received in the previous developmental stages. This tool could be further developed in future studies to identify temporal windows for the onset of EMT and EPDC formation in juvenile stages, which have not yet been described in zebrafish. 


\subsubsection{The presence of the epicardial mechanical barrier is not sufficient to support cardiac growth}

The epicardium is the outermost layer covering the heart and it has been often described as a barrier for the naked myocardium from the external environment (Simoes and Riley, 2018). To distinguish between its mechanical support to the myocardium and its role as a signaling center, I first analyzed if its mechanical role was sufficient to support cardiac growth. To do so, I took advantage of the population of $t c f 21^{1-}$ larvae displaying a number of epicardial cells similar to 96 hpf wild-type hearts (Figure 69A, D).

A
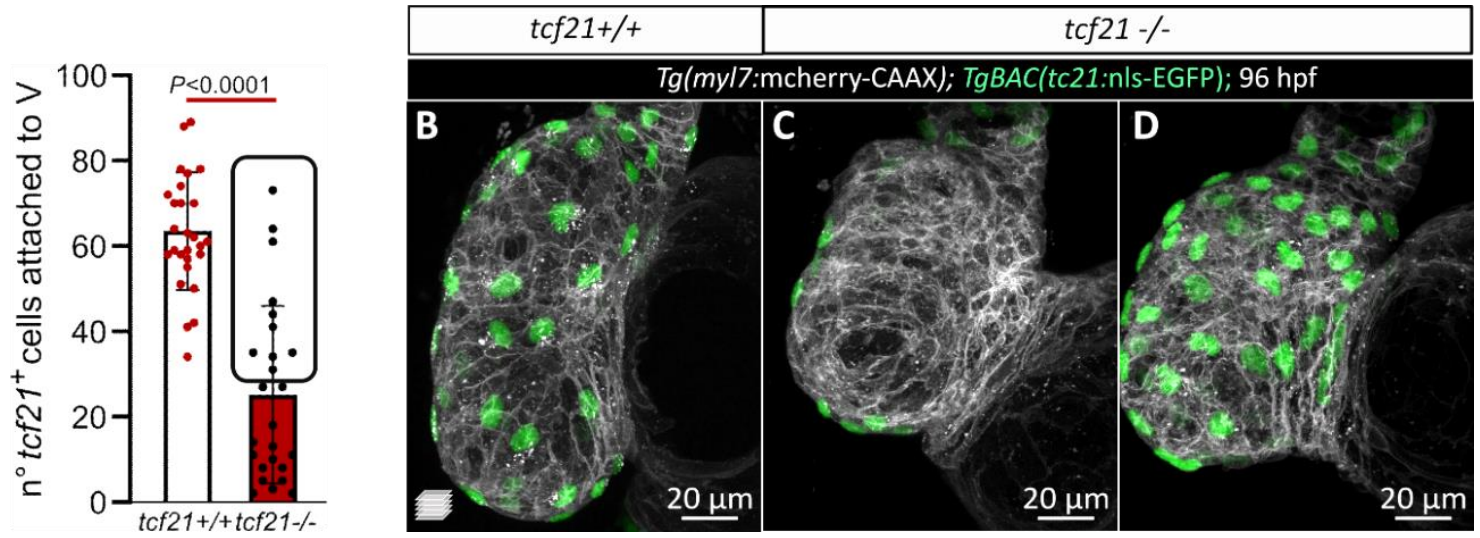

Figure 69: tcf21 mutants exhibit variability in epicardial coverage but not in the ventricular morphology

A) Quantification of ventricular $t c f 21^{+}$cells in $t c f 21^{+/+}$and $t c f 21 \%$ hearts, as shown in Figure 55C, highlighting the population of tc 21 mutants with a wild-type-like epicardial coverage (black box). B-D) Confocal images of $96 \mathrm{hpf} t \mathrm{tc} 21^{+/+}$and $t c f 21 /$ hearts. Both the categories of mutants (C, D) exhibit a smaller ventricle compared to wild types (B), independent of their epicardial coverage.

I first compared the heart morphology of these larvae (Figure 69D) with tcf21 mutants devoid of epicardial coverage (Figure 69C), and I observed that the two categories of mutants were indistinguishable from each other. I also noticed that several extruding CMs were covered by epicardial cells, indicating that the sole epicardial physical barrier is not sufficient to prevent the extrusions (Figure 70).

\section{$\operatorname{Tg}(m y / 7: B P F-C A A X) ; \operatorname{TgBAC}(t c f 21: \mathrm{nls}-\mathrm{EGFP}) ; 76 \mathrm{hpf}$}

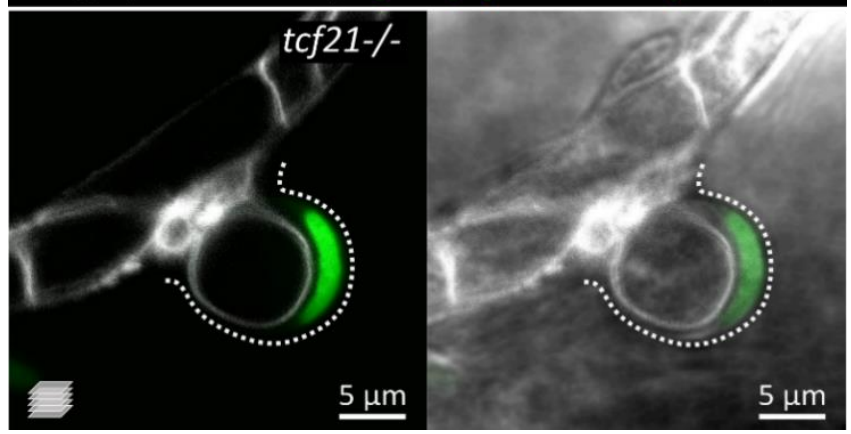

Figure 70: CM extrusion are occasionally covered by epicardium Confocal images of a $76 \mathrm{hpf} t \mathrm{cf} 21 \%$ heart, exhibiting a CM extrusion (white, CM membrane) covered by a $t c f 21^{+}$epicardial cell (nucleus, green; cell body highlighted with dashed line). 
With the aim of quantifying possible differences in the cardiac phenotype among these mutants depending on their epicardial coverage, I measured the correlation between the number of ventricular $\operatorname{tcf} 21^{+}$cells and different mutant defects. Notably, I found no significant correlation between the number of $\operatorname{tc} 21^{+}$cells attached to the myocardium and the ventricular volume (Figure 71A), or the number of extruding CMs (Figure 71B). Altogether, these observations suggests that the epicardium fuctions not only as a mechanical barrier protecting the ventricle. In order to promote cardiac morphogenesis, the epicardium must play a role as a reservoir of molecules, either contributing to the extracellular environment or directly signaling to CMs. Based on these results altogether, I speculate that the epicardium provides growth factors to the myocardium, particularly between 2 and $4 \mathrm{dpf}$, which in turn activate signaling programs instructing CMs to continue their growth.
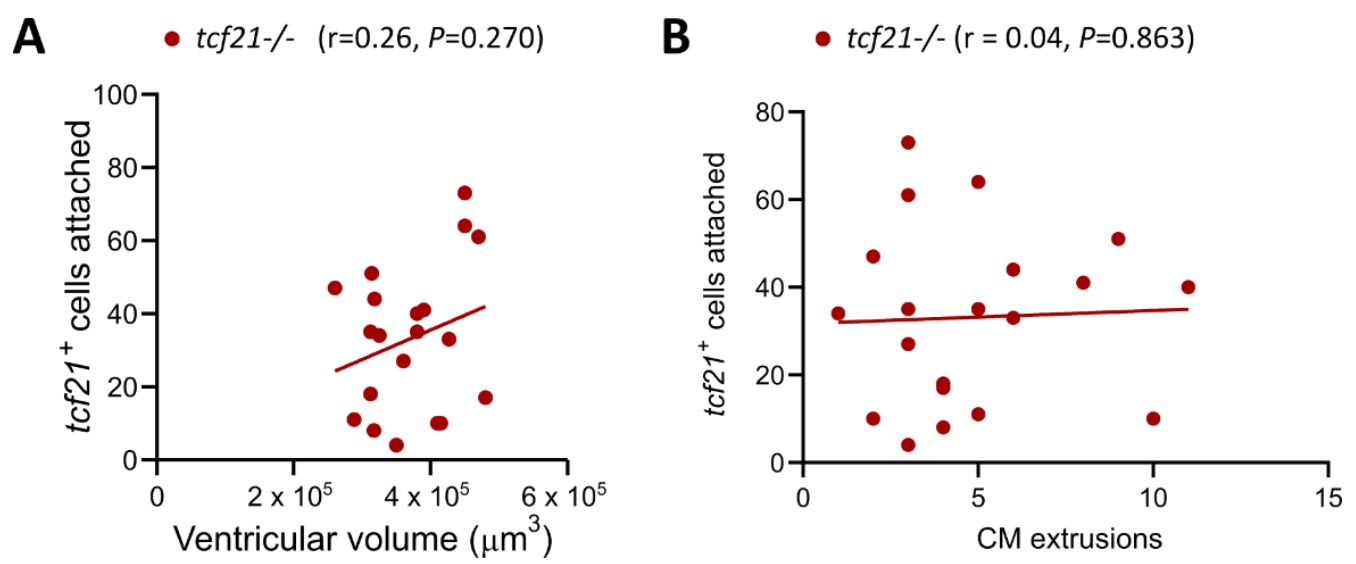

Figure 71: The ventricular defects in $t c f 21^{/-}$larvae does not depend on the number of $t c f 21^{+}$cells A) Pearson correlation between ventricular volume ( $\mathrm{X}$ axis) and number of ventricular $t c 21^{+}$cells ( $\mathrm{Y}$ axis) in $96 \operatorname{tcf} 21 \%$ larvae. B) Pearson correlation between number of CM extrusions ( $\mathrm{X}$ axis) and number of ventricular $t c f 21^{+}$cells ( $\mathrm{Y}$ axis) in $96 \operatorname{tcf} 21 \%$ larvae.

\subsubsection{The epicardium serves as a reservoir of signaling molecules necessary for myocardial development}

After observing the need for the epicardium not only as a physical barrier, I aimed to find important signals regulating the crosstalk with the myocardium. Therefore, I took advantage of the tcf21 mutants exhibiting good epicardial coverage (Figure 69D) to identify missing epicardial factors necessary for proper myocardial growth.

I manually extracted $96 \mathrm{hpf} \operatorname{TgB} A C(t c f 21: n l s-E G F P) ;(m y l 7: m C h e r r y-C A A X)$ wild-type and tcf21 mutant hearts (Figure 72A). I dissociated them in single-cell suspensions and performed fluorescent-activated cell sorting (FACS). With FACS, I could select only live and intact cells (DAPI) and sort different cellular populations. 
A

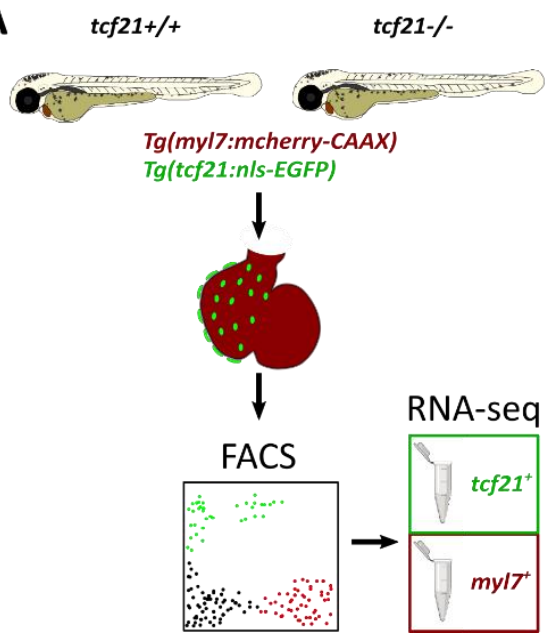

B

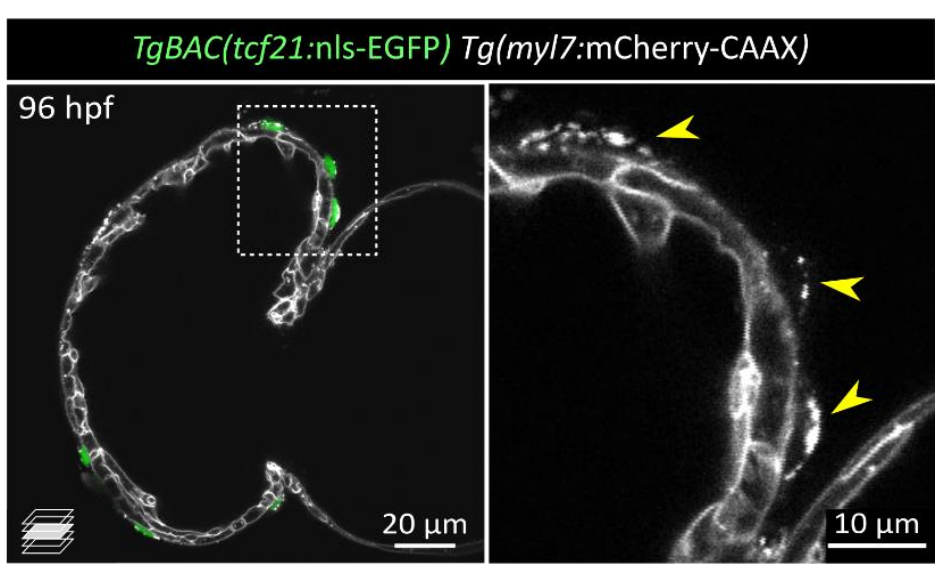

Figure 72: Schematic of the RNA-seq

A) Schematic depicting the setting used for the RNA-seq on $t c f 21^{+}$and $m y l 7^{+}$cells, comparing $t c f 21^{+/+}$and tcf $21 \%$ hearts. B) Confocal images showing $\mathrm{Tg}(\mathrm{my} / 7$ :mCherry-CAAX) dotted expression in $96 \mathrm{hpf}$ epicardial cells (arrowheads point to $\operatorname{tcf} 21^{+}$cells).

It was essential to only select hearts which were positive for both the transgenes, since I noticed that many epicardial cells also expressed $\mathrm{Tg}$ (myl7:mCherry-CAAX) (Figure 72B). It would be interesting in future studies to investigate the biological relevance of this unexpected expression, to understand if it is restricted to the exogenous transgene or also affecting the endogenous

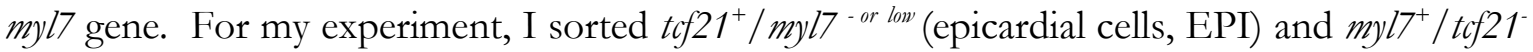
(CMs) cells in separate tubes (Figure 73) from both wild-type and mutant samples. We then performed transcriptomic analyses on all four samples (CM W'T, CM mutants; EPI WT, EPI mutants).

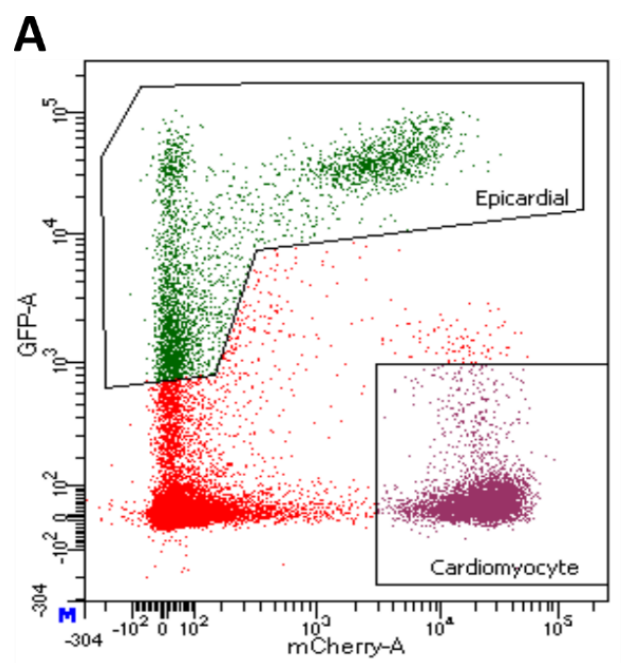

\section{B}

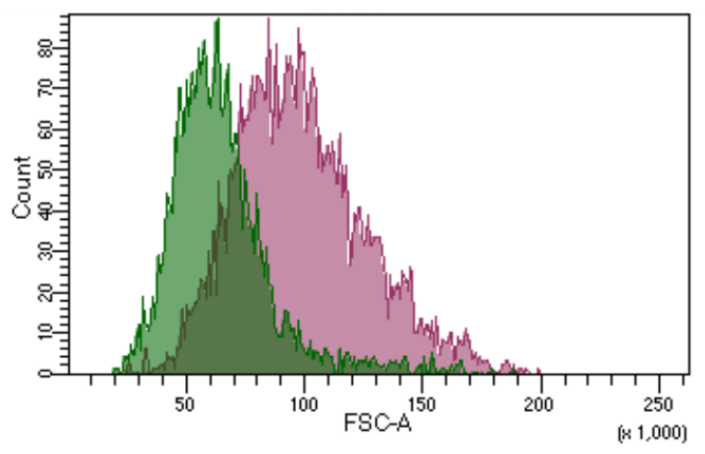

Figure 73: FACS sorting of epicardial cells and CMs

A) FACS graph depicting the gating selected to sort epicardial cells (green; $\mathrm{GFP}^{+}$) and $\mathrm{CMs}$ (purple; $\mathrm{mCherry}^{+}$, GFP-). The population of epicardial cells showing myl7:mCherry-CAAX expression described in Figure 72B is represented by the asterisk. B) The two populations exhibited a clear distinction in the forward scatter (FSC-A; X-axis), representing the difference in cell size between these cell types. 
Notably, this dataset also represents an important resource to identify genes preferentially expressed in wild-type CMs or epicardial cells. As a preliminary approach, I ran Gene Ontology (Ashburner et al., 2000; Gene Ontology, 2021)(Figure 74A) and Reactome (Fabregat et al., 2018; Jassal et al., 2020)(Figure 74B) analyses on EPI or CM enriched genes to identify biological and molecular processes specific to either cell type. Altogether, I observed that the $\mathrm{CM}$ most enriched terms comprised metabolism, mitochondrial processes, respiration, and striated muscle development. Conversely, among the EPI-enriched terms, I could identify the large categories of epithelial migration/chemotaxis, cell-cell communication, growth factors signaling, and extracellular matrix organization (Figure 74).

A

\section{Biological Processes}

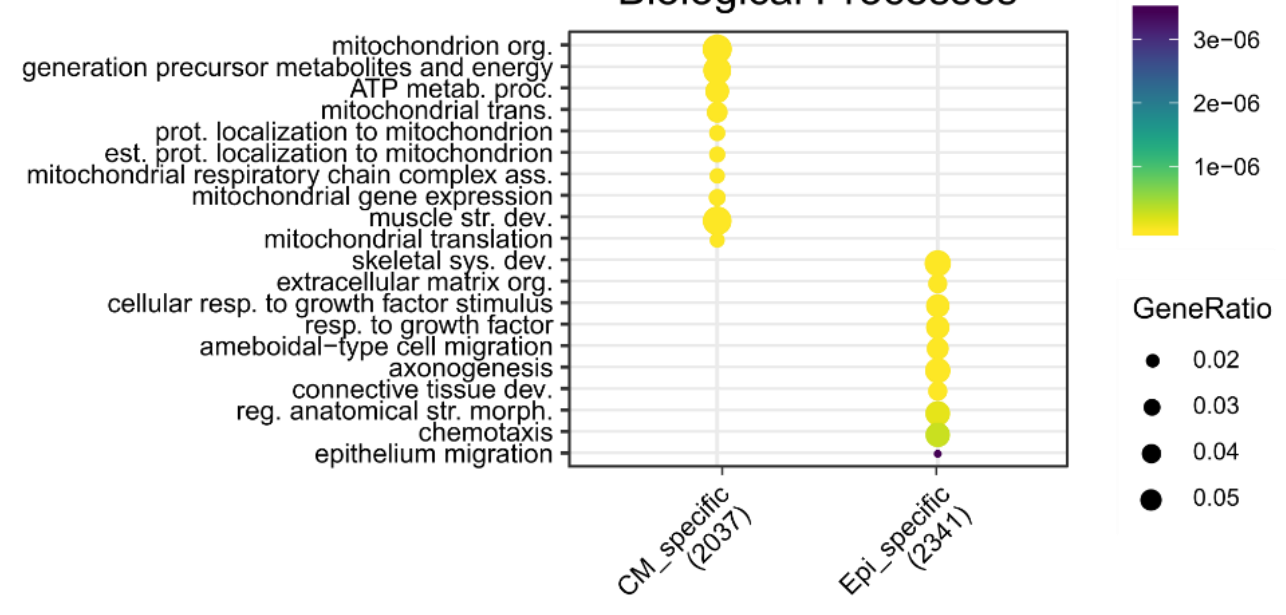

B

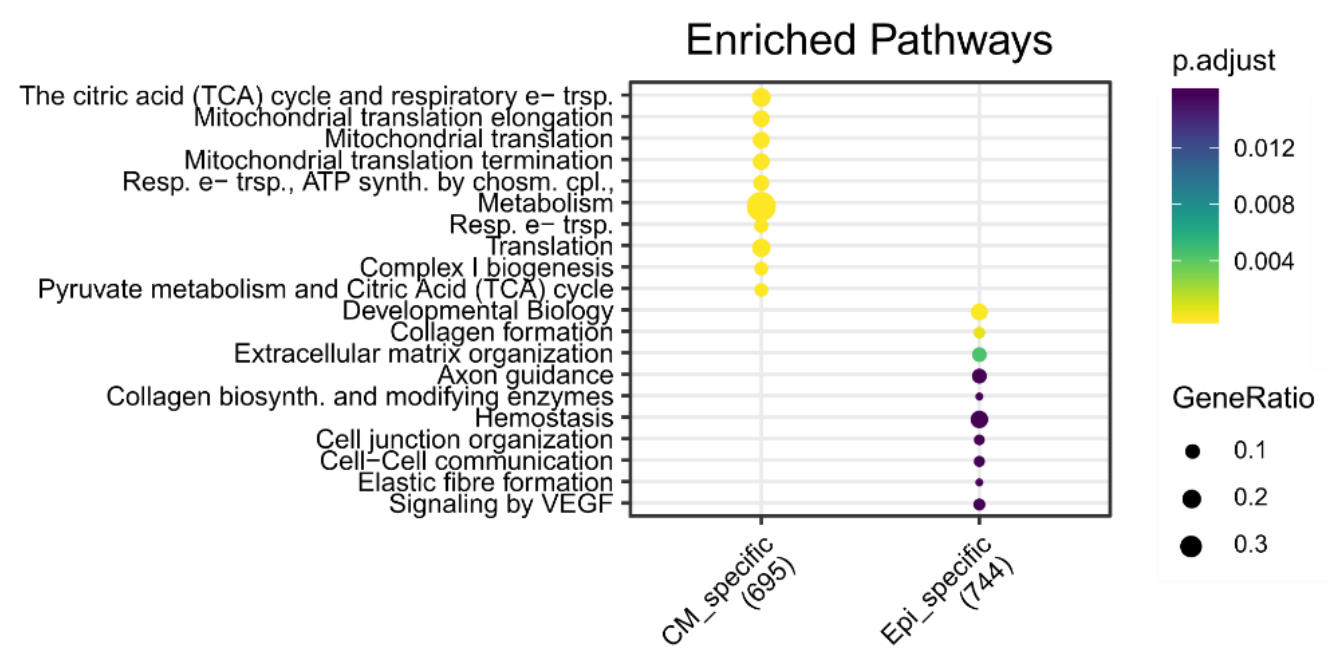

Figure 74: CMs and epicardial cells display differential gene expression

A, B) Bubble plots showing the most overrepresented Gene ontology (A) and Reactome (B) categories in CM- or EPI- differentially expressed genes. Both the analyses show an enrichment of metabolism/mitochondrial processes-related terms in CMs and migration/cell-communication/ECMrelated terms in epicardial cells. 
Next, I aimed to understand which secreted factors are expressed specifically by epicardial cells, and could mediate the epicardium-myocardium crosstalk in the zebrafish heart. To identify those genes, I cross-referenced the EPI- and CM-enriched genes lists with the zebrafish Matrisome (Nauroy et al., 2018) "ECM structural components" and "secreted factors" (Figure 75A). With this approach, I identified 56 secreted factors genes and 174 ECM component genes specifically expressed (or significantly enriched) in epicardial cells, consistent with the prominent secretory activity of these cells (Y. Li et al., 2017). Conversely, among the CM enriched genes, only 20 were secreted factors genes and 31 were ECM genes. Among the extracellular secreted component genes enriched in the epicardium (Figure 75B), several components have been previously reported to be crucial for adult epicardium-myocardium crosstalk (Perez-Pomares et al., 2002; Olivey and Svensson, 2010; Y. Li et al., 2017), including FGF, TGF- $\beta$, IGF, and PDGF family members. These results provide further confidence that our dataset reflects the molecular processes involved in this intercellular communication.

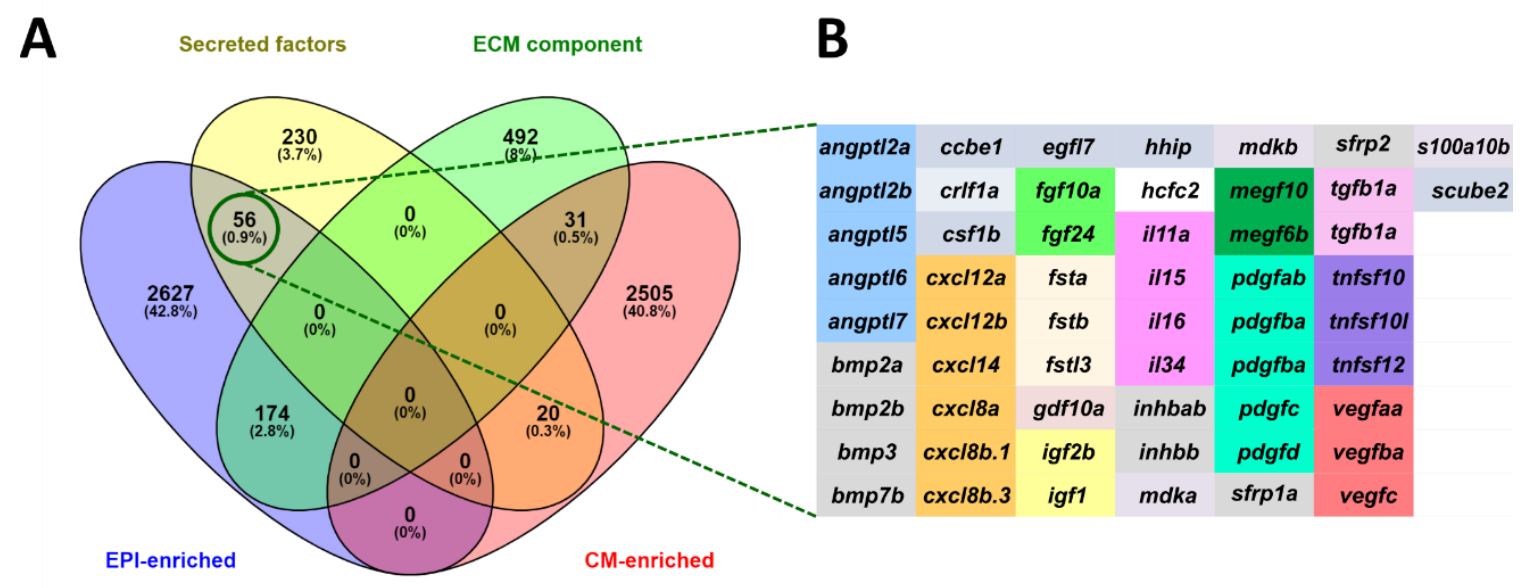

Figure 75: The expression of several secreted factor genes is enriched in the epicardium

A) Venn diagram obtained from Venny (J. C. Oliveros, 2007-20015), comparing epicardial (EPI)- or CMenriched genes with different categories of genes listed in the zebrafish matrisome (Nauroy et al., 2018). B) List of the 56 secreted factor genes enriched in the epicardium. The different colors in the table are used to highlight genes belonging to the same gene family.

Then, I aimed at identifying epicardial secreted factors, and possibly myocardial-expressed binding partners, downregulated in $\operatorname{tcf} 21^{1 /}$ hearts. Various secreted factors were represented among the downregulated genes in $t c f 21^{1}$ epicardial cells: csf1b, fgf 24 , vegfaa, $c x c l 14$, angptl6, tdgf1, $n t f 3$, igfbp5b (Figure 76). When looking for potential partners of these ligands in the myocardium, I identified particularly interesting receptors for $c s f 1 b, f g f 24, c x c l 14: c s f r 1 a, f g f r 4$, and cxcr3.1, respectively (Figure 76). 


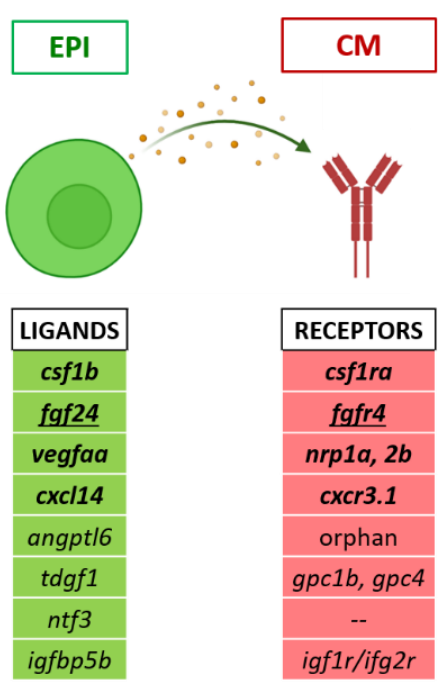

Figure 76: Secreted factor genes downregulated in $t c f 21 /-$ epicardium and their $\mathrm{CM}$ receptors List of secreted factor genes downregulated in the $t c f 21 \%$ epicardium (green) and their receptor genes expressed in $\mathrm{CMs}$ (red). Ntf3 is not known to bind any CM-expressed receptor. Angptl6 is an orphan ligand, as all the other Angptl proteins.

Other ligands, such as Vegfaa, do not have a known receptor prominently expressed in CMs, while kdrl (vegfr2) is 97 fold-change enriched in the epicardium. Therefore, I speculate that some of these ligands could signal in an autocrine manner to epicardial cells, regulating the expression and potentially the secretion of other important molecules from this tissue.

\subsubsection{Vegf and Fgf signaling are crucial in the epicardium-myocardium crosstalk during zebrafish heart development}

Among the identified factor genes, fof 24 and vegfa $a$ were of particular interest. On one hand, the FGF pathway is well known for mediating epicardium-myocardium crosstalk, while the available literature mostly focused on its mitogenic role on CMs (Pennisi et al., 2003; Lavine et al., 2005). On the other hand, Vegfa is a well-known player in angiogenesis and, during heart development, it has been implicated in coronaries formation (B. Wu et al., 2012; Karra et al., 2018), a process partially involving epicardial cells. Nonetheless, there is no consensus regarding its cardiac expression pattern (especially in mouse) (Tomanek et al., 1999; Lavine et al., 2006; B. Wu et al., 2012; Karra et al., 2018), and its role in the developing epicardium has so far not received much attention.

Thus, I first attempted to clarify the zebrafish expression pattern of vegfaa during the early larval stages. I could infer epicardial-enriched expression of vegfaa from the transcriptomic analysis, having a 10 times higher expression in EPI than in CMs. Moreover, using the previously described TgBAC(vegfaa:EGFP) transgenic line (Karra et al., 2018), I could only observe GFP expression in the epicardium at both 76 and $96 \mathrm{hpf}$, colocalizing with $\operatorname{Tg}(\operatorname{tcf} 21: m C h e r r y-N T R)^{+}$ cells (Figure 77A, B). In addition, while vegfaa:EGFP expression was completely absent from 
CMs or endocardial cells at the analyzed stages, I observed it in 96 hpf SMCs (Figure 77B), some of which are known to derive from an epicardial cluster (Weinberger et al., 2020). Unfortunately, I could not assess the expression pattern of fof 24 or its receptor fof 4 due to the lack of transgenic lines available and the limited resolution of ISH and FISH at these larval stages. However, from the transcriptomic data, fof 24 displayed a 27 fold enrichment in epicardial cells, close to values obtained for genes as $\operatorname{tcf} 21$ (31 fold enrichment). Moreover, the mouse ortholog of $f g f 24, \mathrm{Fg} f 18$, also exhibited restricted expression in the epicardium and valve interstitial cells (Hagan et al., 2019).

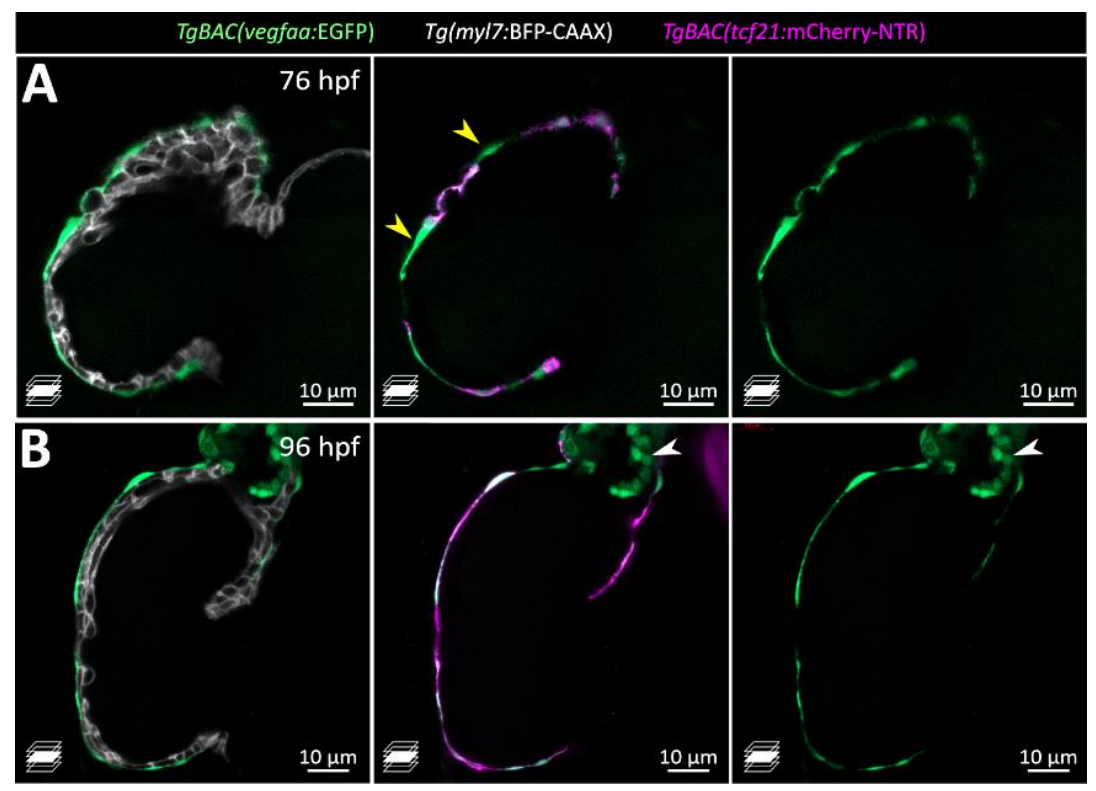

Figure 77: $T g B A C$ (vegfaa:EGFP) larval cardiac expression is restricted to the epicardium and SMCs A-B) Confocal images of 76 (A) and 96 (B) hpf TgBAC(vegfaa:EGFP);(myl7:BFP-CAAX);(tcf21:mCherry-NTR) ventricles. TgBAC(vegfaa:EGFP) expression is restricted to the epicardium and OFT SMCs (B, white arrowheads). Yellow arrowheads point to vegfaa:EGFP ${ }^{+}$but $t c f 1$ : $m$ Cherry epicardial cells.

Remarkably, both FGF and VEGF share a common intracellular signal transduction, the mitogen-activated protein kinase (MAPK)/ extracellular regulated kinase (ERK) pathway.

Therefore, to characterize whether these downstream effectors were affected by the loss of the epicardium, I first aimed at analyzing the ERK signaling in CMs following epicardial ablation. I took advantage of a transgenic line recently generated in our lab, Tg (myl7:ERK-KTR-Clover-tag H2B-mScarlet), that serves as a biosensor of the ERK phosphorylation, by using a kinase translocation reporter (KTR). KTRs, fused to a fluorescent protein (i.e., Clover in this case), are kinase substrates containing phosphorylation-sensitive nuclear localization (NLS) and export (NES) signals (Kudo et al., 2018). Therefore, once the ERK kinase is active, KTR is phosphorylated and exported into the cytoplasm, leading to cytoplasmic Clover localization (de la Cova et al., 2017). To monitor the ERK kinase activity following epicardial ablation, I assessed the percentage of inactive ERK (nuclear-Clover) CMs at 80 and $100 \mathrm{hpf}$ (Figure 78A). 
From preliminary experiments, the percentage of CMs with inactive ERK signaling was significantly enriched in hearts devoid of epicardium (Figure 78B). Interestingly, the downregulation of the pathway precedes the morphological phenotype (appearing at $96 \mathrm{hpf}$ ). These results suggest that the epicardial-derived downregulation of ERK might be one of the underlying causes of the observed phenotype. Nonetheless, further future experiments are needed to substantiate this hypothesis.
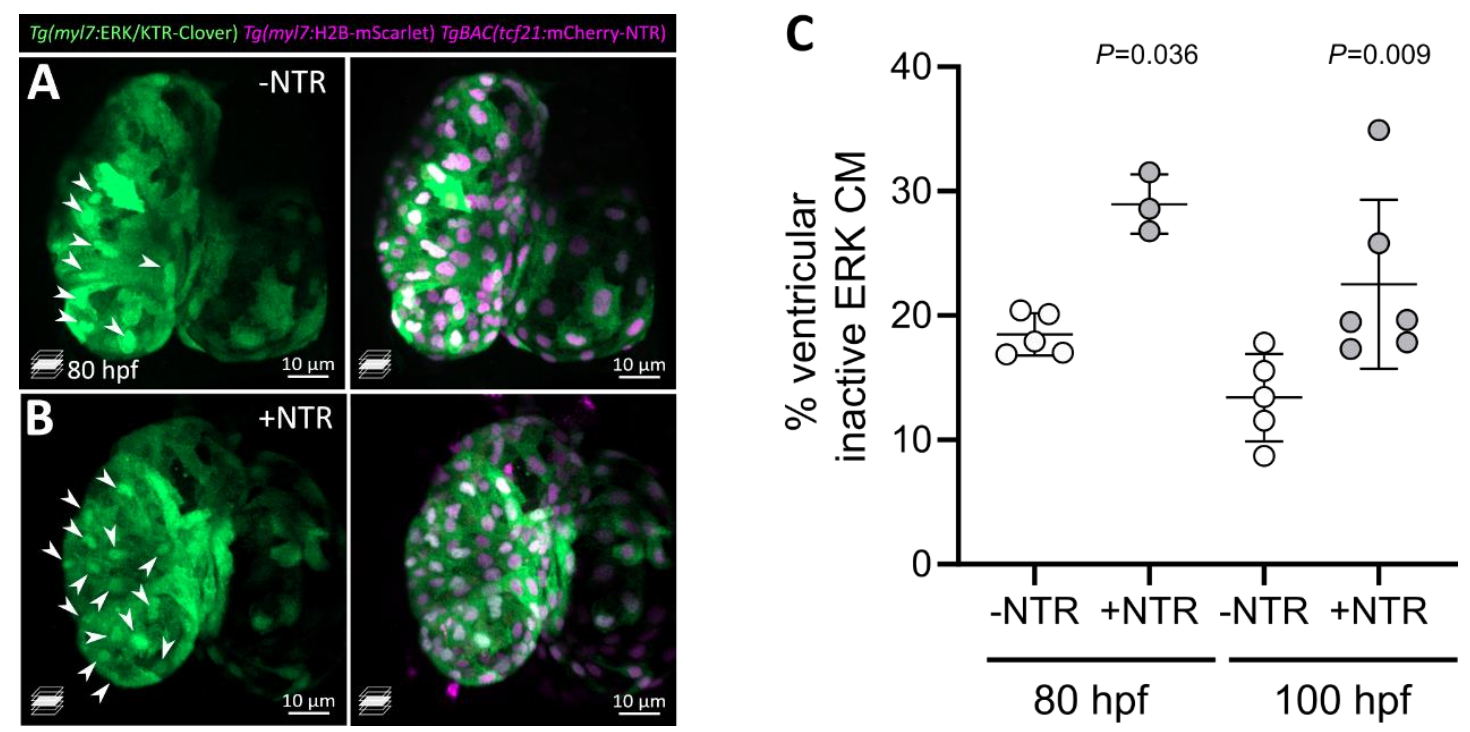

Figure 78: The ERK signaling pathway is downregulated in CMs following epicardial ablation A-B) Confocal images of 80 (A) and 96 (B) hpf $\mathrm{Tg}$ (myl7:ERK-KTR-Clover-tag H2B-mScarlet) ventricles in control (-NTR) and TgBAC(tcf21:mCherry-NTR) (+NTR) larvae treated with MTZ. White arrowheads point to nuclear Clover (inactive ERK) CMs. C) Quantification of the percentage of inactive ERK (nuclear Clover) ventricular CMs in control (-NTR) and TgBAC(ttf21:mCherry-NTR) (+NTR) larvae treated with MTZ.

To better understand the involvement of VEGF and FGF signaling pathways in the myocardium at the early larval stages, I took advantage of small molecule inhibitors. Thus, I used SU5402 (FGFR inhibitor) and SU5416 (VEGFR inhibitor) to block the downstream signaling from 65 to $96 \mathrm{hpf}$. To avoid complicating the interpretation of the results, I decided to treat the larvae after $60 \mathrm{hpf}$. It was recently reported a high rate of proliferation in compact layer CMs between 48 and $60 \mathrm{hpf}$, which is also regulated by the MAPK pathway (Priya et al., 2020). Notably, in larvae treated with as little as $0.75 \mu \mathrm{M}$ VEGFR inhibitor and $1 \mu \mathrm{M}$ FGFR inhibitor, I observed a reduction of the ventricular size compared with DMSO-treated larvae (Figure 79A-C). When looking at CM morphology, the inhibitor-treated hearts displayed a

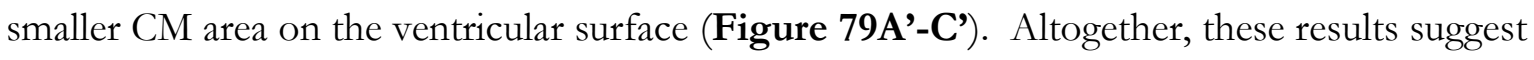
an involvement for epicardial FGF and VEGF signaling through the MAPK/ERK downstream CM pathway, in modulating ventricular morphogenesis. Further experiments will be required to investigate the ligand-receptor interactions involved in this signaling and their molecular role in balancing hyperplastic vs hypertrophic CM growth. 

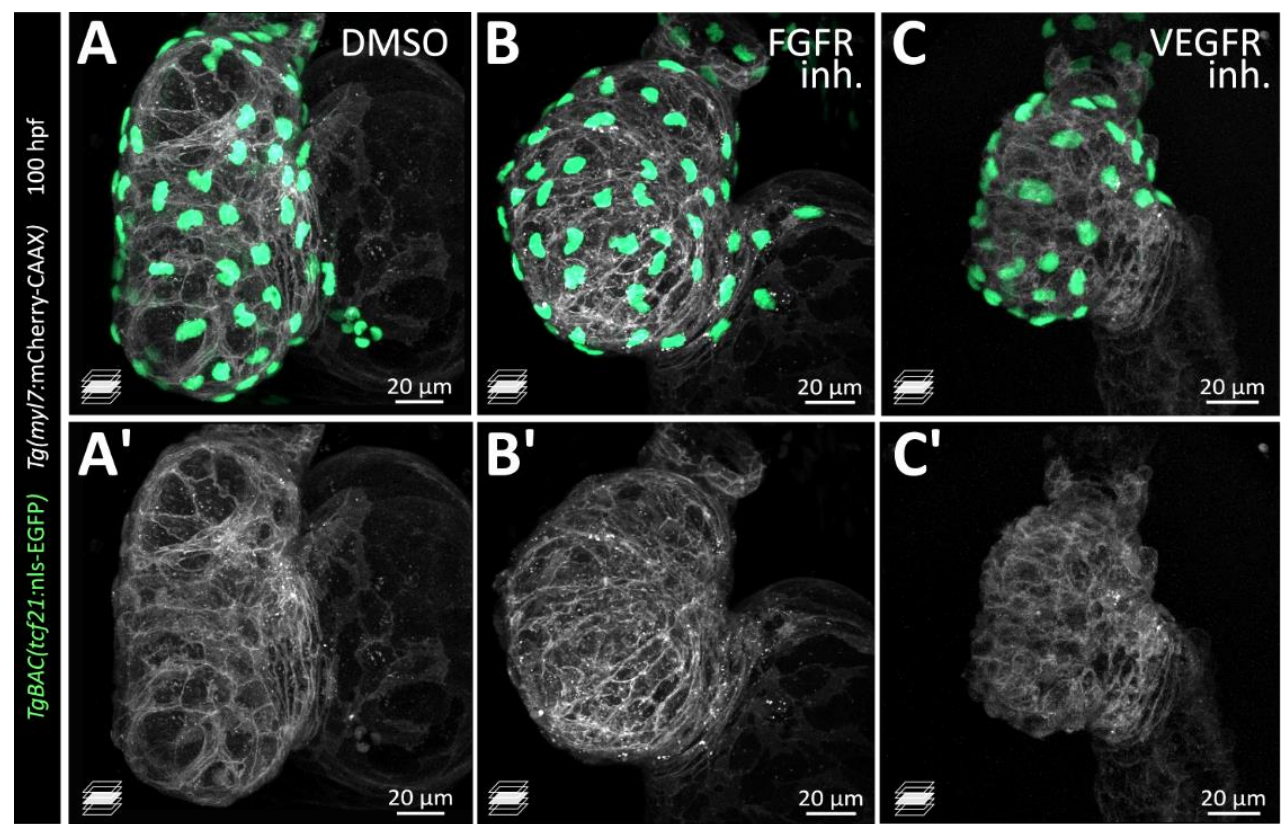

Figure 79: FGF and VEGF pathway inhibitions reduce ventricular size in wild-type larvae

A-C') Confocal images of $100 \mathrm{hpf} \operatorname{TgB} A C(\operatorname{tc} 21: n l s-E G F P) ;(m y l 7: m C h e r r y-C A A X)$ ventricles in treated with DMSO (A), SU5402 (FGFR inh., B), or SU5416 (VEGFR inh., C). A', B', C' show only the myocardial surface composed of smaller looking CMs.

\subsubsection{Proposed model}

In this second work, I propose a previously unappreciated role for the epicardium in driving ventricular morphogenesis.

1) I established three partially independent models of impaired epicardial coverage during zebrafish development. The refinement of the Mtz ablation in larval stages will be useful for future studies, allowing one to investigate the effects of epicardial loss in specific time-windows.

2) The loss of epicardium or epicardial function compromises CM volume increase between 3 and $4 \mathrm{dpf}$, without affecting CM number or proliferation. The dependency of these defects on the epicardial loss was confirmed by their rescue following epicardial regeneration.

3) The ablation of the epicardium at late larval stages (4-6 dpf) does not affect ventricular growth or integrity, suggesting that the time window 52-96 hpf is crucial for epicardium-myocardium interactions during development.

4) I generated a dataset to analyze epicardial-specific secreted factors important to drive epicardium-myocardium crosstalk and identified defective epicardial-derived FGF and VEGF signaling in tcf 21 mutants. I further propose that the downregulation of the common downstream ERK pathway in CMs might be partially responsible for the ventricular defects. Further experiments would shed light on the precise involvement of these pathways in different processes regulating $\mathrm{CM}$ growth. 
Results 


\section{Discussion}

\subsection{The endothelium-smooth muscle crosstalk in the developing cardiac outflow tract is orchestrated by TGF- $\beta$ /Alk5 signaling}

Many different cell types during embryogenesis converge and communicate to give rise to the cardiac OFT (Neeb et al., 2013; Fountoulaki et al., 2015). Its complex origin and intricate signaling make this structure a focal point of multiple congenital diseases (Neeb et al., 2013). Nonetheless, these cellular and signaling interactions have not been fully elucidated. In this thesis, I show that the TGF- $\beta$ signaling pathway plays a pivotal role in OFT ECs, from which it modulates the extracellular environment. The ECM, in turn, affects EC and SMC proliferation, migration, and organization, orchestrating the development of the OFT.

\subsubsection{The development of the OFT, a hub for intercellular interactions}

Most of the literature focusing on heart development has thoroughly described atrial and ventricular development, while the formation of the OFT still requires further investigation, particularly in zebrafish. Here, I present experimental evidence on the formation of the OFT and the VA in the zebrafish embryo and their cellular composition, which complements and expands two recent studies (Duchemin et al., 2019; P. Sidhwani et al., 2020). By high-resolution live imaging, I observed that the OFT forms at $24 \mathrm{hpf}$ initially as a constriction of the heart tube located at the arterial pole of the ventricle. At this time, the OFT is composed of ECs, CMs, and undifferentiated mesodermal cells, the latter occupying the most rostral location. I subsequently observed an increase in OFT size between 36 and $72 \mathrm{hpf}$, particularly affecting the number of ECs. The cause of this growth has been identified in the accrual of ECs by proliferation and addition from adjacent vessels (Pragya Sidhwani et al., 2019), as well as in the appearance of SMCs (Grimes et al., 2006; Felker et al., 2018). Notably, up to 72 hpf, I observed that SMCs are not yet mature and terminally differentiated. Indeed, these cells lack the expression of typical SMC mature markers, such as Acta2 and Tagln, and actin bundles (as confirmed by TEM imaging). It will be important to further characterize the maturation of OFT SMCs, as well as the precursors from which they originate. It is known that, despite previous beliefs, OFT SMCs do not seem to derive from transdifferentiated CMs (Grimes et al., 2006; Grimes et al., 2010), while they originate from SHF precursors (Zhou et al., 2011). However, whether these cells are progressively added to the OFT already in a partially differentiated state, or they derive from the undifferentiated mesodermal cells present in the OFT at $30 \mathrm{hpf}$, is still to be determined.

The OFT is connected to the vast vascular network through the VA, the ortholog of the mammalian aorta, but its development in zebrafish is largely unknown. Here, by 


\section{Discussion}

photoconversion experiments associated with time-lapse imaging, I identified a distal population of OFT ECs, which contributes to the VA formation by being displaced in the direction of the blood flow. These results partially differ from previous studies, which describe the movement of ECs towards the heart from the aortic arches at earlier time points (Rochon et al., 2016). It is appealing to think that the EC deriving from the aortic arches, which first move into the distal OFT, are the same cells later giving rise to the VA. It is also conceivable, based on the study from Sidhwani et al. (P. Sidhwani et al., 2020), that EC proliferation and displacement could involve different sets of ECs in the OFT. For instance, the proximal population of ECs, which from my experiments appears more proliferative and does not contribute to the VA, could be responsible for the increase in the size of the OFT. The study of the relationship between EC movements and proliferation will require high-resolution live imaging coupled with photoconversion in a longer time window. Together, it will be important to uncouple the genetic and mechanical processes involved in EC proliferation and migration, taking advantage of the OFT and VA to shed light on these two fundamental endothelial processes of broader relevance.

\subsubsection{The role of Alk5/ TGF- $\beta$ in the OFT is cell-type- and time-dependent}

The TGF- $\beta$ pathway controls several cellular processes in development and disease, such as cell proliferation, adhesion, migration, and differentiation. However, the multi-functional role of the pathway prompts several context- and tissue-dependent activities difficult to disentangle. Moreover, most of the studies have been performed in vitro, often leading to opposing results (e.g.,(Seay et al., 2005; Suwanabol et al., 2012), likely due to factors as cellular density, cell origin, medium composition, and the surrounding matrix. Therefore, it is imperative to study the TGF- $\beta$ signaling pathway in an in vivo setting.

Here, I present a model of Alk5/TGF- $\beta$ signaling impairment during zebrafish development. I found that, up to $72 \mathrm{hpf}$, the only observable morphological phenotype affects the cardiac OFT, while the expression of alk 5 was observed in the whole heart. Notably, I observed a refined activation of the TGF- $\beta$ pathway in OFT ECs, which could explain the specificity of the defects. Many different levels of regulation are in place to prevent premature and unrequired activation of the pathway in particular tissues or developmental stages (Feng and Derynck, 2005; Derynck and Budi, 2019). To name a few possibilities, these regulatory mechanisms can: control the release of the TGF- $\beta$ ligands from the ECM; inhibit the activation of the receptor by binding to its intracellular domain (i.e., FKBP12 and Smad7); regulate the expression of abundance of the Smad proteins; regulate the binding of Smads to DNA (e.g., Ski proteins) (Stroschein et al., 1999; Tecalco-Cruz et al., 2018; Yan et al., 2018). 
Importantly, I identified an earlier effect of the loss of Alk5 on ECs, and a later one on SMCs. The role of Alk5 in ECs has been extensively studied only in vitro, where it promotes EC quiescence (Goumans et al., 2002; Goumans, Valdimarsdottir, et al., 2003; Maring et al., 2016). In our zebrafish model, I confirmed that the pivotal role of Alk 5 in restricting EC proliferation is conserved in vivo and it is relevant for cardiovascular development. Interestingly, while Alk5 restricts the proliferation of some OFT ECs, it promotes the displacement of others towards the VA, indicating a very cell-specific role even within the same cell type. Moreover, at later stages $(72-80 \mathrm{hpf})$, Alk5 regulates SMC organization and behavior, coincidental with the activation of the TGF- $\beta$ pathway in these cells. I hypothesize that timings of activation of the pathway are key in explaining the defects caused by its impaired function. For instance, in mice, only the EC-specific Alk.5 KO (Carvalho et al., 2007; Sridurongrit et al., 2008) recapitulates the global KO phenotype (Larsson et al., 2001; Seki et al., 2006). This result is in contrast with its expression, which appears to be restricted to the SMC wall of the aorta at E11.5 (Seki et al., 2006). Nonetheless, the reported SMC expression coincides with a stage when the defect of Alk. $5^{-1}$ embryos is already extremely severe (most Alk.5\% embryos die at E10.5). These discrepancies could be explained by a very dynamic expression of Alk.5 or the TGF- $\beta$ pathway activation in different cell types, which will need further investigation. The specific dissection of the regulatory events, as well as the deeper analysis of tissue-specific mutants for different pathway components, will help to elucidate the different cell-specific requirements of the TGF$\beta$ pathway. (Certain lines in this subsection have been quoted verbatim from Boezio et al., eLife 2020;9:e57603 for the scientific accuracy of the terms.)

\subsubsection{ECs regulate SMC behavior by modulating the ECM environment}

Unexpectedly, I found that overexpressing alk.5 wild-type form exclusively in ECs largely restored OFT morphology and function of alk.5 mutants. Notably, alk.5 overexpression in ECs was sufficient to restore the SMC wall, suggesting a crucial crosstalk between these two cell types. Recently, it was described that pericytes-specific deletion of Alk.5 in mice caused an enlargement of brain capillaries due to an EC hyperproliferation state, which was mediated by impaired ECM degradation (Dave et al., 2018). Moreover, previous studies speculated an early role for TGF- $\beta$ in ECs during yolk sac vasculogenesis and a later requirement in vascular SMCs for the complete development of the vascular network. The authors further proposed that the SMC phenotype was a direct consequence of EC impairment (Carvalho et al., 2007). These two studies corroborate our results, suggesting critical crosstalk between ECs and mural cells regulated by Alk5.

It is well known that the individual functions of both ECs and SMCs cells depend on their reciprocal interaction during development and in homeostatic conditions (Gaengel et al., 2009; Fountoulaki et al., 2015; Stratman et al., 2017; M. Li et al., 2018; Perbellini et al., 2018). ECs 


\section{Discussion}

initiate the communication sending signals to recruit mural cells around the vessels (Hellstrom et al., 1999; Lindblom et al., 2003; Ando et al., 2016; Stratman et al., 2017). These cells reciprocate with different cues, which, in turn, affect ECs biology. It has been shown that these interactions occur in a variety of ways, which can require physical contacts, secreted mediators, or the deposition of ECM components (Hirschi et al., 1999; Gaengel et al., 2009; Fountoulaki et al., 2015; Perbellini et al., 2018).

Our transcriptomic profiling identified differential expression of genes encoding ECM and cellto-matrix adhesion genes following manipulation of Alk5 function. The ECM is normally secreted by both ECs and SMCs (Kelleher et al., 2004; G. E. Davis and Senger, 2005). Therefore, I speculated that the ECM would serve as a scaffold for the crosstalk among the different cell-types also in the OFT. In particular, the OFT defects in alk 5 mutants likely derive from different roles of the ECM, both as a signaling mediator and mechanical support (Raines, 2000; Kelleher et al., 2004; G. E. Davis and Senger, 2005). An interesting gene downregulated in Alk5 inhibitor-treated larvae was $f b \ln 5$, encoding an integrin-binding ECM component (Nakamura et al., 1999). Fbln5 is expressed in ECs and fibroblasts/SMCs in mouse (Tabula Muris et al., 2018; X. Liu et al., 2019) and also in zebrafish. Here, I showed that EC-specific of fbln 5 can partially rescue alk 5 defects. Multiple functions have been reported for Fbln5, both in assembling the elastic lamina (Nakamura et al., 2002; Chapman et al., 2010), in restricting SMC-proliferation (Schiemann et al., 2002), and promoting EC-to-matrix adhesion in vitro (Preis et al., 2006). Therefore, Fbln5 could act in different ways to support OFT morphogenesis downstream of Alk5, both by regulating EC attachment to the ECM, thereby restricting their proliferation (Sullivan et al., 2007; Williamson et al., 2007), and in promoting the formation of the OFT elastic wall.

However, our results do not resolve whether ECs are the only cells in which Alk5 is necessary to direct OFT development. It is interesting to note that the SMC-specific deletion of the type II TGF- $\beta$ receptor, Tgfbr2, leads to a similar expansion of the mouse OFT, although at later developmental stages (Jaffe et al., 2012). Therefore, I speculate that ECs could start the production of an ECM environment that allows for the initial OFT development. Subsequently, the control of this process could be taken over by SMCs. Additionally, the EC hyperproliferation observed in alk.5 mutants could reflect a less mature state of the vessel, therefore preventing the addition of SMCs to the OFT (Stratman et al., 2017; Sweeney and Foldes, 2018). The reduced SMC coverage could later promote EC proliferation in a positive feedback loop and aggravate the OFT expansion. Therefore, further in vivo studies in different models are needed to dissect the distinct roles of the multiple receptors in both cell types, taking into account their reciprocal interactions. (Certain lines in this subsection have been quoted verbatim from Boezio et al., eLife 2020;9:e57603 for the scientific accuracy of the terms.) 


\section{Discussion}

\subsubsection{Revisiting the role of TGF- $\beta$ in aortic diseases}

Most of the literature focusing on aortic diseases aimed to unravel the role of the TGF- $\beta$ pathway in SMCs, overlooking its function in ECs (Choudhary et al., 2009; Gillis et al., 2013; W. Li et al., 2014; P. Yang et al., 2016; Zhang et al., 2016; Michel et al., 2018). In particular, it has been shown that SMC-specific mutation in TGF- $\beta$ related genes (including Alk5) lead to aortic expansion and aneurysms postnatally (W. Li et al., 2014; P. Yang et al., 2016; Zhang et al., 2016), and these defects are considered to be a destabilization of the SMC wall (Perrucci et al., 2017). Very recently, two studies started evaluating new perspectives and a potential therapeutic role for ECs in the pathogenesis of the aneurysms (van de Pol et al., 2017; J. Sun et al., 2018). Our data support this hypothesis and identify the EC hyperproliferation as the first process leading to OFT expansion. It is tempting to speculate that, also in human patients, aortic diseases could originate from early endothelial dysfunction, masked by the major impairment of the smooth muscle wall.

The association of aneurysm formation and loss-of-function mutations in members of the TGF- $\beta$ signaling (Loeys et al., 2006; Takeda et al., 2018) suggests a downregulation of the pathway in the affected aortic specimens. However, several studies seem to contradict this hypothesis. Different mouse and human models of TGF- $\beta$ mutations exhibited hallmarks of enhanced TGF- $\beta$ signaling, as increased p-Smad staining or upregulation of target genes (Wong et al., 2000; Jones et al., 2009; F. Lin and Yang, 2010; Mallat et al., 2017). Based on these data, it was long proposed that the leading cause of aortic dilation relied on the upregulation of the TGF- $\beta$ pathway. In contrast with these findings, the use of TGF- $\beta$-neutralizing antibody predisposes mice to aneurysm formation (Y. Wang, Ait-Oufella, et al., 2010). These data argue against the enhanced TGF- $\beta$ signaling as the primary cause of aneurysms formation. To date, the molecular mechanisms of this paradox are still not understood.

Here, I showed that zebrafish alk 5 mutants exhibit enhanced TGF- $\beta$ signaling when the OFT expansion is already severe, recapitulating the mammalian data. In contrast, before the onset of the OFT phenotype, the p-Smad3 staining in alk. $5^{\%}$ hearts was reduced, as one would expect. Moreover, a specific Smad3 inhibition was able to induce a mild OFT phenotype in alk $5^{+/}$ zebrafish and to aggravate the phenotype of alk $5^{\%}$ larvae. These results together suggest that the early downregulation of the signaling in ECs could be the initial driving force leading to OFT expansion. I speculate that several feedback loops would take place following the onset of the defects to subsequently enhance the signaling.

One intriguing possibility could be that the nonfunctional TGF- $\beta$ receptors, while they cannot phosphorylate Smads directly, maintain the ability to form signaling platforms with other proteins. For instance, it has been shown that phosphatidylinositol 3 kinase (PI3K) can indeed phosphorylate Smad3 in response to TGF- $\beta$, at sites different from those phosphorylated by 
the canonical TGF- $\beta$ signaling (Runyan et al., 2004). Additional studies are needed to address the exact molecular mechanism driving aortic expansion and aneurysm formation, and the zebrafish model could offer important insights into this complex signaling pathway. (Certain lines in this subsection have been quoted verbatim from Boezio et al., eLife 2020;9:e57603 for the scientific accuracy of the terms.)

\subsection{The epicardium-myocardium crosstalk is essential for cardiomyocyte volume increase during zebrafish cardiac development}

The adhesion of epicardial cells to the myocardium takes place in a very dynamic moment during cardiac development when several morphogenetic processes directed at increasing heart size and complexity are happening. The role of the epicardium as a trophic signaling center for the myocardium during cardiac repair or coronaries development has been extensively studied and reviewed (Olivey and Svensson, 2010; Masters and Riley, 2014). Conversely, its crucial dialogue with the myocardium during cardiac development has received much less attention. Here, leveraging the advantages of three newly-established different models of epicardial impairment, I propose a new role for the epicardium in promoting CM size growth and in modulating ventricular morphogenesis.

\subsubsection{Generation of three different models of epicardial impairment: similarities and differences}

The study of epicardial contribution to heart development and homeostasis has relied on single mutant models for epicardial genes in mice (Moore et al., 1999; Merki et al., 2005; Acharya et al., 2012), or the ablation of the PEO in chick embryos (Manner, 1993; Pennisi et al., 2003; Manner et al., 2005; Takahashi et al., 2014). By using a single mutant model, it is challenging to distinguish between the specific protein role and the role of the epicardium as a whole during cardiac morphogenesis. Conversely, the PEO ablation constitutively abolished the presence of the epicardial barrier, making it difficult to separate its structural and signaling contributions. Therefore, I aimed at generating partially independent models of epicardial impairment to compare their effects on myocardial development. Notably, I showed that while the mutations in $\operatorname{tcf} 21$ and $w t 1 a$ both cause a reduction in the epicardial coverage of the ventricle, the two models exhibited important differences. While wt1 a null larvae exhibited a complete loss of the epicardium, tcf21 null larvae displayed a wide range of epicardial coverage, with some of the larvae even showing a fully covered ventricle. This difference also reflects mouse findings, in which $\operatorname{Tcf} 21 \%$ hearts show only a mildly disturbed epicardial formation (Acharya et al., 2012), while $W t 1$ mutants display substantial gaps in the epicardial lining (Moore et al., 1999). Another difference between the two models resides in the origin of the defect. While tcf 21 mutants have no initial impairment in the number of PEO cells, the mutation in wt1 a seems to affect the early 


\section{Discussion}

formation of the PEO. Further studies will be needed to further elucidate the discrepancies between the two mutants and the exact origin of their epicardial defects.

The epicardium is a very heterogeneous population, with several markers labeling nonoverlapping subset of cells already in the PEO (Braitsch et al., 2012; Katz et al., 2012; Plavicki et al., 2014; Weinberger et al., 2020). In zebrafish, most of the epicardium derives from the main PEO, while the OFT epicardial cells originate from the pericardium (Perez-Pomares et al., 2003; Peralta et al., 2014). Of note, while tcf21 and $w t 1$ a mutants show impairment in the ventricular coverage, they correctly form the OFT epicardial cells. This result further supports previous reports, showing that different molecular processes specifically affect particular subsets of cells.

In order to complement our study and achieve a temporal regulation of the epicardial loss, I refined the previously described epicardial ablation model (Nagelberg et al., 2015; J. Wang et al., 2015). Interestingly, this model allowed us to achieve a temporal resolution in dissecting the epicardial role during myocardial morphogenesis and to identify a time window important for epicardium-myocardium communication. This tool ablates nearly all the ventricular epicardial cells and the results obtained are not dependent on a particular gene's function. Thus, the NTR/Mtz ablation complements the two genetic models. Future investigations of later epicardial processes in zebrafish (e.g., the onset of EMT and EPDCs formation) will greatly benefit from this tool, which does not affect the initial phases of epicardium specification. This tool can be further expanded to study larval epicardial regeneration and understand the origin of the epicardial cells repopulating the heart after ablation. Overall, I envision that these three models will not only be important for the identification of common myocardial defects but will also be critical to uncovering important differences in epicardial formation and function.

\subsubsection{The role of the epicardium in regulating $\mathrm{CM}$ volume increase and proliferation}

During heart development, inductive signals from the surrounding tissues have been postulated to pattern the myocardial wall. Endocardial and epicardial factors promote CM proliferation, regionalization in compact and trabecular layers, maturation, and function. The role of the endocardium-myocardium signals has been extensively described (Colliva et al., 2020), while no full consensus regarding the cellular processes regulated by the epicardium during early heart morphogenesis has been reached. Nonetheless, all the models of epicardial impairment exhibit reduced cardiac size and ventricular wall thinning, strongly suggesting a role of the epicardium during cardiac growth. This role has been conventionally attributed to its regulation of CM proliferation, based on results obtained by loss-of-function mouse models (Lavine et al., 2005; P. Li et al., 2011), in vitro experiments (T. Chen et al., 2002), or some PEO ablation models 


\section{Discussion}

(Pennisi et al., 2003). Nonetheless, some studies challenged this hypothesis, as they did not observe defects in CM cycling (Kastner et al., 1994; Takahashi et al., 2014). Here, in all three models of epicardial impairment, I show no alteration in the CM number at 96 hpf or defects in CM proliferation. Of note, several of the previous studies emphasizing the defect in CM proliferation were conducted in vitro or based on single global knock-out models, not discerning the potential role of these genes in other tissues (Lavine et al., 2005; P. Li et al., 2011). Moreover, in the in vivo models, proliferation assays were performed following the onset of epicardial EMT (T. Chen et al., 2002; Pennisi et al., 2003; Lavine et al., 2005), thereby later than the timing of my analyses.

Of note, the defect in CM proliferation observed by Pennisi et al. following PEO ablation was extremely mild and also accompanied by an increase in cell density (Pennisi et al., 2003), similar to what I observed in the three zebrafish models at 96 hpf. Interestingly, Takahashi et al., using a similar PEO surgical ablation technique, partially contradicted the interpretation of the previous results. In fact, they linked the increased CM density to a reduced cellular area, and not with an impaired proliferation (Takahashi et al., 2014). Nonetheless, this study fails to investigate CM growth over time and only considers two-dimensional CM areas on sections as a proxy for cell size. Here, I present evidence for impaired CM growth by measuring CM volume increase over time in the same wild-type or $\operatorname{tcf} 21$ mutant larvae. Coupled with the reduced $\mathrm{CM}$ area, and no sign of reduced CM number, these results suggest that the first process affected following epicardial loss is CM volume growth.

Additionally, I hypothesize that the drop in volume increase might influence subsequent cell cycles. The dependency of proliferation on cell size has been widely recognized in different cellular models (Jorgensen and Tyers, 2004). Moreover, it has been described that cells including CMs- swell and enlarge prior to division both in vitro and in vivo (Son et al., 2015; Zlotek-Zlotkiewicz et al., 2015; Uribe et al., 2018). Therefore, further experiments will be needed to address CM proliferation and number at later stages of heart development in the epicardial-deficient larvae. These studies will help to shed light on CM behavior and the relationship between hyperplastic and hypertrophic growth driving ventricular morphogenesis.

\subsubsection{The epicardium as a signaling center}

Early embryologists until the beginning of the $20^{\text {th }}$ century considered the epicardium as an inert barrier, simply protecting the naked myocardium from the pericardial fluids. It is now widely accepted the relevance of this monolayer as an important signaling center (Quijada et al., 2020). Nonetheless, the appearance of $\mathrm{CM}$ extrusions in all the three zebrafish models of epicardial impairment, similar to findings in chick following PEO ablation (Manner et al., 2005), newly raised the question about the importance of the epicardium as mechanical support. However, 


\section{Discussion}

here I show that even the presence of a complete epicardial layer in tcf 21 mutants does not prevent the abluminal extrusion of CMs, nor improves the volume growth. This finding suggests that the sole epicardial coverage is not sufficient to promote ventricular morphogenesis when its function is affected. I envision that tcf 21 mutant larvae showing a consistent epicardial coverage will be key in answering several questions about the epicardial processes and signaling cues necessary for myocardial growth.

Despite the acclaimed function of the epicardium as a signaling hub (Olivey and Svensson, 2010; Masters and Riley, 2014), few factors are currently known to play a role in the crosstalk to the myocardium and this topic has not been revisited in the last 5 years. Among the important paracrine secreted factors, we can mainly name IGF and FGF family members. Especially for the FGF pathway, the concomitant endocardial expression of several FGF genes complicates the interpretation of the results. For instance, FGF9, -16, -20 are secreted by both the endocardial and epicardial layers, and the very early lethality of FGF16 null embryos strongly implicates their endocardial function in regulating cardiac trabeculation (Lavine et al., 2005; Lu et al., 2008). Moreover, the cardiac phenotype of the myocardial-specific FGFR1 and FGFR2 mutants affects only a portion of the ventricle, suggesting the cooperation of different ligand/receptors during cardiac development (Lavine et al., 2005).

Therefore, many of the factors mediating the epicardium-myocardium communication still need to be identified, and the exact function of known paracrine signaling in this crosstalk requires further investigation. To date, very few comprehensive transcriptomic or proteomic datasets are available to dissect this process. An interesting proteomic analysis of the epicardial secretome was recently published by $\mathrm{Li}$ et al., which identifies several of the known pathways and adds novel factors to the list (Y. Li et al., 2017). Nonetheless, this dataset was obtained by cardiac explants and not in physiological in vivo settings. In our dataset, I identified several epicardial-enriched genes previously acknowledged (such as FGF, IGF, PDGF family members), corroborating the previous literature and showing the similarity of mammalian and zebrafish models in this process. Moreover, I observed the epicardial enrichment of new potential candidates, including Angiopoietin-like genes, Follistatin, and Tumor necrosis factors, which will be interesting to characterize.

Among the genes downregulated in the tcf 21 mutant epicardium, I focused mainly on two paracrine factors, fof 24 and vegfaa. The FGF pathway and its downstream ERK signaling have renowned importance in the epicardium-myocardium crosstalk, but we still lack information about the zebrafish ligand-receptors involved in this process. $f g f 24$ is the mostly enriched FGF gene in the epicardium and is the ortholog of mammalian Fgf18, which also shows a restricted epicardial expression (Hagan et al., 2019). Interestingly, Fgf24 could bind the receptor Fgfr4 on the CM membrane, as suggested by the myocardial enriched expression of this receptor. As 


\section{Discussion}

previously described, the role of FGF only focused on its mitogenic function on CMs. My results obtained with the small molecule inhibitor suggest a role in CM growth, but the exact cellular process downstream of the ERK activation requires further investigation.

Another pathway relying on the downstream ERK signaling is VEGF. The expression pattern of the ligand Vegfa in mouse and zebrafish has shown several discrepancies (Tomanek et al., 1999; Lavine et al., 2006; B. Wu et al., 2012; Karra et al., 2018), and its epicardial role was never described. I proved that in zebrafish larval stages vegfaa is initially expressed only in the epicardium (up to $4 \mathrm{dpf}$ ). Previous reports describe the appearance of its expression in a few endocardial cells from $7 \mathrm{dpf}$ (Karra et al., 2018). Nonetheless, the lack of enriched receptors on the myocardial side raises questions about the Vegfaa signaling mechanism during cardiac morphogenesis. Several factors have been identified to act in an autocrine manner in the epicardium, such as Epo and RA, coordinating the secretion of downstream paracrine factors, like FGF and IGF (Stuckmann et al., 2003; Brade et al., 2011). Therefore, I speculate that the zebrafish Vegfaa, signaling through the epicardial-enriched Kdrl receptor, might function in a similar fashion. Otherwise, it might bind to alternative myocardial receptors and contribute to the amplification of the downstream ERK signaling in CMs. Interestingly, it has been described that overexpression of vegfaa in CMs causes cardiomegaly, driven by increased CM number (Laguens et al., 2004; Karra et al., 2018). This result, together with my findings obtained with the VEGFR inhibitor, suggests a role of Vegfaa in promoting cardiac growth. Karra et al suggest a likely role for the endocardium as a mediator of the Vegfaa effects. The authors mainly based their interpretation on the results obtained in adult stages, when the endocardial expression of vegfaa is prominent (Karra et al., 2018). Instead, based on the expression pattern of vegfaa in larval stages, I imagine that -during development- Vegfaa might act from epicardial cells. It will be key for future studies to identify the exact mechanisms of action and the contribution of VEGF and FGF to CM proliferation and volume increase in the myocardial wall. 


\section{Conclusions}

\section{Conclusions}

In conclusion, I characterized important hubs of cellular interactions during zebrafish heart development and provided evidence of the signaling pathways mediating their cross-talk.

Broadly, I described with genetic and high-resolution imaging tools the communication of different kinds of non-muscular cells with the cardiac muscles and proved the relevance of these interactions for cardiac morphogenesis. In particular, both epicardial (Aim1b) or endothelial defects (Aim1a) affect muscle cell-specific processes, which drive severe cardiac malformations leading to impaired cardiac function. I also identified different kinds of signaling interactions between these cell types, mediated by the extracellular environment (Aim1a) and by paracrine factors (Aim1b).

\section{Aim 1a: Endothelium-smooth muscle crosstalk in the cardiac outflow tract}

I identified in the developing OFT an important center of inter-tissue crosstalk and improved our understanding of its development, cellular contributions, and signaling pathways. Specifically, the TGF- $\beta$ pathway plays a pivotal role in the morphogenesis of this structure, by primarily acting on endothelial cells. After deletion of alk5, embryos showed a specific dilation of the OFT caused by a multicellular defect. In particular, I provided evidence of the role of Alk5 in restricting EC proliferation, promoting SMC proliferation, and modulating the ECM organization. By fine-tuning the extracellular environment, I hypothesize that Alk5 orchestrates the signaling between the two neighboring tissues. These data were strongly supported by highresolution live-imaging coupled with tissue-specific overexpression of alk.5 and the ECMcomponent gene $f b \ln 5$, which were able to rescue the alk 5 mutant phenotype.

\section{Aim 1b: Epicardium-myocardium crosstalk in the cardiac ventricle}

In the second part of this work, I investigated the crosstalk between epicardium and myocardium in the zebrafish larval hearts. I generated three independent models of epicardial impairment and combined them to elucidate a common effect of the epicardium on myocardial morphogenesis. Intriguingly, the lack of epicardium caused a drop in CM volume increase over time, without affecting $\mathrm{CM}$ number or proliferation. This effect proved to be very timedependent since the ablation of the epicardium in late larval stages did not cause any defect in ventricular growth. Lastly, I generated a dataset to describe epicardial-specific secreted factors important to drive epicardium-myocardium cross-talk. I identified defective epicardial-derived FGF and VEGF signaling in tcf 21 mutants, and I propose that the downregulation of the common downstream ERK pathway in CMs might be partially responsible for the ventricular defects. 


\section{Conclusions}

These studies performed in vivo, provide new insights into the mechanisms of cellular communication in the heart, by contributing with new models to the study of these interactions and identifying unexpected roles for non-muscular tissues in regulating cardiac development. A clearer understanding of these cell-to-cell and cell-to-ECM crosstalks may also provide potential novel therapeutic targets for congenital heart defects and cardiac regeneration. 


\section{$7 \quad$ Summaries}

\subsection{Zusammenfassung}

Verständnis der Interaktionen zwischen den Geweben während der kardiovaskulären Entwicklung des Zebrafisches

Das kardiovaskuläre System besteht aus einem Herz und Blutgefäßen, die den gesamten Organismus mit Sauerstoff und Nährstoffen versorgen. Das Herz ist das erste Organ, das sich während der Eembryonalentwicklungn bildet, wobei die ersten Vorläufer bereits während der Gastrulationsstadien spezifiziert werden (Keegan et al., 2004; Ivanovitch et al., 2020). Die kardialen Vorläuferzellen wandern zur Mittellinie, wo sie konvergieren und den primitiven Herzschlauch bilden, der hauptsächlich aus zwei Geweben besteht. Das Endokard, gebildet aus spezialisierten Endothelzellen (ECs), kleidet das Lumen aus, während das Myokard, bestehend aus Kardiomyozyten (CMs), das Endokard bedeckt und für die Herzkontraktion verantwortlich ist (Schleich et al., 2013; Brown et al., 2016). Sobald der Herzschlauch gebildet ist, finden viele morphogenetische Ereignisse statt, um seine Größe, Komplexität und funktionelle Effizienz zu erhöhen. Am arteriellen Pol des Ventrikels wird der Kanal des Ausflusstrakts (OFT) gebildet, um den hohen Blutdruck abzupuffern. Die Bildung der atrioventrikulären (AV) und der bulboventrikulären (BV) Klappen sorgen für einen unidirektionalen Blutfluss, und ventrikuläre Trabekel ragen im Inneren der Kammern hervor, um die Herzkontraktion zu unterstützen (Schleich et al., 2013; Kelly et al., 2014).

Diese Vorgänge erfordern zelluläre Prozesse wie Proliferation, Spezifikation sowie Reifung bestehender Zellen. Die Komplexität des Herzens entsteht jedoch vor allem durch das kontinuierliche Hinzufügen neuer Zelltypen während seiner Reifung. Nach der Entwicklung des Herzschlauchs werden neue Vorläuferzellen aus dem zweiten Herzfeld am arteriellen Pol des Herzens hinzugefügt, wodurch zusätzliche CMs und ECs entstehen (Buckingham et al., 2005; Meilhac et al., 2014). Bei E9-E9,5 in der Maus und 52 hpf (hours post fertilization/ Stunden nach Befruchtung) im Zebrafisch beginnt das Epikard zu den Kammern beizutragen, indem es sich vom extrakardialen proepikardialen Organ (PEO) ablöst (Manasek, 1969; Manner et al., 2001; Simoes und Riley, 2018; Quijada et al., 2020). Epikardiale Zellen haften an der Myokardoberfläche, proliferieren und bedecken die Herzkammern. Sie tragen später auch zu Koronargefäßen und Fibroblasten im erwachsenen Herzen bei (Simoes und Riley, 2018; Quijada et al., 2020). Zusätzlich werden glatte Muskelzellen (SMCs) für die Bildung von OFT umgebenden ECs rekrutiert, um den Gefäßtonus und den Blutdruck zu regulieren (Mjaatvedt et al., 2001; Grimes et al., 2010). Ein weiterer Zelltyp, der zur Morphogenese des Herzens beiträgt, sind die vom Ektoderm stammenden Neuralleistenzellen (NCCs) (Hutson und Kirby, 
2003; Stoller und Epstein, 2005; Plein et al., 2015). Keiner dieser Zelltypen ist von den anderen isoliert, und ihre Kommunikation ist entscheidend für die Koordination der Herzentwicklung (Tirziu et al., 2010; Fountoulaki et al., 2015). Benachbarte Zellen können physisch leicht durch zellkontaktvermittelte Interaktionen kommunizieren.

Umgekehrt sind die Zellen, wenn sie nicht benachbart sind, auf die Sekretion von Faktoren in die Blutbahn angewiesen. Unter diesen Faktoren befinden sich Liganden der gängigsten Entwicklungssignalwege, wie z. B. vaskulärer endothelialer Wachstumsfaktor (VEGF), Fibroblasten-Wachstumsfaktor (FGF), knochenmorphogenetisches Protein (BMP)/ TGF- $\beta$, PDGF und einige andere (Fountoulaki et al., 2015). Ein weiterer Weg für Zellen, miteinander in Kontakt zu treten, ist die extrazelluläre Matrix (EZM), ein Geflecht aus verschiedenen Fasern (Kim et al., 2011). Die EZM spielt auch eine entscheidende Rolle bei der Etablierung der richtigen extrazellulären Mikroumgebung für die Zellkommunikation, reguliert das Zellverhalten und die Genexpression und bietet gleichzeitig strukturelle Unterstützung. Außerdem dient die EZM als Reservoir für Wachstumsfaktoren, wie TGF- $\beta$, BMP und FGFs (Kim et al., 2011; Valiente-Alandi et al., 2016).

Für das Verständnis der interzellulären Kommunikation in einer solch komplexen und dynamischen Umgebung könnte die zelluläre Live-Bildgebung von großem Nutzen sein, da dieses erlaubt, die Entwicklung über die Zeit zu verfolgen. Die größten Herausforderungen bei der Untersuchung der interzellulären Kommunikation im sich entwickelnden Säugetierherz liegen in der frühen Letalität von Embryonen mit kardialen Phänotypen, gepaart mit der internen Entwicklung, die Live-Bildgebung verhindert. Der Zebrafisch hat sich als exzellentes Modell erwiesen, da er sich für hochauflösende Live-Bildgebung eignet und mehrere Tage ohne funktionierendes Herz überleben kann. Daher habe ich in dieser Studie die Vorteile des Zebrafischmodells genutzt, um zelluläre Interaktionen während der Larvenentwicklung des Zebrafisches zu analysieren, wobei ich mich auf zwei spezifische kardiale Prozesse konzentrierte, die bei Säugetieren nicht vollständig charakterisiert sind.

Im ersten Teil dieser Studie charakterisierte ich den zellulären und molekularen EC-SMCCrosstalk, der die Entwicklung des kardialen OFT orchestriert, und seine Abhängigkeit vom TGF- $\beta$-Signalweg. Zunächst habe ich die Expression und die Aktivierung des TGF- $\beta$ Signalwegs im sich entwickelnden OFT charakterisiert und mich dabei auf den Alk5-Rezeptor konzentriert. Interessanterweise konnte ich beobachteten, dass Alk5 zwar während der Entwicklung im gesamten Herzen exprimiert wurde, die nachgeschaltete Aktivierung des Signalwegs aber zunächst konsequent in OFT ECs angereichert war. Später wechselte diese bevorzugte Aktivierung progressiv von ECs zu SMCs. Diese Ergebnisse deuten auf das Vorhandensein mehrerer Regulationsmechanismen downstream von Alk5 hin, die die präzise zeit- und raumspezifische Aktivierung des Signals orchestrieren. 


\section{Summaries}

Als nächstes habe ich versucht, die Funktion von Alk5 während der OFT-Entwicklung des Zebrafisches zu untersuchen, indem ich alk5a- und alk.5b-Doppelmutanten (Paraloge des Säugetier-Alk5) mit Hilfe der CRISPR/Cas9-Technologie erzeugt habe. Als ich die Herzmorphologie in alk5-Mutanten analysierte, konnte ich eine extrem spezifische OFTErweiterung beobachten, die bei $54 \mathrm{hpf}$ begann und sich bei $78 \mathrm{hpf}$ verschlimmerte, was zu einer gestörten Blutzirkulation führte. Gelegentlich wiesen alk5-Mutanten Rupturen in der OFT-EC-Schicht auf, die den Aortenaneurysma-Dissektionen bei menschlichen Patienten ähneln (Takeda et al., 2018). Menschliche Patienten, die von thorakalen Aortenaneurysmen und Dissektionen betroffen sind, weisen häufig Alk5-Loss-of-Function-Mutationen auf, obwohl die Veränderung der nachgeschalteten Signale die zu diesen Defekten führt, noch sehr umstritten ist (Jones et al., 2009; Lin und Yang, 2010). Um den TGF- $\beta$-Downstream-Signalweg in alk:5-Mutanten zu untersuchen, habe ich die Phosphorylierung des intrazellulären Effektors Smad3 untersucht. Bemerkenswerterweise beobachtete ich eine signifikante Herunterregulation des Signalwegs, insbesondere in ECs, bei 24 hpf, während bei 74 hpf (d.h. wenn der OFTPhänotyp bereits schwerwiegend ist) alk-5-Mutanten eine überraschende Hochregulation aufweisen. Bemerkenswert ist, dass die Behandlung von alk $5^{-1}$-Embryonen mit einem spezifischen Smad3-Inhibitor die OFT-Defekte verschlimmerte. Insgesamt deuten diese Daten darauf hin, dass eine initiale TGF- $\beta$-Herunterregulation für die Ätiologie der OFT-Dilatation verantwortlich ist, aber sekundäre Rückkopplungsschleifen nach dem Auftreten des Phänotyps stattfinden.

Als nächstes habe ich den OFT-Phänotyp auf zellulärer Ebene charakterisiert. Mit hochauflösendem Live-Imaging beobachtete ich, dass $78 \mathrm{hpf}$ alk.5 mutierte OFTs aus doppelt so vielen ECs im Vergleich zum Wildtyp bestanden, während bei 36 hpf die Anzahl der ECs zwischen den beiden Genotypen vergleichbar war. Mit Hilfe eines EdU-Aufnahmeassays beobachtete ich, dass alk 5 ECs überraschenderweise bereits vor dem Auftreten eines morphologischen Defekts (24-36 hpf) vermehrt proliferierten. Dieses Experiment legt nahe, dass das Auftreten der OFT-Defekte mit der ersten beobachtbaren Aktivierung des TGF- $\beta$ Signalwegs in OFT-ECs zusammenfällt.

Eine übermäßige Ausdehnung des OFT ist oft mit einer defekten SMC-Wand verbunden. Daher habe ich die Bildung der SMC-Wand in alk5-Mutanten bei 75 hpf durch LiveBildgebung, Immunfärbungen und Transmissionselektronenmikroskopie (TEM) analysiert. Ich beobachtete, dass alk 5/- OFTs im Vergleich zu Wildtypen von einer reduzierten Anzahl von SMCs umgeben waren. Im Gegensatz zu den ECs schienen die SMCs weniger proliferativ zu sein, wenn Alk5 fehlte. Die TEM-Aufnahmen zeigten, dass alk.5 $5^{-1}$ OFT-Wände auch breitere extrazelluläre Räume aufwiessen, und die Elastin2-Immunfärbung bestätigte eine beeinträchtigte EZM-Umgebung. 


\section{Summaries}

Um zu verstehen, welcher Zelltyp für den OFT-Phänotyp verantwortlich ist, überexprimierte ich Wildtyp-alk $5 b$ spezifisch in ECs, indem ich das Tg(fli1a:alk-5b-P2A-mScarlet) Konstrukt injizierte. Während die EC-spezifische Überexpression bei Wildtyp-Tieren keinen Defekt verursachte, rettete sie den Phänotyp der alk5-Mutante sowohl morphologisch als auch funktionell vollständig. Überraschenderweise stellte die alleinige EC-Rettung nicht nur die ECProliferation in alk5-Mutanten wieder her, sondern führte auch zu einer vollständigen Rettung der SMC-Proliferation und EZM-Zusammensetzung. Diese Ergebnisse deuten stark darauf hin, dass Alk5 in ECs ausreichend vorhanden ist, von wo aus es die interzelluläre Kommunikation mit SMCs orchestriert.

Um den Ursprung und die Natur dieser Interaktion auf molekularer Ebene besser zu charakterisieren, führte ich Analysen des Transkriptoms von 56 hpf extrahierten Herzen nach Alk5-Inhibition durch. Ich beobachtete, dass unter den verschiedenen biologischen Prozessen und zellulären Kompartimenten die EZM am stärksten betroffen war. Ich identifizierte und untersuchte das Kandidatengen $f b \ln 5$, das ein Integrin-bindendes Protein codiert, das sich als ein sehr spezifischer OFT-Marker im embryonalen Zebrafisch erwies. Bemerkenswert ist, dass die EC-spezifische Überexpression von $f b \ln 5$ in der alk5-Mutante die EC-Proliferation, die Präsnz von Eln2 und die OFT-Expansion teilweise wiederherstellen konnte. Diese Daten deuten auf eine Rolle von Fbln5 downstream von Alk5 bei der Vermittlung der EC-SMCInteraktion hin, möglicherweise durch Förderung der EC-Anhaftung an die Matrix, wodurch ihre Proliferation gehemmt wird (Sullivan et al., 2007; Williamson et al., 2007), und durch Unterstützung der in vivo Elastogenese (Nakamura et al., 2002). Insgesamt schlagen ich in diesem Teil der Studie ein Modell vor, in dem Alk5 die EC-SMC-Kommunikation im kardialen OFT vermittelt, indem es dessen EZM-Umgebung reguliert.

Im zweiten Teil dieser Studie wollte ich die Interaktionen zwischen Epikard und Myokard im Larvenherz des Zebrafisches untersuchen. Die Interaktion zwischen Epikard und Myokard und ihre Rolle bei der kardialen Reparatur wurde bei erwachsenen Mäusen und Zebrafischen ausgiebig untersucht, wobei der Schwerpunkt auf dem Beitrag neuer Zelltypen und mitogener Faktoren lag (Pennisi et al., 2003; Smits et al., 2018; Quijada et al., 2020). Nichtsdestotrotz gibt es zwar Hinweise auf die Rolle des Epikards in der frühen kardialen Entwicklung und seine Umhüllung des Herzens fällt zeitlich mit wichtigen myokardialen morphogenetischen Ereignissen zusammen, aber sein Einfluss auf das CM-Verhalten erfordert eine weitere Charakterisierung.

Daher habe ich versucht, Live-Bildgebung mit verschiedenen Modellen zu kombinieren, in denen die Bildung der Epikarddecke gestört ist, um die unterschiedlichen Rollen dieses Gewebes bei der Aufrechterhaltung der kardialen Morphogenese zu klären. 


\section{Summaries}

Unter den bekannten epikardialen Markern mutierten wir die Transkriptionsfaktor-Gene tcf21 und $w t 1 a$. Durch Live-Bildgebung der transgenen Linie Tg(tcf21:nls-EGFP) und pan-epikardiale Immunfärbung konnte ich beobachten, dass beide Mutationen in tcf21 und wt1a die Epikardhülle des Ventrikels reduzierten. Bemerkenswerterweise zeigen wt1a-Mutanten ein komplettes Fehlen von $t c f 21^{+}$Zellen, die dem Ventrikel anhaften, während tcf21-Mutanten von einem kompletten Verlust bis zu einem Niveau ähnlich dem des Wildtyps reichten. Um die Studie zu ergänzen, habe ich versucht, ein zeitlich reguliertes Modell der gestörten epikardialen Bedeckung zu erhalten. Ich habe $t c f 21^{+}$-Epikardzellen spezifisch unter Verwendung des bakteriellen Enzyms Nitroreduktase (NTR) ablatiert, das bei Verabreichung seines Substrats Metronidazol (Mtz) spezifisch Apoptose induziert. Die Behandlung von Tg(tcf21:mCherryNTR)-Larven (Nagelberg et al., 2015, Wang et al., 2015) von 54 bis 100 hpf phänokopierte das in den beiden genetischen Modellen beobachtete Fehlen der Epikardabdeckung. Insgesamt bilden diese drei teilweise unabhängigen Modelle eine grundlegende Basis, um den Effekt des fehlenden Epikards auf die Herzmorphogenese zu untersuchen.

Ich beobachtete, dass bei Abwesenheit des Epikards die 96 hpf-Larven aller drei Modelle eine reduzierte Herzgröße aufwiesen, ein Defekt, der in früheren Stadien nicht beobachtet wurde. Bemerkenswert ist, dass das ventrikuläre Volumen in Wildtyp-Tieren zwischen 72 und 96 hpf zunahm, während es in tcf21- und wt1a-Mutanten im Laufe der Zeit nicht wuchs. Die Zunahme der Organgröße kann durch hyperplastisches und/oder hypertrophisches Wachstum angetrieben werden, wenn es durch eine Zunahme der Zellzahl bzw. Zellgröße gesteuert wird. Um die zellulären Prozesse zu verstehen, die in Abwesenheit des Epikards betroffen sind, habe ich daher zunächst die CM-Anzahl und -Proliferation analysiert und festgestellt, dass sie in keinem der Modelle betroffen waren. Um das hypertrophe Wachstum der CM zu untersuchen, verfolgte ich spezifische CMs über die Zeit in $t c f 21^{+/+}$und $t c f 21^{\%}$ Herzen, die myl7:mScarlet mosaik exprimierten. Durch Messung des einzelnen CM-Volumens beobachtete ich, dass dieses in $t c f 21^{+/+}$Herzen zwischen 76 und 100 hpf um 25,7 \% zunahm, während sich das tcf $21^{-}$

${ }^{1} \mathrm{CM}-$ Volumen nicht veränderte $(+1,4 \%)$. Insgesamt deuten meine Beobachtungen auf eine frühe Rolle des Epikards bei der Förderung des hypertrophen Wachstums während der ventrikulären Entwicklung hin.

Um die Abhängigkeit des beeinträchtigten Herzwachstums vom Fehlen des Epikards weiter zu untersuchen, nutzte ich die zeitliche Flexibilität des NTR/Mtz-Systems. Ich stützte mich auf die Fähigkeit des Epikards, sich nach einer Verletzung zu reaktivieren und zu regenerieren (Wang et al., 2015; Cao und Poss, 2018), um zu verstehen, ob die Wiederherstellung der Epikardhülle ausreichen würde, um das ventrikuläre Wachstum zu verbessern. Mit diesem Ansatz beobachtete ich, dass die epikardiale Regeneration von 4 bis 6 dpf (days post 


\section{Summaries}

fertilization/Tage nach Befruchtung) ausreichend war, um das ventrikuläre Volumen signifikant zu verbessern.

Als nächstes habe ich versucht, epikardiale Faktoren zu identifizieren, die die Interaktionen mit dem Myokard regulieren. Daher nutzte ich die tcf21-Mutanten, in denen trotz einer morphologisch fast normalen Epikardhülle Defekte im Ventrikelwachstum auftraten, ähnlich den Defekten nach einer Ablation der Epikardhülle. Die fehlende Korrelation zwischen dem ventrikulären Volumen und der Anzahl der tcf $21^{+}$Zellen am Ventrikel in diesen Mutanten bestätigte, dass das Epikard nicht als mechanische Barriere ausreicht, sondern benötigt wird, um Faktoren bereitzustellen, die für das myokardiale Wachstum essentiell sind. Um die fehlenden epikardialen Faktoren zu verstehen, die für eine korrekte myokardiale Entwicklung in tcf21-Mutanten notwendig sind, führte ich Analysen des Transkriptoms sortierter $t c f 21^{+/+}$und tcf21/- Epikardzellen und CMs im 96 hpf- Stadium durch. Der Wildtyp-Epikarddatensatz ist stark angereichert für die erwarteten Signalwege, wie FGF, TGF- $\beta$ und PDGF, was darauf hindeutet, dass mein Datensatz die molekularen Prozesse widerspiegelt, die an der EpikardMyokard-Kommunikation beteiligt sind. Unter den Genen, die in $t c f 21^{\%}$ Herzen spezifisch herunterreguliert sind, sind die sekretierten Faktoren vegfa $a$ und $f g f 24$ von besonderem Interesse, da beide Signale über den gemeinsamen ERK-Weg weitergeben. Daher habe ich die Aktivierung des ERK-Signalwegs mit Hilfe einer neuartigen transgenen Biosensor-Linie in CMs nach epikardialer Ablation untersucht und konnte deren Herunterregulation beobachten. Darüber hinaus führen niedermolekulare Inhibitoren sowohl des VEGF- als auch des FGF-Signalwegs zu einem reduzierten ventrikulären Volumen in Wildtyp-Herzen, was auf ihre Beteiligung an Prozessen im Zusammenhang mit dem myokardialen Wachstum hindeutet.

Im zweiten Teil meiner Studie beschreibe ich daher eine detaillierte Charakterisierung von drei neuartigen Modellen der Epikardstörung. Meine Daten deuten auf eine unerwartete Rolle des Epikards bei der Regulation des hypertrophen CM-Wachstums während der frühen kardialen Morphogenese hin, die möglicherweise zu einem nachfolgenden Defekt der Zellteilung führt, wie er häufig in eukaryotischen Zellen beschrieben wird (Jorgensen und Tyers, 2004). In Zukunft werden Studien in diesen Modellen wichtig sein, um die molekulare Rolle der VEGFund FGF-Faktoren bei der Regulation des hypertrophen Wachstums der CM weiter zu entschlüsseln und um neue epikardiale Faktoren zu identifizieren, die die Entwicklung und Reparatur des Myokards regulieren.

\section{Schlussfolgerung}

Zusammenfassend habe ich zwei wichtige, aber noch wenig untersuchte Knotenpunkte des interzellulären Crosstalks im sich entwickelnden Herzen charakterisiert und dabei unerwartete Rollen für nicht-muskuläre Gewebe identifiziert. 


\section{Summaries}

Zunächst habe ich eine bedeutende Rolle für den TGF- $\beta$-Signalweg beschrieben, bei der Orchestrierung des EC-SMC-Crosstalks im sich entwickelnden Ausflusstrakt durch Regulation der extrazellulären Umgebung. Ich beobachtete eine bisher unbekannte Rolle für Alk5 im Endothel während der OFT-Entwicklung, die auf die Untersuchung von vaskulären Pathologien ausgeweitet werden könnte. Darüber hinaus habe ich den komplexen nachgeschalteten molekularen Signalweg, der zu den OFT-Defekten führt, charakterisiert und damit neue Erkenntnisse zur Erklärung der kontroversen Ergebnisse bei Säugetieren geliefert.

Darüber hinaus habe ich mit neuen Modellen zur Untersuchung der Epikard-MyokardInteraktion beigetragen. Analysen dieser drei Modelle haben eine neue Rolle für das Epikard, durch FGF und VEGF, bei der Förderung des hypertrophen Wachstums des CM aufgedeckt. Diese Werkzeuge liefern auch wertvolle Datensätze, die leicht erweitert werden können, um weitere Signalwege zu identifizieren. In Zukunft könnten diese Studien als Plattform dienen, um die Interdependenz zwischen hyperplastischem und hypertrophem Wachstum und die Rolle des Epikards bei diesen Prozessen zu charakterisieren. 


\subsection{English summary}

\section{Understanding inter-tissue crosstalk during zebrafish cardiovascular development}

The cardiovascular system consists of a heart and blood vessels, delivering oxygen and nutrients to the entire organism. The heart is the first organ to form during embryonic life, with the first progenitors already specified during gastrulation stages (Keegan et al., 2004; Ivanovitch et al., 2020). Cardiac precursors migrate to the midline, where they converge forming the primitive heart tube, composed of mainly two tissues. The endocardium, formed by specialized endothelial cells (ECs), lines the lumen, while the myocardium, composed of cardiomyocytes (CMs), covers the endocardium and is responsible for cardiac contraction (Schleich et al., 2013; Brown et al., 2016). Once the heart tube is formed, many morphogenetic events take place to increase its size, complexity, and functional efficiency. The outflow tract (OFT) canal is formed at the arterial pole of the ventricle to buffer the high blood pressure, the formation of the atrioventricular (AV) and the bulboventricular (BV) valves ensure unidirectional blood flow, and ventricular trabeculae protrude inside the chambers to support cardiac contraction (Schleich et al., 2013; Kelly et al., 2014).

These events require cellular processes such as proliferation, specification, and maturation of existing cells. Nonetheless, what makes the heart such a complex organ is the continuous addition of new cell types throughout its maturation. After the development of the cardiac tube, new precursors from the second heart field are added to the arterial pole of the heart, giving rise to additional CMs and ECs (Buckingham et al., 2005; Meilhac et al., 2014). At E9E9.5 in mouse and $52 \mathrm{hpf}$ (hours post fertilization) in zebrafish, the epicardium starts contributing to the chambers, delaminating from the extracardiac proepicardial organ (PEO) (Manasek, 1969; Manner, 1993; Simoes and Riley, 2018; Quijada et al., 2020). Epicardial cells adhere to the myocardial surface, and proliferate, covering the cardiac chambers. They will later also contribute to coronary vessels and fibroblasts in the adult hearts (Simoes and Riley, 2018; Quijada et al., 2020). Additionally, smooth muscle cells (SMCs) are recruited to the forming OFT surrounding ECs, to regulate vascular tone and blood pressure (Mjaatvedt et al., 2001; Grimes et al., 2010). Another type of cells that contribute to the morphogenesis of the heart is the ectoderm-derived neural crest cells (NCCs) (Hutson and Kirby, 2003; Stoller and Epstein, 2005; Plein et al., 2015).

None of these cell types is isolated from the others, and their communication is critical to coordinate cardiac development (Tirziu et al., 2010; Fountoulaki et al., 2015). Neighboring cells can readily communicate through physical interactions, via junction-mediated cell contacts. Conversely, when cells are not adjacent, they rely on the secretion of factors into the bloodstream. Among these factors, there are ligands of the most common developmental signaling pathways, such as vascular endothelial growth factor (VEGF), fibroblast growth factor 


\section{Summaries}

(FGF), bone morphogenetic protein (BMP)/ TGF- $\beta$, PDGF, and several others (Fountoulaki et al., 2015). Another way for cells to be in contact with each other is through the extracellular matrix (ECM), a meshwork of different fibers (Kim et al., 2011). The ECM also plays a crucial role in establishing the correct extracellular microenvironment for cell communication, regulating cell behavior and gene expression, while also providing structural support. Besides, the ECM serves as a reservoir of growth factors, such as TGF- $\beta$, BMP, and FGFs (Kim et al., 2011; Valiente-Alandi et al., 2016).

Understanding intercellular communication within such a complex and dynamic environment could greatly benefit from live cellular imaging, as it allows one to follow the development over time. The main challenges in studying intercellular communication in the developing mammalian heart reside in the early lethality of embryos with cardiac phenotypes, coupled with their internal development, which prevents live imaging. Zebrafish proved to be an excellent model due to its amenability to high-resolution live imaging and the possibility to survive for several days without a functional heart. Therefore, in this study, I leveraged the advantages of the zebrafish model to analyze cellular cross-talks during zebrafish larval development, focusing on two specific cardiac processes not fully characterized in mammals.

In the first part of this study, I characterized the cellular and molecular EC-SMC crosstalk orchestrating the development of the cardiac OFT, and its dependency on the TGF- $\beta$ signaling pathway. Initially, I characterized the expression and the activation of the TGF- $\beta$ signaling pathway in the developing OFT, focusing on the Alk5 receptor. Interestingly, I observed that, while alk. 5 was expressed throughout the heart during development, the downstream activation of the signaling was initially consistently enriched in OFT ECs. Later on, this preferential activation progressively switched from ECs to SMCs. These results suggest the presence of several regulatory mechanisms downstream of Alk5, which orchestrate the precise time- and space-specific activation of the signaling.

Next, I aimed at dissecting the function of Alk5 during zebrafish OFT development, by generating alk 5 a, alk.5b (paralogs of the mammalian Alk.5) double mutants, using CRISPR/Cas9 technology. When I analyzed heart morphology in alk.5 mutants, I could observe an extremely specific OFT expansion starting at $54 \mathrm{hpf}$ and becoming more severe at $78 \mathrm{hpf}$, causing defective blood circulation. Occasionally, alk.5 mutants exhibited ruptures in the OFT EC layer, resembling aortic aneurysm dissections in human patients (Takeda et al., 2018). Human patients affected by thoracic aortic aneurysm and dissection often harbor Alk5 loss-of-function mutations, although the alteration of the downstream signaling leading to these defects is still very controversial (Jones et al., 2009; Lin and Yang, 2010). To investigate the TGF- $\beta$ downstream pathway in alk5 mutants, I assessed the phosphorylation of the intracellular effector Smad3. Notably, I observed a significant downregulation of the signaling, particularly 


\section{Summaries}

in ECs, at $24 \mathrm{hpf}$, while at $74 \mathrm{hpf}$ (i.e., when the OFT phenotype is already severe) alke 5 mutants exhibit a surprising upregulation. Notably, treating alk. $5^{-1}$ embryos with a specific Smad3 inhibitor exacerbated the OFT defects. Altogether, these data suggest that an initial TGF- $\beta$ downregulation is responsible for the etiology of the OFT dilation, but secondary feedback loops happen after the appearance of the phenotype.

Next, I deeply characterized the OFT phenotype at a cellular-specific level. With highresolution live imaging, I observed that 78 hpf alk.5 mutant OFTs were composed of twice as many ECs compared with wild-type, while at $36 \mathrm{hpf}$ the number of EC was comparable between the two genotypes. Using an EdU incorporation assay, I observed that alk.5\% ECs were surprisingly more prone to proliferate already before the appearance of a morphological defect (24-36 hpf). This experiment suggests that the onset of the OFT defects coincides with the first observable activation of the TGF- $\beta$ pathway in OFT ECs. An excessive expansion of the OFT is often linked to a defective SMC wall. Thus, I analyzed the formation of the SMC wall in alk.5 mutants at $75 \mathrm{hpf}$ by live imaging, immunostainings, and transmission electron microscopy (TEM). I observed that alk $5 \%$ OFTs were surrounded by a reduced number of SMCs compared with wild types. Contrarily to ECs, SMCs appeared less proliferative when Alk5 was absent. alk. $5^{-1}$ OFT walls also exhibited wider extracellular spaces, as shown by TEM, and a compromised ECM environment, as confirmed by impaired Elastin2 immunostaining.

To understand which cell type was responsible for the OFT phenotype, I overexpressed wildtype alk $5 b$ specifically in ECs, injecting the $\mathrm{Tg}(f l i 1$ a:alk.5b-P2A-mScarlet) construct. While the EC-specific overexpression did not cause any defect in wild-type animals, it completely rescued the alk.5 mutant phenotype both morphologically and functionally. Surprisingly, the sole ECrescue not only restored EC proliferation in alk.5 mutants, but also led to a complete rescue of SMC proliferation and ECM composition. These results strongly suggest that Alk5 is sufficient in ECs, from where it orchestrates the intercellular communication with SMCs.

To better characterize molecularly the origin and nature of this interaction, I performed transcriptomic analyses on $56 \mathrm{hpf}$ extracted hearts following Alk5 inhibition. I observed that among the several biological processes and cellular compartments, the ECM was the most severely affected. I identified and further investigated one candidate gene, $f b \ln 5$, encoding an integrin-binding protein, which proved to be a very specific OFT marker in embryonic zebrafish. Notably, fbln 5 EC-specific overexpression in alk.5 mutant could partially restore EC proliferation, Eln2 coverage, and OFT expansion. These data suggest a role of Fbln5 downstream of Alk5 in mediating the EC-SMC cross talk, potentially by promoting EC attachment to the matrix, thereby inhibiting their proliferation (Sullivan et al., 2007; Williamson et al., 2007), and by supporting in vivo elastogenesis (Nakamura et al., 2002). 


\section{Summaries}

Altogether, in this part of the study, I propose a model where Alk5 mediates EC-SMC communication in the cardiac OFT, by regulating its ECM environment.

In the second part of this study, I aimed at investigating the cross-talk between epicardium and myocardium in the zebrafish larval hearts. The epicardium-myocardium interaction and its role in cardiac repair have been extensively studied in adult mice and zebrafish, focusing on its contribution of new cell types and mitogenic factors (Pennisi et al., 2003; Smits et al., 2018; Quijada et al., 2020). Nonetheless, while there is evidence of the role of the epicardium in early cardiac development and its coverage of the heart temporally coincides with important myocardial morphogenetic events, its effect on $\mathrm{CM}$ behavior requires further characterization. Therefore, I aimed to combine live imaging with several models of epicardial coverage impairment to elucidate the distinct roles for this tissue in sustaining cardiac morphogenesis. Among the well-known epicardial markers, we mutated the transcription factor genes $\operatorname{tcf} 21$ and $w t 1 a$. By live imaging of the $T g(t c f 21: n l s-E G F P)$ transgenic line and performing pan-epicardial immunostaining, I observed that both mutations in tcf21 and $w t 1 a$ reduced epicardial coverage of the ventricle. Remarkably, while wt1a mutants exhibit a complete absence of $t c f 21^{+}$cells adhering to the ventricle, $\operatorname{tcf} 21$ mutants ranged from a complete loss to a level similar to wild types. To complement the study, I sought to obtain a temporally-regulated model of impaired epicardial coverage. I specifically ablated $\operatorname{tcf} 21^{+}$epicardial cells using the bacterial enzyme nitroreductase (NTR), which specifically induces apoptosis upon administration of its substrate, Metronidazole (Mtz). The treatment of $\operatorname{Tg}(t c f 21: m C h e r r y-N T R)$ larvae (Nagelberg et al., 2015, Wang et al., 2015) from 54 to 100 hpf phenocopied the absence of epicardial coverage observed in the two genetic models. Altogether, these three partially independent models constitute a fundamental basis to study the effect of lack of epicardium on heart morphogenesis.

I observed that in absence of the epicardium, 96 hpf larvae of all three models exhibited a reduced cardiac size, a defect not observed at earlier stages. Notably, the ventricular volume in wild-type animals increased between 72 and $96 \mathrm{hpf}$, while in tcf 21 and wt1 a mutants it failed to grow over time. The increase in organ size can be driven by hyperplastic and/or hypertrophic growth, if it is directed by an increase in cell number or cell size, respectively. Therefore, to understand the cellular processes affected in absence of epicardium, I first analyzed CM number and proliferation and observed that they were not affected in any of the models. To investigate CM hypertrophic growth, I tracked specific CMs over time in $\operatorname{tcf} 21^{+/+}$and $\operatorname{tcf} 21^{\%}$ hearts mosaically expressing myl7:mScarlet. By measuring single CM volume, I observed that in $t c f 21^{+/+}$ hearts it increased by $25.7 \%$ between 76 and $100 \mathrm{hpf}$, while $\operatorname{tcf} 21 \% \mathrm{CM}$ volume did not change $(+1.4 \%)$. Altogether, my observations propose an early role for the epicardium in promoting hypertrophic growth during ventricular development. 


\section{Summaries}

To further support the dependency of the impaired cardiac growth on the lack of epicardium, I took advantage of the temporal versatility of the NTR/Mtz system. I relied on the ability of the epicardium to reactivate and regenerate after injury (Wang et al., 2015; Cao and Poss, 2018), to understand whether rescuing the epicardial coverage would be sufficient to improve ventricular growth. Notably, with this approach, I observed that epicardial regeneration from 4 to $6 \mathrm{dpf}$ (days post fertilization), was sufficient to significantly ameliorate the ventricular volume.

Next, I aimed at identifying epicardial factors regulating the cross-talk with the myocardium. Therefore, I took advantage of the portion of $t c f 21$ mutants, that have defects in ventricular growth despite a morphologically normal epicardial coverage. The lack of correlation between the ventricular volume and the number of $t c f 21^{+}$attached to the ventricle in these mutants confirmed that the epicardium is not sufficient as a mechanical barrier, but is required to provide factors essential for myocardial growth. Towards understanding the missing epicardial factors necessary for proper myocardial development in $\operatorname{tcf} 21$ mutants, I performed transcriptomic analyses on sorted $\operatorname{tcf} 21^{+/+}$and $t c f 21^{\%}$ epicardial cells and CMs at $96 \mathrm{hpf}$. The wild-type epicardial dataset is strongly enriched for the expected signaling pathways, such as FGF, TGF$\beta$, and PDGF indicating that our dataset reflects the molecular processes involved in epicardium-myocardium communication. Among the gene specifically downregulated in $t c f 21^{-}$

1- hearts, the secreted factors vegfa and $f g 24$, are of particular interest, as both signal through the common ERK pathway. Therefore, I assessed the activation of the ERK pathway, using a novel biosensor transgenic line, in CMs following epicardial ablation, and I observed its downregulation. Moreover, small molecule inhibitors of both VEGF and FGF pathways lead to a reduced ventricular volume in wild-type hearts, suggesting their involvement in processes related to myocardial growth.

Thus, through the second part of my study, I describe a detailed characterization of three novel models of epicardial disruption. My data suggest an unexpected role for the epicardium in regulating CM hypertrophic growth during early cardiac morphogenesis, potentially driving a subsequent defect in cell division, as often described in eukaryotic cells (Jorgensen and Tyers, 2004). In the future, studies in these models will be important to further dissect the molecular role of the VEGF and FGF factors in regulating CM hypertrophic growth, and to identify novel epicardial factors regulating myocardial development and repair.

\section{Conclusions}

In conclusion, I have characterized two important, yet understudied hubs of intercellular crosstalk in the developing heart, identifying unexpected roles for non-muscular tissues.

I first described a critical role for the TGF- $\beta$ signaling in orchestrating the EC-SMC cross-talk in the developing outflow tract, by regulating the extracellular environment. I observed a 


\section{Summaries}

previously unappreciated role for Alk5 in the endothelium during OFT development, which could be extended to the study of vascular pathologies. Moreover, I characterized the complex downstream molecular pathway leading to the OFT defects, providing new insights to explain the controversial results obtained in mammals.

Furthermore, I contributed with new models to the study of the epicardium-myocardium interaction. Analyses of these three models have revealed a new role for the epicardium, through FGF and VEGF, in promoting CM hypertrophic growth. These tools also provide valuable datasets that can be easily extended to identify more pathways. In the future, these studies could serve as a platform to characterize the interdependency between hyperplastic and hypertrophic growth and the role of the epicardium in these processes. 


\section{Bibliography}

Acharya, A., Baek, S. T., Huang, G., Eskiocak, B., Goetsch, S., Sung, C. Y., . . Tallquist, M. D. (2012). The bHLH transcription factor Tcf21 is required for lineage-specific EMT of cardiac fibroblast progenitors. Development, 139(12), 2139-2149. doi:10.1242/dev.079970

Albig, A. R., \& Schiemann, W. P. (2004). Fibulin-5 antagonizes vascular endothelial growth factor (VEGF) signaling and angiogenic sprouting by endothelial cells. DNA Cell Biol, 23(6), 367379. doi:10.1089/104454904323145254

Ando, K., Fukuhara, S., Izumi, N., Nakajima, H., Fukui, H., Kelsh, R. N., \& Mochizuki, N. (2016). Clarification of mural cell coverage of vascular endothelial cells by live imaging of zebrafish. Development, 143(8), 1328-1339. doi:10.1242/dev.132654

Aragon, E., Wang, Q., Zou, Y., Morgani, S. M., Ruiz, L., Kaczmarska, Z., . . Massague, J. (2019). Structural basis for distinct roles of SMAD2 and SMAD3 in FOXH1 pioneer-directed TGFbeta signaling. Genes Dev, 33(21-22), 1506-1524. doi:10.1101/gad.330837.119

Ashburner, M., Ball, C. A., Blake, J. A., Botstein, D., Butler, H., Cherry, J. M., . . Sherlock, G. (2000). Gene ontology: tool for the unification of biology. The Gene Ontology Consortium. Nat Genet, 25(1), 25-29. doi:10.1038/75556

Aspalter, I. M., Gordon, E., Dubrac, A., Ragab, A., Narloch, J., Vizan, P., . . Gerhardt, H. (2015). Alk1 and Alk5 inhibition by Nrp1 controls vascular sprouting downstream of Notch. Nat Commun, 6, 7264. doi:10.1038/ncomms8264

Auman, H. J., Coleman, H., Riley, H. E., Olale, F., Tsai, H. J., \& Yelon, D. (2007). Functional modulation of cardiac form through regionally confined cell shape changes. PLOS Biol, 5(3), e53. doi:10.1371/journal.pbio.0050053

Azhar, M., Runyan, R. B., Gard, C., Sanford, L. P., Miller, M. L., Andringa, A., .. . Doetschman, T. (2009). Ligand-specific function of transforming growth factor beta in epithelialmesenchymal transition in heart development. Dev Dyn, 238(2), 431-442. doi:10.1002/dvdy.21854

Azhar, M., Brown, K., Gard, C., Chen, H., Rajan, S., Elliott, D. A., .. . Doetschman, T. (2011). Transforming growth factor Beta2 is required for valve remodeling during heart development. Dev Dyn, 240(9), 2127-2141. doi:10.1002/dvdy.22702

Bajolle, F., Zaffran, S., Kelly, R. G., Hadchouel, J., Bonnet, D., Brown, N. A., \& Buckingham, M. E. (2006). Rotation of the myocardial wall of the outflow tract is implicated in the normal positioning of the great arteries. Circ Res, 98(3), 421-428. doi:10.1161/01.RES.0000202800.85341.6e

Baldwin, H. S. (1996). Early embryonic vascular development. Cardiovasc Res, 31 Spec No, E34-45.

Beis, D., Bartman, T., Jin, S. W., Scott, I. C., D'Amico, L. A., Ober, E. A., . . Jungblut, B. (2005). Genetic and cellular analyses of zebrafish atrioventricular cushion and valve development. Development, 132(18), 4193-4204. doi:10.1242/dev.01970

Bensimon-Brito, A., Ramkumar, S., Boezio, G. L. M., Guenther, S., Kuenne, C., Helker, C. S. M., . . . Stainier, D. Y. R. (2020). TGF-beta Signaling Promotes Tissue Formation during Cardiac Valve Regeneration in Adult Zebrafish. Dev Cell, 52(1), 9-20 e27. doi:10.1016/j.devcel.2019.10.027

Bergmann, O., Zdunek, S., Felker, A., Salehpour, M., Alkass, K., Bernard, S., . . Frisen, J. (2015). Dynamics of Cell Generation and Turnover in the Human Heart. Cell, 161(7), 1566-1575. doi:10.1016/j.cell.2015.05.026

Boezio, G. L., Bensimon-Brito, A., Piesker, J., Guenther, S., Helker, C. S., \& Stainier, D. Y. (2020). Endothelial TGF-beta signaling instructs smooth muscle cell development in the cardiac outflow tract. Elife, 9. doi:10.7554/eLife.57603

Brade, T., Kumar, S., Cunningham, T. J., Chatzi, C., Zhao, X., Cavallero, S., . . Duester, G. (2011). Retinoic acid stimulates myocardial expansion by induction of hepatic erythropoietin which activates epicardial Igf2. Development, 138(1), 139-148. doi:10.1242/dev.054239 
Braitsch, C. M., Combs, M. D., Quaggin, S. E., \& Yutzey, K. E. (2012). Pod1/Tcf21 is regulated by retinoic acid signaling and inhibits differentiation of epicardium-derived cells into smooth muscle in the developing heart. Developmental Biology, 368(2), 345-357. doi:10.1016/j.ydbio.2012.06.002

Bressan, M., Yang, P. B., Louie, J. D., Navetta, A. M., Garriock, R. J., \& Mikawa, T. (2014). Reciprocal myocardial-endocardial interactions pattern the delay in atrioventricular junction conduction. Development, 141(21), 4149-4157. doi:10.1242/dev.110007

Brown, D. R., Samsa, L. A., Qian, L., \& Liu, J. (2016). Advances in the Study of Heart Development and Disease Using Zebrafish. J Cardiovasc Dev Dis, 3(2). doi:10.3390/jcdd3020013

Brown, K. A., Pietenpol, J. A., \& Moses, H. L. (2007). A tale of two proteins: differential roles and regulation of Smad2 and Smad3 in TGF-beta signaling. J Cell Biochem, 101(1), 9-33. doi:10.1002/jcb.21255

Brownstein, A. J., Ziganshin, B. A., Kuivaniemi, H., Body, S. C., Bale, A. E., \& Elefteriades, J. A. (2017). Genes Associated with Thoracic Aortic Aneurysm and Dissection: An Update and Clinical Implications. Aorta (Stamford), 5(1), 11-20. doi:10.12945/j.aorta.2017.17.003

Bruneau, B. G. (2013). Signaling and transcriptional networks in heart development and regeneration. Cold Spring Harb Perspect Biol, 5(3), a008292. doi:10.1101/cshperspect.a008292

Buckingham, M., Meilhac, S., \& Zaffran, S. (2005). Building the mammalian heart from two sources of myocardial cells. Nat Rev Genet, 6(11), 826-835. doi:10.1038/nrg1710

Burg, L., Palmer, N., Kikhi, K., Miroshnik, E. S., Rueckert, H., Gaddy, E., . . Balciunas, D. (2018). Conditional mutagenesis by oligonucleotide-mediated integration of loxP sites in zebrafish. PLoS Genet, 14(11), e1007754. doi:10.1371/journal.pgen.1007754

Bussmann, J., \& Schulte-Merker, S. (2011). Rapid BAC selection for tol2-mediated transgenesis in zebrafish. Development, 138(19), 4327-4332. doi:10.1242/dev.068080

Camerota, L., Ritelli, M., Wischmeijer, A., Majore, S., Cinquina, V., Fortugno, P., . . Brancati, F. (2019). Genotypic Categorization of Loeys-Dietz Syndrome Based on 24 Novel Families and Literature Data. Genes (Basel), 10(10). doi:10.3390/genes10100764

Cao, J., Navis, A., Cox, B. D., Dickson, A. L., Gemberling, M., Karra, R., . . Poss, K. D. (2016). Single epicardial cell transcriptome sequencing identifies Caveolin 1 as an essential factor in zebrafish heart regeneration. Development, 143(2), 232-243. doi:10.1242/dev.130534

Cao, J., \& Poss, K. D. (2018). The epicardium as a hub for heart regeneration. Nat Rev Cardiol, 15(10), 631-647. doi:10.1038/s41569-018-0046-4

Carvalho, R. L., Itoh, F., Goumans, M. J., Lebrin, F., Kato, M., Takahashi, S., . . Mummery, C. L. (2007). Compensatory signalling induced in the yolk sac vasculature by deletion of TGFbeta receptors in mice. J Cell Sci, 120(Pt 24), 4269-4277. doi:10.1242/jcs.013169

Casari, A., Schiavone, M., Facchinello, N., Vettori, A., Meyer, D., Tiso, N., . . Argenton, F. (2014). A Smad3 transgenic reporter reveals TGF-beta control of zebrafish spinal cord development. Developmental Biology, 396(1), 81-93. doi:10.1016/j.ydbio.2014.09.025

Cavanaugh, A. M., Huang, J., \& Chen, J. N. (2015). Two developmentally distinct populations of neural crest cells contribute to the zebrafish heart. Developmental Biology, 404(2), 103112. doi:10.1016/j.ydbio.2015.06.002

Chablais, F., \& Jazwinska, A. (2012). The regenerative capacity of the zebrafish heart is dependent on TGFbeta signaling. Development, 139(11), 1921-1930. doi:10.1242/dev.078543

Chapman, S. L., Sicot, F. X., Davis, E. C., Huang, J., Sasaki, T., Chu, M. L., \& Yanagisawa, H. (2010). Fibulin-2 and fibulin- 5 cooperatively function to form the internal elastic lamina and protect from vascular injury. Arterioscler Thromb Vasc Biol, 30(1), 68-74. doi:10.1161/ATVBAHA.109.196725

Chen, S., Kulik, M., \& Lechleider, R. J. (2003). Smad proteins regulate transcriptional induction of the SM22alpha gene by TGF-beta. Nucleic Acids Res, 31(4), 1302-1310. doi:10.1093/nar/gkg224 
Chen, T., Chang, T. C., Kang, J. O., Choudhary, B., Makita, T., Tran, C. M., .. Sucov, H. M. (2002). Epicardial induction of fetal cardiomyocyte proliferation via a retinoic acid-inducible trophic factor. Developmental Biology, 250(1), 198-207. doi:10.1006/dbio.2002.0796

Choudhary, B., Zhou, J., Li, P., Thomas, S., Kaartinen, V., \& Sucov, H. M. (2009). Absence of TGFbeta signaling in embryonic vascular smooth muscle leads to reduced lysyl oxidase expression, impaired elastogenesis, and aneurysm. Genesis, 47(2), 115-121. doi:10.1002/dvg.20466

Clift, P. F., \& Cervi, E. (2020). A review of thoracic aortic aneurysm disease. Echo Res Pract, 7(1), R1-R10. doi:10.1530/ERP-19-0049

Colliva, A., Braga, L., Giacca, M., \& Zacchigna, S. (2020). Endothelial cell-cardiomyocyte crosstalk in heart development and disease. J Physiol, 598(14), 2923-2939. doi:10.1113/JP276758

Cunha, S. I., Pardali, E., Thorikay, M., Anderberg, C., Hawinkels, L., Goumans, M. J., . . P Pietras, K. (2010). Genetic and pharmacological targeting of activin receptor-like kinase 1 impairs tumor growth and angiogenesis. J Exp Med, 207(1), 85-100. doi:10.1084/jem.20091309

Cuttano, R., Rudini, N., Bravi, L., Corada, M., Giampietro, C., Papa, E., ... Dejana, E. (2016). KLF4 is a key determinant in the development and progression of cerebral cavernous malformations. EMBO Mol Med, 8(1), 6-24. doi:10.15252/emmm.201505433

D'Amico, L., Scott, I. C., Jungblut, B., \& Stainier, D. Y. (2007). A mutation in zebrafish hmgcr1b reveals a role for isoprenoids in vertebrate heart-tube formation. Curr Biol, 17(3), 252259. doi:10.1016/j.cub.2006.12.023

Darrigrand, J. F., Valente, M., Comai, G., Martinez, P., Petit, M., Nishinakamura, R., . . Cadot, B. (2020). Dullard-mediated Smad1/5/8 inhibition controls mouse cardiac neural crest cells condensation and outflow tract septation. Elife, 9. doi:10.7554/eLife.50325

Dave, J. M., Mirabella, T., Weatherbee, S. D., \& Greif, D. M. (2018). Pericyte ALK5/TIMP3 Axis Contributes to Endothelial Morphogenesis in the Developing Brain. Dev Cell, 44(6), 665678 e666. doi:10.1016/j.devcel.2018.01.018

Davis, G. E., \& Senger, D. R. (2005). Endothelial extracellular matrix: biosynthesis, remodeling, and functions during vascular morphogenesis and neovessel stabilization. Circ Res, 97(11), 1093-1107. doi:10.1161/01.RES.0000191547.64391.e3

Davis, M. P., van Dongen, S., Abreu-Goodger, C., Bartonicek, N., \& Enright, A. J. (2013). Kraken: a set of tools for quality control and analysis of high-throughput sequence data. Methods, 63(1), 41-49. doi:10.1016/j.ymeth.2013.06.027

de Boer, B. A., van den Berg, G., de Boer, P. A., Moorman, A. F., \& Ruijter, J. M. (2012). Growth of the developing mouse heart: an interactive qualitative and quantitative $3 \mathrm{D}$ atlas. Developmental Biology, 368(2), 203-213. doi:10.1016/j.ydbio.2012.05.001

de la Cova, C., Townley, R., Regot, S., \& Greenwald, I. (2017). A Real-Time Biosensor for ERK Activity Reveals Signaling Dynamics during C. elegans Cell Fate Specification. Dev Cell, 42(5), 542-553 e544. doi:10.1016/j.devcel.2017.07.014

De Val, S. (2011). Key transcriptional regulators of early vascular development. Arterioscler Thromb Vasc Biol, 31(7), 1469-1475. doi:10.1161/ATVBAHA.110.221168

Derrick, C. J., \& Noel, E. S. (2021). The ECM as a driver of heart development and repair. Development, 148(5). doi:10.1242/dev.191320

Derynck, R., \& Budi, E. H. (2019). Specificity, versatility, and control of TGF-beta family signaling. Sci Signal, 12(570). doi:10.1126/scisignal.aav5183

Desgrange, A., Le Garrec, J. F., \& Meilhac, S. M. (2018). Left-right asymmetry in heart development and disease: forming the right loop. Development, 145(22). doi:10.1242/dev.162776

Dey, K. K., Hsiao, C. J., \& Stephens, M. (2017). Visualizing the structure of RNA-seq expression data using grade of membership models. PLoS Genet, 13(3), e1006599. doi:10.1371/journal.pgen.1006599 
Dobin, A., Davis, C. A., Schlesinger, F., Drenkow, J., Zaleski, C., Jha, S., . . Gingeras, T. R. (2013). STAR: ultrafast universal RNA-seq aligner. Bioinformatics, 29(1), 15-21. doi:10.1093/bioinformatics/bts635

Dogra, D., Ahuja, S., Kim, H. T., Rasouli, S. J., Stainier, D. Y. R., \& Reischauer, S. (2017). Opposite effects of Activin type 2 receptor ligands on cardiomyocyte proliferation during development and repair. Nat Commun, 8(1), 1902. doi:10.1038/s41467-017-01950-1

Duchemin, A. L., Vignes, H., \& Vermot, J. (2019). Mechanically activated piezo channels modulate outflow tract valve development through the Yap1 and KIf2-Notch signaling axis. Elife, 8. doi:10.7554/eLife.44706

Eid, H., Larson, D. M., Springhorn, J. P., Attawia, M. A., Nayak, R. C., Smith, T. W., \& Kelly, R. A. (1992). Role of epicardial mesothelial cells in the modification of phenotype and function of adult rat ventricular myocytes in primary coculture. Circ Res, 71(1), 40-50. doi:10.1161/01.res.71.1.40

El-Brolosy, M. A., Kontarakis, Z., Rossi, A., Kuenne, C., Gunther, S., Fukuda, N., . . Stainier, D. Y. R. (2019). Genetic compensation triggered by mutant mRNA degradation. Nature, 568(7751), 193-197. doi:10.1038/s41586-019-1064-z

Fabregat, A., Korninger, F., Viteri, G., Sidiropoulos, K., Marin-Garcia, P., Ping, P., . . Hermjakob, H. (2018). Reactome graph database: Efficient access to complex pathway data. PLoS Comput Biol, 14(1), e1005968. doi:10.1371/journal.pcbi.1005968

Fadini, G. P., \& Tjwa, M. (2010). A role for TGF-beta in transforming endothelial progenitor cells into neointimal smooth muscle cells. Atherosclerosis, 211(1), 32-35. doi:10.1016/j.atherosclerosis.2010.03.025

Felker, A., Prummel, K. D., Merks, A. M., Mickoleit, M., Brombacher, E. C., Huisken, J., . . Mosimann, C. (2018). Continuous addition of progenitors forms the cardiac ventricle in zebrafish. Nat Commun, 9(1), 2001. doi:10.1038/s41467-018-04402-6

Feng, X. H., \& Derynck, R. (2005). Specificity and versatility in tgf-beta signaling through Smads. Annu Rev Cell Dev Biol, 21, 659-693. doi:10.1146/annurev.cellbio.21.022404.142018

Fountoulaki, K., Dagres, N., \& Iliodromitis, E. K. (2015). Cellular Communications in the Heart. Card Fail Rev, 1(2), 64-68. doi:10.15420/cfr.2015.1.2.64

Gaengel, K., Genove, G., Armulik, A., \& Betsholtz, C. (2009). Endothelial-mural cell signaling in vascular development and angiogenesis. Arterioscler Thromb Vasc Biol, 29(5), 630-638. doi:10.1161/ATVBAHA.107.161521

Gagnon, J. A., Valen, E., Thyme, S. B., Huang, P., Akhmetova, L., Pauli, A., . . Schier, A. F. (2014). Efficient mutagenesis by Cas9 protein-mediated oligonucleotide insertion and large-scale assessment of single-guide RNAs. PLoS One, 9(5), e98186. doi:10.1371/journal.pone.0098186

Gellibert, F., Woolven, J., Fouchet, M. H., Mathews, N., Goodland, H., Lovegrove, V., . . Hartley, D. (2004). Identification of 1,5-naphthyridine derivatives as a novel series of potent and selective TGF-beta type I receptor inhibitors. J Med Chem, 47(18), 4494-4506. doi:10.1021/jm0400247

Gene Ontology, C. (2021). The Gene Ontology resource: enriching a GOld mine. Nucleic Acids Res, 49(D1), D325-D334. doi:10.1093/nar/gkaa1113

Gentile, A., Bensimon-Brito, A., Priya, R., Maischein, H.-M., Piesker, J., Günther, S., . . Stainier, D. Y. R. (2020). The EMT transcription factor Snai1 maintains myocardial wall integrity by repressing intermediate filament gene expression. bioRxiv, 2020.2012.2015.422833. doi:10.1101/2020.12.15.422833

Gillis, E., Van Laer, L., \& Loeys, B. L. (2013). Genetics of thoracic aortic aneurysm: at the crossroad of transforming growth factor-beta signaling and vascular smooth muscle cell contractility. Circ Res, 113(3), 327-340. doi:10.1161/CIRCRESAHA.113.300675

Gittenberger-de Groot, A. C., Vrancken Peeters, M. P., Bergwerff, M., Mentink, M. M., \& Poelmann, R. E. (2000). Epicardial outgrowth inhibition leads to compensatory 
mesothelial outflow tract collar and abnormal cardiac septation and coronary formation. Circ Res, 87(11), 969-971. doi:10.1161/01.res.87.11.969

Goujon, M., McWilliam, H., Li, W., Valentin, F., Squizzato, S., Paern, J., \& Lopez, R. (2010). A new bioinformatics analysis tools framework at EMBL-EBI. Nucleic Acids Res, 38(Web Server issue), W695-699. doi:10.1093/nar/gkq313

Goumans, M. J., Valdimarsdottir, G., Itoh, S., Rosendahl, A., Sideras, P., \& ten Dijke, P. (2002). Balancing the activation state of the endothelium via two distinct TGF-beta type I receptors. EMBO J, 21(7), 1743-1753. doi:10.1093/emboj/21.7.1743

Goumans, M. J., Lebrin, F., \& Valdimarsdottir, G. (2003). Controlling the angiogenic switch: a balance between two distinct TGF-b receptor signaling pathways. Trends Cardiovasc Med, 13(7), 301-307. doi:10.1016/s1050-1738(03)00142-7

Goumans, M. J., Valdimarsdottir, G., Itoh, S., Lebrin, F., Larsson, J., Mummery, C., . . . ten Dijke, P. (2003). Activin receptor-like kinase (ALK) 1 is an antagonistic mediator of lateral TGFbeta/ALK5 signaling. Mol Cell, 12(4), 817-828. doi:10.1016/s1097-2765(03)00386-1

Goumans, M. J., van Zonneveld, A. J., \& ten Dijke, P. (2008). Transforming growth factor betainduced endothelial-to-mesenchymal transition: a switch to cardiac fibrosis? Trends Cardiovasc Med, 18(8), 293-298. doi:10.1016/j.tcm.2009.01.001

Goumans, M. J., \& Ten Dijke, P. (2018). TGF-beta Signaling in Control of Cardiovascular Function. Cold Spring Harb Perspect Biol, 10(2). doi:10.1101/cshperspect.a022210

Grego-Bessa, J., Luna-Zurita, L., del Monte, G., Bolos, V., Melgar, P., Arandilla, A., . . . de la Pompa, J. L. (2007). Notch signaling is essential for ventricular chamber development. Dev Cell, 12(3), 415-429. doi:10.1016/j.devcel.2006.12.011

Grimes, A. C., Stadt, H. A., Shepherd, I. T., \& Kirby, M. L. (2006). Solving an enigma: arterial pole development in the zebrafish heart. Developmental Biology, 290(2), 265-276. doi:10.1016/j.ydbio.2005.11.042

Grimes, A. C., \& Kirby, M. L. (2009). The outflow tract of the heart in fishes: anatomy, genes and evolution. J Fish Biol, 74(5), 983-1036. doi:10.1111/j.1095-8649.2008.02125.x

Grimes, A. C., Duran, A. C., Sans-Coma, V., Hami, D., Santoro, M. M., \& Torres, M. (2010). Phylogeny informs ontogeny: a proposed common theme in the arterial pole of the vertebrate heart. Evol Dev, 12(6), 552-567. doi:10.1111/j.1525-142X.2010.00441.x

Gronroos, E., Kingston, I. J., Ramachandran, A., Randall, R. A., Vizan, P., \& Hill, C. S. (2012). Transforming growth factor beta inhibits bone morphogenetic protein-induced transcription through novel phosphorylated Smad1/5-Smad3 complexes. Mol Cell Biol, 32(14), 2904-2916. doi:10.1128/MCB.00231-12

Guerra, A., Germano, R. F., Stone, O., Arnaout, R., Guenther, S., Ahuja, S., . . Reischauer, S. (2018). Distinct myocardial lineages break atrial symmetry during cardiogenesis in zebrafish. Elife, 7. doi:10.7554/eLife.32833

Gunawan, F., Gentile, A., Fukuda, R., Tsedeke, A. T., Jimenez-Amilburu, V., Ramadass, R., . . Stainier, D. Y. R. (2019). Focal adhesions are essential to drive zebrafish heart valve morphogenesis. J Cell Biol, 218(3), 1039-1054. doi:10.1083/jcb.201807175

Guner-Ataman, B., Paffett-Lugassy, N., Adams, M. S., Nevis, K. R., Jahangiri, L., Obregon, P., ... Burns, C. G. (2013). Zebrafish second heart field development relies on progenitor specification in anterior lateral plate mesoderm and nkx2.5 function. Development, 140(6), 1353-1363. doi:10.1242/dev.088351

Guo, X., \& Chen, S. Y. (2012). Transforming growth factor-beta and smooth muscle differentiation. World J Biol Chem, 3(3), 41-52. doi:10.4331/wjbc.v3.i3.41

Haack, T., \& Abdelilah-Seyfried, S. (2016). The force within: endocardial development, mechanotransduction and signalling during cardiac morphogenesis. Development, 143(3), 373-386. doi:10.1242/dev.131425

Hagan, A. S., Boylan, M., Smith, C., Perez-Santamarina, E., Kowalska, K., Hung, I. H., . . Ornitz, D. M. (2019). Generation and validation of novel conditional flox and inducible Cre alleles 
targeting fibroblast growth factor 18 (Fgf18). Dev Dyn, 248(9), 882-893.

doi:10.1002/dvdy.85

Hami, D., Grimes, A. C., Tsai, H. J., \& Kirby, M. L. (2011). Zebrafish cardiac development requires a conserved secondary heart field. Development, 138(11), 2389-2398.

doi:10.1242/dev.061473

Han, P., Bloomekatz, J., Ren, J., Zhang, R., Grinstein, J. D., Zhao, L., .. Chi, N. C. (2016). Coordinating cardiomyocyte interactions to direct ventricular chamber morphogenesis. Nature, 534(7609), 700-704. doi:10.1038/nature18310

Harris, I. S., \& Black, B. L. (2010). Development of the endocardium. Pediatr Cardiol, 31(3), 391399. doi:10.1007/s00246-010-9642-8

Hatta, K., Tsujii, H., \& Omura, T. (2006). Cell tracking using a photoconvertible fluorescent protein. Nat Protoc, 1(2), 960-967. doi:10.1038/nprot.2006.96

Hellstrom, M., Kal n, M., Lindahl, P., Abramsson, A., \& Betsholtz, C. (1999). Role of PDGF-B and PDGFR-beta in recruitment of vascular smooth muscle cells and pericytes during embryonic blood vessel formation in the mouse. Development, 126(14), 3047-3055.

Herwig, L., Blum, Y., Krudewig, A., Ellertsdottir, E., Lenard, A., Belting, H. G., \& Affolter, M. (2011). Distinct cellular mechanisms of blood vessel fusion in the zebrafish embryo. Curr Biol, 21(22), 1942-1948. doi:10.1016/j.cub.2011.10.016

Hill, C. S. (2016). Transcriptional Control by the SMADs. Cold Spring Harb Perspect Biol, 8(10). doi:10.1101/cshperspect.a022079

Hirschi, K. K., Rohovsky, S. A., Beck, L. H., Smith, S. R., \& D'Amore, P. A. (1999). Endothelial cells modulate the proliferation of mural cell precursors via platelet-derived growth factor-BB and heterotypic cell contact. Circ Res, 84(3), 298-305. doi:10.1161/01.res.84.3.298

Hishikawa, K., Nakaki, T., \& Fujii, T. (1999). Transforming growth factor-beta(1) induces apoptosis via connective tissue growth factor in human aortic smooth muscle cells. Eur J Pharmacol, 385(2-3), 287-290. doi:10.1016/s0014-2999(99)00763-3

Holtzhausen, A., Golzio, C., How, T., Lee, Y. H., Schiemann, W. P., Katsanis, N., \& Blobe, G. C. (2014). Novel bone morphogenetic protein signaling through Smad2 and Smad3 to regulate cancer progression and development. FASEB J, 28(3), 1248-1267. doi:10.1096/fj.13-239178

Hu, B., Wu, Z., \& Phan, S. H. (2003). Smad3 mediates transforming growth factor-beta-induced alpha-smooth muscle actin expression. Am J Respir Cell Mol Biol, 29(3 Pt 1), 397-404. doi:10.1165/rcmb.2003-00630C

Hutson, M. R., \& Kirby, M. L. (2003). Neural crest and cardiovascular development: a 20-year perspective. Birth Defects Res C Embryo Today, 69(1), 2-13. doi:10.1002/bdrc.10002

Hynes, R. O. (2007). Cell-matrix adhesion in vascular development. J Thromb Haemost, 5 Suppl 1, 32-40. doi:10.1111/j.1538-7836.2007.02569.x

leda, M., Tsuchihashi, T., Ivey, K. N., Ross, R. S., Hong, T. T., Shaw, R. M., \& Srivastava, D. (2009). Cardiac fibroblasts regulate myocardial proliferation through beta1 integrin signaling. Dev Cell, 16(2), 233-244. doi:10.1016/j.devcel.2008.12.007

Ivanovitch, K., Soro-Barrio, P., Chakravarty, P., Jones, R. A., Mousavy Gharavy, S. N., Stamataki, D., ... Briscoe, J. (2020). Ventricular, atrial and outflow tract heart progenitors arise from spatially and molecularly distinct regions of the primitive streak. bioRxiv, 2020.2007.2012.198994. doi:10.1101/2020.07.12.198994

Jaffe, M., Sesti, C., Washington, I. M., Du, L., Dronadula, N., Chin, M. T., . . Dichek, D. A. (2012). Transforming growth factor-beta signaling in myogenic cells regulates vascular morphogenesis, differentiation, and matrix synthesis. Arterioscler Thromb Vasc Biol, 32(1), e1-11. doi:10.1161/ATVBAHA.111.238410

Jahangiri, L., Sharpe, M., Novikov, N., Gonzalez-Rosa, J. M., Borikova, A., Nevis, K., . . Burns, C. G. (2016). The AP-1 transcription factor component Fosl2 potentiates the rate of myocardial differentiation from the zebrafish second heart field. Development, 143(1), 113-122. doi:10.1242/dev.126136 
Jana, S., Hu, M., Shen, M., \& Kassiri, Z. (2019). Extracellular matrix, regional heterogeneity of the aorta, and aortic aneurysm. Exp Mol Med, 51(12), 160. doi:10.1038/s12276-019-0286-3

Jassal, B., Matthews, L., Viteri, G., Gong, C., Lorente, P., Fabregat, A., ... D'Eustachio, P. (2020). The reactome pathway knowledgebase. Nucleic Acids Res, 48(D1), D498-D503. doi:10.1093/nar/gkz1031

Jensen, B., Wang, T., Christoffels, V. M., \& Moorman, A. F. (2013). Evolution and development of the building plan of the vertebrate heart. Biochim Biophys Acta, 1833(4), 783-794. doi:10.1016/j.bbamcr.2012.10.004

Jin, S. W., Beis, D., Mitchell, T., Chen, J. N., \& Stainier, D. Y. (2005). Cellular and molecular analyses of vascular tube and lumen formation in zebrafish. Development, 132(23), 5199-5209. doi:10.1242/dev.02087

Jinnin, M., Ihn, H., \& Tamaki, K. (2006). Characterization of SIS3, a novel specific inhibitor of Smad3, and its effect on transforming growth factor-beta1-induced extracellular matrix expression. Mol Pharmacol, 69(2), 597-607. doi:10.1124/mol.105.017483

Jones, J. A., Spinale, F. G., \& Ikonomidis, J. S. (2009). Transforming growth factor-beta signaling in thoracic aortic aneurysm development: a paradox in pathogenesis. J Vasc Res, 46(2), 119137. doi:10.1159/000151766

Jorgensen, P., \& Tyers, M. (2004). How cells coordinate growth and division. Curr Biol, 14(23), R1014-1027. doi:10.1016/j.cub.2004.11.027

Karra, R., Foglia, M. J., Choi, W. Y., Belliveau, C., DeBenedittis, P., \& Poss, K. D. (2018). Vegfaa instructs cardiac muscle hyperplasia in adult zebrafish. Proc Natl Acad Sci U S A, 115(35), 8805-8810. doi:10.1073/pnas.1722594115

Kastner, P., Grondona, J. M., Mark, M., Gansmuller, A., LeMeur, M., Decimo, D., . . Chambon, P. (1994). Genetic analysis of RXR alpha developmental function: convergence of RXR and RAR signaling pathways in heart and eye morphogenesis. Cell, 78(6), 987-1003. doi:10.1016/0092-8674(94)90274-7

Katz, T. C., Singh, M. K., Degenhardt, K., Rivera-Feliciano, J., Johnson, R. L., Epstein, J. A., \& Tabin, C. J. (2012). Distinct compartments of the proepicardial organ give rise to coronary vascular endothelial cells. Dev Cell, 22(3), 639-650. doi:10.1016/j.devcel.2012.01.012

Keegan, B. R., Meyer, D., \& Yelon, D. (2004). Organization of cardiac chamber progenitors in the zebrafish blastula. Development, 131(13), 3081-3091. doi:10.1242/dev.01185

Kelleher, C. M., McLean, S. E., \& Mecham, R. P. (2004). Vascular extracellular matrix and aortic development. Curr Top Dev Biol, 62, 153-188. doi:10.1016/S0070-2153(04)62006-0

Kelly, R. G., \& Buckingham, M. E. (2002). The anterior heart-forming field: voyage to the arterial pole of the heart. Trends Genet, 18(4), 210-216. doi:10.1016/s0168-9525(02)02642-2

Kelly, R. G., Buckingham, M. E., \& Moorman, A. F. (2014). Heart fields and cardiac morphogenesis. Cold Spring Harb Perspect Med, 4(10). doi:10.1101/cshperspect.a015750

Kikuchi, K., Gupta, V., Wang, J., Holdway, J. E., Wills, A. A., Fang, Y., \& Poss, K. D. (2011). tcf21+ epicardial cells adopt non-myocardial fates during zebrafish heart development and regeneration. Development, 138(14), 2895-2902. doi:10.1242/dev.067041

Kikuchi, K., Holdway, J. E., Major, R. J., Blum, N., Dahn, R. D., Begemann, G., \& Poss, K. D. (2011). Retinoic acid production by endocardium and epicardium is an injury response essential for zebrafish heart regeneration. Dev Cell, 20(3), 397-404. doi:10.1016/j.devcel.2011.01.010

Kim, S. H., Turnbull, J., \& Guimond, S. (2011). Extracellular matrix and cell signalling: the dynamic cooperation of integrin, proteoglycan and growth factor receptor. J Endocrinol, 209(2), 139-151. doi:10.1530/JOE-10-0377

Kimmel, C. B., Ballard, W. W., Kimmel, S. R., Ullmann, B., \& Schilling, T. F. (1995). Stages of embryonic development of the zebrafish. Dev Dyn, 203(3), 253-310. doi:10.1002/aja.1002030302

Kirby, M. L. (2001). Neural Crest and Heart Development. In (pp. 109-120): Birkhäuser Boston. 
Knight, H. G., \& Yelon, D. (2016). Utilizing Zebrafish to Understand Second Heart Field Development. In T. Nakanishi, R. R. Markwald, H. S. Baldwin, B. B. Keller, D. Srivastava, \& $\mathrm{H}$. Yamagishi (Eds.), Etiology and Morphogenesis of Congenital Heart Disease: From Gene Function and Cellular Interaction to Morphology (pp. 193-199). Tokyo.

Kocgozlu, L., Saw, T. B., Le, A. P., Yow, I., Shagirov, M., Wong, E., . . Ladoux, B. (2016). Epithelial Cell Packing Induces Distinct Modes of Cell Extrusions. Curr Biol, 26(21), 2942-2950. doi:10.1016/j.cub.2016.08.057

Kreidberg, J. A., Sariola, H., Loring, J. M., Maeda, M., Pelletier, J., Housman, D., \& Jaenisch, R. (1993). WT-1 is required for early kidney development. Cell, 74(4), 679-691. doi:10.1016/0092-8674(93)90515-r

Kudo, T., Jeknic, S., Macklin, D. N., Akhter, S., Hughey, J. J., Regot, S., \& Covert, M. W. (2018). Livecell measurements of kinase activity in single cells using translocation reporters. Nat Protoc, 13(1), 155-169. doi:10.1038/nprot.2017.128

Kwee, L., Baldwin, H. S., Shen, H. M., Stewart, C. L., Buck, C., Buck, C. A., \& Labow, M. A. (1995). Defective development of the embryonic and extraembryonic circulatory systems in vascular cell adhesion molecule (VCAM-1) deficient mice. Development, 121(2), 489-503.

Lacolley, P., Regnault, V., Segers, P., \& Laurent, S. (2017). Vascular Smooth Muscle Cells and Arterial Stiffening: Relevance in Development, Aging, and Disease. Physiol Rev, 97(4), 1555-1617. doi:10.1152/physrev.00003.2017

Laguens, R., Cabeza Meckert, P., Vera Janavel, G., De Lorenzi, A., Lascano, E., Negroni, J., . . . Crottogini, A. (2004). Cardiomyocyte hyperplasia after plasmid-mediated vascular endothelial growth factor gene transfer in pigs with chronic myocardial ischemia. J Gene Med, 6(2), 222-227. doi:10.1002/jgm.478

Lai, E. C. (2004). Notch signaling: control of cell communication and cell fate. Development, 131(5), 965-973. doi:10.1242/dev.01074

Lamouille, S., Mallet, C., Feige, J. J., \& Bailly, S. (2002). Activin receptor-like kinase 1 is implicated in the maturation phase of angiogenesis. Blood, 100(13), 4495-4501. doi:10.1182/blood.V100.13.4495

Larsson, J., Goumans, M. J., Sjostrand, L. J., van Rooijen, M. A., Ward, D., Leveen, P., . . Karlsson, S. (2001). Abnormal angiogenesis but intact hematopoietic potential in TGF-beta type I receptor-deficient mice. EMBO J, 20(7), 1663-1673. doi:10.1093/emboj/20.7.1663

Lavine, K. J., Yu, K., White, A. C., Zhang, X., Smith, C., Partanen, J., \& Ornitz, D. M. (2005). Endocardial and epicardial derived FGF signals regulate myocardial proliferation and differentiation in vivo. Dev Cell, 8(1), 85-95. doi:10.1016/j.devcel.2004.12.002

Lavine, K. J., White, A. C., Park, C., Smith, C. S., Choi, K., Long, F., ... Ornitz, D. M. (2006). Fibroblast growth factor signals regulate a wave of Hedgehog activation that is essential for coronary vascular development. Genes Dev, 20(12), 1651-1666. doi:10.1101/gad.1411406

Li, M., Qian, M., Kyler, K., \& Xu, J. (2018). Endothelial-Vascular Smooth Muscle Cells Interactions in Atherosclerosis. Front Cardiovasc Med, 5, 151. doi:10.3389/fcvm.2018.00151

Li, P., Cavallero, S., Gu, Y., Chen, T. H., Hughes, J., Hassan, A. B., . . Sucov, H. M. (2011). IGF signaling directs ventricular cardiomyocyte proliferation during embryonic heart development. Development, 138(9), 1795-1805. doi:10.1242/dev.054338

Li, W., Li, Q., Jiao, Y., Qin, L., Ali, R., Zhou, J., . . Tellides, G. (2014). Tgfbr2 disruption in postnatal smooth muscle impairs aortic wall homeostasis. J Clin Invest, 124(2), 755-767. doi:10.1172/JCl69942

Li, Y., Urban, A., Midura, D., Simon, H. G., \& Wang, Q. T. (2017). Proteomic characterization of epicardial-myocardial signaling reveals novel regulatory networks including a role for NFkappaB in epicardial EMT. PLoS One, 12(3), e0174563. doi:10.1371/journal.pone.0174563

Liao, Y., Smyth, G. K., \& Shi, W. (2014). featureCounts: an efficient general purpose program for assigning sequence reads to genomic features. Bioinformatics, 30(7), 923-930. doi:10.1093/bioinformatics/btt656 
Lilly, B. (2014). We have contact: endothelial cell-smooth muscle cell interactions. Physiology (Bethesda), 29(4), 234-241. doi:10.1152/physiol.00047.2013

Lin, F., \& Yang, X. (2010). TGF-beta signaling in aortic aneurysm: another round of controversy. J Genet Genomics, 37(9), 583-591. doi:10.1016/S1673-8527(09)60078-3

Lin, Y. F., Swinburne, I., \& Yelon, D. (2012). Multiple influences of blood flow on cardiomyocyte hypertrophy in the embryonic zebrafish heart. Developmental Biology, 362(2), 242-253. doi:10.1016/j.ydbio.2011.12.005

Lindblom, P., Gerhardt, H., Liebner, S., Abramsson, A., Enge, M., Hellstrom, M., . . Betsholtz, C. (2003). Endothelial PDGF-B retention is required for proper investment of pericytes in the microvessel wall. Genes Dev, 17(15), 1835-1840. doi:10.1101/gad.266803

Liu, J., Bressan, M., Hassel, D., Huisken, J., Staudt, D., Kikuchi, K., . . Stainier, D. Y. (2010). A dual role for ErbB2 signaling in cardiac trabeculation. Development, 137(22), 3867-3875. doi:10.1242/dev.053736

Liu, J., \& Stainier, D. Y. (2010). Tbx5 and Bmp signaling are essential for proepicardium specification in zebrafish. Circ Res, 106(12), 1818-1828. doi:10.1161/CIRCRESAHA.110.217950

Liu, X., Chen, W., Li, W., Li, Y., Priest, J. R., Zhou, B., .. . Zhou, Z. (2019). Single-Cell RNA-Seq of the Developing Cardiac Outflow Tract Reveals Convergent Development of the Vascular Smooth Muscle Cells. Cell Rep, 28(5), 1346-1361 e1344. doi:10.1016/j.celrep.2019.06.092

Loeys, B. L., Chen, J., Neptune, E. R., Judge, D. P., Podowski, M., Holm, T., . . Dietz, H. C. (2005). A syndrome of altered cardiovascular, craniofacial, neurocognitive and skeletal development caused by mutations in TGFBR1 or TGFBR2. Nat Genet, 37(3), 275-281. doi:10.1038/ng1511

Loeys, B. L., Schwarze, U., Holm, T., Callewaert, B. L., Thomas, G. H., Pannu, H., . . Dietz, H. C. (2006). Aneurysm syndromes caused by mutations in the TGF-beta receptor. N Engl J Med, 355(8), 788-798. doi:10.1056/NEJMoa055695

Love, M. I., Huber, W., \& Anders, S. (2014). Moderated estimation of fold change and dispersion for RNA-seq data with DESeq2. Genome Biol, 15(12), 550. doi:10.1186/s13059-014-05508

Lu, S. Y., Sheikh, F., Sheppard, P. C., Fresnoza, A., Duckworth, M. L., Detillieux, K. A., \& Cattini, P. A. (2008). FGF-16 is required for embryonic heart development. Biochem Biophys Res Commun, 373(2), 270-274. doi:10.1016/j.bbrc.2008.06.029

MacGrogan, D., Munch, J., \& de la Pompa, J. L. (2018). Notch and interacting signalling pathways in cardiac development, disease, and regeneration. Nat Rev Cardiol, 15(11), 685-704. doi:10.1038/s41569-018-0100-2

Maddaluno, L., Rudini, N., Cuttano, R., Bravi, L., Giampietro, C., Corada, M., .. . Dejana, E. (2013). EndMT contributes to the onset and progression of cerebral cavernous malformations. Nature, 498(7455), 492-496. doi:10.1038/nature12207

Mahtab, E. A., Vicente-Steijn, R., Hahurij, N. D., Jongbloed, M. R., Wisse, L. J., DeRuiter, M. C., . . Gittenberger-de Groot, A. C. (2009). Podoplanin deficient mice show a RhoA-related hypoplasia of the sinus venosus myocardium including the sinoatrial node. Dev Dyn, 238(1), 183-193. doi:10.1002/dvdy.21819

Mallat, Z., Ait-Oufella, H., \& Tedgui, A. (2017). The Pathogenic Transforming Growth Factor-beta Overdrive Hypothesis in Aortic Aneurysms and Dissections: A Mirage? Circ Res, 120(11), 1718-1720. doi:10.1161/CIRCRESAHA.116.310371

Manasek, F. J. (1969). Embryonic development of the heart. II. Formation of the epicardium. J Embryol Exp Morphol, 22(3), 333-348.

Manner, J. (1993). Experimental study on the formation of the epicardium in chick embryos. Anat Embryol (Berl), 187(3), 281-289. doi:10.1007/BF00195766

Manner, J., Perez-Pomares, J. M., Macias, D., \& Munoz-Chapuli, R. (2001). The origin, formation and developmental significance of the epicardium: a review. Cells Tissues Organs, 169(2), 89-103. doi:10.1159/000047867 
Manner, J., Schlueter, J., \& Brand, T. (2005). Experimental analyses of the function of the proepicardium using a new microsurgical procedure to induce loss-of-proepicardialfunction in chick embryos. Dev Dyn, 233(4), 1454-1463. doi:10.1002/dvdy.20487

Maring, J. A., van Meeteren, L. A., Goumans, M. J., \& Ten Dijke, P. (2016). Interrogating TGF-beta Function and Regulation in Endothelial Cells. Methods Mol Biol, 1344, 193-203. doi:10.1007/978-1-4939-2966-5_11

Martin-Puig, S., Wang, Z., \& Chien, K. R. (2008). Lives of a heart cell: tracing the origins of cardiac progenitors. Cell Stem Cell, 2(4), 320-331. doi:10.1016/j.stem.2008.03.010

Massague, J. (2012). TGFbeta signalling in context. Nat Rev Mol Cell Biol, 13(10), 616-630. doi:10.1038/nrm3434

Masters, M., \& Riley, P. R. (2014). The epicardium signals the way towards heart regeneration. Stem Cell Res, 13(3 Pt B), 683-692. doi:10.1016/j.scr.2014.04.007

Matsuoka, R. L., Marass, M., Avdesh, A., Helker, C. S., Maischein, H. M., Grosse, A. S., . . Stainier, D. Y. (2016). Radial glia regulate vascular patterning around the developing spinal cord. Elife, 5. doi:10.7554/eLife.20253

Matyas, G., Arnold, E., Carrel, T., Baumgartner, D., Boileau, C., Berger, W., \& Steinmann, B. (2006). Identification and in silico analyses of novel TGFBR1 and TGFBR2 mutations in Marfan syndrome-related disorders. Hum Mutat, 27(8), 760-769. doi:10.1002/humu.20353

Meilhac, S. M., Lescroart, F., Blanpain, C., \& Buckingham, M. E. (2014). Cardiac cell lineages that form the heart. Cold Spring Harb Perspect Med, 4(9), a013888. doi:10.1101/cshperspect.a013888

Merki, E., Zamora, M., Raya, A., Kawakami, Y., Wang, J., Zhang, X., . . Ruiz-Lozano, P. (2005). Epicardial retinoid $X$ receptor alpha is required for myocardial growth and coronary artery formation. Proc Natl Acad Sci U S A, 102(51), 18455-18460. doi:10.1073/pnas.0504343102

Miao, M., Bruce, A. E., Bhanji, T., Davis, E. C., \& Keeley, F. W. (2007). Differential expression of two tropoelastin genes in zebrafish. Matrix Biology, 26(2), 115-124. doi:10.1016/j.matbio.2006.09.011

Michel, J. B., Jondeau, G., \& Milewicz, D. M. (2018). From genetics to response to injury: vascular smooth muscle cells in aneurysms and dissections of the ascending aorta. Cardiovasc Res, 114(4), 578-589. doi:10.1093/cvr/cvy006

Milgrom-Hoffman, M., Harrelson, Z., Ferrara, N., Zelzer, E., Evans, S. M., \& Tzahor, E. (2011). The heart endocardium is derived from vascular endothelial progenitors. Development, 138(21), 4777-4787. doi:10.1242/dev.061192

Milgrom-Hoffman, M., Michailovici, I., Ferrara, N., Zelzer, E., \& Tzahor, E. (2014). Endothelial cells regulate neural crest and second heart field morphogenesis. Biol Open, 3(8), 679-688. doi:10.1242/bio.20148078

Misfeldt, A. M., Boyle, S. C., Tompkins, K. L., Bautch, V. L., Labosky, P. A., \& Baldwin, H. S. (2009). Endocardial cells are a distinct endothelial lineage derived from Flk1+ multipotent cardiovascular progenitors. Developmental Biology, 333(1), 78-89. doi:10.1016/j.ydbio.2009.06.033

Mjaatvedt, C. H., Nakaoka, T., Moreno-Rodriguez, R., Norris, R. A., Kern, M. J., Eisenberg, C. A., . . Markwald, R. R. (2001). The outflow tract of the heart is recruited from a novel heartforming field. Developmental Biology, 238(1), 97-109. doi:10.1006/dbio.2001.0409

Moore, A. W., Mclnnes, L., Kreidberg, J., Hastie, N. D., \& Schedl, A. (1999). YAC complementation shows a requirement for $\mathrm{Wt} 1$ in the development of epicardium, adrenal gland and throughout nephrogenesis. Development, 126(9), 1845-1857.

Moorman, A. F., \& Christoffels, V. M. (2003). Cardiac chamber formation: development, genes, and evolution. Physiol Rev, 83(4), 1223-1267. doi:10.1152/physrev.00006.2003

Nagelberg, D., Wang, J., Su, R., Torres-Vazquez, J., Targoff, K. L., Poss, K. D., \& Knaut, H. (2015). Origin, Specification, and Plasticity of the Great Vessels of the Heart. Curr Biol, 25(16), 2099-2110. doi:10.1016/j.cub.2015.06.076 
Nakamura, T., Ruiz-Lozano, P., Lindner, V., Yabe, D., Taniwaki, M., Furukawa, Y., . . Honjo, T. (1999). DANCE, a novel secreted RGD protein expressed in developing, atherosclerotic, and balloon-injured arteries. J Biol Chem, 274(32), 22476-22483.

doi:10.1074/jbc.274.32.22476

Nakamura, T., Lozano, P. R., Ikeda, Y., Iwanaga, Y., Hinek, A., Minamisawa, S., . . Chien, K. R. (2002). Fibulin-5/DANCE is essential for elastogenesis in vivo. Nature, 415(6868), 171-175. doi:10.1038/415171a

Nauroy, P., Hughes, S., Naba, A., \& Ruggiero, F. (2018). The in-silico zebrafish matrisome: A new tool to study extracellular matrix gene and protein functions. Matrix Biol, 65, 5-13. doi:10.1016/j.matbio.2017.07.001

Neeb, Z., Lajiness, J. D., Bolanis, E., \& Conway, S. J. (2013). Cardiac outflow tract anomalies. Wiley Interdiscip Rev Dev Biol, 2(4), 499-530. doi:10.1002/wdev.98

Nicoli, S., Standley, C., Walker, P., Hurlstone, A., Fogarty, K. E., \& Lawson, N. D. (2010). MicroRNAmediated integration of haemodynamics and Vegf signalling during angiogenesis. Nature, 464(7292), 1196-1200. doi:10.1038/nature08889

Oliveros J. C., Venny. An interactive tool for comparing lists with Venn's diagrams. https://bioinfogp.cnb.csic.es/tools/venny/index.html

Olivey, H. E., \& Svensson, E. C. (2010). Epicardial-myocardial signaling directing coronary vasculogenesis. Circ Res, 106(5), 818-832. doi:10.1161/CIRCRESAHA.109.209197

Owens, G. K. (1995). Regulation of differentiation of vascular smooth muscle cells. Physiol Rev, 75(3), 487-517. doi:10.1152/physrev.1995.75.3.487

Owens, G. K., Kumar, M. S., \& Wamhoff, B. R. (2004). Molecular regulation of vascular smooth muscle cell differentiation in development and disease. Physiol Rev, 84(3), 767-801. doi:10.1152/physrev.00041.2003

Paffett-Lugassy, N., Novikov, N., Jeffrey, S., Abrial, M., Guner-Ataman, B., Sakthivel, S., . . Burns, C. G. (2017). Unique developmental trajectories and genetic regulation of ventricular and outflow tract progenitors in the zebrafish second heart field. Development, 144(24), 4616-4624. doi:10.1242/dev.153411

Pardali, E., Goumans, M. J., \& ten Dijke, P. (2010). Signaling by members of the TGF-beta family in vascular morphogenesis and disease. Trends Cell Biol, 20(9), 556-567. doi:10.1016/j.tcb.2010.06.006

Pardali, E., \& Ten Dijke, P. (2012). TGFbeta signaling and cardiovascular diseases. Int J Biol Sci, 8(2), 195-213. doi:10.7150/ijbs.3805

Pennisi, D. J., Ballard, V. L., \& Mikawa, T. (2003). Epicardium is required for the full rate of myocyte proliferation and levels of expression of myocyte mitogenic factors FGF2 and its receptor, FGFR-1, but not for transmural myocardial patterning in the embryonic chick heart. Dev Dyn, 228(2), 161-172. doi:10.1002/dvdy.10360

Peralta, M., Steed, E., Harlepp, S., Gonzalez-Rosa, J. M., Monduc, F., Ariza-Cosano, A., . . . Mercader, N. (2013). Heartbeat-driven pericardiac fluid forces contribute to epicardium morphogenesis. Curr Biol, 23(18), 1726-1735. doi:10.1016/j.cub.2013.07.005

Peralta, M., Gonzalez-Rosa, J. M., Marques, I. J., \& Mercader, N. (2014). The Epicardium in the Embryonic and Adult Zebrafish. J Dev Biol, 2(2), 101-116. doi:10.3390/jdb2020101

Perbellini, F., Watson, S. A., Bardi, I., \& Terracciano, C. M. (2018). Heterocellularity and Cellular Cross-Talk in the Cardiovascular System. Front Cardiovasc Med, 5, 143. doi:10.3389/fcvm.2018.00143

Perez-Pomares, J. M., Phelps, A., Sedmerova, M., Carmona, R., Gonzalez-Iriarte, M., MunozChapuli, R., \& Wessels, A. (2002). Experimental studies on the spatiotemporal expression of WT1 and RALDH2 in the embryonic avian heart: a model for the regulation of myocardial and valvuloseptal development by epicardially derived cells (EPDCs). Developmental Biology, 247(2), 307-326. doi:10.1006/dbio.2002.0706

Perez-Pomares, J. M., Phelps, A., Sedmerova, M., \& Wessels, A. (2003). Epicardial-like cells on the distal arterial end of the cardiac outflow tract do not derive from the proepicardium but 
are derivatives of the cephalic pericardium. Dev Dyn, 227(1), 56-68.

doi:10.1002/dvdy.10284

Perrucci, G. L., Rurali, E., Gowran, A., Pini, A., Antona, C., Chiesa, R., . . Nigro, P. (2017). Vascular smooth muscle cells in Marfan syndrome aneurysm: the broken bricks in the aortic wall. Cell Mol Life Sci, 74(2), 267-277. doi:10.1007/s00018-016-2324-9

Phanish, M. K., Wahab, N. A., Colville-Nash, P., Hendry, B. M., \& Dockrell, M. E. (2006). The differential role of Smad2 and Smad3 in the regulation of pro-fibrotic TGFbeta1 responses in human proximal-tubule epithelial cells. Biochem J, 393(Pt 2), 601-607. doi:10.1042/BJ20051106

Plavicki, J. S., Hofsteen, P., Yue, M. S., Lanham, K. A., Peterson, R. E., \& Heideman, W. (2014). Multiple modes of proepicardial cell migration require heartbeat. BMC Dev Biol, 14, 18. doi:10.1186/1471-213X-14-18

Plein, A., Fantin, A., \& Ruhrberg, C. (2015). Neural crest cells in cardiovascular development. Curr Top Dev Biol, 111, 183-200. doi:10.1016/bs.ctdb.2014.11.006

Pombal, M. A., Carmona, R., Megias, M., Ruiz, A., Perez-Pomares, J. M., \& Munoz-Chapuli, R. (2008). Epicardial development in lamprey supports an evolutionary origin of the vertebrate epicardium from an ancestral pronephric external glomerulus. Evol Dev, 10(2), 210-216. doi:10.1111/j.1525-142X.2008.00228.x

Preis, M., Cohen, T., Sarnatzki, Y., Ben Yosef, Y., Schneiderman, J., Gluzman, Z., . . Flugelman, M. Y. (2006). Effects of fibulin-5 on attachment, adhesion, and proliferation of primary human endothelial cells. Biochem Biophys Res Commun, 348(3), 1024-1033. doi:10.1016/j.bbrc.2006.07.156

Priya, R., Allanki, S., Gentile, A., Mansingh, S., Uribe, V., Maischein, H. M., \& Stainier, D. Y. R. (2020). Tension heterogeneity directs form and fate to pattern the myocardial wall. Nature, 588(7836), 130-134. doi:10.1038/s41586-020-2946-9

Quijada, P., Trembley, M. A., \& Small, E. M. (2020). The Role of the Epicardium During Heart Development and Repair. Circ Res, 126(3), 377-394. doi:10.1161/CIRCRESAHA.119.315857

Raines, E. W. (2000). The extracellular matrix can regulate vascular cell migration, proliferation, and survival: relationships to vascular disease. Int J Exp Pathol, 81(3), 173-182. doi:10.1046/j.1365-2613.2000.00155.x

Rasouli, S. J., \& Stainier, D. Y. R. (2017). Regulation of cardiomyocyte behavior in zebrafish trabeculation by Neuregulin 2a signaling. Nat Commun, 8, 15281. doi:10.1038/ncomms15281

Rasouli, S. J., El-Brolosy, M., Tsedeke, A. T., Bensimon-Brito, A., Ghanbari, P., Maischein, H. M., ... Stainier, D. Y. (2018). The flow responsive transcription factor KIf2 is required for myocardial wall integrity by modulating Fgf signaling. Elife, 7. doi:10.7554/eLife.38889

Renard, M., Callewaert, B., Baetens, M., Campens, L., MacDermot, K., Fryns, J. P., . . De Backer, J. (2013). Novel MYH11 and ACTA2 mutations reveal a role for enhanced TGFbeta signaling in FTAAD. Int J Cardiol, 165(2), 314-321. doi:10.1016/j.ijcard.2011.08.079

Richardson, K. C., Jarett, L., \& Finke, E. H. (1960). Embedding in epoxy resins for ultrathin sectioning in electron microscopy. Stain Technology, 35(6), 313-323. doi:10.3109/10520296009114754

Rochon, E. R., Menon, P. G., \& Roman, B. L. (2016). Alk1 controls arterial endothelial cell migration in lumenized vessels. Development, 143(14), 2593-2602. doi:10.1242/dev.135392

Rothenberg, F., Fisher, S. A., \& Watanabe, M. (2003). Sculpting the cardiac outflow tract. Birth Defects Res C Embryo Today, 69(1), 38-45. doi:10.1002/bdrc.10007

Rottbauer, W., Saurin, A. J., Lickert, H., Shen, X., Burns, C. G., Wo, Z. G., . . Fishman, M. (2002). Reptin and pontin antagonistically regulate heart growth in zebrafish embryos. Cell, 111(5), 661-672. doi:10.1016/s0092-8674(02)01112-1

Runyan, C. E., Schnaper, H. W., \& Poncelet, A. C. (2004). The phosphatidylinositol 3-kinase/Akt pathway enhances Smad3-stimulated mesangial cell collagen I expression in response to 
transforming growth factor-beta1. J Biol Chem, 279(4), 2632-2639.

doi:10.1074/jbc.M310412200

Salabei, J. K., Cummins, T. D., Singh, M., Jones, S. P., Bhatnagar, A., \& Hill, B. G. (2013). PDGFmediated autophagy regulates vascular smooth muscle cell phenotype and resistance to oxidative stress. Biochem J, 451(3), 375-388. doi:10.1042/BJ20121344

Salmon, M., Gomez, D., Greene, E., Shankman, L., \& Owens, G. K. (2012). Cooperative binding of KLF4, pELK-1, and HDAC2 to a G/C repressor element in the SM22alpha promoter mediates transcriptional silencing during SMC phenotypic switching in vivo. Circ Res, 111(6), 685-696. doi:10.1161/CIRCRESAHA.112.269811

Santoro, M. M., Pesce, G., \& Stainier, D. Y. (2009). Characterization of vascular mural cells during zebrafish development. Mech Dev, 126(8-9), 638-649. doi:10.1016/j.mod.2009.06.1080

Sapir, L., \& Tzlil, S. (2017). Talking over the extracellular matrix: How do cells communicate mechanically? Semin Cell Dev Biol, 71, 99-105. doi:10.1016/j.semcdb.2017.06.010

Scherz, P. J., Huisken, J., Sahai-Hernandez, P., \& Stainier, D. Y. (2008). High-speed imaging of developing heart valves reveals interplay of morphogenesis and function. Development, 135(6), 1179-1187. doi:10.1242/dev.010694

Schiemann, W. P., Blobe, G. C., Kalume, D. E., Pandey, A., \& Lodish, H. F. (2002). Context-specific effects of fibulin-5 (DANCE/EVEC) on cell proliferation, motility, and invasion. Fibulin-5 is induced by transforming growth factor-beta and affects protein kinase cascades. J Biol Chem, 277(30), 27367-27377. doi:10.1074/jbc.M200148200

Schleich, J. M., Abdulla, T., Summers, R., \& Houyel, L. (2013). An overview of cardiac morphogenesis. Arch Cardiovasc Dis, 106(11), 612-623. doi:10.1016/j.acvd.2013.07.001

Seay, U., Sedding, D., Krick, S., Hecker, M., Seeger, W., \& Eickelberg, O. (2005). Transforming growth factor-beta-dependent growth inhibition in primary vascular smooth muscle cells is p38-dependent. J Pharmacol Exp Ther, 315(3), 1005-1012. doi:10.1124/jpet.105.091249

Segers, V. F. M., Brutsaert, D. L., \& De Keulenaer, G. W. (2018). Cardiac Remodeling: Endothelial Cells Have More to Say Than Just NO. Front Physiol, 9, 382. doi:10.3389/fphys.2018.00382

Seki, T., Hong, K. H., \& Oh, S. P. (2006). Nonoverlapping expression patterns of ALK1 and ALK5 reveal distinct roles of each receptor in vascular development. Lab Invest, 86(2), 116-129. doi:10.1038/labinvest.3700376

Serluca, F. C. (2008). Development of the proepicardial organ in the zebrafish. Developmental Biology, 315(1), 18-27. doi:10.1016/j.ydbio.2007.10.007

Shao, Y., Li, G., Huang, S., Li, Z., Qiao, B., Chen, D., . . Li, P. (2020). Effects of Extracellular Matrix Softening on Vascular Smooth Muscle Cell Dysfunction. Cardiovasc Toxicol, 20(6), 548556. doi:10.1007/s12012-020-09580-8

Shi, X., Guo, L. W., Seedial, S. M., Si, Y., Wang, B., Takayama, T., .. Kent, K. C. (2014). TGFbeta/Smad3 inhibit vascular smooth muscle cell apoptosis through an autocrine signaling mechanism involving VEGF-A. Cell Death Dis, 5, e1317. doi:10.1038/cddis.2014.282

Sidhwani, P., Boezio, G. L. M., Yang, H., Chi, N. C., Roman, B. L., Stainier, D. Y. R., \& Yelon, D. (2019). Cardiac function modulates endocardial cell dynamics to shape the cardiac outflow tract. bioRxiv, 787358. doi:10.1101/787358

Sidhwani, P., Leerberg, D. M., Boezio, G. L. M., Capasso, T. L., Yang, H., Chi, N. C., ... Yelon, D. (2020). Cardiac function modulates endocardial cell dynamics to shape the cardiac outflow tract. Development, 147(12). doi:10.1242/dev.185900

Sievers, F., Wilm, A., Dineen, D., Gibson, T. J., Karplus, K., Li, W., . . Higgins, D. G. (2011). Fast, scalable generation of high-quality protein multiple sequence alignments using Clustal Omega. Mol Syst Biol, 7, 539. doi:10.1038/msb.2011.75

Silva, A. C., Pereira, C., Fonseca, A., Pinto-do, O. P., \& Nascimento, D. S. (2020). Bearing My Heart: The Role of Extracellular Matrix on Cardiac Development, Homeostasis, and Injury Response. Front Cell Dev Biol, 8, 621644. doi:10.3389/fcell.2020.621644 
Simoes-Costa, M. S., Vasconcelos, M., Sampaio, A. C., Cravo, R. M., Linhares, V. L., Hochgreb, T., .. . Xavier-Neto, J. (2005). The evolutionary origin of cardiac chambers. Developmental Biology, 277(1), 1-15. doi:10.1016/j.ydbio.2004.09.026

Simoes, F. C., \& Riley, P. R. (2018). The ontogeny, activation and function of the epicardium during heart development and regeneration. Development, 145(7). doi:10.1242/dev.155994

Simossis, V. A., \& Heringa, J. (2005). PRALINE: a multiple sequence alignment toolbox that integrates homology-extended and secondary structure information. Nucleic Acids Res, 33(Web Server issue), W289-294. doi:10.1093/nar/gki390

Singh, A., Horsfield, M. A., Bekele, S., Greenwood, J. P., Dawson, D. K., Berry, C., . . McCann, G. P. (2019). Aortic stiffness in aortic stenosis assessed by cardiovascular MRI: a comparison between bicuspid and tricuspid valves. Eur Radiol, 29(5), 2340-2349. doi:10.1007/s00330018-5775-6

Singh, A. R., Sivadas, A., Sabharwal, A., Vellarikal, S. K., Jayarajan, R., Verma, A., ... Sivasubbu, S. (2016). Chamber Specific Gene Expression Landscape of the Zebrafish Heart. PLoS One, 11(1), e0147823. doi:10.1371/journal.pone.0147823

Smith, C. L., Baek, S. T., Sung, C. Y., \& Tallquist, M. D. (2011). Epicardial-derived cell epithelial-tomesenchymal transition and fate specification require PDGF receptor signaling. Circ Res, 108(12), e15-26. doi:10.1161/CIRCRESAHA.110.235531

Smits, A. M., Dronkers, E., \& Goumans, M. J. (2018). The epicardium as a source of multipotent adult cardiac progenitor cells: Their origin, role and fate. Pharmacol Res, 127, 129-140. doi:10.1016/j.phrs.2017.07.020

Son, S., Kang, J. H., Oh, S., Kirschner, M. W., Mitchison, T. J., \& Manalis, S. (2015). Resonant microchannel volume and mass measurements show that suspended cells swell during mitosis. J Cell Biol, 211(4), 757-763. doi:10.1083/jcb.201505058

Sridurongrit, S., Larsson, J., Schwartz, R., Ruiz-Lozano, P., \& Kaartinen, V. (2008). Signaling via the Tgf-beta type I receptor Alk5 in heart development. Developmental Biology, 322(1), 208218. doi:10.1016/j.ydbio.2008.07.038

Srivastava, D. (2006). Making or breaking the heart: from lineage determination to morphogenesis. Cell, 126(6), 1037-1048. doi:10.1016/j.cell.2006.09.003

Stainier, D. Y., \& Fishman, M. C. (1994). The zebrafish as a model system to study cardiovascular development. Trends Cardiovasc Med, 4(5), 207-212. doi:10.1016/1050-1738(94)90036-1

Staudt, D., \& Stainier, D. (2012). Uncovering the molecular and cellular mechanisms of heart development using the zebrafish. Annu Rev Genet, 46, 397-418. doi:10.1146/annurevgenet-110711-155646

Stoller, J. Z., \& Epstein, J. A. (2005). Cardiac neural crest. Semin Cell Dev Biol, 16(6), 704-715. doi:10.1016/j.semcdb.2005.06.004

Stratman, A. N., Pezoa, S. A., Farrelly, O. M., Castranova, D., Dye, L. E., 3rd, Butler, M. G., .. . Weinstein, B. M. (2017). Interactions between mural cells and endothelial cells stabilize the developing zebrafish dorsal aorta. Development, 144(1), 115-127. doi:10.1242/dev.143131

Stroschein, S. L., Wang, W., Zhou, S., Zhou, Q., \& Luo, K. (1999). Negative feedback regulation of TGF-beta signaling by the SnoN oncoprotein. Science, 286(5440), 771-774. doi:10.1126/science.286.5440.771

Stuckmann, I., Evans, S., \& Lassar, A. B. (2003). Erythropoietin and retinoic acid, secreted from the epicardium, are required for cardiac myocyte proliferation. Developmental Biology, 255(2), 334-349. doi:10.1016/s0012-1606(02)00078-7

Sucov, H. M., Dyson, E., Gumeringer, C. L., Price, J., Chien, K. R., \& Evans, R. M. (1994). RXR alpha mutant mice establish a genetic basis for vitamin A signaling in heart morphogenesis. Genes Dev, 8(9), 1007-1018. doi:10.1101/gad.8.9.1007

Sugishita, Y., Watanabe, M., \& Fisher, S. A. (2004). The development of the embryonic outflow tract provides novel insights into cardiac differentiation and remodeling. Trends in Cardiovascular Medicine, 14(6), 235-241. doi:DOI 10.1016/j.tcm.2004.06.004 
Sullivan, K. M., Bissonnette, R., Yanagisawa, H., Hussain, S. N., \& Davis, E. C. (2007). Fibulin-5 functions as an endogenous angiogenesis inhibitor. Lab Invest, 87(8), 818-827. doi:10.1038/labinvest.3700594

Sun, J., Deng, H., Zhou, Z., Xiong, X., \& Gao, L. (2018). Endothelium as a Potential Target for Treatment of Abdominal Aortic Aneurysm. Oxid Med Cell Longev, 2018, 6306542. doi:10.1155/2018/6306542

Sun, L., Lien, C. L., Xu, X., \& Shung, K. K. (2008). In vivo cardiac imaging of adult zebrafish using high frequency ultrasound (45-75 MHz). Ultrasound Med Biol, 34(1), 31-39. doi:10.1016/j.ultrasmedbio.2007.07.002

Suwanabol, P. A., Seedial, S. M., Shi, X., Zhang, F., Yamanouchi, D., Roenneburg, D., . . Kent, K. C. (2012). Transforming growth factor-beta increases vascular smooth muscle cell proliferation through the Smad3 and extracellular signal-regulated kinase mitogenactivated protein kinases pathways. J Vasc Surg, 56(2), 446-454. doi:10.1016/j.jvs.2011.12.038

Sweeney, M., \& Foldes, G. (2018). It Takes Two: Endothelial-Perivascular Cell Cross-Talk in Vascular Development and Disease. Front Cardiovasc Med, 5, 154. doi:10.3389/fcvm.2018.00154

Tabula Muris, C., Overall, c., Logistical, c., Organ, c., processing, Library, p., ... Principal, i. (2018). Single-cell transcriptomics of 20 mouse organs creates a Tabula Muris. Nature, 562(7727), 367-372. doi:10.1038/s41586-018-0590-4

Takahashi, M., Yamagishi, T., Narematsu, M., Kamimura, T., Kai, M., \& Nakajima, Y. (2014). Epicardium is required for sarcomeric maturation and cardiomyocyte growth in the ventricular compact layer mediated by transforming growth factor beta and fibroblast growth factor before the onset of coronary circulation. Congenit Anom (Kyoto), 54(3), 162-171. doi:10.1111/cga.12048

Takeda, N., Hara, H., Fujiwara, T., Kanaya, T., Maemura, S., \& Komuro, I. (2018). TGF-beta Signaling-Related Genes and Thoracic Aortic Aneurysms and Dissections. Int J Mol Sci, 19(7). doi:10.3390/ijms19072125

Tecalco-Cruz, A. C., Rios-Lopez, D. G., Vazquez-Victorio, G., Rosales-Alvarez, R. E., \& Macias-Silva, M. (2018). Transcriptional cofactors Ski and SnoN are major regulators of the TGFbeta/Smad signaling pathway in health and disease. Signal Transduct Target Ther, 3, 15. doi:10.1038/s41392-018-0015-8

Thisse, C., \& Thisse, B. (2008). High-resolution in situ hybridization to whole-mount zebrafish embryos. Nat Protoc, 3(1), 59-69. doi:10.1038/nprot.2007.514

Tirziu, D., Giordano, F. J., \& Simons, M. (2010). Cell communications in the heart. Circulation, 122(9), 928-937. doi:10.1161/CIRCULATIONAHA.108.847731

Tomanek, R. J., Ratajska, A., Kitten, G. T., Yue, X., \& Sandra, A. (1999). Vascular endothelial growth factor expression coincides with coronary vasculogenesis and angiogenesis. Dev Dyn, 215(1), 54-61. doi:10.1002/(SICI)1097-0177(199905)215:1<54::AID-DVDY6>3.0.CO;2-0

Tyser, R. C., Miranda, A. M., Chen, C. M., Davidson, S. M., Srinivas, S., \& Riley, P. R. (2016). Calcium handling precedes cardiac differentiation to initiate the first heartbeat. Elife, 5. doi:10.7554/eLife.17113

Uribe, V., Ramadass, R., Dogra, D., Rasouli, S. J., Gunawan, F., Nakajima, H., . . Stainier, D. Y. R. (2018). In vivo analysis of cardiomyocyte proliferation during trabeculation. Development, 145(14). doi:10.1242/dev.164194

Valiente-Alandi, I., Schafer, A. E., \& Blaxall, B. C. (2016). Extracellular matrix-mediated cellular communication in the heart. J Mol Cell Cardiol, 91, 228-237. doi:10.1016/j.yjmcc.2016.01.011

van Boxtel, A. L., Chesebro, J. E., Heliot, C., Ramel, M. C., Stone, R. K., \& Hill, C. S. (2015). A Temporal Window for Signal Activation Dictates the Dimensions of a Nodal Signaling Domain. Dev Cell, 35(2), 175-185. doi:10.1016/j.devcel.2015.09.014 
van de Pol, V., Kurakula, K., DeRuiter, M. C., \& Goumans, M. J. (2017). Thoracic Aortic Aneurysm Development in Patients with Bicuspid Aortic Valve: What Is the Role of Endothelial Cells? Front Physiol, 8, 938. doi:10.3389/fphys.2017.00938

Varshney, G. K., Pei, W., LaFave, M. C., Idol, J., Xu, L., Gallardo, V., .. Burgess, S. M. (2015). Highthroughput gene targeting and phenotyping in zebrafish using CRISPR/Cas9. Genome Res, 25(7), 1030-1042. doi:10.1101/gr.186379.114

Vega-Hernandez, M., Kovacs, A., De Langhe, S., \& Ornitz, D. M. (2011). FGF10/FGFR2b signaling is essential for cardiac fibroblast development and growth of the myocardium. Development, 138(15), 3331-3340. doi:10.1242/dev.064410

Villars, A., \& Levayer, R. (2020). Cell Extrusion: Crowd Pushing and Sticky Neighbours. Curr Biol, 30(4), R168-R171. doi:10.1016/j.cub.2019.12.033

Villefranc, J. A., Amigo, J., \& Lawson, N. D. (2007). Gateway compatible vectors for analysis of gene function in the zebrafish. Dev Dyn, 236(11), 3077-3087. doi:10.1002/dvdy.21354

Waldo, K. L., Hutson, M. R., Stadt, H. A., Zdanowicz, M., Zdanowicz, J., \& Kirby, M. L. (2005). Cardiac neural crest is necessary for normal addition of the myocardiurn to the arterial pole from the secondary heart field. Developmental Biology, 281(1), 66-77. doi:10.1016/j.ydbio.2005.02.011

Waldo, K. L., Hutson, M. R., Ward, C. C., Zdanowicz, M., Stadt, H. A., Kumiski, D., . . Kirby, M. L. (2005). Secondary heart field contributes myocardium and smooth muscle to the arterial pole of the developing heart. Developmental Biology, 281(1), 78-90. doi:10.1016/j.ydbio.2005.02.012

Wang, G., Jacquet, L., Karamariti, E., \& Xu, Q. (2015). Origin and differentiation of vascular smooth muscle cells. J Physiol, 593(14), 3013-3030. doi:10.1113/JP270033

Wang, J., Panakova, D., Kikuchi, K., Holdway, J. E., Gemberling, M., Burris, J. S., . . Poss, K. D. (2011). The regenerative capacity of zebrafish reverses cardiac failure caused by genetic cardiomyocyte depletion. Development, 138(16), 3421-3430. doi:10.1242/dev.068601

Wang, J., Cao, J., Dickson, A. L., \& Poss, K. D. (2015). Epicardial regeneration is guided by cardiac outflow tract and Hedgehog signalling. Nature, 522(7555), 226-230. doi:10.1038/nature14325

Wang, L., Zhang, J., Fu, W., Guo, D., Jiang, J., \& Wang, Y. (2012). Association of smooth muscle cell phenotypes with extracellular matrix disorders in thoracic aortic dissection. J Vasc Surg, 56(6), 1698-1709, 1709 e1691. doi:10.1016/j.jvs.2012.05.084

Wang, Y., Ait-Oufella, H., Herbin, O., Bonnin, P., Ramkhelawon, B., Taleb, S., . . Mallat, Z. (2010). TGF-beta activity protects against inflammatory aortic aneurysm progression and complications in angiotensin II-infused mice. J Clin Invest, 120(2), 422-432. doi:10.1172/JCl38136

Wang, Y., Kaiser, M. S., Larson, J. D., Nasevicius, A., Clark, K. J., Wadman, S. A., . . Essner, J. J. (2010). Moesin 1 and Ve-cadherin are required in endothelial cells during in vivo tubulogenesis. Development, 137(18), 3119-3128. doi:10.1242/dev.048785

Weinberger, M., Simoes, F. C., Patient, R., Sauka-Spengler, T., \& Riley, P. R. (2020). Functional Heterogeneity within the Developing Zebrafish Epicardium. Dev Cell, 52(5), 574-590 e576. doi:10.1016/j.devcel.2020.01.023

Wharton, K., \& Derynck, R. (2009). TGFbeta family signaling: novel insights in development and disease. Development, 136(22), 3691-3697. doi:10.1242/dev.040584

Whitesell, T. R., Kennedy, R. M., Carter, A. D., Rollins, E. L., Georgijevic, S., Santoro, M. M., \& Childs, S. J. (2014). An alpha-smooth muscle actin (acta2/alphasma) zebrafish transgenic line marking vascular mural cells and visceral smooth muscle cells. PLoS One, 9(3), e90590. doi:10.1371/journal.pone.0090590

Williamson, M. R., Shuttleworth, A., Canfield, A. E., Black, R. A., \& Kielty, C. M. (2007). The role of endothelial cell attachment to elastic fibre molecules in the enhancement of monolayer formation and retention, and the inhibition of smooth muscle cell recruitment. Biomaterials, 28(35), 5307-5318. doi:10.1016/j.biomaterials.2007.08.019 
Wong, S. H., Hamel, L., Chevalier, S., \& Philip, A. (2000). Endoglin expression on human microvascular endothelial cells association with betaglycan and formation of higher order complexes with TGF-beta signalling receptors. Eur J Biochem, 267(17), 5550-5560. doi:10.1046/j.1432-1327.2000.01621.x

Wu, B., Zhang, Z., Lui, W., Chen, X., Wang, Y., Chamberlain, A. A., . . Zhou, B. (2012). Endocardial cells form the coronary arteries by angiogenesis through myocardial-endocardial VEGF signaling. Cell, 151(5), 1083-1096. doi:10.1016/j.cell.2012.10.023

Wu, M. Y., \& Hill, C. S. (2009). Tgf-beta superfamily signaling in embryonic development and homeostasis. Dev Cell, 16(3), 329-343. doi:10.1016/j.devcel.2009.02.012

Yan, X., Xiong, X., \& Chen, Y. G. (2018). Feedback regulation of TGF-beta signaling. Acta Biochim Biophys Sin (Shanghai), 50(1), 37-50. doi:10.1093/abbs/gmx129

Yanagisawa, H., Schluterman, M. K., \& Brekken, R. A. (2009). Fibulin-5, an integrin-binding matricellular protein: its function in development and disease. J Cell Commun Signal, 3(34), 337-347. doi:10.1007/s12079-009-0065-3

Yang, J. T., Rayburn, H., \& Hynes, R. O. (1995). Cell adhesion events mediated by alpha 4 integrins are essential in placental and cardiac development. Development, 121(2), 549-560.

Yang, P., Schmit, B. M., Fu, C., DeSart, K., Oh, S. P., Berceli, S. A., \& Jiang, Z. (2016). Smooth muscle cell-specific Tgfbr1 deficiency promotes aortic aneurysm formation by stimulating multiple signaling events. Sci Rep, 6, 35444. doi:10.1038/srep35444

Yu, G., Wang, L. G., Han, Y., \& He, Q. Y. (2012). clusterProfiler: an R package for comparing biological themes among gene clusters. OMICS, 16(5), 284-287. doi:10.1089/omi.2011.0118

Zhang, P., Hou, S., Chen, J., Zhang, J., Lin, F., Ju, R., .. Yang, X. (2016). Smad4 Deficiency in Smooth Muscle Cells Initiates the Formation of Aortic Aneurysm. Circ Res, 118(3), 388399. doi:10.1161/CIRCRESAHA.115.308040

Zhou, Y., Cashman, T. J., Nevis, K. R., Obregon, P., Carney, S. A., Liu, Y., . . Burns, C. G. (2011). Latent TGF-beta binding protein 3 identifies a second heart field in zebrafish. Nature, 474(7353), 645-648. doi:10.1038/nature10094

Zlotek-Zlotkiewicz, E., Monnier, S., Cappello, G., Le Berre, M., \& Piel, M. (2015). Optical volume and mass measurements show that mammalian cells swell during mitosis. $J$ Cell Biol, 211(4), 765-774. doi:10.1083/jcb.201505056

Zygmunt, T., Gay, C. M., Blondelle, J., Singh, M. K., Flaherty, K. M., Means, P. C., . . TorresVazquez, J. (2011). Semaphorin-PlexinD1 signaling limits angiogenic potential via the VEGF decoy receptor sFlt1. Dev Cell, 21(2), 301-314. doi:10.1016/j.devcel.2011.06.033 


\section{Acknowledgments}

First and most importantly, I would like to thank my supervisor Prof. Didier Stainier. Didier shaped my approach to science in several ways. I am extremely grateful for the freedom he gave me and the trust he showed innumerable times. Didier left me free to develop my ideas and shape my projects, but he was always available whenever I needed guidance. He taught me how to manage a project, how far to push it, and when to say "it's enough". I had the opportunity to attend several conferences, getting feedback and establishing connections, and to mentor several students, which was one of the most rewarding experiences of my $\mathrm{PhD}$. I thank him for his incredible support during challenging and demotivating times but also his shared enthusiasm in very successful moments. Didier also took care of pushing me out of the nest and outside of my comfort zone when it was time for me to start exploring new options for my future career, and I am immensely grateful for that. I hope I can continue to benefit from his mentorship from far away.

I also would like to thank Prof. Dr. Amparo Acker-Palmer for her support and feedback over the years as my thesis advisory committee member, and for reviewing this thesis.

Also a part of my thesis advisory committee, Prof. Christian Helker has been a great supervisor and the first person who introduced me to the lab. With time, he has also become a good friend. As a supervisor, I fully owe him my ability to be efficient, multitask, prioritize... and walk fast. He taught me not to lose sight of the big picture, to be concrete, focus, and put the experiments into perspective. One of the most inspiring things I learned from him as a supervisor (and a person) was his openness to feedback and the way he incorporated them in his mentoring style.

Anabela, Felix, and Rashmi have been my unofficial but invaluable mentors, as well as unique friends. Each in a different way, they have been my source of motivation, encouragement, my example of integrity and hard work. Anabela has been my biggest cheerleader during the $\mathrm{PhD}$, and I firmly believe that everybody needs one to succeed. I owe her a lot of enthusiasm and self-confidence in challenging times. Personally, Rashmi was my mom outside of home, the first person who shared with me the house and much more. Professionally, I thank her especially for keeping me focused, not sparing criticisms when needed, and inspiring me -with her example- never to settle. Felix's curiosity and enthusiasm are contagious and his scientific integrity is admirable. I am extremely grateful to him for being always available to discuss and brainstorm, to provide excellent ideas, and for always valuing my feedback.

The discussions with Paolo, Michelle, Rubèn, and Carol have been irreplaceable in shaping my scientific personality. I would like to thank (in random order) Kenny Mattonet, Sebastian Gauvrit, Andrea Rossi, Zacharias Kontarakis, Jane Jiang, Sam Capon, Thomas Juan, Anna Sokol, Claudia Gerri, Michele Marass, Arica Beisaw, Chi-Chung Wu, Hyun-Taek Kim, Sophie Ramas, Vahan Serobyan, Sven Reischauer, Veronica Uribe Sokolov, Javad Rasouli, Deepika Dogra, Pinelopi Goumenaki, Mridula Balakrishnan, Jordan Welker, Srinath Ramkumar, Jialing Qi, João Cardeira, Vanesa Jimenez, and Radhan Ramadass for the intellectual discussions, support and a lot of fun. I have to thank all the people who joined later over the years, for contributing to keeping the lab a fun, friendly, and collaborative environment as I have seen 
few. I am also grateful to the "new" PhD students (Agatha, Pinelopi, Shengnan, Marga, Yanli, Armaan, Savita, Sapna, Preethi), for giving us the responsibility of being "senior" and for trusting us. I hope we have met your expectations.

Special thanks go to Felix, Michelle, and Matteo for reading and commenting on my thesis, and to Conni and Kenny for helping me with a fantastic German translation of the summaries.

Additionally, I am thankful to all the collaborators I worked with over the years both in the lab and outside, such as Prof. Valerie Wittamer, Prof. Deborah Yelon, and Dr. Pragya Sidwhani, Thanks to them, I learned how teamwork should be. Working with them, I expanded my skills to topics unrelated to my $\mathrm{PhD}$ work. I also learned to share responsibilities, delegate, and contribute to a bigger picture. I also would like to thank Stefan Günther, Ann Atzberger, Kikhi Khrievono, Janett Piesker and Astrid Wietelmann for their expert contribution to my projects.

I have learned immensely from the students I had the luck and the honor to mentor, both officially and unofficially. Conni, Zephie, Sertap, Marlene, and Helen were a continuous source of motivation, enthusiasm, and an example of dedication. They pushed me to improve every day to be the scientist and the person they deserved to be mentored by.

In the Stainier lab, I was lucky to work with a fantastic team, which made our work much easier. I would like to thank especially Sharon, who has gone further than what she was supposed to do to help me many many times. I am very grateful to Hans-Martin, Nana, Marianne, Petra, Sarah, Beate, Carmen K, Carmen B., Kikhi, Rebecca, Monika. I would like to thank immensely Sabine and Simon, who made our fish work incredibly easier. I also thank Rita Retzloff, Nouha Ritschel, Jennifer Behm, Monika Mueller-Boche, Martin Laszczyk, Michelle Pauels and the entire zebrafish facility for their help.

I would like to thank Bilge Reischauer and the PhD Committee(s) (2017-2019) for sharing a fantastic experience, in which I learned invaluable skills and had a lot of fun.

Being for the first time outside of home, I was fortunate to find here a second family. Ale, Claudia, Srinivas, Teja, Gabriel, Mohamed, and Hadil have been vital friends throughout this journey. If now it feels like leaving home again, I owe it specifically to them. I would spend pages thanking each of you separately, but I hope -by now- you know everything already.

Ale and Gabriel have been the BEST office-mates anyone can ask for: replacing them will be impossible. Simon and Paolo helped to bring some Italy to Germany making me feel more at home and sharing a lot of fun. Julia has been a fantastic friend and a partner in many experiences from the very beginning. She helped me navigating German traditions, bureaucracy, and -more in general- German life. Each in their own ways, they have been with me, supported me, made fun of me, helped me, scolded me when needed. Scientifically and personally, from them, I learned things I am confident I will bring forever with me. In all the challenges of a $\mathrm{PhD}$, I am lucky to say that there was not a single moment in which I felt alone. 
Dr. Monica Beltrame was the first person who introduced me to my scientific career being my Master's supervisor. She has always been a fantastic mentor and taught me what passion, honesty, dedication, and patience mean. During the $\mathrm{PhD}$, she has continued to stand by my side and give me advice, and I am really grateful to still have her support.

My long-time friends have been a unique support from far away. Carlotta, Riccardo, Fede, Stefania, Silvia, Isabella, Chiara, Elettra, Anna, Annalisa, Luca, Fabio, Maita have never stopped being with me. With our different lives and backgrounds, they helped me to put things into perspective every time we talked. They have cheered with me, tried to understand what I was doing, and even learned to love zebrafish through my stories. Thank you for keeping me always connected to what really matters in life.

Laura and Nadia have been indispensable during these years. We explored Europe together to recharge the batteries when needed, we had serious and less serious conversations, shared $\mathrm{PhD}$ experiences and food recipes, cheered for each other, and shared the inevitable frustration sometimes. Thanks to all of this, I never felt the distance between us for a second. Thank you for always having my back.

Thanking Matteo here will never do him justice. I can just say that I have been fortunate to spend the last 11 years of my life with the best person and the best scientist I have ever met. All the difficult things, next to him, felt light and easy. What I thank him the most for was convincing me that I could have done everything even alone. He concretely showed me the meaning of the word “empowered". I couldn't have been luckier than this.

This achievement would not have been possible without the support of my family. Leaving home for the first time was not easy, but their unconditional love made it feasible and rewarding. I wish to thank all of them for taking the time to listen and ask questions about my work, for trying to understand the difficulties, and for cheering on my success. Making them proud was, is, and will be one of my biggest driving forces. Lastly, I would like to thank my mom, to whom I dedicate my thesis (once again). She has been my pillar, anchor, and example in every moment. The relationship we have fills my heart with joy and pride every day. Grazie, mamma.

Questo risultato non sarebbe stato possibile senza il sostegno della mia famiglia. Lasciare casa per la prima volta non è stato facile, ma il loro amore incondizionato lo ha reso fattibile e anche gratificante. Desidero ringraziarli per aver dedicato del tempo ad ascoltarmi e fare domande sul mio lavoro, per aver cercato di comprendere le sfide e le difficoltà, e per aver gioito per i miei successi. Renderli orgogliosi è stato, è e sarà uno dei tanti motori della mia carriera. Infine, vorrei ringraziare la mia mamma, a cui dedico la mia tesi (ancora una volta). È stata il mio pilastro, àncora ed esempio in ogni momento. La relazione che abbiamo mi riempie il cuore di orgoglio e di gioia ogni giorno. Grazie, mamma. 


\section{Curriculum Vitae}

\section{Giulia Ludovica Maddalena Boezio}

Address

Nationality

Haupstraße 7, 61231, Bad Nauheim, Germany

Date of birth

Italian

Place of birth

$27 / 08 / 1991$

E-mail

Segrate (Milan), Italy

ORCiD

Giulia.Boezio@mpi-bn.mpg.de; giulia.boezio@gmail.com

0000-0002-7776-7985

\section{EDUCATION AND TRAINING}

- October 2016-present

PhD in Developmental Genetics

IMPRS-HLR PhD school, Max Planck Institute for Heart and Lung Research (Germany)

- September 2013-December 2015

MSc in Molecular Biology of the Cell

University of Milan (Italy); 110/110 summa cum laude

- September 2010- July 2013

BSc in Biological Sciences

University of Milan (Italy); 110/110 summa cum laude

- September 2005-July 2010

High School for classical studies

Liceo Berchet, Milan (Italy); 100/100

\section{RESEARCH EXPERIENCE}

- October 2016- May 2021

PhD at the Developmental Genetics Department

Max Planck Institute for Heart and Lung Research, Germany

Supervisors: Prof. Didier Stainier, Prof. Christian Helker

Main project: "Endothelial Alke5 instructs smooth muscle cell development in the zebrafish cardiac outflow tract"

- January 2016 - August 2016

Post-MSc fellowship

University of Milan, Italy

Supervisor: Dr. Monica Beltrame

Title of the project: "Sox13 at the crossroad of signaling pathways regulating angiogenesis" 
- September 2014-December 2015

MSc thesis internship

University of Milan, Italy

Supervisor: Dr. Monica Beltrame

Title of the thesis: "Sox13 role in angiogenesis: phenotype and molecular mechanisms in gene

knockdown conditions in zebrafish"

\section{PUBLICATIONS}

- Inpreparation: Boezio GLM, Gollin J, Fukuda N, Gunawan F, Stainier DYR. The epicardium promotes cardiomyocyte growth and volume increase during heart development

1. Boezio GLM, Bensimon-Brito A, Guenther S, Piesker J, Helker CSM, Stainier DYR (2020) Endothelial TGF- $\beta$ signaling instructs smooth muscle cell development in the cardiac outflow tract (eLife, doi: 10.7554/eLife.57603)

2. Bensimon-Brito A*, Boezio GLM*, Cardeira J, Wietelmann A, Helker CSM, Ramadass R, Piesker J, Nauerth A, Müller C, Stainier DYR (2020). Integration of multiple imaging platforms to uncover cardiac defects in adult zebrafish (under revision, Cardiovascular Research, bioRxiv doi:10.1101/2020.05.21.108548) *, equal contribution

3. Sidhwani P, Leerberg DM, Boezio GLM, Capasso TL, Yang H, Chi NC, Roman BL, Stainier DYR, Yelon D (2020). Cardiac function modulates endocardial cell dynamics to shape the cardiac outflow tract. Development, doi: 10.1242/dev.185900

4. Bensimon-Brito A, Ramkumar S*, Boezio GLM*, Guenther S, Kuenne C, Sanchez-Iranzo H, Iloska D, Piesker J, Pullamsetti S, Mercader N, Beis D, Stainier DYR (2020). TGF-B signaling promotes tissue formation during cardiac valve regeneration in adult zebrafish.

Dev Cell, doi:10.1016/j.devcel.2019.10.027*, equal contribution

5. Mullapudi ST, Boezio GLM, Rossi A, Marass M, Matsuoka RL, Matsuda H, Helker CSM, Yang YHC, Stainier DYR (2019). Disruption of pancreatic vasculature affects islet architecture \& function. Development, doi: 10.1242/dev.173674

6. El-Brolosy MA, Kontarakis Z, Rossi A, Kuenne C, Günther B, Fukuda N, Kikhi K, Boezio GLM, Takacs CM, Lai S, Fukuda R, Gerri C, Giraldez AJ \&. Stainier DYR (2019) Genetic Compensation Triggered by Mutant mRNA Degradation. Nature, 568,193197 doi:10.1038/s41586-019-1064-z

7. Mullapudi ST, Helker CS, Boezio GL, Maischein HM, Sokol AM, Guenther S, Matsuda H, Kubicek S, Graumann J, Yang YHC, \& Stainier DY (2018). Screening for insulinindependent pathways that modulate glucose homeostasis identifies androgen receptor antagonists. eLife, 7, e42209. doi:10.7554/eLife.42209

\section{ACCOMPLISHMENTS/AWARDS}

- Winner of Best Poster Award at European Developmental Biology Congress EDBC (Alicante, Spain, 2019)

- Winner of travel award "Anita Roberts" at the "The TGF-B Superfamily Conference: Signaling 
in Development and Disease" (West Palm Beach, US, 2019) (only one Travel award available for PhD Students)

- Winner of Best Poster award (International Max Planck Research School Retreat, 2019)

- Winner of Best Talk award (International Max Planck Research School Retreat, 2018)

- Ranked top 1\% in the MSc for Molecular Biology of the Cell (University of Milan, 2014-2015)

- Scholarship for excellent academic achievements (University of Milan, 2014-2015)

- BSc, MSc summa cum laude

\section{CONFERENCES \& MEETINGS}

- IRIBHM interdisciplinary seminars (ULB, Belgium/Online, 2020/2021) Invited talk

- Society for Developmental Biology $79^{\text {th }}$ Annual Meeting (Online, 2020) Poster

- Virtual Fallot Club (University Aix-Marseille/Online, 2020) Talk

- CRC1366: Vascular Mini-Symposium (Bad Nauheim 2020, canceled Covid-19) Invited talk

- European Developmental Biology Conference (Alicante, Spain, 2019), Flash talk and poster

- The TGF-B Superfamily Conference: Signaling in Development and Disease (West Palm Beach, Florida, US, 2019), Talk selected from abstracts

- IMPRS 12+ evaluation (Bad Nauheim 2019), Talk

- Cardio Pulmonary Institute Retreat (Bad Nauheim 2019), Poster

- Membrane Plasticity in Tissue Development and Remodeling (Marburg 2019), Talk

- IMPRS PhD School Retreat (Weilburg 2018), Talk

- $20^{\text {th }}$ International Vascular Biology Meeting (Helsinki, Finland, 2018), Poster

- $3^{\text {rd }}$ Fish Regional Meeting (Heidelberg 2018), Poster

- MPI-HLR Institute Retreat (Bad Marienberg 2018), Talk

- Frontiers in Vascular Biology Retreat (Berlin 2018)

- IMPRS PhD School Retreat (Schloss Ringberg 2017), Poster

- Vascular Differentiation and Remodeling (Heidelberg 2017)

- MPI-HLR Institute Retreat (Schloss Ringberg 2016)

\section{CERTIFIED TRAINING AND COURSE}

Image Processing (Fiji ImageJ, Imaris)

Confocal Microscopy

Wilson workshop for RNAseq analysis

MRI, microCT

Electron Microscopy
Statistics for biomedical research

FELASA - Fish Handling

FELASA - Mouse Handling and Surgery

Communicating Science to the Public

Scientific Writing

Oral and Poster Presentation

\section{PUBLIC OUTREACH ACTIVITIES}

- Contributor to Skype a Scientist (https://www.skypeascientist.com/ ) 10/2020-present

- Contributor to "Letters to a Pre-Scientist" (https://www.prescientist.org/about-us/) 07/2020-present

- Contributor at "preLights" (https://prelights.biologists.com/ ) 03/2019-present

- Part of "OpenScience" group of MPG PhDnet, 11/2018-11/2020

- Contributor to Offspring podcast (MPG PhDnet), 2020 (1 podcast episode) 
- Co-founder of a scientific Instagram page (@sci_comm) to raise scientific awareness and contribute to public outreach for a non-scientist young audience

- External PhD representative MPI-HLR, 05/2018 - 06/2019

- Member of $\mathbf{P h D}$ committee involved in the organization of $\mathrm{PhD}$ retreats with international keynote speakers, 10/2017-10/2019

\section{TEACHING}

- Supervision of two Master students (5 weeks, 2018; 4 months 2019-2020)

- Supervision of three Bachelor students (3 months, 2017; 2 months, 2019; 1 month, 2020) 